\title{
Development of a Cost-effective High-fidelity Type-specific Flight Simulator with Emphasis on Flight Modelling
}

\author{
by \\ Suzanne Swaine, B.Eng. \\ Carleton University \\ A thesis submitted to \\ the Faculty of Graduate Studies and Research \\ in partial fulfillment of \\ the requirements for the degree of \\ Masters of Applied Science \\ Ottawa-Carleton Institute for \\ Mechanical and Aerospace Engineering \\ Department of \\ Mechanical and Aerospace Engineering \\ Carleton University \\ Ottawa, Ontario \\ April 3, 2014 \\ (C) Copyright \\ 2014 - Suzanne Swaine
}




\section{Abstract}

This project was an investigation into the feasibility of developing a cost-effective highfidelity type-specific small aircraft simulator for use as part of an ab-initio flight training program. The development of the KatanaSim, a Diamond DA20-A1 Katana flight simulator built with commercial off-the-shelf components and original aircraft parts wherever possible, proved the feasibility of the endeavour. Particularly successful was the high level of physical fidelity achieved by the use of original aircraft parts, most notably a Katana fuselage. The development cost of the KatanaSim was significantly less than the six and seven figure costs usually associated with high-fidelity flight simulators.

In order to acquire the data necessary for flight model evaluation and tuning, a minimally-intrusive flight testing methodology for small aircraft was developed. A compact instrumentation package was designed and tested, and a flight permit from Transport Canada was obtained. Two flight tests in a DA20-A1 Katana were completed and several hours worth of flight testing data was acquired.

X-Plane, by Laminar Research, was chosen as the core simulator software and Plane Maker, a part of the same package, was used to develop the flight model. Aircraft model parameters were obtained from published data sources, and empirical measurements and observations. The resulting flight model required additional tuning to meet the desired performance specifications. A curve-fitting genetic algorithm was developed to automate the tuning process and was validated using a variety of dynamic models. This genetic algorithm was proven to be capable of tuning the stability derivatives of an aircraft given simulated flight performance data. In future, the genetic algorithm can be used with a blade element theory mathematical model to complete the tuning of the KatanaSim flight model using the acquired flight test data. 


\section{Acknowledgements}

I'd like to acknowledge the faculty, staff, and students at Carleton who made this ambitious project possible; our industry sponsors, particularly the OAS maintenance team for their support and guidance throughout the project; and Ashraf Othman, for his generous donation of time and expertise that made flight testing possible. My biggest thanks goes to Team KatanaSim, for sticking with this project through a seemingly endless set of challenges. 
To my parents, the pilot and the scientist, who inspired my long and winding journey and made me who I am today.

To Katie, Drew, Mike, Jeremy, Brent, and Andrew, who were always ready with words of encouragement, advice, and love.

But most of all to Rob, my greatest teacher, who went above and beyond every step of the way, and who got me here despite my best efforts at being impossible. 


\section{Contents}

Abstract

$\begin{array}{ll}\text { Acknowledgements } & \text { iii }\end{array}$

Dedication $\quad$ iv

Contents

List of Figures $\quad$ x

List of Tables $\quad$ XV

List of Symbols $\quad$ xvi

1 Introduction $\quad 1$

1.1 A Brief History of Flight Simulators . . . . . . . . . . . . . . . 1

1.2 Simulation Fidelity and Certification . . . . . . . . . . . . . 5

1.3 Benefits of Flight Simulator Training . . . . . . . . . . . . . . . . 7

1.3.1 Simulator Use During Ab-initio Training . . . . . . . . . . . . 7

1.3.2 Factors Affecting Transfer of Training . . . . . . . . . . . . . . 8

1.4 Small Aircraft Simulator Research . . . . . . . . . . . . . . . . . . . 10

1.5 The KatanaSim Project . . . . . . . . . . . . . . . . . 12 
1.6 Thesis Overview . . . . . . . . . . . . . . . . . . . . . . . . . . . . . . . . . 13

1.6.1 KatanaSim Hardware . . . . . . . . . . . . . . . . . . . . 13

1.6.2 Flight Testing . . . . . . . . . . . . . . . . . . . . . . . 14

1.6.3 Flight Model . . . . . . . . . . . . . . . . . . . . . . . . . . . . . 14

1.6.4 Automated Flight Model Tuning . . . . . . . . . . . . . . 15

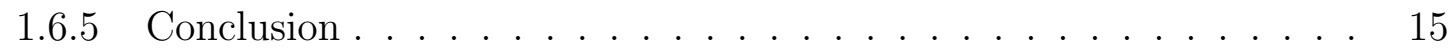

2 KatanaSim Hardware $\quad 16$

2.1 Overview . . . . . . . . . . . . . . . . . . . . . . . . . 16

2.2 Body and Support Structure . . . . . . . . . . . . . . . . . . 17

2.3 Flight Controls . . . . . . . . . . . . . . . . . . . . . . . . 18

2.4 Instrument Panel . . . . . . . . . . . . . . . . . . . . . . . . . . . . 22

2.4 .1 Flight Gauges . . . . . . . . . . . . . . . . . . 24

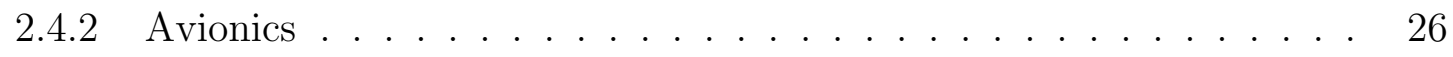

2.4 .3 Engine Gauges $\ldots \ldots \ldots \ldots \ldots$

2.4.4 Switches and Circuit Breakers . . . . . . . . . . . . . . . . . 29

2.4.5 Completed Instrument Panel . . . . . . . . . . . . . . . 30

2.5 Audio Cueing . . . . . . . . . . . . . . . . . . . . . . . . . . . . . 32

2.6 Visual Cueing . . . . . . . . . . . . . . . . . . . . . . . . . . . . . . . . 32

2.7 Brakes . . . . . . . . . . . . . . . . . . . . . . 34

2.8 Haptic Feedback . . . . . . . . . . . . . . . . . . . . . . . . . . . . . . . . 39

2.8 .1 Requirements . . . . . . . . . . . . . . . . 40

2.8 .2 Design and Installation . . . . . . . . . . . . . . 40

2.9 Electrical Components . . . . . . . . . . . . . . . . . . . . . . 43

2.9.1 Instrument Panel Components . . . . . . . . . . . . . . . . 44

2.9.2 Centre Console, Propeller Pitch, and Throttle Levers . . . . . . . 44

2.10 Computer Architecture . . . . . . . . . . . . . . . . . . 45 
2.11 Motion Cueing . . . . . . . . . . . . . . . . . 46

2.12 Software Selection . . . . . . . . . . . . . . . . . . . . . 48

2.13 Budget . . . . . . . . . . . . . . . . . . . . . . . . . . . . 49

2.14 Discussion . . . . . . . . . . . . . . . . . . . . . . 50

3 Flight Testing $\quad 53$

3.1 Motivation for Flight Testing . . . . . . . . . . . . . . . 54

3.2 Test Plan Development . . . . . . . . . . . . . . . . . . . 56

3.3 Equipment and Installation $\ldots \ldots \ldots \ldots \ldots$

3.3 .1 Equipment Mount . . . . . . . . . . . . . . . . 57

3.3.2 Data Recording Devices . . . . . . . . . . . . . . . 58

3.3 .3 Control Inputs . . . . . . . . . . . . . . . . . . . . . . 60

3.3 .4 Sensors . . . . . . . . . . . . . . . . . . . . . . . 62

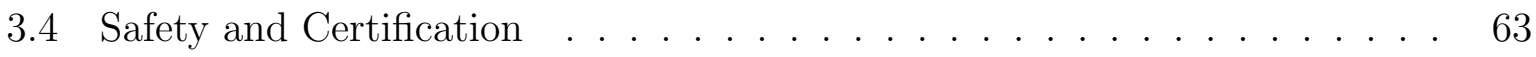

3.4 .1 Equipment Design . . . . . . . . . . . . . . . . . 64

3.4.2 Flight Permit Acquisition . . . . . . . . . . . . . 66

3.5 Equipment Testing . . . . . . . . . . . . . . . . . . . . . . . 67

3.6 Flight Test . . . . . . . . . . . . . . . . . . . . . . . . 68

3.7 Data Processing and Analysis . . . . . . . . . . . . . . . 70

3.7.1 Control Column and Pedal Inputs . . . . . . . . . . . . . 71

3.7.2 Issues Encountered During Post-processing . . . . . . . . . . 78

3.8 Discussion . . . . . . . . . . . . . . . . . . . . . . . . 80

4 Flight Model $\quad 82$

4.1 Plane Maker . . . . . . . . . . . . . . . . . . . . . . . . . . . . . . 82

4.1.1 Blade Element Theory . . . . . . . . . . . . . . . . . 84

4.2 A Katana Flight Model . . . . . . . . . . . . . . . . . . . . . . . . 86 
4.2.1 Katana Specification Research . . . . . . . . . . . . . . . 87

$4.2 .2 \quad$ Building the Flight Model . . . . . . . . . . . . . . . . . . 89

4.3 Model Evaluation . . . . . . . . . . . . . . . . . . . . . . . . . . . . . . . . 91

4.3.1 Climb with Take-off Configuration _. . . . . . . . . . . 92

$4.3 .2 \quad$ Straight and Level Flight . . . . . . . . . . . . . . . . . . . 94

$4.3 .3 \quad$ Stalls . . . . . . . . . . . . . . . . . . . . . . . . . 97

4.4 Discussion . . . . . . . . . . . . . . . . . . . . . . . . . . . 98

5 Automated Flight Model Tuning $\quad 100$

5.1 Proven Flight Modelling Methods . . . . . . . . . . . . . . . . . . 100

5.1 .1 Output Error Method . . . . . . . . . . . . . . . . . . . 101

$5.1 .2 \quad$ Kalman Filters . . . . . . . . . . . . . . . . . . . . . . . 102

5.1 .3 Neural Networks . . . . . . . . . . . . . . . . . . . . . 103

5.1 .4 Genetic Algorithms . . . . . . . . . . . . . . . . . . . . 104

5.2 Use of Stability Derivatives in Flight Dynamics Modelling . . . . . . . 105

5.2.1 Derivation of Longitudinal Rigid Body Equations of Motion . . . . 106

5.3 Development of a Genetic Algorithm for Automated Flight Model Tuning . 115

5.3 .1 Modelling Evolution . . . . . . . . . . . . 116

5.3.2 Development of the Genetic Algorithm . . . . . . . . . . . 120

5.4 Tuning Stability Derivatives Using the Developed Genetic Algorithm . . . 124

5.4.1 Tuning of Two-dimensional Stability Derivatives . . . . . . . . . 126

5.4.2 Tuning of Three-dimensional Stability Derivatives . . . . . . . 127

5.5 Discussion . . . . . . . . . . . . . . . . . . . . . . . 130

$\begin{array}{lll}6 & \text { Conclusion } & 135\end{array}$

6.1 Contributions . . . . . . . . . . . . . . . . 135

6.2 KatanaSim Assessment . . . . . . . . . . . . . . . . . . . 137 
6.3 Future Work . . . . . . . . . . . . . . . . . . . . . . . . . . . . . . . . 139

$\begin{array}{ll}\text { References } & 141\end{array}$

$\begin{array}{ll}\text { Appendices } & 145\end{array}$

A Quotation from Servos and Simulation, Inc. for a Haptic Feedback System

$\begin{array}{ll}\text { B Battery Fire Procedure } & 148\end{array}$

$\begin{array}{lr}\text { C Flight Permit } & 149\end{array}$

D Raw Data Sheets from Flight Tests 152

$\begin{array}{ll}\text { E Equations for Control Input Data Processing } & 171\end{array}$

$\begin{array}{ll}\text { F Sensitivity Study Results } & 173\end{array}$ 


\section{List of Figures}

1.1 Mechtronix full-flight simulator $\mathrm{X}^{T \mathrm{TM}}$ 737-800 exterior and interior . . . . 5

1.2 A DA20-A1 Diamond Katana .................... 13

2.1 Katana fuselage, stripped bare and cut down to size . . . . . . . . . . 17

2.2 KatanaSim fuselage mount . . . . . . . . . . . . . . . . . . . . . . 18

2.3 Diagram of the rudder assembly in a Katana aircraft from the Aircraft Maintenance Manual . . . . . . . . . . . . . . . . . . . . . . . . 19

2.4 KatanaSim profile showing placement of the forward control bulkhead, Bbulkhead, and baggage compartment .............. 20

2.5 Elevator assembly in a Katana aircraft . . . . . . . . . . . . . . . . . . . 21

2.6 Aileron assembly in a Katana aircraft . . . . . . . . . . . . . . . . . . . . . 21

2.7 String potentiometers attached to modified control rods beneath the baggage compartment for measuring yaw and roll inputs . . . . . . . . . . 22

2.8 String potentiometer mounted to the aft side of the B-bulkhead for measuring pitch inputs ....................... 23

2.9 Instrument panel of a Katana at OAS . . . . . . . . . . . . . . . . . . . . . 23

2.10 The original, unpopulated aluminum instrument panel . . . . . . . . . . 24

2.11 A Flight Illusion altimeter and central module board . . . . . . . . . . . 25

2.12 Flight Illusion flight instruments installed in the KatanaSim instrument panel 26

2.13 Flight Illusion avionics stack installed in the KatanaSim instrument panel . 27 
2.14 A SimKits aircraft gauge, customized to act and look like a manifold pres-

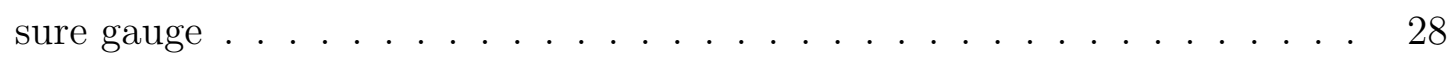

2.15 SimKits engine instruments installed in the KatanaSim instrument panel . 29

2.16 Klixon 3SB series simulator circuit breaker . . . . . . . . . . . . . . 30

2.17 Simulated circuit breakers installed in the KatanaSim instrument panel . . 31

2.18 Original switches, ignition, and flap controller installed in KatanaSim instrument panel . . . . . . . . . . . . . . . . . . . . 31

2.19 Populated KatanaSim instrument panel . . . . . . . . . . . . . . . . . . 32

2.20 The Logitech Z523 speaker system . . . . . . . . . . . . . . . . . . . 33

2.21 Immersive visual system concept sketch and the Katana canopy . . . . . 33

2.22 View of the KatanaSim showing the projection screen and mounting frame, as well as the projector mounted on the top of the fuselage $\ldots \ldots \ldots$. . . 35

2.23 The brake system of a Katana . . . . . . . . . . . . . . . . . . . 37

2.24 Brake lines, valves, and pressure sensors secured to the base of the KatanaSim 38

2.25 Half of the adjustable haptic feedback system for roll inputs found underneath the KatanaSim seats at the base of the control columns $\ldots \ldots$. . . 42

2.26 Half of the adjustable haptic feedback for pitch inputs located on the aft side of the B-bulkhead and attached to the elevator control rod; the other half of the system is located on the forward side of the bulkhead . . . . . .

2.27 Electrical setup on the base of the KatanaSim: two Arduino Mega 2560 microcontrollers, three power sources, and multiple reed relays and voltage

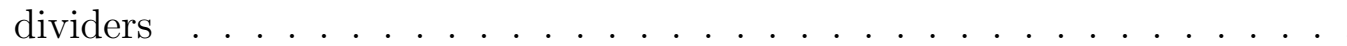

2.28 Centre console with pull knobs for carburetor heat, cabin heat, choke, and parking brake; below are the propeller pitch and throttle levers . . . . . . .

2.29 Top view of the potentiometer box on the front shelf of the engine firewall 46

2.30 Layout of the KatanaSim computer architecture and electrical components 
2.31 MOOG 6DOF 2000E motion base located in the Applied Dynamics Laboratory at Carleton University, used to provide motion cueing for the KatanaSim 48

3.1 Equipment mount to be installed in the baggage compartment during flight

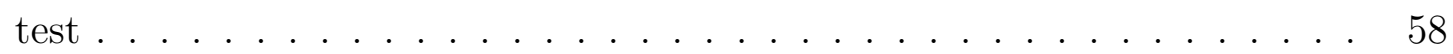

3.2 Left: Equipment mount secured in baggage compartment just prior to takeoff. Right: One corner of the equipment mount, secured with both a zip tie and parachute cord tied with a turn and three half hitches $\ldots \ldots \ldots$

3.3 String potentiometer attached to right co-pilot rudder pedal to record rudder movements during flight test . . . . . . . . . . . . 60

3.4 Custom-built clamps used for string potentiometer installation on underside of instrument panel . . . . . . . . . . . . . . . . . . . 61

3.5 String potentiometers installed under the instrument panel in front of the co-pilot to record control column movements during flight test . . . . . . .

3.6 Circuit diagram for the three string potentiometers, the power source, and the voltage dividers . . . . . . . . . . . . . . . . . . . . . 62

3.7 The fully-equipped Katana taxiing out for the first flight test . . . . . . . . 69

3.8 Image from the GoPro video recording of the flight test $\ldots \ldots \ldots \ldots$

3.9 Control column design showing pin joints . . . . . . . . . . . . . . 72

3.10 Front and side view of control column model used for post-processing of data to acquire control column position during flight test $\ldots \ldots \ldots$. . 73

3.11 Validation test results showing the discrepancy between the calculated control positions and the actual location of the rectangular perimeter of the range of the control column $\ldots \ldots \ldots \ldots \ldots \ldots \ldots$

3.12 Validation test results using tuned geometry data showing the alignment of the calculated data points with the rectangular perimeter of the range of the control column $\ldots \ldots \ldots \ldots \ldots \ldots \ldots$ 
4.1 A screenshot of Plane Maker showing the user interface for defining the lifting surfaces of an aircraft . . . . . . . . . . . . . .

4.2 Optotrak sensor tower (left) and stylus used to measure the airfoil coordinates of a Katana wing (right) . . . . . . . . . . . . . . 88

4.3 Wortmann FX63-137/20 airfoil profile as measured using the Optotrak Coordinate Measurement Machine . . . . . . . . . . . . . . . . . 88

4.4 Airfoil Maker polars for the Wortmann FX63-137/20 airfoil . . . . . . . . . 89

5.1 Three-dimensional definition of angular and linear velocities, forces, and axes in a body-fixed coordinate system . . . . . . . . . . . . 106

5.2 System consisting of a cart with spring and damper with an initial applied force, used for the creation of a simple curve-fitting GA . . . . . . . . . . . 121

5.3 Sliding pendulum system used as a test case in the development of the curve fitting genetic algorithm . . . . . . . . . . . . . . . . 123

5.4 Plot of linear acceleration $\dot{u}$ versus time, for calculated data from the GA and reference data from X-Plane in two dimensions . . . . . . . . . . . 128

5.5 Plot of linear acceleration $\dot{w}$ versus time, for calculated data from the GA and reference data from X-Plane in two dimensions . . . . . . . . . . . . 129

5.6 Plot of angular acceleration $\dot{q}$ versus time, for calculated data from the GA and reference data from X-Plane in two dimensions . . . . . . . . . . 130

5.7 Plot of linear acceleration $\dot{u}$ versus time, for calculated data from the GA and reference data from X-Plane in three dimensions . . . . . . . . . . 131

5.8 Plot of linear acceleration $\dot{v}$ versus time, for calculated data from the GA and reference data from X-Plane in three dimensions . . . . . . . . . . 131

5.9 Plot of linear acceleration $\dot{w}$ versus time, for calculated data from the GA and reference data from X-Plane in three dimensions . . . . . . . . . . . 132 
5.10 Plot of angular acceleration $\dot{p}$ versus time, for calculated data from the GA and reference data from X-Plane in three dimensions . . . . . . . . . . 132

5.11 Plot of angular acceleration $\dot{q}$ versus time, for calculated data from the GA and reference data from X-Plane in three dimensions $\ldots \ldots$. . . . . 133

5.12 Plot of angular acceleration $\dot{r}$ versus time, for calculated data from the GA and reference data from X-Plane in three dimensions $\ldots \ldots$. . . . . 133

F.1 Effect of CG location on take-off climb performance . . . . . . . . . . 174

F.2 Effect of wing span on take-off climb performance . . . . . . . . . . . 174

F.3 Effect of maximum available engine power on take-off climb performance - 175

F.4 Effect of CG location on straight and level flight performance . . . . . . 175

F.5 Effect of wing span on straight and level flight performance . . . . . . . 176

F.6 Effect of maximum available engine power on straight and level flight performance . . . . . . . . . . . . . . . . . . 176 


\section{List of Tables}

2.1 Forces required by the pilot on the control column handle to achieve gentle and aggressive roll and pitch angles . . . . . . . . . . . . . . . . 41

2.2 Kinematic capabilities of a MOOG 6DOF 2000E motion base . . . . . . 47

3.1 Flight test manoeuvres including flight time . . . . . . . . . . 56

3.2 Equipment required for recording flight test data . . . . . . . . . . 57

4.1 Effect of CG location on take-off climb performance . . . . . . . . . . 93

4.2 Effect of wing span on take-off climb performance . . . . . . . . . . . . 94

4.3 Effect of maximum available engine power on take-off climb flight performance 95

4.4 Effect of CG location on straight and level performance . . . . . . . . . 96

4.5 Effect of wing span on straight and level flight performance . . . . . . . 96

4.6 Effect of maximum available engine power on straight and level flight performance . . . . . . . . . . . . . . . . . . 97

5.1 Nomenclature for three-dimensional aircraft analysis . . . . . . . . . 106 


\section{Notation}

$\beta$

$\delta_{a}$

$\delta_{e}$

$\delta_{r}$

$\delta_{T}$

$\eta$

$\theta$

$\Phi$

$\varphi$

$\omega$

A

$A_{0}$

$\dot{A}$

$\Delta A$

$A C$

$F_{i}$

H

$I_{y}$

L

$L_{i j_{k}}$

L1

L2

M
Angle of roll deflection of the control column from the neutral position

Aileron deflection

Elevator deflection

Rudder deflection

Thrust

Control vector

Angle of pitch deflection of the control column from the neutral

position (Chapter 3); Pitch angle of the aircraft (Chapter 5)

Roll angle of the aircraft

Yaw angle of the aircraft

Angular velocity

Arbitrary vector

Reference condition of variable $A$

First time derivative of variable $A$

Change in variable $A$

Coordinate system local to the aircraft

Force in the $i$ direction

Angular momentum vector

Moment of inertia with respect to the pitch axis of the aircraft

Aerodynamic moment about the roll axis of the aircraft

Position of Point $i$ with respect to Point $j$ in the $k$ direction

Coordinate system local to Link 1 of the control column model

Coordinate system local to Link 2 of the control column model

Moment 
Aerodynamic moment about the pitch axis of the aircraft aerodynamic moment $\mathrm{M}$ per unit moment of inertia mass

$p$

$q$

$\mathbf{x}$

$x$
Aerodynamic moment about the yaw axis of the aircraft

Angular velocity about the roll axis of the aircraft

Angular velocity about the pitch axis of the aircraft

Position of Point $i$ with respect to Point $j$, in coordinate system $k$

Radius of sphere centered at Point $i$

Angular velocity about the yaw axis of the aircraft

Transformation matrix from coordinate system $i$ to coordinate system $j$

Linear velocity along the roll axis of the aircraft

Total velocity

Linear velocity along the pitch axis of the aircraft

Linear velocity along the yaw axis of the aircraft

Aerodynamic force along the roll axis of the aircraft

Stability derivative representing the effect of variable $i$ on force $\mathrm{X}$

per unit mass

State variable vector

Roll axis of the aircraft

Aerodynamic force along the pitch axis of the aircraft

Stability derivative representing the effect of variable $i$ on force Y

per unit mass

Pitch axis of the aircraft

Aerodynamic force along the yaw axis of the aircraft 
$Z_{i} \quad$ Stability derivative representing the effect of variable $i$ on force $\mathrm{Z}$ per unit mass Yaw axis of the aircraft 


\section{Chapter 1}

\section{Introduction}

\section{$1.1 \quad$ A Brief History of Flight Simulators}

In December of 1903, Orville and Wilbur Wright successfully flew the first manned powered aircraft and launched a new era of aviation. With the proliferation of aircraft in the years that followed came the advent of flight training schools. The very first training programs began with a familiarization flight, and then the students were allowed to practice rudder control while taxiing. Next, they practiced elevator control while their aircraft did short hops along an airfield. Finally, the students were allowed to take a full flight in the aircraft [1]. In these earliest of days, it became clear even to the Wright brothers that flight training could be made safer and less expensive if some portion of the training could be completed in ground-based trainers. The Wright brothers began experimenting with fuselage sections mounted on pivots and actuated manually by assistants outside the simulator. The fidelity of these motions was limited to the knowledge and ability of the people controlling the movements $[2]$.

The Sanders Teacher, developed in 1910, was one attempt to increase the functional fidelity of the primitive simulator. The Teacher was an aircraft frame mounted on a 
universal joint installed in an exposed, outdoor area in order to allow trainees to practice controlling the aircraft when wind conditions allowed [1]. This unfortunate reliance on weather eventually spurred the creation of flight trainers that relied on neither weather nor physical labour to create simulator motion responses.

Perhaps one of the most well known early flight trainers was the Link Trainer, first patented in 1930, which used pneumatic mechanisms from player pianos to exert pressure on the control column and rudder pedals [2]. It also had an electrically-driven suction pump which fed the control valves operated by the flight controls, and a motor-driven device which produced changes in the attitude of the cockpit. Despite the Great Depression, the Link Trainer was sold to flight schools around the world including England, Japan, France, Germany, and the USSR. In 1937, American Airlines purchased a Link trainer making it the first airline to use a flight trainer as part of its training program [1].

World War II was a great catalyst for aerospace and simulator technology. The addition of complex systems and the tendency of military pilots to operate their aircraft at the edge of the performance envelope created a sense of urgency for safer and more effective ground-based training capabilities. A Spitfire trainer, called the Hawarden Trainer, was made using a Spitfire fuselage, and many manufacturing companies, including Link, began to incorporate type-specific instrument layouts in their designs [1].

Flight trainers were used by the UK military not just as training tools, but also for behavioural research and aircrew selection. Using a modified aircraft fuselage, test subjects would be assessed on their competence as pilots, susceptibility to distraction, and rate of acquisition of flying skill, and were subsequently assigned to different forms of flying service. The Royal Air Force Institute of Aviation Medicine (RAF-IAM) acquired its first flight trainer for research purposes in 1955. The trainer was configurable either as a Mk 4 Hawker Hunter or as a generic single seat cockpit. [3]*

\footnotetext{
${ }^{*}$ Citation outside of punctuation at end of paragraph denotes reference use throughout the paragraph.
} 
Around the mid-century mark, flight simulators were recognized as potential tools to aid in the design of future aircraft. At the RAF-IAM in the 1950s, test-subjects were asked to evaluate various types of attitude indicators and other instruments [3]. With the development of analog computers after WWII came the next leap forward in simulation technology, and the advent of the word "simulator" [2]. Computers allowed the equations of motion of the aircraft to be solved and a proper flight model to be developed for the first time. A simulator was then able to predict the behaviour of the aircraft, rather than use empirical duplications. In 1944, MIT began work on the first computerized flight simulator capable of providing instantaneous responses to flight control inputs; it was called the MIT Whirlwind Digital Computer [2].

Flight model development in the 1950s was significantly hindered by the lack of accurate performance data. As more airframe and engine manufacturers began performing more extensive flight testing, a significant increase in analogue computing hardware was required to keep up. The law of diminishing returns dictated that a new technology was needed as the cumulative errors caused by the additional hardware began to outweigh the increased accuracy for which it had been introduced. The most successful breakthrough was the use of digital computers in the development of the Mark I simulator by Link in the early 1960s. The commercial aviation industry soon began to take notice; they wanted to be able to simulate in-flight emergencies such as fires and system failures and this new generation of simulator could do just that. Mark I simulators were sold to the American military and most major airlines around the world. [1]

With the advancement of digital computing and flight modelling in the decades that followed, present day flight simulators have become irreplaceable in their use in aircraft design. Aviation manufacturers are pressured to find cost-effective ways to design, build, and test their aircraft in increasingly short time frames and the highly accurate predictive flight models enable manufacturers to do just that [4]. Using these multi-million dollar 
simulators, engineers can evaluate flight deck design and resolve issues involving human factors while pilots can give feedback on handling characteristics for an aircraft that does not yet exist. Aircraft marketing strategies take advantage of simulators to give their potential customers previews of their product. Wind shear certification testing is considered too dangerous to be performed in real aircraft as the abrupt changes in wind speed and direction can cause extreme losses in airspeed and altitude during take off and landing; wind shear certification is now completed using flight simulators [2].

Commercial flight training has also been revolutionized by these high-cost high-fidelity type-specific simulators. Under the Federal Aviation Administration's Advanced Qualification Program pilots are allowed to earn type certifications after completing all required training hours in certified flight simulators; it is now standard for commercial airline pilots to fly their first flights as First Officers of passenger-laden aircraft $[2,5]$. These airline flight simulators are typically built by companies such as CAE (Montreal, Canada), Mechtronix (Montreal, Canada), and SimIndustries (Sassenheim, The Netherlands) and are so expensive that many commercial airlines opt to rent time in simulators rather than purchase their own. Pan Am International Flight Academy (Miami, USA) is one such company that owns 30 different full flight simulators and flight training devices and sells training time in them [6]. Airlines around the world send their pilots to the academy, in particular for time on the Boeing 737-800 simulator, and pay upwards of $\$ 700$ per hour for the privilege [7]. Some airlines, like WestJet (Calgary, Canada), do purchase their own simulators but recoup the cost by renting out simulator time to other airlines [7]. These airline simulators are usually certified to the highest levels by Transport Canada (TC) or the Federal Aviation Authority (FAA) and are built using customized parts designed by the simulator manufacturers. The simulators may use extended field-of-view collimated displays for enhanced visual cueing as well as producing all cockpit sounds, motions, and instrument presentations to produce a completely realistic experience for the simulator 
occupants [8]. One such flight simulator built by Mechtronix can be seen in Figure 1.1.
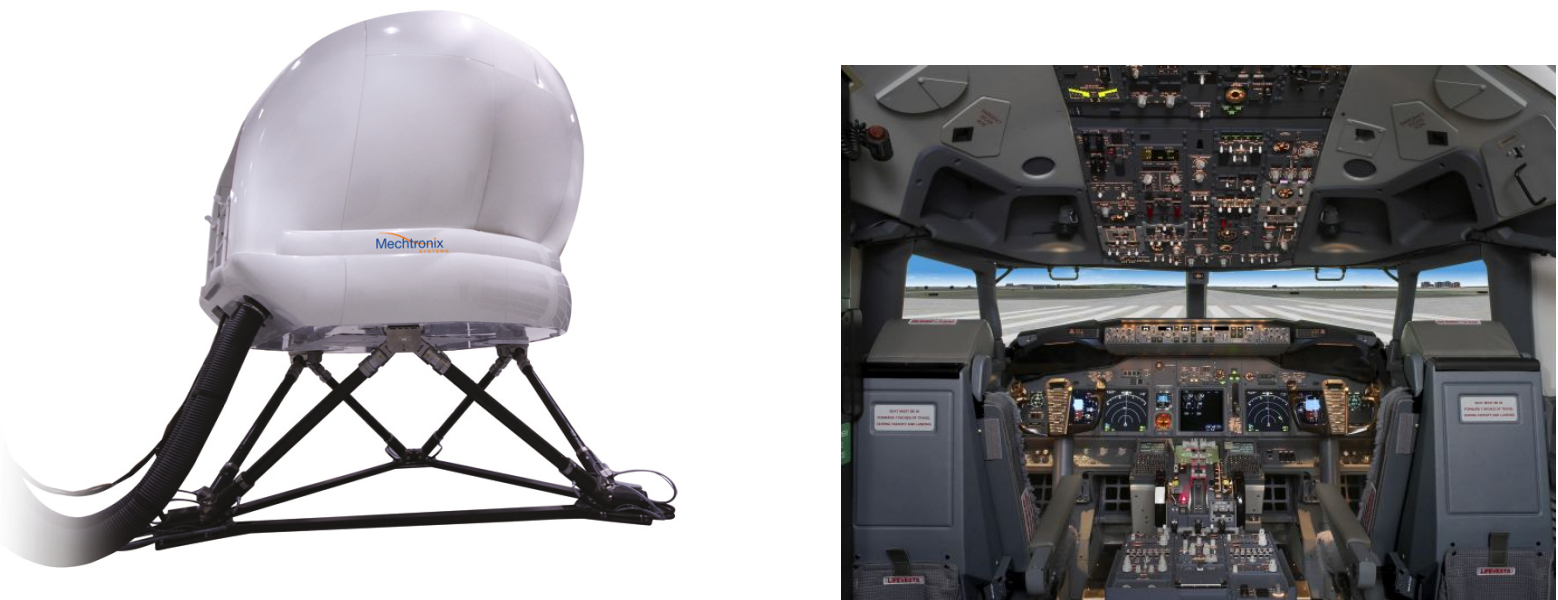

Figure 1.1: Mechtronix full-flight simulator $\mathrm{X}^{T \mathrm{TM}} 737-800$ exterior (left) and interior (right). Photographs courtesy of Mechtronix.

\subsection{Simulation Fidelity and Certification}

Unfortunately, simulator training is not always beneficial. Simulators that are poorly designed or used incorrectly can result in negative transfer, in which the students develop habits or learn procedures which could negatively affect their performance or safety in an actual aircraft [9]. To ensure negative transfer does not occur, it is important that the fidelity of the simulator be considered during development. Fidelity can be divided into three areas: functional, environmental, and physical. Functional fidelity can be achieved by ensuring the simulator control and flight models mimic those of the aircraft. Environmental fidelity encompasses the sounds, motions, and general ambience of the simulator. Physical fidelity requires that the spatial, tactile, and appearance aspects of the simulator be similar, or identical to, those of the desired aircraft. Type-specific simulators have much higher physical fidelity than generic simulators. Students forced to train in generic simulators do not gain the same familiarity with the cockpit instruments and flight controls as they 
would if they were to train in a type-specific flight simulator [10].

Because of the prolific use of simulators for commercial airline training, certification of a flight simulator is required before it can be used in a training curriculum. Simulator certification is regulated in Canada by TC and in the USA by the FAA. TC divides flight simulators into two main groups: Flight Training Devices (FTDs) and Aircraft Simulators, also called Full Flight Simulators; and defines them as follows in the Aeroplane and Rotorcraft Simulator Manual [11]:

Flight Training Device: a replica of an aircraft's instruments, equipment, panels and controls in an open flight deck area or an enclosed aircraft cockpit, including the assemblage of equipment and programs necessary to represent the aircraft in ground and flight conditions to the extent of the systems installed in the device; does not require a force (motion) cueing or visual system; is found to meet the criteria outlined in this manual for a specific flight training device level; and in which any flight training event or flight checking event is accomplished.

Aircraft Simulator: a full size replica of a specific type or make, model and series aircraft cockpit, including the assemblage of equipment and computer programs necessary to represent the aircraft in ground and flight operations, a visual system providing an out-of-the-cockpit view and a force cueing system that is in compliance with the standards as specified in this manual.

FTDs are classified as Levels 1 through 6 based on their equipment and software fidelity, while full flight simulators are classified as Levels A through D. The standards required for each of these levels are found in the Aeroplane and Rotorcraft Simulator Manual [11]. 


\subsection{Benefits of Flight Simulator Training}

Quality of training can be greatly improved through the incorporation of simulator time into training schedules leading to more comprehensive training programs. Pilots can be trained systematically on tasks ranging from cockpit familiarization through to compound failure recovery, in a safe environment. Tasks, like take-offs or landings, can be performed repeatedly without the need to fly a full circuit or wait for other airport traffic to clear the area. Dangerous manoeuvres and emergency procedures which could never be safely practiced in flight can be reviewed in a simulator without fear of consequence. Cost effectiveness is another attractive quality of simulator training. Flight training can proceed without regard for fuel and maintenance costs, airport fees, and other overhead costs. Pilots can also train regardless of weather conditions which could otherwise result in potentially costly delays. With an increase in simulation time incorporated into training curricula, students have the potential to earn their licenses and type certifications faster and more safely than ever before. [12]

\subsubsection{Simulator Use During Ab-initio Training}

The benefits of flight simulation training have long been recognized and put to use in the training of military and commercial pilots and it has been widely accepted that simulated flight time could be used to supplement and enhance a typical training curriculum [9]. One particular study was conducted at Embry-Riddle Aeronautical University (Daytona Beach, Florida) in which 68 ab-initio student pilots (student pilots with no flight experience) followed an experimental flight training curriculum consisting of $40 \%$ flight time in a Cessna 172SP and 60\% time in a high-fidelity Level 6 Cessna 172 FTD manufactured by Frasca International, Inc (Illinois, USA) [9]. The effectiveness of training was measured using the Transfer Effectiveness Ratio (TER) defined as 


$$
\mathrm{TER}=\frac{\mathrm{C}-\mathrm{E}}{\mathrm{E}_{f t d}}
$$

where $\mathrm{C}$ is the number of iterations a control group took to successfully complete a task in flight, $\mathrm{E}$ is the number of iterations an experimental group took to do the same, and $\mathrm{E}_{f t d}$ is the number of iterations the experimental group took to complete the task when in the FTD. The resulting TER is an indication of how many iterations in the aircraft are saved by doing one iteration in the FTD. In all but one of the 34 training tasks studied, positive TERs were found ranging from 0.01 to 2.1, and 20 of the manoeuvres required "significantly" fewer iterations after FTD training. Diversions were the sole task which resulted in negative training transfer from time in the FTD with a TER of -0.02 . It was hypothesized that this was because diversions were made more difficult by FTD training because of their reliance on an abundance of outside visual cues and landmarks which were not sufficiently provided.

A similar study by the American military evaluated military ab-initio flight students (students with no previous flight training) and found an overall TER of 0.55. No negative training transfer was apparent, no students were eliminated nor made to repeat any part of the training programs, and the cost of training was significantly reduced [5].

\subsubsection{Factors Affecting Transfer of Training}

From the previously mentioned studies, five influential factors were identified as having impacts on the transfer of training from the FTDs: visual fidelity, difficulty of task, procedural similarity, visual scanning and response, and air mass modelling [9]. 


\section{Visual Fidelity}

TERs are found to be directly linked to the quality of the visual system [13]. Higher TERs were achieved when detailed and well-developed three-dimensional graphics were used. The visuals were particularly important for tasks where cues taken from landmarks and other visual reference points were highly important, such as circuit training, forward slips, and emergency approaches.

\section{Procedural Similarity}

The fidelity of the FTD to the aircraft is important in achieving high FTRs. High physical and cognitive fidelity are of the utmost importance. High-level FTDs are often made with replica cockpits to ensure high physical fidelity. In particular, the introduction of a simulated air traffic controller and other air traffic greatly improves the cognitive fidelity and, coincidentally, the TERs for procedure-based tasks such as pre-flight checks, traffic patterns, and radio communications [13].

\section{Difficulty of Task}

The difficulty of the task also appeared to be linked to the TER achieved, as notoriously difficult tasks such as take offs and landings on short or soft fields scored lower TERs than most other tasks.

\section{Visual Scanning and Response}

High TERs were recorded for slow flight and stalls; it was hypothesized that this was due to the increased instrument scanning aptitudes of students who trained in the FTD. In flight, students could receive physical cues and sensations from the aircraft motions, whereas in the simulator the students had to be reliant on instrument training. 


\section{Air Mass Modelling}

In relatively simple tasks such as straight and level flight or constant airspeed climbs, only small TERs were recorded. These tasks can be greatly impacted by buffeting from air currents, thermals, and other forms of turbulence. The simulator software used in [9] was deemed insufficient in its ability to model these air effects, thus negating most of the training benefit of these tasks.

\subsection{Small Aircraft Simulator Research}

The level of technology and fidelity is very high for commercial and military applications, but there is a need in the industry to increase the accessibility of this technology to those training for their private pilot licenses (PPLs). Most flight schools will train their student pilots on single-engine, recreational aircraft like Cessna 172s and Diamond Katanas. These flight schools usually operate on limited budgets and cannot afford to pay for the highfidelity FTDs and full flight simulators that would improve the quality of their ab-initio training programs so significantly. The cost of a high-fidelity FTD usually starts at about three times the price of a new aircraft, and though they have been shown to offer four times the training value [5], flight clubs often choose to go without.

Research groups around the world have taken varying approaches to the absence of flight simulators and FTDs in ab-initio pilot training. As discussed in Section 1.3.1, EmbryRiddle Aeronautical University (ERAU) has done extensive research on the use of FTDs in flight training courses, particularly ab-initio training. Research groups at ERAU developed a new curriculum in which students spend $60 \%$ of their flight training time in a Level 6 certified Cessna 172SP FTD built by Frasca International [12]. The training effectiveness was evaluated and found to be significant for the majority of the flight tasks examined [9]. After the training effectiveness was ascertained, research groups at ERAU continued to 
study what aspects of the FTD systems were the most influential in providing positive training experiences; these factors were discussed previously in Section 1.3.2. The extensive research by ERAU has greatly improved the understanding of how effective simulator training can be in ab-initio training and how the experience can be optimized; however, this research has done little to benefit those flight schools who are unable to access such high-fidelity FTDs and flight simulators.

Researchers at the School of Mechatronics Engineering at the Harbin Institute of Technology (Harbin, China) recognized the specific industry need for affordable simulation technology $[14,15]$. A Boeing 737-300 flight simulator was developed using a PC-based design and focusing on commercial off the shelf (COTS) software components in an effort to reduce design costs. Eleven PCs, three projectors, and two speakers formed the bulk of the hardware components. A cockpit was created in which gauges and avionics were substituted with LCD screens, though it can be noted that a Boeing 737 is generally equipped with a large number of electronic instruments so the LCD screens did not greatly diminish the physical fidelity of the simulator cockpit. The flight model was designed in detail using MATLAB/Simulink, the visual environment was created in MultiGen Creator, and both the visual and audio environments were driven by MultiGen Vega. The performance of the flight model was compared to flight test data and found to be within certification limits for an FTD (level unspecified) though it was noted that the cockpit design lacked an authentic feel. A compatible motion cueing system was virtually designed but not implemented. The development of this simulator was an example of how the development costs of a high-fidelity simulator can be greatly reduced by taking advantage of low-cost highperformance PCs and COTS hardware; however, the simulator was not modelled after a small aircraft of the type used in small flight schools for ab-initio training. 


\subsection{The KatanaSim Project}

The KatanaSim project is an attempt to create an accessible alternative to currently available small aircraft FTDs and full-flight simulators. Simulator manufacturers like Frascas International have developed high-fidelity small aircraft FTDs but the cost of these simulators is prohibitive to small flight training facilities. The KatanaSim is a low-cost type-specific high-fidelity flight simulator prototype designed for use as part of an ab-initio flight training program. Just like the PC-based simulator designed at the Harbin Institute of Technology, the KatanaSim is a PC-based design but the focus on COTs components was expanded to include not just software, but also aircraft instruments and cockpit components. Original aircraft parts were used to supplement the hardware wherever practical. The KatanaSim was built with the long-term goal of certification as an FTD, but was also tested on a motion base to assess the benefits and feasibility of certification as a full-flight simulator.

The KatanaSim project is a partnership between the Carleton University Applied Dynamics Laboratory (ADL) and two companies located in Ottawa, Canada. Ottawa Aviation Services (OAS) Flight Centre (Ottawa, Canada) provided financial support, simulator building materials in the form of aircraft parts, and guidance from their staff and flight instructors. Additional financial support was provided by B-Con Engineering, an optical engineering company. As OAS has a small fleet of DA20-A1 Diamond Katanas ${ }^{\dagger}$, it was decided that the KatanaSim would be made type-specific to this model in order to complement the OAS PPL training program.

Katanas are designed and built by Diamond Industries located in London, Canada. A Katana (shown in Figure 1.2) is a single-engine, low-wing, T-tail aircraft famous for its sleek white composite form made of glass- and fiber-reinforced plastic. The aircraft has non-retractable tricycle landing gear with a castering nose wheel and a bubble canopy.

\footnotetext{
†Note: Subsequently in this document "Katana" refers specifically to the DA20-A1 model.
} 


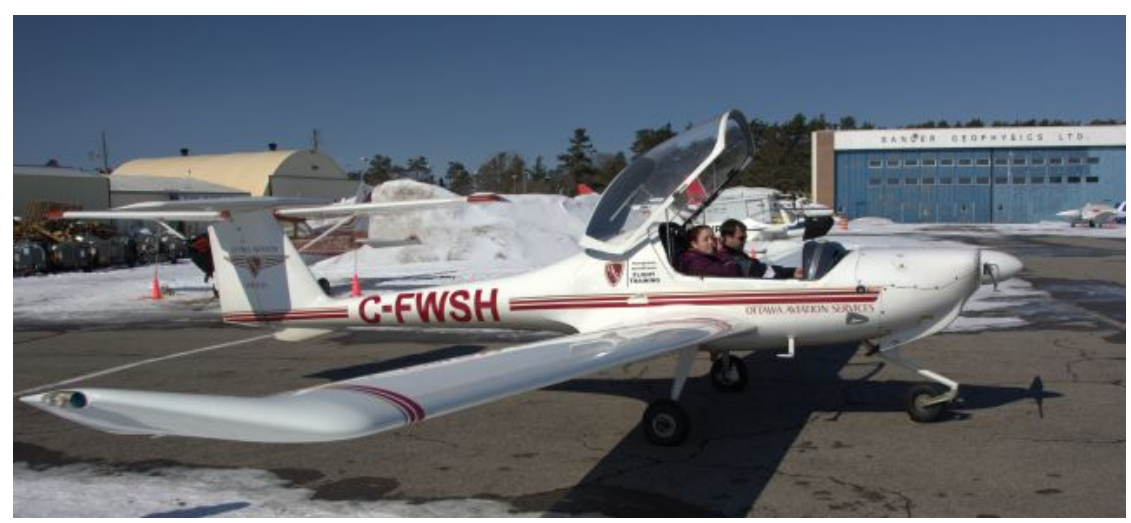

Figure 1.2: A DA20-A1 Diamond Katana.

With a maximum take-off weight of $730 \mathrm{~kg}$ (1609 lbs), a Katana seats only two: a pilot and co-pilot (or passenger) side by side. The aircraft is powered by the 80 HP Rotax 912 engine and has a cruise speed of $167 \mathrm{kph}$ (90 knots). While not an exceptionally powerful or large aircraft, they are a favourite for small flight training schools around the world. [16]

\subsection{Thesis Overview}

As the KatanaSim was a team project, multiple people contributed to the hardware development and flight testing described in Chapters 2 and 3. Where significant work was done by other people, explicit references are made.

\subsubsection{KatanaSim Hardware}

The KatanaSim Hardware chapter details the design decisions made in selecting and assembling the various systems. System and component descriptions include flight controls and brakes; instrument panel components; the audio, visual, and motion cueing systems; the haptic feedback system, and the simulator software. An overview of all hardware systems is provided, but as the KatanaSim project was a team effort within the ADL, this thesis will focus less on systems that were designed or implemented by consultants or other 
members of the KatanaSim Team.

\subsubsection{Flight Testing}

The Flight Testing chapter outlines the development of a minimally-intrusive test method for small aircraft to gather a full range of performance data. This methodology was developed in order to gather adequate data to fully evaluate and validate the KatanaSim flight model. Three flight plans were designed to gather performance data for a range of manoeuvres typically encountered in a PPL training program, as well as manoeuvres specifically designed to test stability characteristics of the aircraft. The flight test equipment is presented and the methods for post-processing the flight test data are explained. As the flight testing initiative was a joint project with two other research groups in the Mechanical and Aerospace Engineering department at Carleton University, less focus is placed on systems and equipment selected by other team members. A full description of the testing methodology can be found in the Carleton University Katana Flight Test Plan [17].

\subsubsection{Flight Model}

The Flight Model chapter describes the process by which a flight model for the KatanaSim was created using data gathered from a variety of sources including empirical measurements taken of the aircraft. Due to conflicting information in the published data, modifications had to be made to the flight model to increase the accuracy of the design. The advantages and disadvantages of blade element theory, the method by which the selected software predicts the flight performance of a given aircraft model, are discussed. The performance of the initial design of the flight model did not closely match the performance of the Katana observed during flight, leading to a sensitivity study in an attempt to determine a tuning method for the system. 


\subsubsection{Automated Flight Model Tuning}

Inspired by the need to tune the KatanaSim flight model to match the acquired flight test data, the penultimate chapter introduces the concept of flight model tuning using genetic algorithms. The development of a genetic algorithm, a heuristic optimization algorithm, is described using principles borrowed from natural selection and evolution. This algorithm is applied to a wide variety of curve fitting problems. The developed algorithm is then tested with simulated flight test data in order to tune the stability derivatives of the aircraft and prove the ability of the genetic algorithm to be used in flight model tuning. The results of the tuned performance curves are presented.

\subsubsection{Conclusion}

In the Conclusion, specific thesis contributions will be outlined and the final thoughts on the feasibility of the KatanaSim to fill the industry need for cost-effective, type-specific, small aircraft simulators will be presented. Future work on the hardware and flight model are outlined. 


\section{Chapter 2}

\section{KatanaSim Hardware}

\section{$2.1 \quad$ Overview}

The aim of the KatanaSim project is the development of a cost-effective, high-fidelity, typespecific flight simulator. In order to achieve this goal, the manufacturing and procurement methods for all components were carefully considered. It was decided that the simplest and perhaps most obvious way of building a type-specific flight simulator was to use original aircraft parts wherever practical in the assembly. This in turn guaranteed that a high level of physical fidelity could be achieved. In keeping with the goal of creating a flight simulator accessible for small fight clubs, the cost of the project was kept as low as possible by keeping the number of custom built parts to a minimum. The use of original aircraft parts from OAS also helped to keep the development cost low as these parts were readily available from OAS at no additional cost to the project. Where original components could not be used, either for reasons of safety or complexity, COTS components were implemented wherever possible. In the remaining cases, where custom solutions were required, these were designed and implemented. 


\subsection{Body and Support Structure}

Before the conception of the KatanaSim project, OAS had been in possession of a Katana, tail number C-FWNT, that was no longer airworthy. The aircraft had been in an accident in which the right main landing gear had been torn from the fuselage of the aircraft, and significant damage had been done to critical internal structures of the aircraft including the spar tunnel. Due to the nature of the composite materials used in the construction of the fuselage, it was not financially advantageous for OAS to have the aircraft repaired and they opted to purchase a replacement instead. This decision left OAS with key parts available for use in the KatanaSim, including the actual fuselage of the aircraft, as seen in Figure 2.1. This fuselage became the central structure of the simulator, ensuring not only that the interior geometry of the KatanaSim was identical to that of a Katana aircraft, but that students would be able to practice proper entry and egress techniques during their simulator sessions.
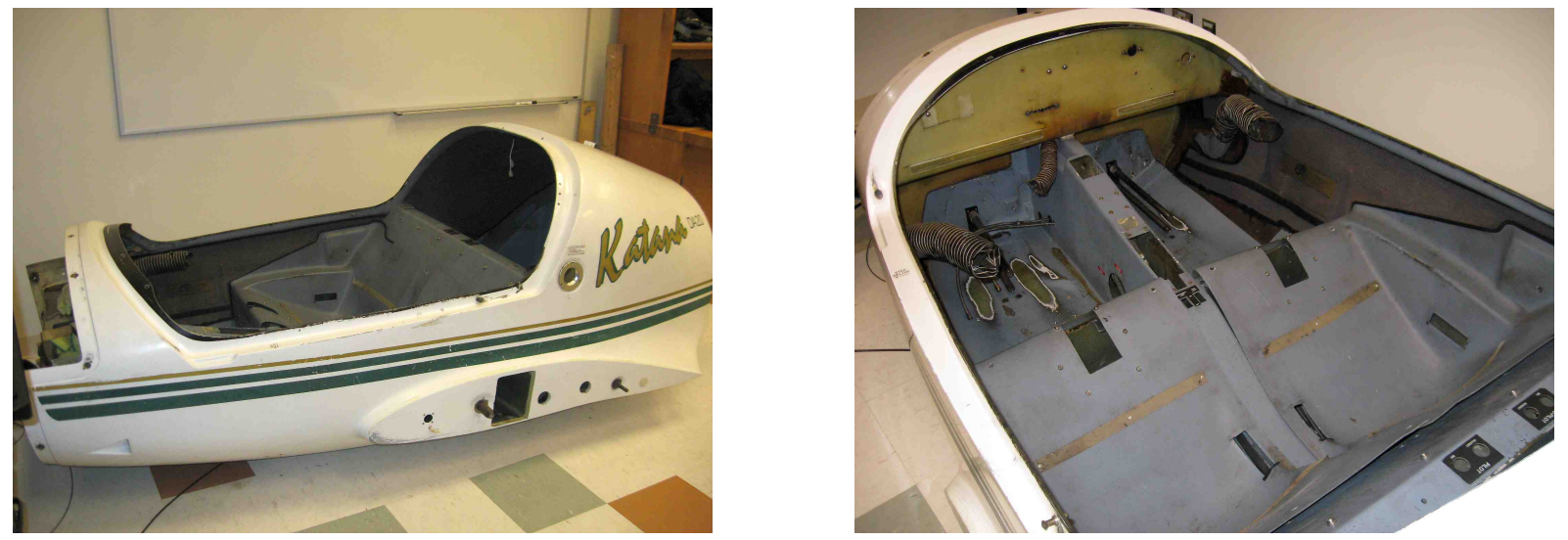

Figure 2.1: Katana fuselage, stripped bare and cut down to size.

The largest parts of the aircraft including the wings, landing gear, engine, and propeller were removed and the fuselage interior was stripped of all instrumentation, wiring, controls, and accessories. The tail boom and empennage of the aircraft was removed just aft of the baggage compartment leaving enough of the structure to support the possible integration 
of the aircraft canopy, discussed later in Section 2.6.

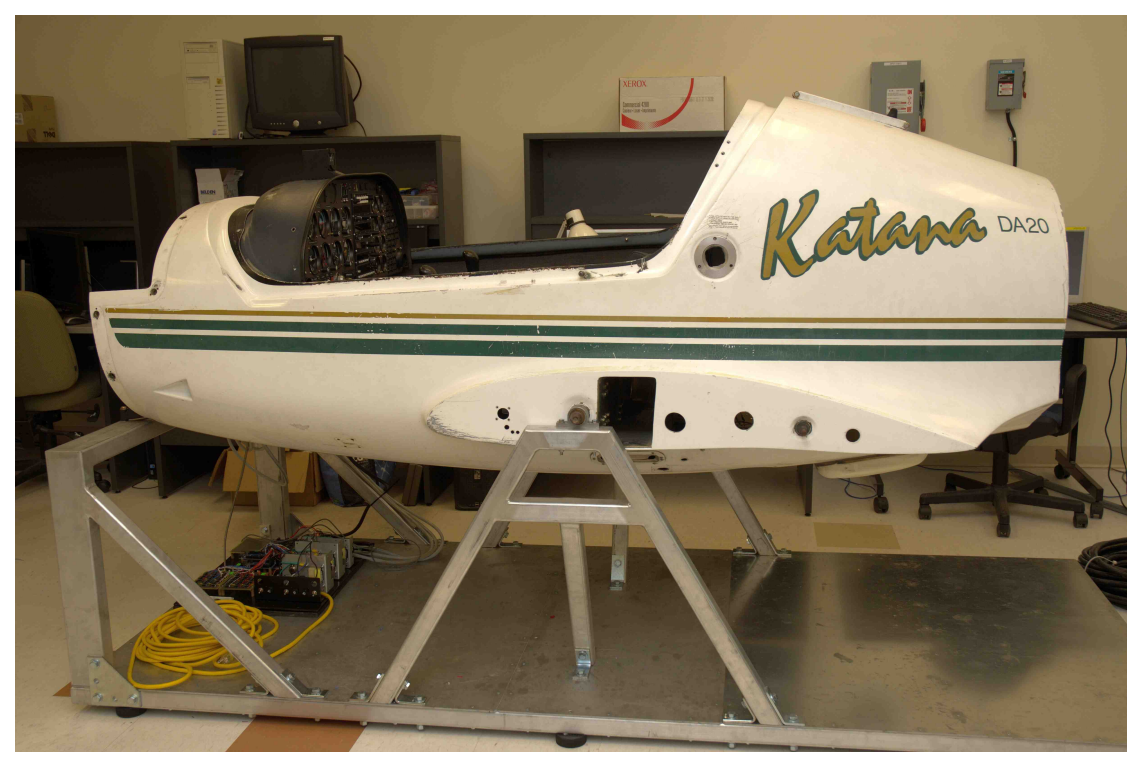

Figure 2.2: KatanaSim fuselage mount.

After the landing gear had been removed from the fuselage, a support structure was designed by Joel Spark and Michael James Leveille* to keep the fuselage stable and correctly oriented, as well as to provide a method of mounting the simulator to the MOOG 6DOF 2000E motion base (described in Section 2.11) during later stages of development. The support structure interfaces with the fuselage using connection points at the wing pins and the engine mounting points. These points ensure the fuselage is properly supported while allowing uninterrupted entry and egress to and from the simulator. Figure 2.2 shows the KatanaSim fuselage mounted on the support structure.

\subsection{Flight Controls}

Each seat in a Katana is equipped with a control column and a set of rudder pedals. The rudder pedals of the aircraft were reinstalled in their original configuration. In the aircraft,

${ }^{*}$ Research assistants in the Applied Dynamics Laboratory at Carleton University. 


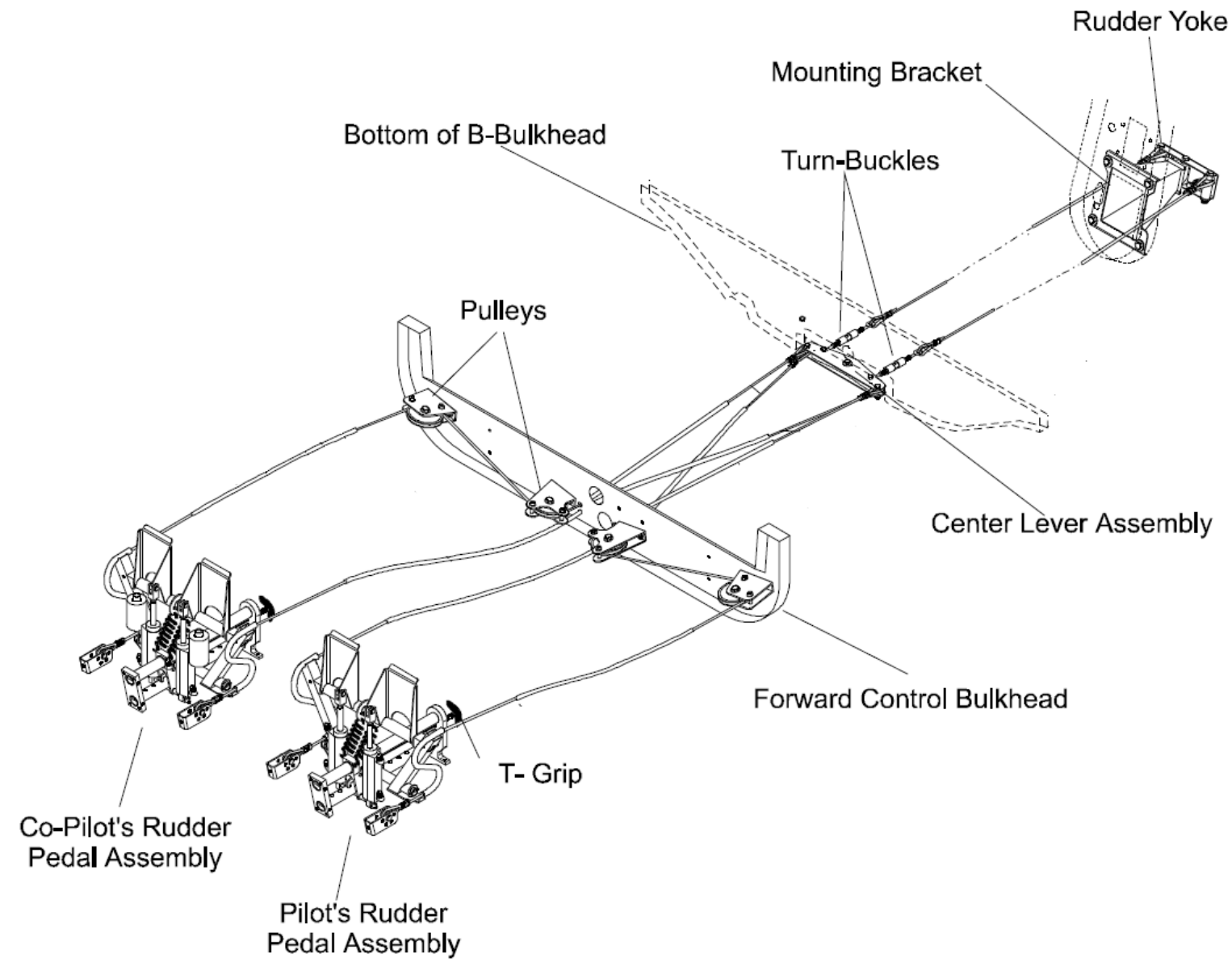

Figure 2.3: Diagram of the rudder assembly in a Katana aircraft from the Aircraft Maintenance Manual [18].

a cable and pulley system is used to transfer motion from the rudder pedals to the rudder itself; this system is shown in Figure 2.3. The foremost half of the system was installed as it would be regularly in a Katana with the cables from the pedals attached to the centre lever assembly located beneath the baggage compartment, in line with the B-bulkhead depicted in Figure 2.3. No rudder system components aft of the centre lever assembly were installed. Figure 2.4 has been included for additional reference as to the location of the bulkheads and baggage compartment.

Unlike the rudder system, the ailerons and elevators, controlling roll and pitch respectively, are actuated using control rods rather than cables and pulleys. Two sets of control 


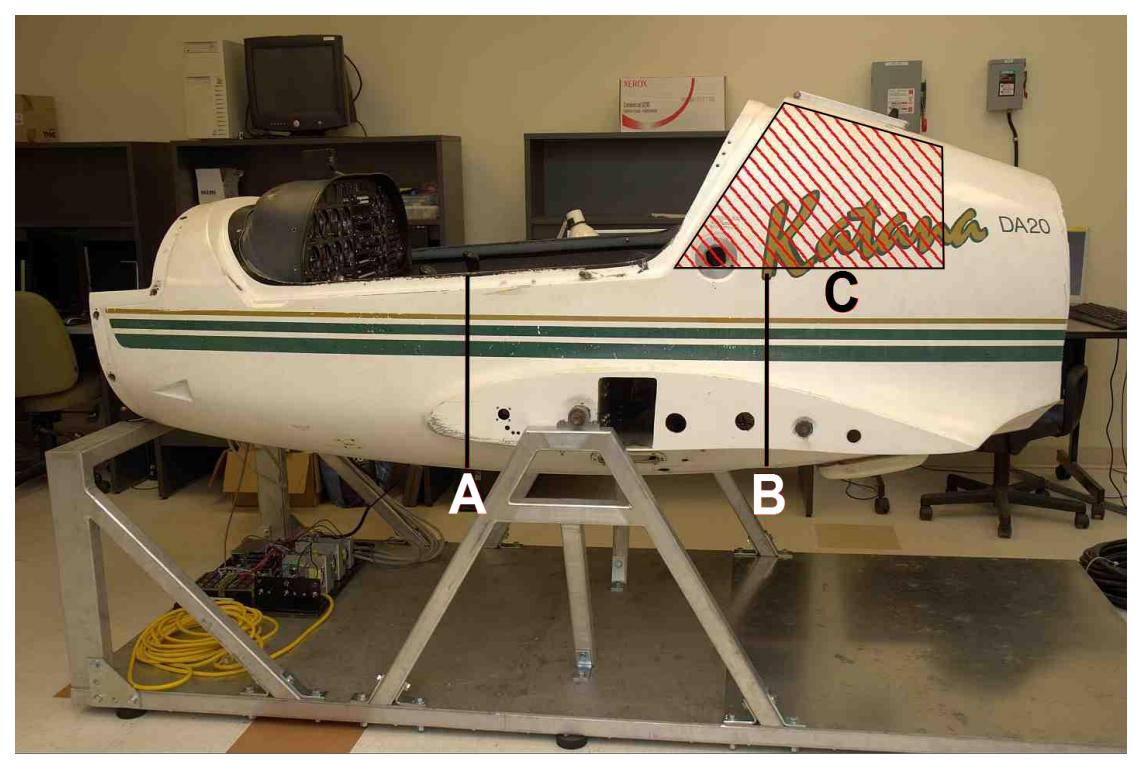

Figure 2.4: KatanaSim profile showing placement of the forward control bulkhead (Line A), B-bulkhead (Line B), and baggage compartment (Area C).

rod linkages, one each for roll and pitch control, are connected to the control column assembly. The elevator control system of a Katana can be seen in Figure 2.5. The dashed line in the figure represents where the control rod was shortened in the KatanaSim, just aft of the B-bulkhead in the rear section of the fuselage. No control rods were installed aft of this point.

The aileron control assembly can be seen in Figure 2.6; the dashed circle indicates the point after which no more control rods were installed in the KatanaSim. This point is located just forward of the B-bulkhead, beneath the baggage compartment.

Two string potentiometers were then installed as shown in Figure 2.7. One string potentiometer was attached to the centre lever assembly to measure yaw inputs and the second string potentiometer was attached to the aileron control rod to measure roll inputs. Both of these string potentiometers were located forward of the B-bulkhead, beneath the baggage compartment.

A third string potentiometer, shown in Figure 2.8, was installed on the back side of the B-bulkhead and was attached to the elevator control rod to measure pitch inputs. The 


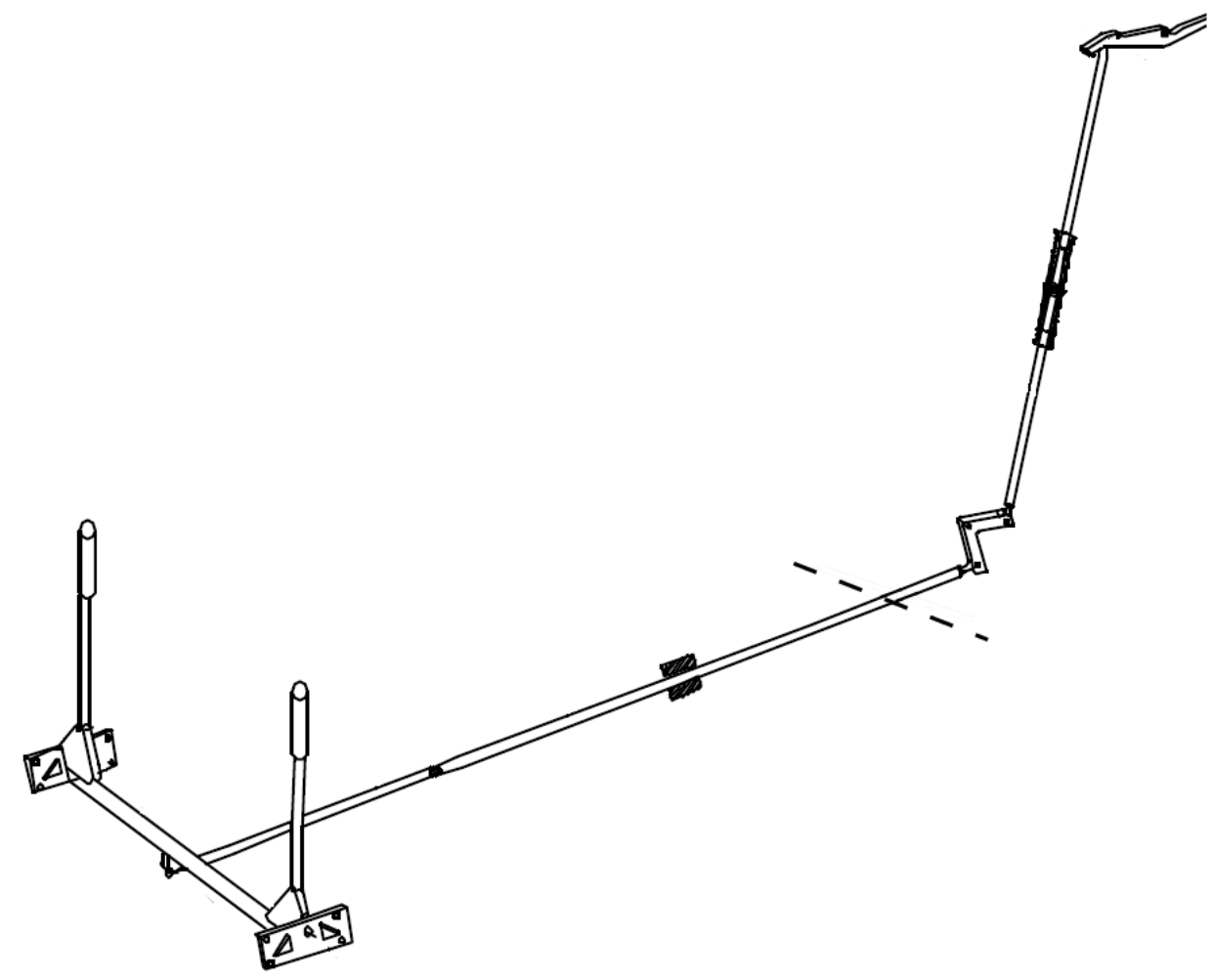

Figure 2.5: Elevator assembly in a Katana aircraft [18]; the dashed line depicts where the control rod was cut, just aft of the B-bulkhead. The remainder of the system was not installed in the KatanaSim.

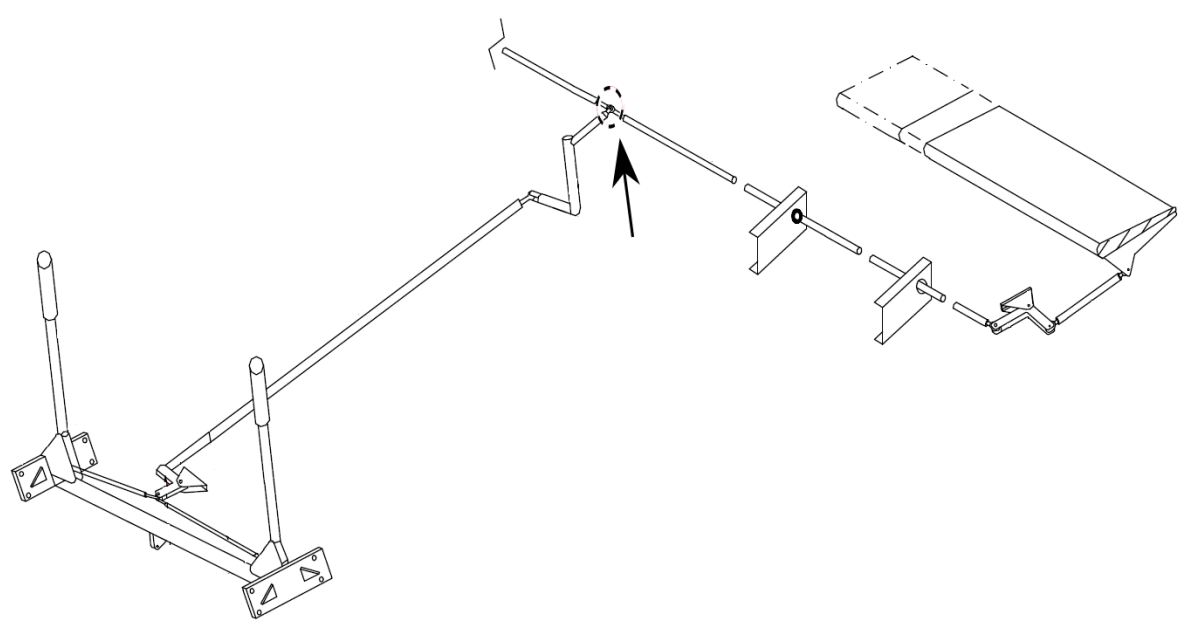

Figure 2.6: Aileron assembly in a Katana aircraft [18]; the red circle indicates the end point of the system installed in the KatanaSim, which is located just forward of the B-bulkhead. 


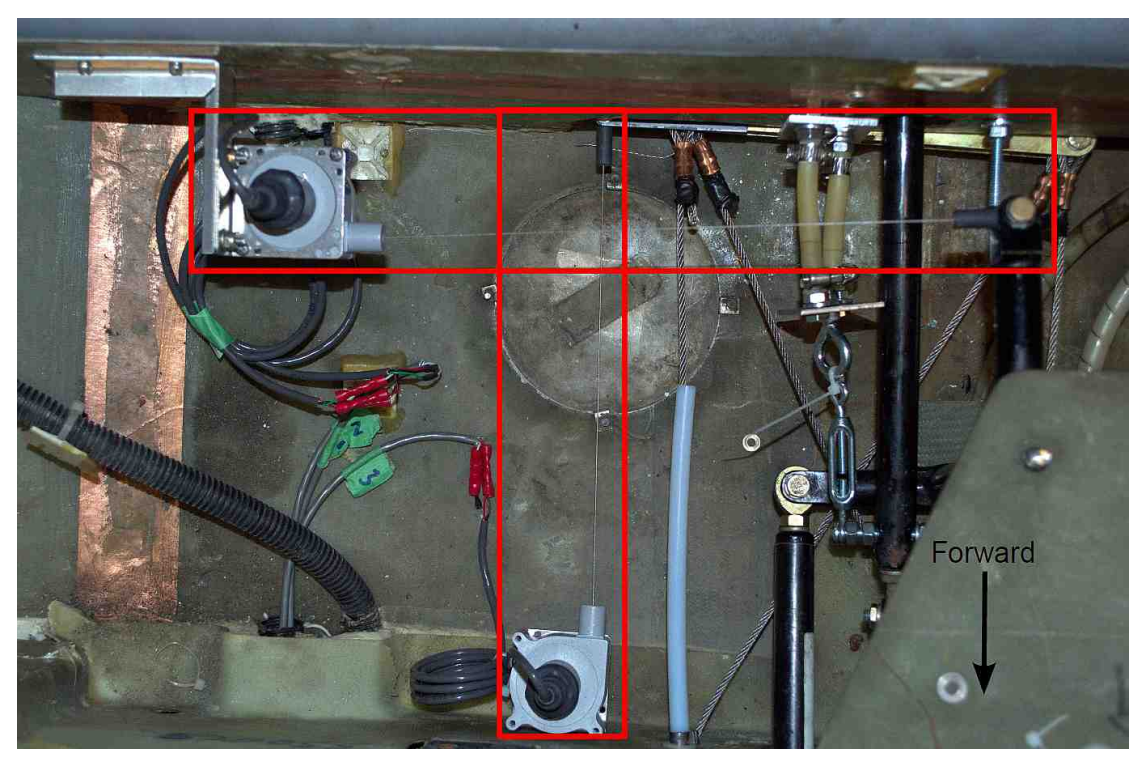

Figure 2.7: String potentiometers attached to modified control rods beneath the baggage compartment for measuring yaw and roll inputs.

signals from these string potentiometers are transmitted by way of wiring running to a custom electronics interface board mounted on the base of the support structure as will be described in Section 2.9.

\subsection{Instrument Panel}

To ensure the best possible physical fidelity, it was decided that as many of the original panel components as possible would be used in the KatanaSim. Each Katana is equipped with an aluminum instrument panel which provides the mounting points and structure to display the flight and engine gauges, avionics stack, circuit breakers, and various switches. The panel in each aircraft is instrumented slightly differently, with various combinations of avionics, flight gauges, and compass styles. Figure 2.9 shows one particular cockpit configuration with a half stack of avionics in the centre and only 7 of 8 possible flight gauges installed.

The majority of the panel components had to be replaced with simulated COTS com- 


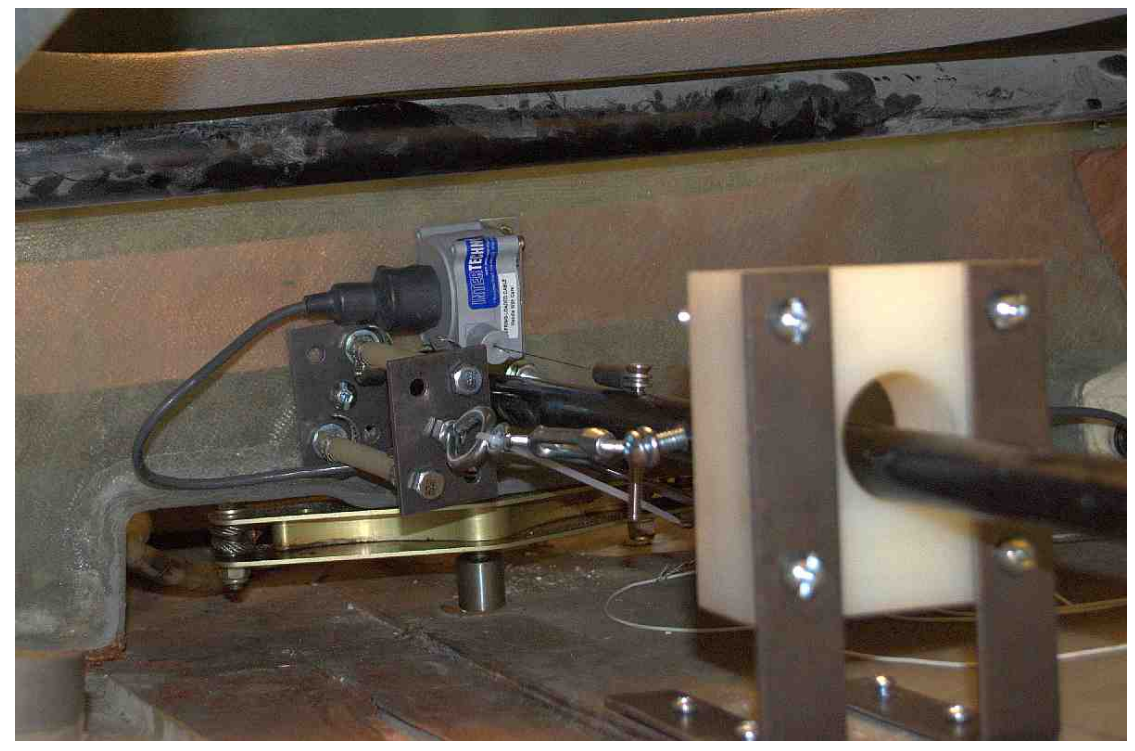

Figure 2.8: String potentiometer mounted to the aft side of the B-bulkhead for measuring pitch inputs.

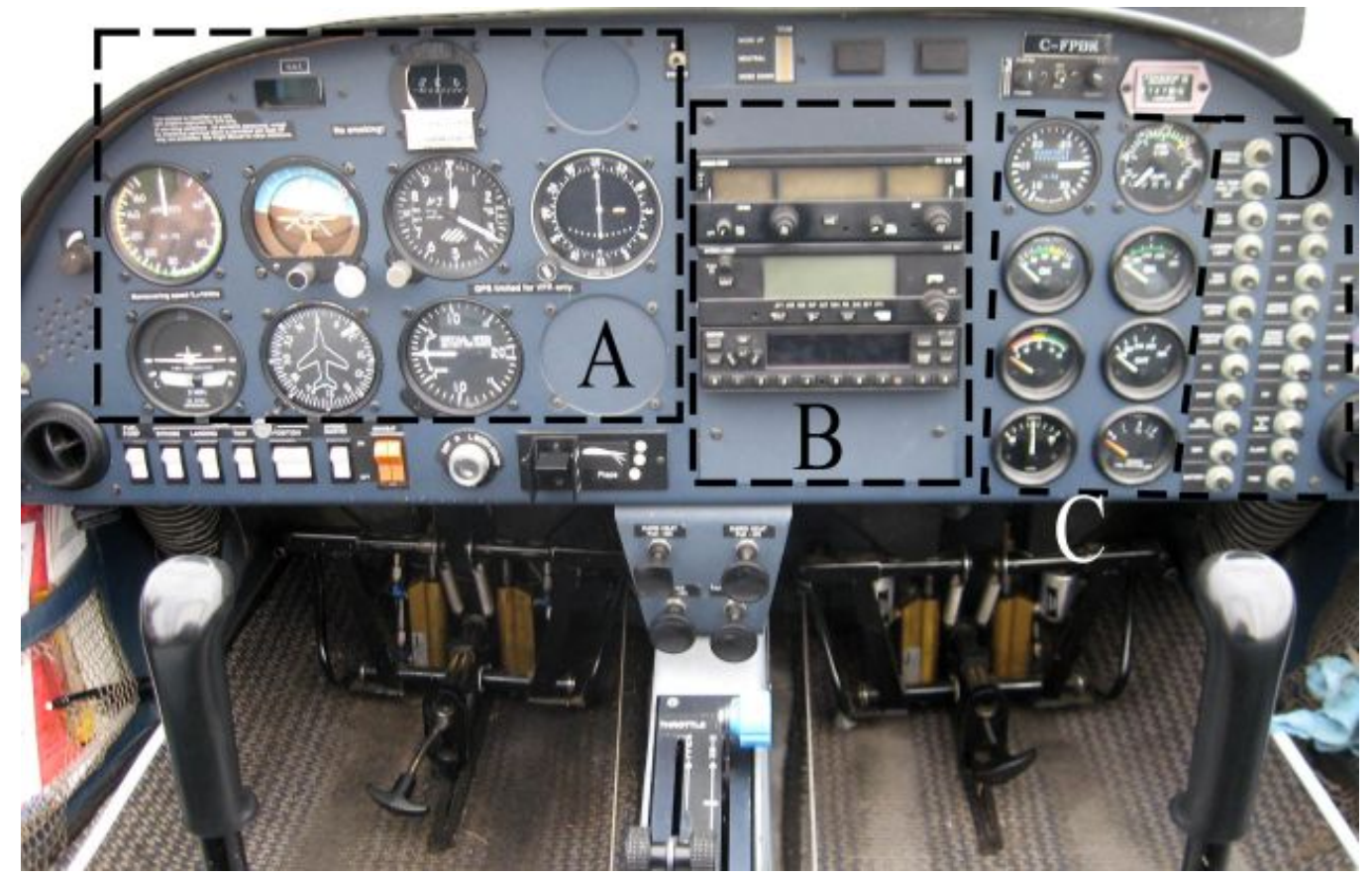

Figure 2.9: Instrument panel of a Katana at OAS showing location of flight instruments (Area A), avionics (Area B), engine instruments (Area C), and circuit breakers (Area D). 


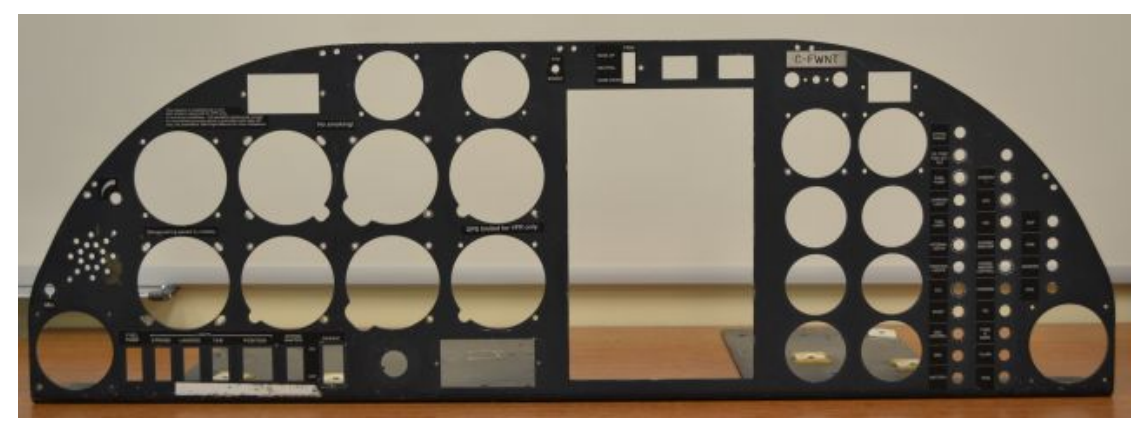

Figure 2.10: The original, unpopulated aluminum instrument panel.

ponents for reasons discussed in the following sections. Due to small differences in the geometry of the original and simulated parts, the original aluminum instrument panel, seen in Figure 2.10. could not be used. The simulated flight and engine gauges, as well as the circuit breakers, were all built with dimensions approximately one to two millimeters larger than the original parts. Enlarging the cutouts in the panel would have been difficult but also would have resulted in the cutouts overlapping as they were already arranged quite tightly. A new instrument panel for the KatanaSim was reverse engineered in Pro/Engineer and modified with dimensions tailored to fit the simulated instruments. The design was manufactured by Sentry Precision (Ottawa, Canada) from a sheet of $0.3175 \mathrm{~cm}$ (0.125 inch) thick steel. Steel was chosen over aluminum to minimize cost, recognizing that the minimal increase in weight would not be a concern for the simulator. The resulting unpopulated instrument panel was visually indistinguishable from the original aircraft part with the only exception being in the paint colour, which was chosen to be brown rather than the original blue-grey due to the colour availability of rust-proof paint.

\subsubsection{Flight Gauges}

A DA20-A1 Katana is typically equipped with a floating compass as well as eight flight gauges: airspeed indicator, artificial horizon, altimeter, very high frequency omnidirectional range indicator (VOR), turn and bank coordinator, heading indicator, vertical ve- 
locity indicator, and automatic direction finder (ADF). The airspeed indicator, altimeter, and vertical velocity indicator readings rely on air pressures from the pitot static system. The artificial horizon, turn and bank coordinator, and heading indicator gauges are gyro instruments. The VOR and ADF are navigational aids that receive and interpret radio signals from fixed navigation stations and the floating compass relies on the earth's magnetic field to maintain the correct orientation [19]. As such, if these nine instruments were to be installed in the KatanaSim, they would require several complex systems to be set up in order to stimulate the instruments and acquire the desired readings. It was decided that sourcing and purchasing simulated instruments was the best available option for populating the instrument panel.
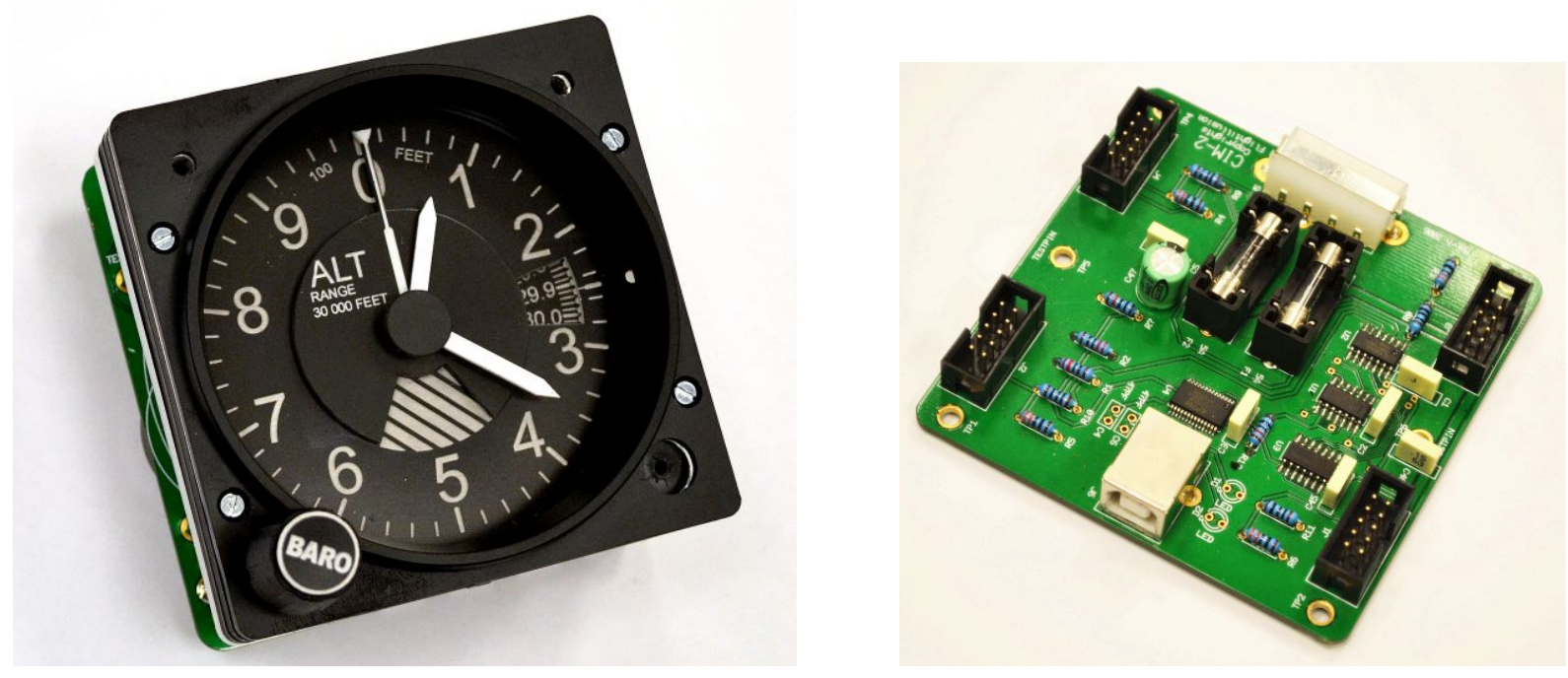

Figure 2.11: A Flight Illusion altimeter and central module board.

The simulated flight instruments and floating compass were purchased from Flight Illusion (Waver, Belgium) [20], a company specializing in aircraft simulator components. The instruments closely resemble those in a Katana, though the altimeter required the addition of a custom-made faceplate and the floating compass was mounted atop the dash panel rather than being installed with the other flight gauges. The instruments were connected via ribbon cable to a powered central module board (shown in Figure 2.11 
along with the altimeter purchased from Flight Illusion) which was then connected to the simulation computer via a USB cable.

The instrument panel with the final installation of flight instruments appears in Figure 2.12 .

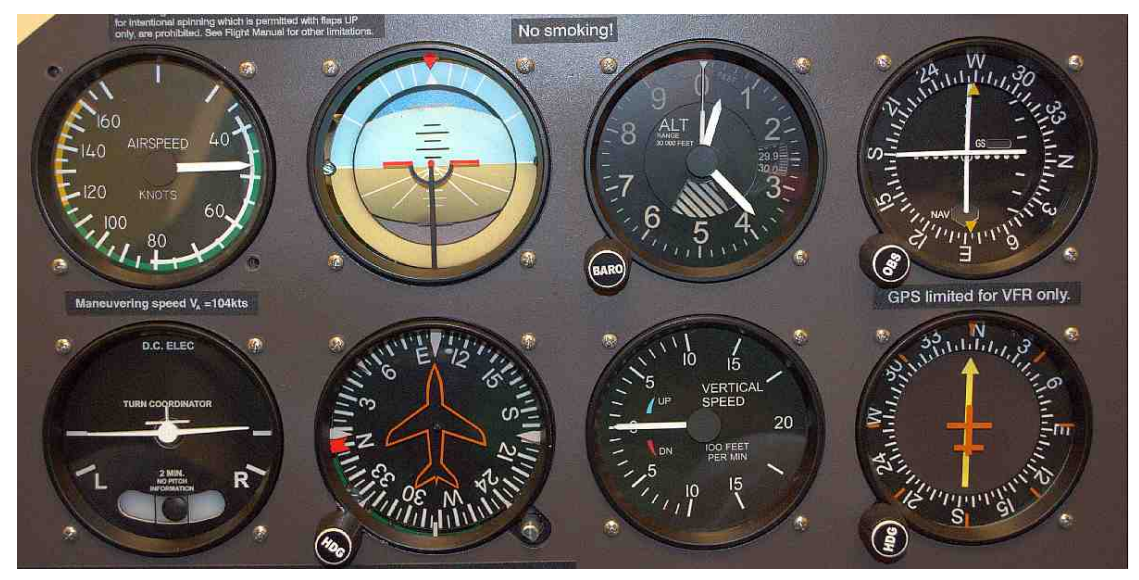

Figure 2.12: Flight Illusion flight instruments installed in the KatanaSim instrument panel. Top row from left: airspeed indicator, artificial horizon, altimeter, and VOR. Bottom row from left: turn and bank coordinator, heading indicator, vertical velocity indicator, and $\mathrm{ADF}$.

\subsubsection{Avionics}

The simulated avionics stack chosen to be installed in the KatanaSim consisted of an audio panel, two radios, an ADF receiver, a Distance Measuring Equipment (DME) receiver, and a transponder. These simulated avionics were also purchased from Flight Illusion. Figure 2.13 shows the Flight Illusion avionics stack after installation in the KatanaSim.

\subsubsection{Engine Gauges}

Another major group of instruments located on the right-hand side of the instrument panel of a typical Katana comprises the eight engine instruments: tachometer, manifold pressure gauge, oil pressure gauge, oil temperature gauge, ammeter, voltmeter, cylinder 


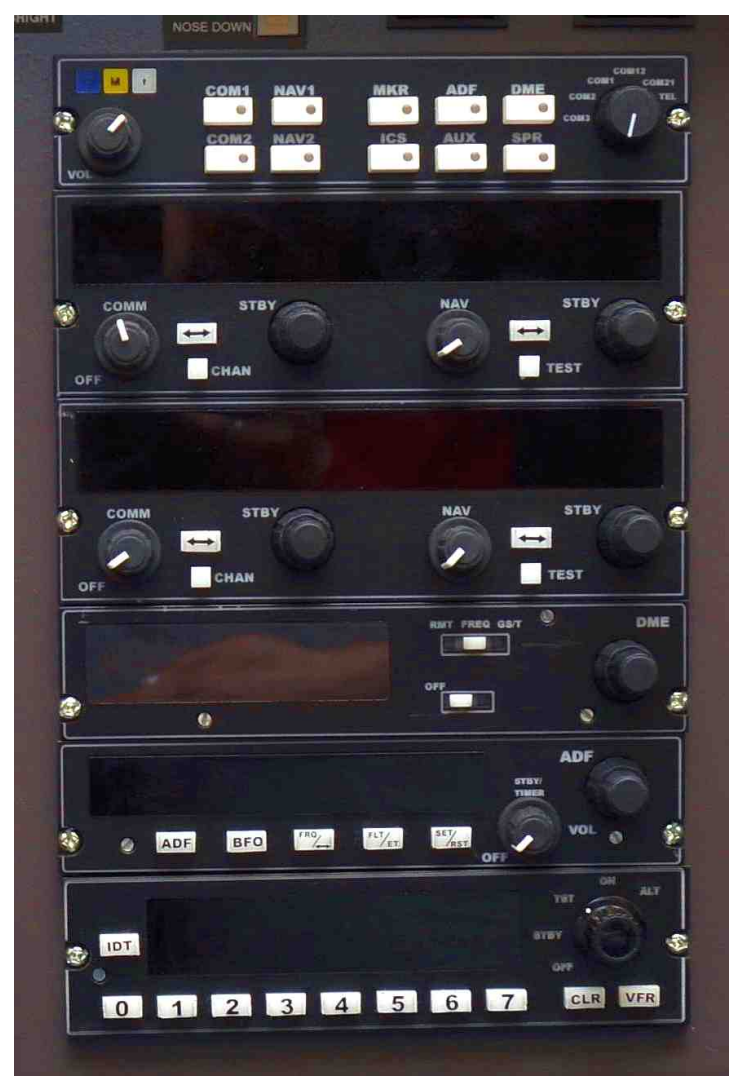

Figure 2.13: Flight Illusion avionics stack installed in the KatanaSim instrument panel. 


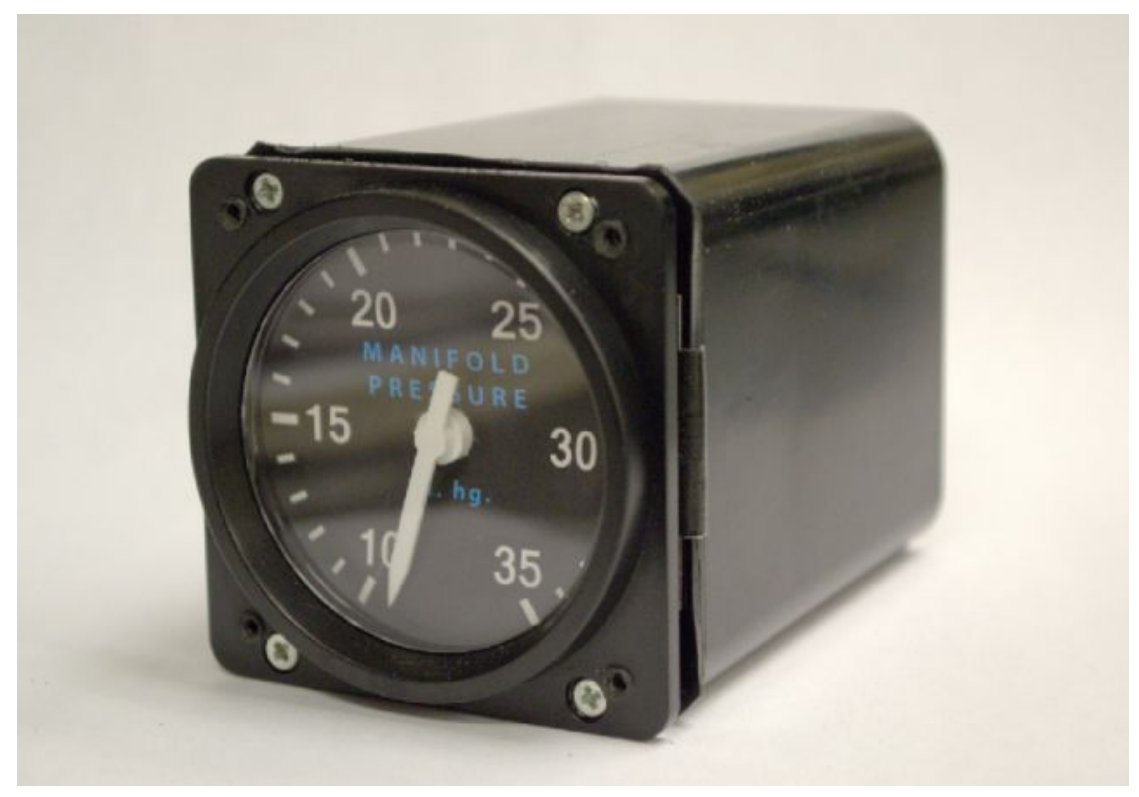

Figure 2.14: A SimKits aircraft gauge, customized to act and look like a manifold pressure gauge.

head temperature gauge, and the fuel gauge. These instruments receive data from the Katana's Rotax engine, generator, and fuel system. As with the flight gauges, it was decided that stimulating the aircraft gauges would be overly complex. Simulated instruments were purchased from SimKits (Giessenlanden, The Netherlands) [21], another supplier specializing in aircraft simulator components. The gauges from SimKits, unlike those from Flight Illusion, were not purchased in a ready-to-use configuration but instead were customizable gauges that required assembly as well as custom-designed face plates. These faceplates were recreated from photographs of the real aircraft gauges by Katherine Pyle ${ }^{\dagger}$ using Adobe PhotoShop. An example of a final assembled SimKits gauge can be seen in Figure 2.14.

While it would have been preferable to source all of the gauges from a single company, it was not possible to do so. Flight Illusion provided a superior product in that the flight gauges purchased were quieter and operated more smoothly than those from SimKits;

\footnotetext{
${ }^{\dagger}$ Intern architect, M.Arch 2011.
} 
however, engine gauges of the required size were not available from Flight Illusion.

The final installation of engine instruments appears in Figure 2.15.

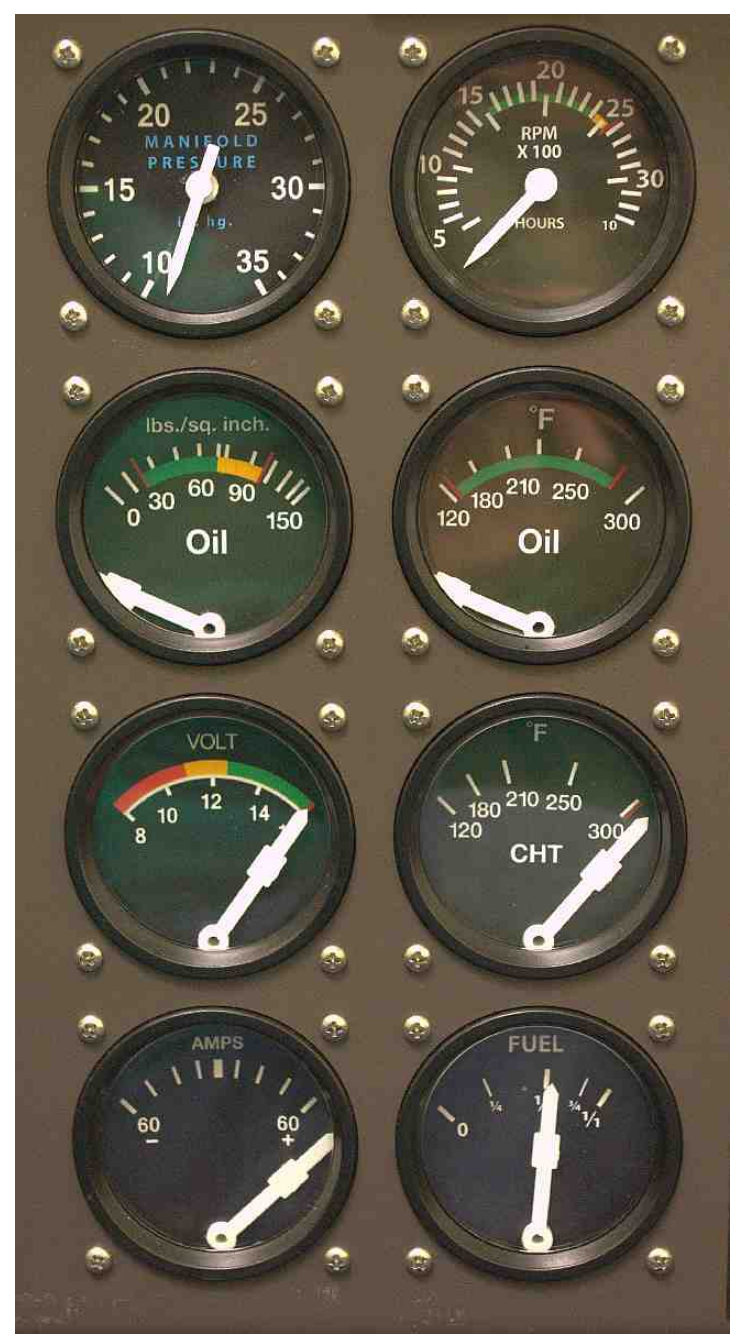

Figure 2.15: SimKits engine instruments installed in the KatanaSim instrument panel. Left column from top: manifold pressure, oil pressure, voltmeter, ammeter. Right column from top: tachometer, oil temperature, cylinder head temperature, fuel gauge.

\subsubsection{Switches and Circuit Breakers}

The instrument panel of a Katana can have as many as 27 circuit breakers, which are located on the far right side of the panel. As the aircraft flight circuit breakers require between 5 and 40 amps to trip, it was decided that simulated circuit breakers would be a 
safer option for use in the KatanaSim.

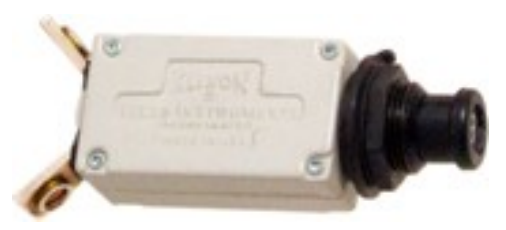

Figure 2.16: Klixon 3SB series simulator circuit breaker.

Twenty-seven Klixon 3SB series simulator circuit breakers, one of which is shown in Figure 2.16, were purchased from Sensata Technologies (Massachusetts, USA). These simulated circuit breakers trip at 200 milliamps and can be tripped remotely from the Instructor Operating Station (IOS) or manually by a simulator occupant. These were installed in the instrument panel as shown in Figure 2.17. Note that a GPS circuit breaker has not been installed as there is no GPS in the simulated avionics stack in the KatanaSim.

The original flap controller and trim switches from the aircraft were retained and installed in the instrument panel, with associated electronics modified for integration with the KatanaSim by Jonathan Plumpton ${ }^{\ddagger}$. The switches controlling the fuel pump, aircraft lights, generator, and battery were also installed without modification (see Figure 2.18), as were the original ignition switch and key. The wiring and software interface required for the switches and circuit breakers were implemented by Jonathan Plumpton [22].

\subsubsection{Completed Instrument Panel}

The populated KatanaSim instrument panel is shown in Figure 2.19. The placards were purchased through OAS Flight Centre direct from Diamond Industries. 


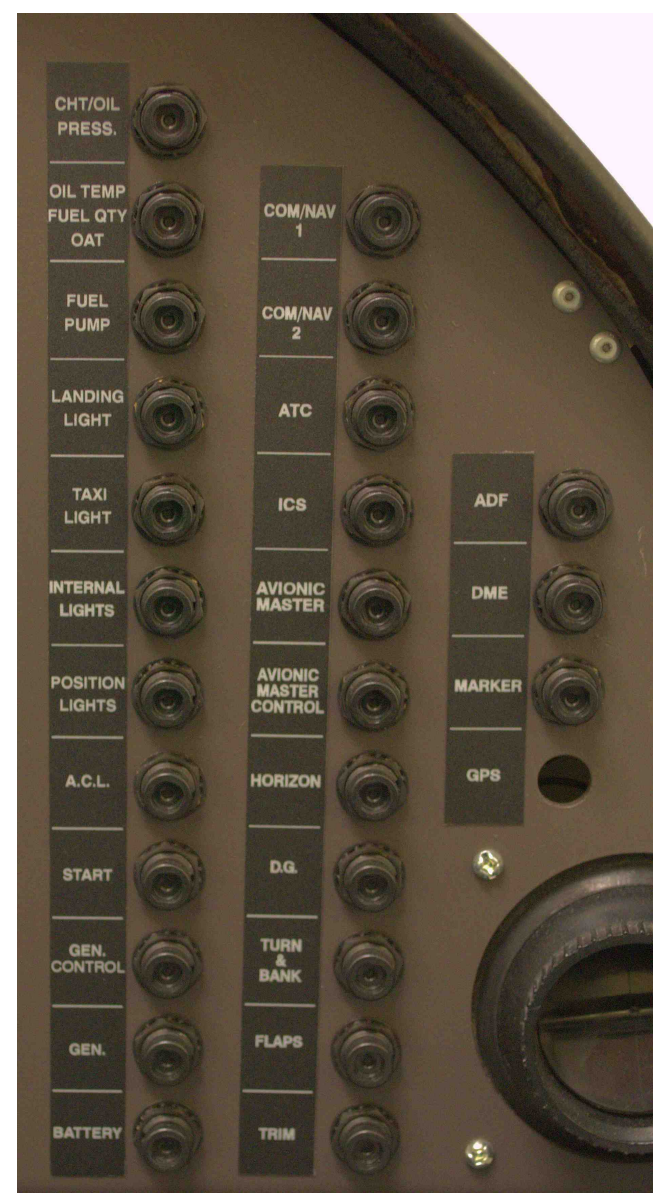

Figure 2.17: Simulated circuit breakers installed in the KatanaSim instrument panel.

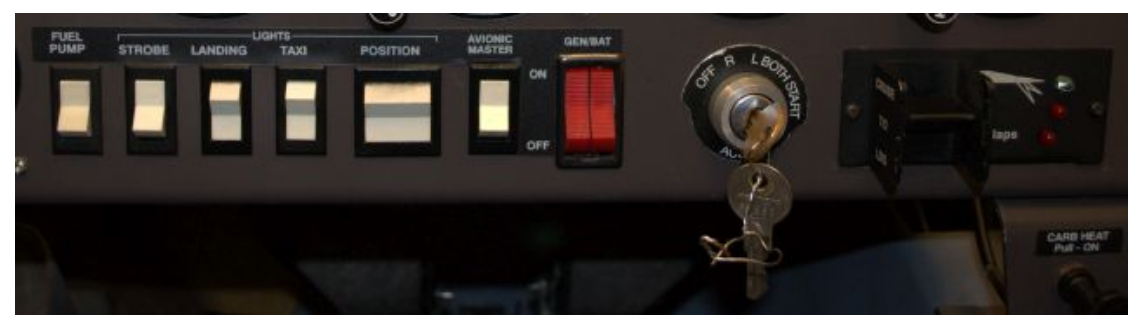

Figure 2.18: Original switches, ignition, and flap controller installed in KatanaSim instrument panel. 


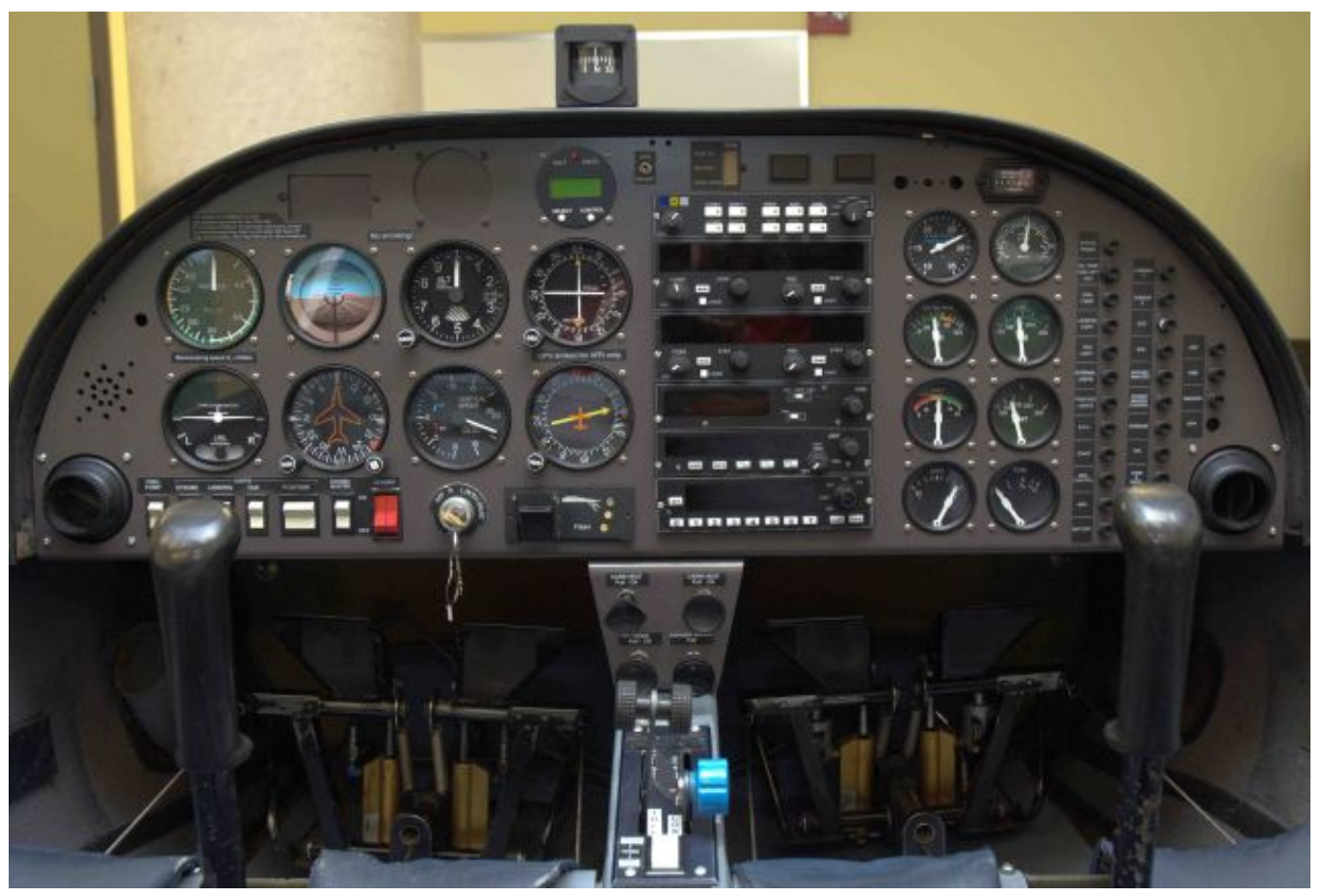

Figure 2.19: Populated KatanaSim instrument panel.

\subsection{Audio Cueing}

The Logitech Z523 Speaker System, as pictured in Figure 2.20, was chosen as it was deemed capable of providing the required sound effects, and was cost effective and readily available from local consumer electronics stores. The two speakers were bolted to a small shelf on the front side of the firewall, oriented to face the occupants but out of view. The subwoofer was secured beneath the fuselage on the base of the simulator support structure. The audio for the KatanaSim was provided by the software package discussed in Section 2.12.

\subsection{Visual Cueing}

Typical projection screens and computer monitors can prevent a simulator occupant from feeling truly immersed in their environment as they remain aware of their location in a

\footnotetext{
${ }^{\ddagger}$ M.A.Sc Candidate in the Applied Dynamics Laboratory at Carleton University.
} 


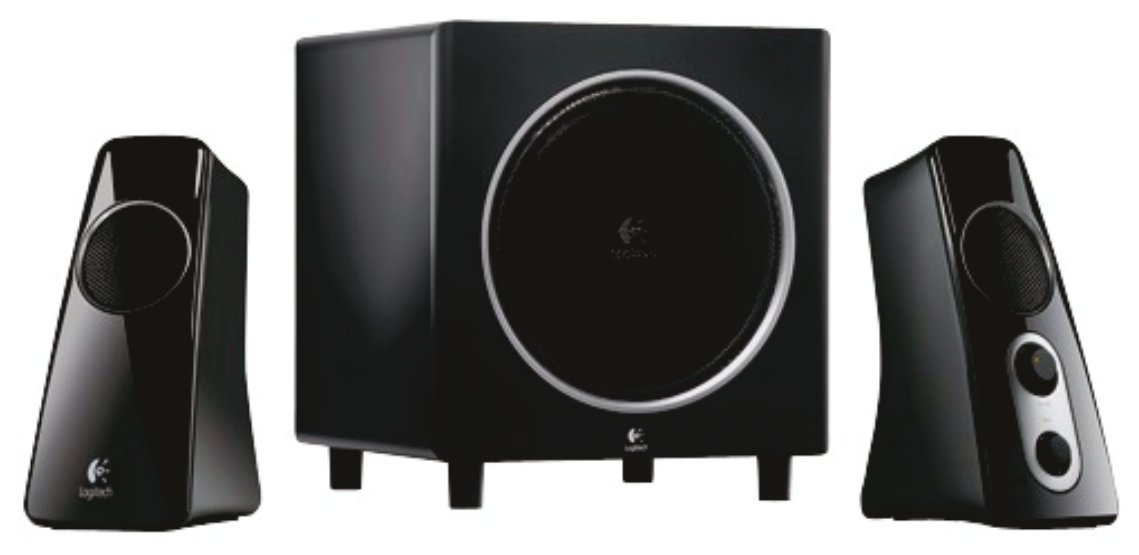

Figure 2.20: The Logitech Z523 speaker system [23].
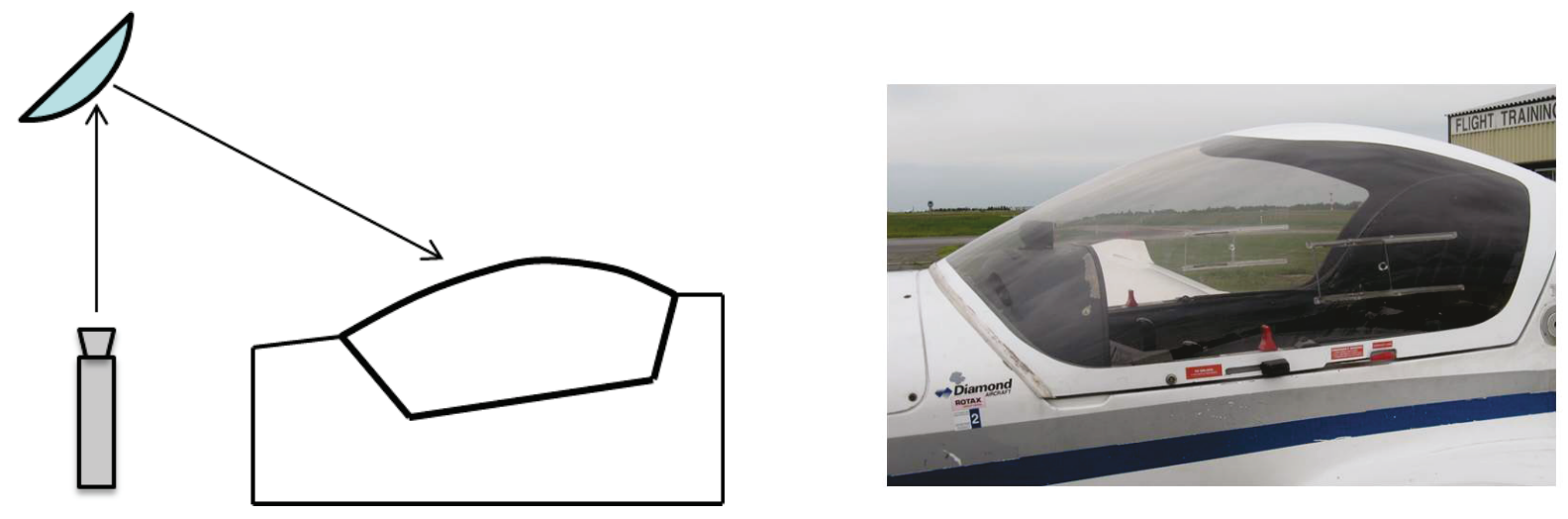

Figure 2.21: Immersive visual system concept sketch and the Katana canopy. 
classroom. A novel design for an immersive visual system was initially conceived using the original aircraft canopy; it would be given an opaque coating which would enable the visuals to be back-projected onto the canopy and viewed from within the cockpit. In order to create a projection for the irregular surface shape of the canopy, the image would be projected through a custom set of lenses which would appropriately distort the image. With the aircraft canopy closed and the visual system running, an occupant of the KatanaSim would be completely immersed in the training experience. This design was prepared in partnership with B-Con Engineering (Ottawa, Canada). However, due to logistical issues encountered by B-con, this visual system design was unable to be realised in the time frame of this project.

In lieu of the novel design, a simple interim visual system design was implemented to provide the visual cueing for the simulator occupants. This system was designed and implemented by Jonathan Plumpton and is shown in Figure 2.22. A projector screen was bolted to two triangular steel frames mounted to the front of the KatanaSim support structure. The Epson Powerlite Home Cinema 3010 projector was mounted to the top of the fuselage over the baggage compartment. This design lacks the immersive qualities of the previously-discussed design but still provides adequate visual cueing for training purposes.

\section{7 $\quad$ Brakes}

Steering a Katana during taxiing and ground rolls is accomplished using the brake pedals (located just above each rudder pedal) to control the disc brakes installed on the left and right main landing gear; the nose wheel is on a free caster and has no steering or braking ability. The brake system, shown in Figure 2.23, consists of two brake fluid reservoirs located on each of the co-pilot's rudder pedals, four master cylinders located on each of 


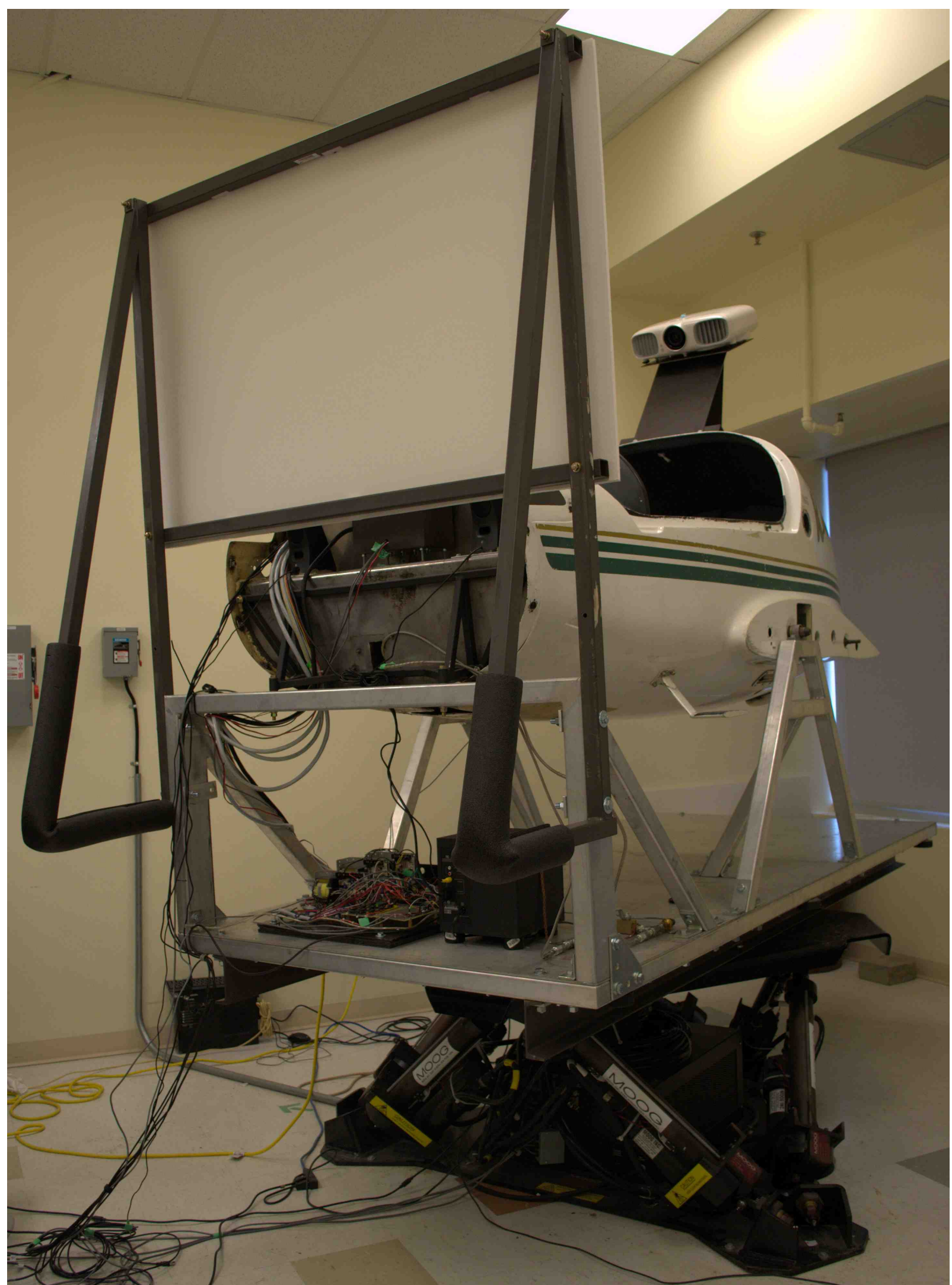

Figure 2.22: View of the KatanaSim showing the projection screen and mounting frame, as well as the projector mounted on the top of the fuselage. 
the pilot's and co-pilot's rudder pedals, the brake lines, parking brake valve, calipers, and brake discs.

The parking brake mechanism for the KatanaSim was designed completely separately from the left and right main brakes; it is described in Subsection 2.9.2. The KatanaSim main brakes use the same equipment setup as the Katana with the exception of the parking brake valve and disc brakes. The cylinders and reservoirs on the rudder pedals were installed normally and the two brake lines were guided through the interior of the fuselage and out through the front wall of the KatanaSim. The two brake lines, rather than being connected to a parking brake valve, were instead connected to two PX181B pressure sensors from Omega (Laval, Canada) [24]. Several COTS adapters and valves, and one custom-made adapter were used to make this connection as no standard plumbing components were compatible with the brake line connectors. The adapters and sensors were securely strapped to the front left corner of the base of the KatanaSim mount, as shown in Figure 2.24. The sensors were then wired to the electronics interface board where they were connected to a $5 \mathrm{~V}$ power source and an Arduino Mega 2560 microcontroller board [25] which collects the sensor outputs.

The lines were filled with brake fluid so that full pressure applied by a simulator occupant to a brake pedal would result in no more than $2.17 \mathrm{~cm}$ (0.5 inches) compression of the master cylinder located behind the panel; this mimics the brake feeling of a wellmaintained Katana as the brakes are usually quite stiff.

A tee junction was included between the brake lines and the pressure sensors and a valve was mounted to the open side of the tee. This not only provided a convenient access point for filling the lines but also provides the option for subsequently adding a brake failure system should one be required. A brake system failure in the Katana is generally in the form of a loss of pressure due to a leak or rupture in the system. A pilot tests the system in flight by applying pressure to the brake pedals; if the pedals depress too 


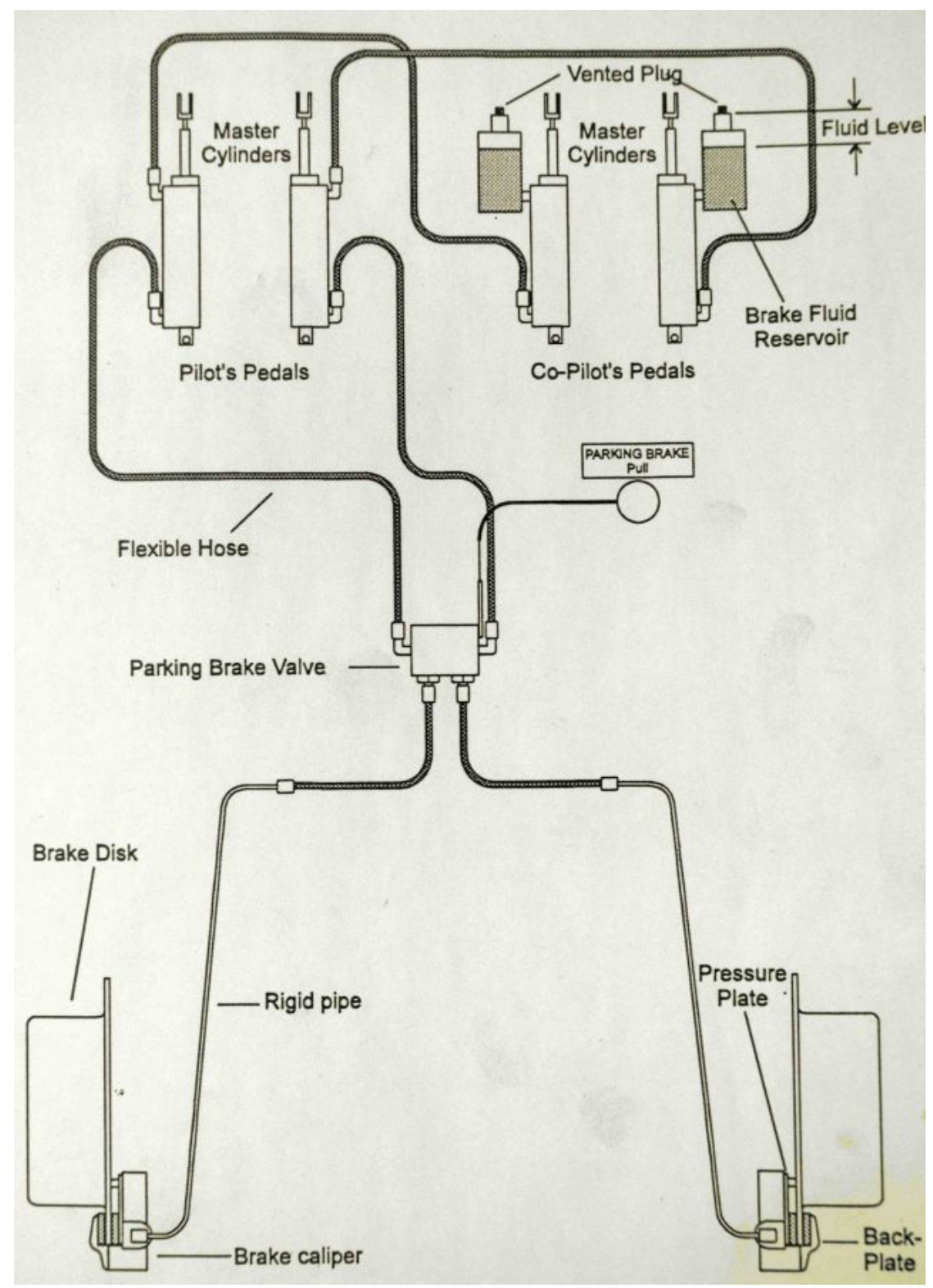

Figure 2.23: The brake system of a Katana [18]. The KatanaSim uses the reservoirs and master cylinders but the brake lines are attached to pressure sensors rather than the parking brake valve; the disc brakes are not installed on the KatanaSim. 


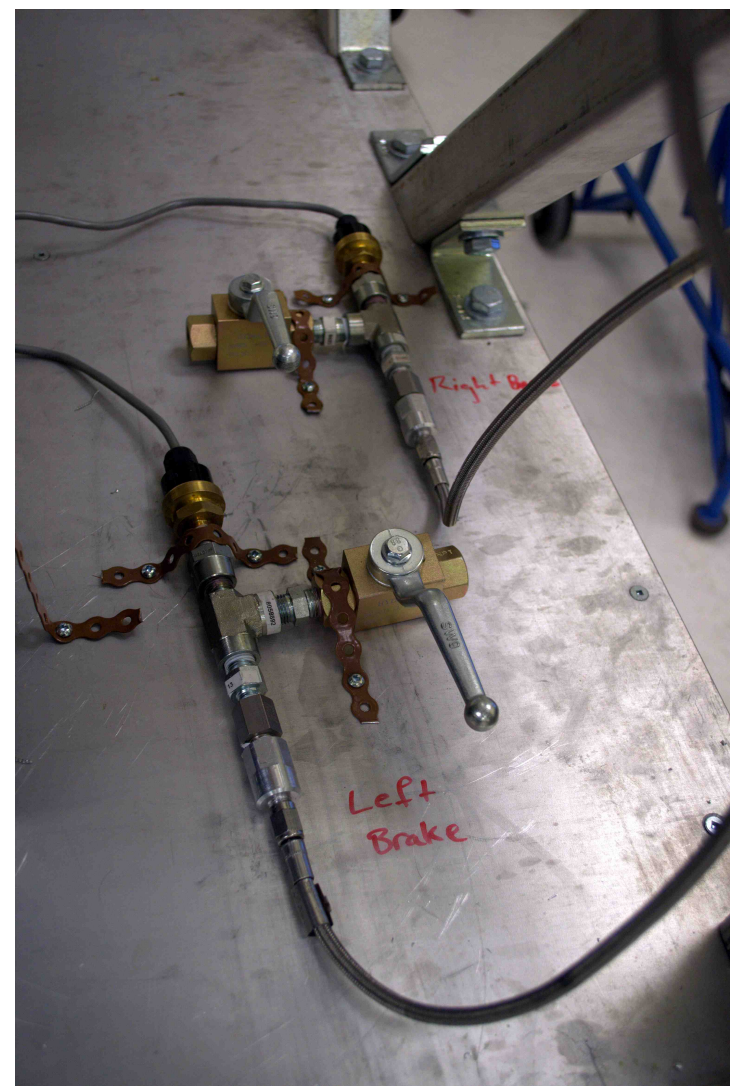

Figure 2.24: Brake lines, valves, and pressure sensors secured to the base of the KatanaSim. 
easily, the pilot must make plans to adjust the landing distance accordingly. The use of an accumulator was investigated as a method to drain and fill the lines while keeping the system closed and the brake fluid contained. An accumulator is a small tank which contains an adjustable gas-filled bladder which can be expanded or contracted in order to create a change in pressure inside the tank which then increases or decreases the fluid level in the system. An accumulator was found to be a promising option if a brake failure system was implemented in the future.

\subsection{Haptic Feedback}

The first haptic feedback system considered for installation in the KatanaSim was a set of three control loaders. Each control loader would be capable of providing force feedback through one of the three control systems (corresponding to roll, pitch, and yaw) to the simulator occupant. The control loaders would interface with the simulation software in order to provide active feedback, changing the feel of the flight controls for each flight condition encountered. The detailed design of this system was not completed as control loaders of the caliber suitable for installation in the KatanaSim cost $\$ 75000$ dollars (see quote from Servos \& Simulations Inc. in Appendix A). Due to the KatanaSim project objective of investigating options for producing a cost-effective simulator, it was agreed that a second, more cost-effective solution would be more appropriate. Active control loaders can be implemented in a subsequent phase of the project once the feasibility of the simulator has been proven since they are a low-risk, though high-cost, addition to the system. As an active haptic cueing system is required by Transport Canada for certification as a full flight simulator (a long-term goal of the KatanaSim project), this system will need to be implemented before the certification process can begin.

An interim solution was adopted in which compact adjustable spring assemblies were 
used to provide feedback to the control column. Alterations to the rudder pedals were deemed unnecessary as springs built in to the pedal system provided resistance to inputs and centred the pedals when no other inputs were present.

\subsubsection{Requirements}

The interim haptic feedback system was not able to respond actively to inputs as actuated control loaders would, so a single spring constant for each degree of freedom had to be selected. In different manoeuvres flown in the aircraft (e.g., take off roll, slow flight, cruise, etc.), a pilot will require different amounts of force to create the same roll and pitch responses because of the changes in air pressure over the control surfaces. Greater airspeed leads to greater forces required to deflect the control surfaces. As many of the manoeuvres in a typical training flight start from the aircraft at cruise, it was decided that the haptic feedback should mimic the feeling of the controls in that flight condition. In order to accomplish this, Dan Cockerham, a flight instructor at OAS Flight Centre, used a hand-held scale to measure the forces on the control column required to produce various degrees of roll and pitch during flight. The flight conditions during the test measurements were as follows: $12^{\circ} \mathrm{C}$ outside air temperature, $610 \mathrm{~m}(2000 \mathrm{ft})$ above sea level, cruise configuration, $185 \mathrm{kph}$ (100 knots) indicated airspeed, $81 \mathrm{kPa}$ (25 inches of mercury) manifold pressure, and 2300 engine RPM. The recorded forces are listed in Table 2.8.1.

Neither the aggressive nor the gentle roll and pitch inputs required full deflection. It was decided that the force required for full deflection for pitch and roll should be approximately $12.0 \mathrm{~N}$.

\subsubsection{Design and Installation}

A simple mechanical design using bolts, small steel plates, surgical tubing, and turn buckles was used to create the adjustable spring system. Five $0.635 \mathrm{~cm}(0.25 \mathrm{in})$ holes were drilled 
Table 2.1: Forces required by the pilot on the control column handle to achieve gentle and aggressive roll and pitch angles.

\begin{tabular}{lc}
\hline Manoeuvre & Force required on control column handle \\
\hline Gentle pitch up, $10^{\circ} / 3 \mathrm{sec}$ & $4.9 \mathrm{~N}$ \\
Gentle pitch down, $10^{\circ} / 3 \mathrm{sec}$ & $3.9 \mathrm{~N}$ \\
Aggressive pitch up, $10^{\circ} / 1 \mathrm{sec}$ & $10.8 \mathrm{~N}$ \\
Aggressive pitch down, $10^{\circ} / 1 \mathrm{sec}$ & $9.8 \mathrm{~N}$ \\
Gentle roll right, $10^{\circ} / 3 \mathrm{sec}$ & $2.9 \mathrm{~N}$ \\
Gentle roll left, $10^{\circ} / 3 \mathrm{sec}$ & $2.9 \mathrm{~N}$ \\
Aggressive roll right, $10^{\circ} / 1 \mathrm{sec}$ & $11.7 \mathrm{~N}$ \\
Aggressive roll left, $10^{\circ} / 1 \mathrm{sec}$ & $11.7 \mathrm{~N}$ \\
\hline
\end{tabular}

in each of the two steel plates, which were each $3.8 \mathrm{~cm} \times 5.08 \mathrm{~cm}(1.5$ in $\times 2$ in). Eye hooks were secured in the middle hole of each with washers, lock washers, and nuts. In two of the remaining holes, diagonally opposed from each other, two $0.635 \mathrm{~cm}(0.25 \mathrm{in})$ bolts were installed and secured. A small length of rubber tubing was then placed over the end of each of the two bolts on the plate with the other ends of each tube placed over the ends of the bolts of another plate-and-bolt subassembly. The tubing was secured to these bolts using hose clamps; and a turn buckle was connected to one of the eye bolts. A photograph of one of these assemblies can be seen in Figure 2.25.

Each adjustable spring assembly was able to be tuned using coarse and fine methods. Coarse tuning could be performed by simply replacing the rubber tube lengths by shorter or longer lengths to increase or decrease the spring constant as desired. Fine tuning could be accomplished by tightening or lengthening the turnbuckle after installation.

Left pitch, right pitch, left roll, and right roll each required one of these assemblies to provide resistance to pilot inputs and to keep the control column centred when released. The two assemblies for roll inputs were installed underneath the seats of the simulator and connected to a rocker crank that is part of the control rod assembly. This installation corresponds to the one shown in Figure 2.25.

The subassemblies for the pitch system could not be installed in the same place as 


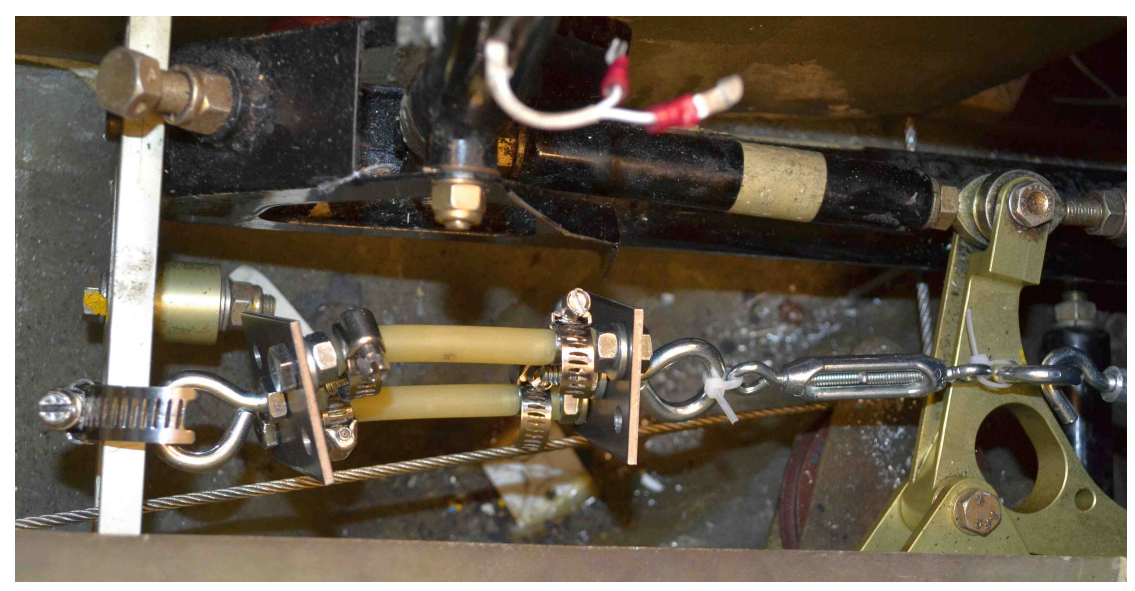

Figure 2.25: Half of the adjustable haptic feedback system for roll inputs found underneath the KatanaSim seats at the base of the control columns.

those for the roll system due to space constraints. The pitch subassemblies were instead installed in the rear portion of the simulator fuselage with the string potentiometers that measure control rod deflection. The two subassemblies were installed and connected to the pitch control rod. One of these subassemblies is shown in Figure 2.26.

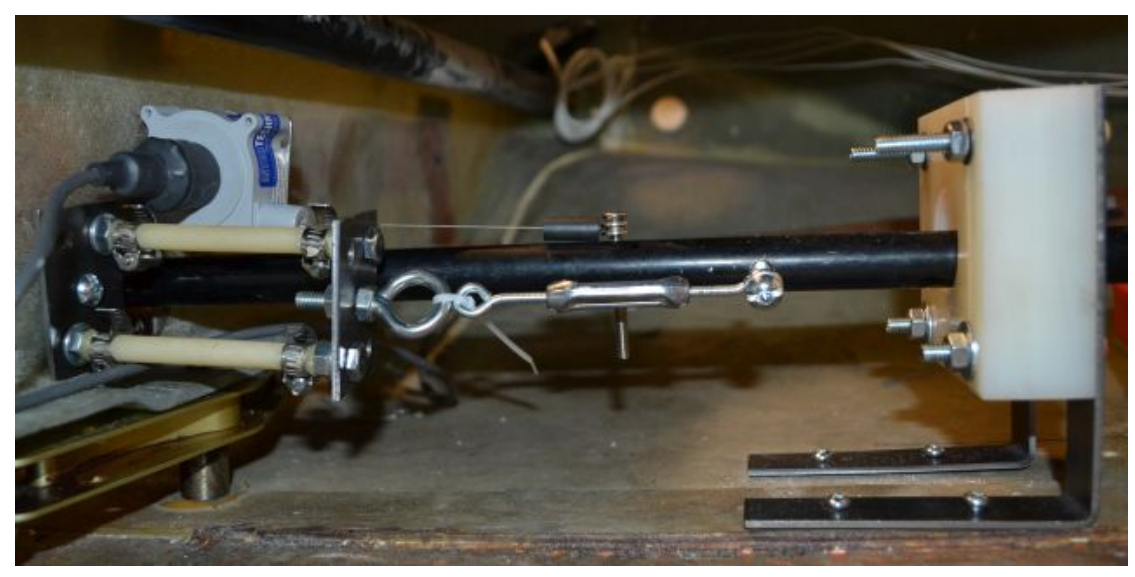

Figure 2.26: Half of the adjustable haptic feedback for pitch inputs located on the aft side of the B-bulkhead and attached to the elevator control rod; the other half of the system is located on the forward side of the bulkhead.

After installation, the turn buckles were used to fine tune the resistance of the control column to provide $11 \mathrm{~N}$ of resistance at full deflection as well as to ensure the stick returned to a neutral position when no pilot inputs were applied. 


\subsection{Electrical Components}

An electronics interface board, shown in Figure 2.27, was designed and built by Fred Barrett ${ }^{\S}$ to meet the electrical needs of the KatanaSim, providing power and transmitting signals for the various electrical components. This board sits on the base of the KatanaSim mount, and hosts three power sources $(5 \mathrm{~V}, 12 \mathrm{~V}, 24 \mathrm{~V})$ to power all of the simulator components with the exception of the visual system, audio system, computers, and motion platform. On the board there are also two Arduino Mega 2560 microcontroller boards which serve to send and receive signals communicated between the KatanaSim computers and the simulator components.

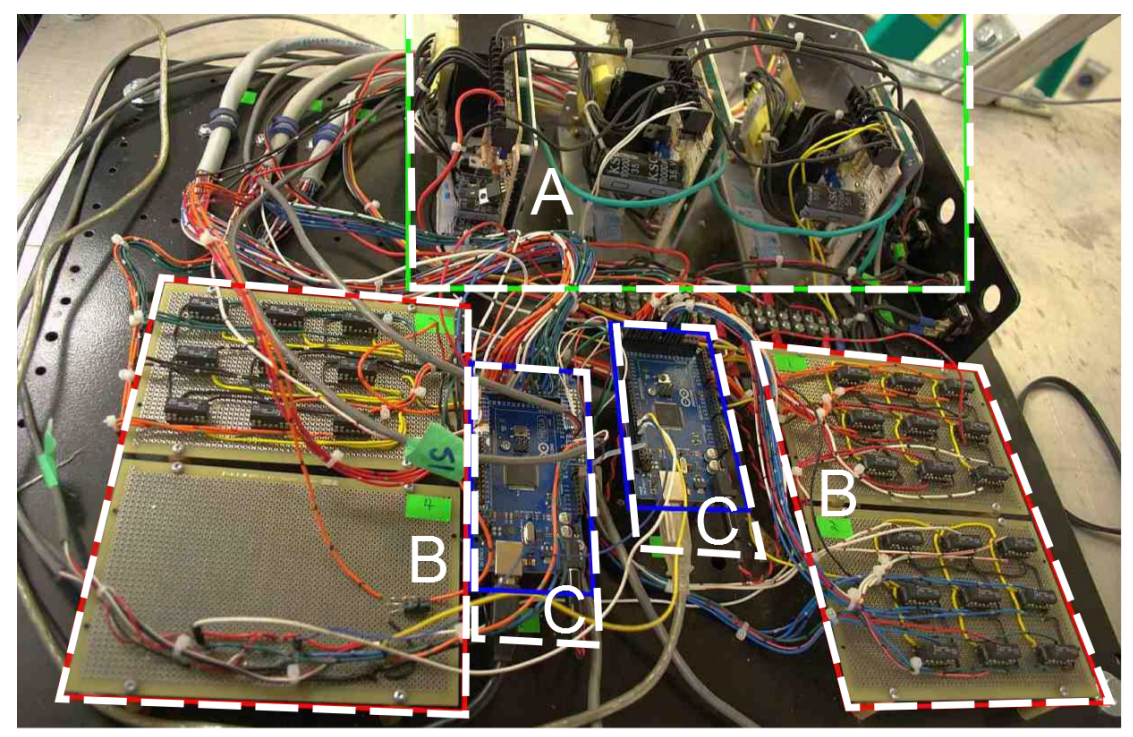

Figure 2.27: Electrical setup on the base of the KatanaSim: two Arduino Mega 2560 microcontrollers (Areas C), three power sources (Area B), and multiple reed relays and voltage dividers (Areas A).

\footnotetext{
${ }^{\S}$ An electrical engineer who supported electrical and electronic aspects of the project.
} 


\subsubsection{Instrument Panel Components}

All components installed on the KatanaSim instrument panel (i.e., flight and engine gauges, avionics, circuit breakers, and assorted switches) require both power and a method of transmitting and receiving signals to and from the simulator computers. The wiring for all of these components runs forward through a hole in the front firewall of the aircraft, down to the board on the base of the KatanaSim mount, and connects to the Arduino boards. Reed relays were required by the Arduino boards as the voltages required for the components was much higher than what could be provided through the Arduino boards. Additional information on the implementation of these electrical components can be found in Jonathan Plumpton's M.A.Sc thesis [22].

\subsubsection{Centre Console, Propeller Pitch, and Throttle Levers}

The centre console of a Katana, shown in Figure 2.28, has four knobs. These knobs control the choke, parking brake, cabin heat, and carburetor heat, respectively. Just below and aft of the centre console are located the two levers which control throttle and propeller pitch. In an aircraft, these knobs and levers have stiff cables running forward around the engine of the aircraft that push and pull the appropriate components as the knobs and levers are actuated. These cables were reused in the KatanaSim with the five cables being fed through the front firewall of the aircraft beyond which the cables were cut about six inches forward of the firewall. On the front side of the firewall is a small shelf, the same shelf that holds the speaker system, on which a custom-made steel enclosure was mounted (see Figure 2.29). Five rotary potentiometers were installed in this enclosure, each built with an arm $7.6 \mathrm{~cm}$ (3 inches) long. The cables from the knobs and levers were fed into the enclosure and each was hooked to a potentiometer arm. As the knobs and levers are pulled and pushed, the potentiometer arms are rotated accordingly. The wiring for these 


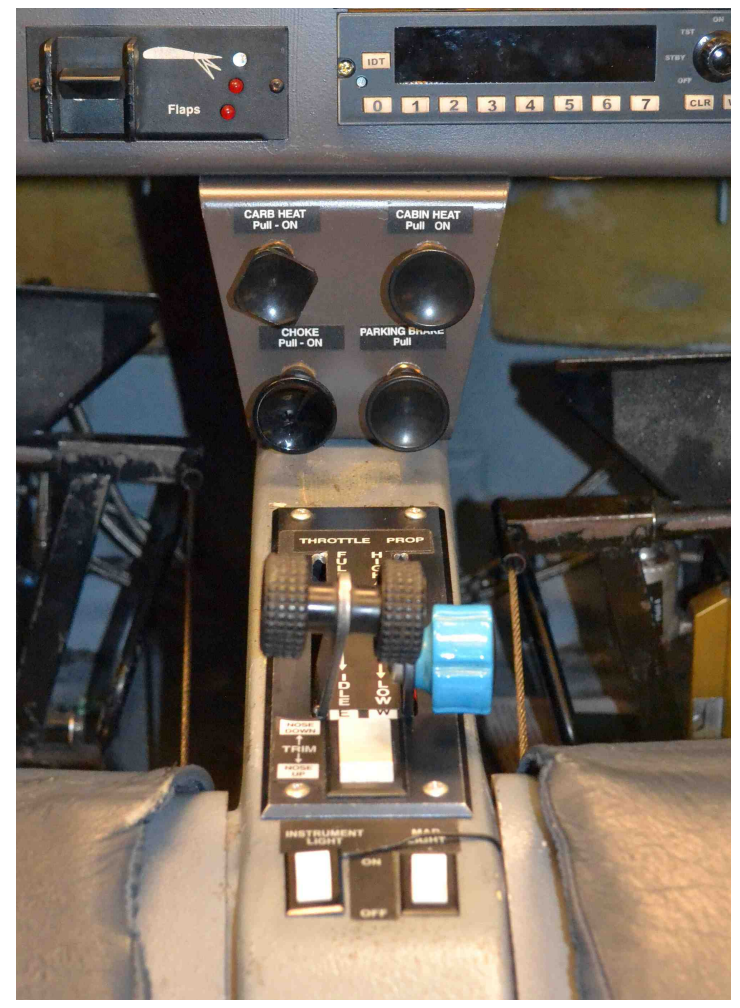

Figure 2.28: Centre console with pull knobs for carburetor heat, cabin heat, choke, and parking brake; below are the propeller pitch and throttle levers.

potentiometers runs through a hole near the base of the steel enclosure and down to the electronics interface board below.

\subsection{Computer Architecture}

The KatanaSim has four networked computers which communicate via user datagram protocol (UDP), with each assigned a specific and unique task: main, accessories, visuals, and instructor operating station (IOS). The main simulation computer runs the simulator software, communicates with all three other computers, and receives information from the flight controls, switches, and circuit breakers. The accessories computer provides additional processing power to lighten the computational load on the main computer by communicating with the gauges and avionics. The visual cueing system is run from a third 


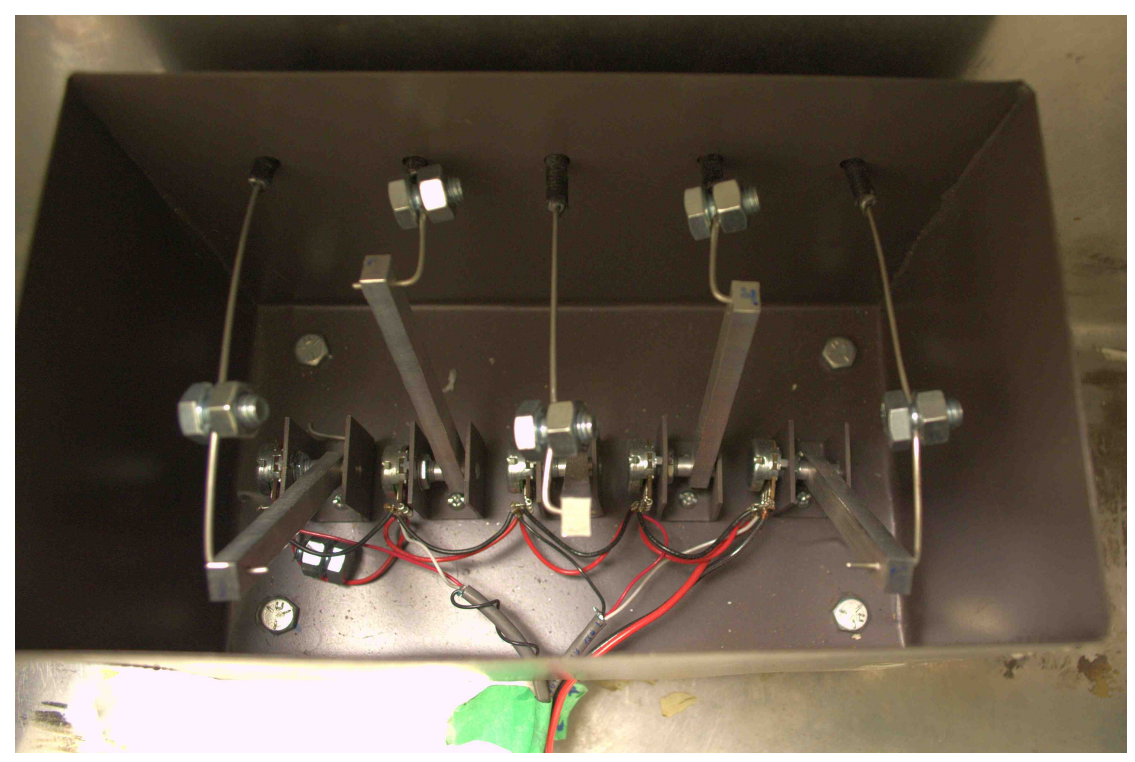

Figure 2.29: Top view of the potentiometer box on front shelf of engine firewall.

computer. The fourth and final computer is used as an IOS to give the instructor complete control over weather phenomena and flight conditions in the virtual environment, as well as the ability to cause equipment failures such as faulty gauges or tripped circuit breakers. The computer architecture (represented by the diagram in Figure 2.30) for the KatanaSim was developed by Jonathan Plumpton and is detailed in his M.A.Sc thesis [22].

\subsection{Motion Cueing}

The effect of a motion cueing system on the training effectiveness of flight simulators is an open research topic which goes beyond the scope of this thesis. The long-term goal of obtaining certification from Transport Canada as a Flight Training Device would not require the motion platform; however, the motion cueing system was include in the design and testing of the KatanaSim to evaluate the potential benefits and feasibility of certification as a full-flight simulator.

The KatanaSim was mounted on a MOOG 6DOF 2000E motion base located in the 


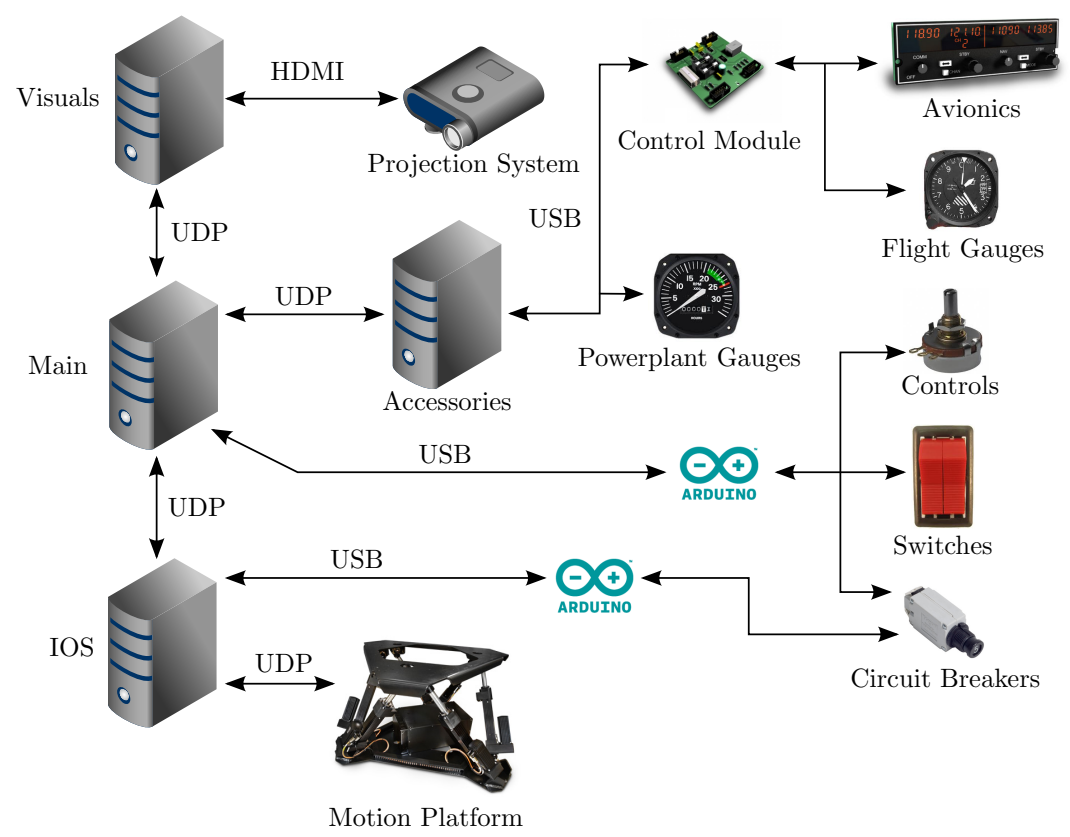

Figure 2.30: Layout of the KatanaSim computer architecture and electrical components.

Carleton University Applied Dynamics Laboratory. Figure 2.31 shows the motion platform before the simulator was mounted, and Table 2.2 provides the specific kinematic capabilities of the platform. The motion base was tuned for optimal use of the available motion envelope and provides both motion onset cues and sustained accelerations through the use of tilt coordination [10]. The tuning of the washout filter was done by Jonathan Plumpton and is discussed extensively in his M.A.Sc thesis [22].

Table 2.2: Kinematic capabilities of MOOG 6DOF 2000E motion base [10].

\begin{tabular}{lccc}
\hline Axis & Displacement & Velocity & Acceleration \\
\hline Surge & $\pm 0.25 \mathrm{~m}$ & $\pm 0.50 \mathrm{~m} / \mathrm{s}$ & $\pm 6.0 \mathrm{~m} / \mathrm{s}^{2}$ \\
Sway & $\pm 0.25 \mathrm{~m}$ & $\pm 0.50 \mathrm{~m} / \mathrm{s}$ & $\pm 6.0 \mathrm{~m} / \mathrm{s}^{2}$ \\
Heave & $\pm 0.18 \mathrm{~m}$ & $\pm 0.30 \mathrm{~m} / \mathrm{s}$ & $\pm 5.0 \mathrm{~m} / \mathrm{s}^{2}$ \\
Roll & $\pm 21.0 \mathrm{deg}$ & $\pm 30.0 \mathrm{deg} / \mathrm{s}$ & $\pm 500 \mathrm{deg} / \mathrm{s}^{2}$ \\
Pitch & $\pm 22.0 \mathrm{deg}$ & $\pm 30.0 \mathrm{deg} / \mathrm{s}$ & $\pm 500 \mathrm{deg} / \mathrm{s}^{2}$ \\
Yaw & $\pm 22.0 \mathrm{deg}$ & $\pm 40.0 \mathrm{deg} / \mathrm{s}$ & $\pm 400 \mathrm{deg} / \mathrm{s}^{2}$ \\
\hline
\end{tabular}




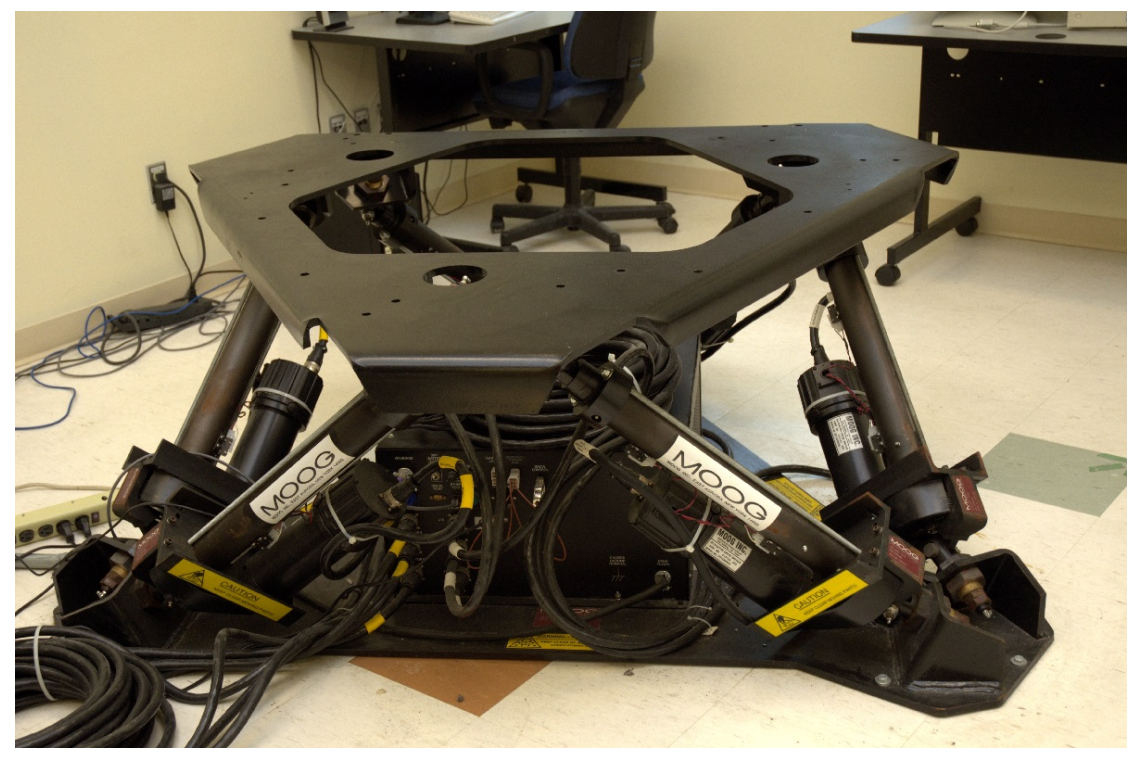

Figure 2.31: MOOG 6DOF 2000E motion base located in the Applied Dynamics Laboratory at Carleton University, used to provide motion cueing for the KatanaSim.

\subsection{Software Selection}

In a continuing endeavour to use as many COTS components as possible, it was decided that the flight simulation software environment would be chosen from those software packages which were commercially available. The two leading software packages on the market are Microsoft Flight Simulator (MSFS) [26] and X-Plane by Laminar Research [27]. It was decided that X-Plane was more compatible with the project objectives as it is highly customizable and has a reputation for excellent support for software and flight model development initiatives. Software plug-ins are a necessity for the KatanaSim project in order to integrate all of the hardware components and these plug-ins would be exceedingly difficult to implement with MSFS in comparison to X-Plane.

One of the largest differences between the two software packages is the flight model engine in each. X-Plane uses blade element theory (BET) rather than stability derivatives (used in MSFS) to calculate the flight model. A description and comparison of BET and stability derivatives will be discussed in detail in later chapters, but it can be said that 
the use of BET leads to more realistic aircraft dynamics in edge-of-envelope manoeuvres such as stalls and spins. BET can also be used to predict the flight dynamics of any given aircraft that a user can design [28]. In contrast, stability derivatives rely highly on predetermined handling characteristics for an aircraft. In X-Plane, a user can design an aircraft and see how that aircraft performs; in MSFS, a user can design an aircraft but must also specify the behaviour of that aircraft.

An additional advantage of using X-Plane was the knowledge that it has been used, on numerous occasions, in flight training devices and full flight simulators certified by the FAA [27]. As a long-term goal for the KatanaSim is to achieve certification by Transport Canada (which maintains standards nearly identical to those of the FAA), this was an important consideration.

\subsection{Budget}

One major consideration throughout the design process for the KatanaSim was cost effectiveness. Custom-built parts were avoided wherever possible and COTS components and original aircraft parts were used wherever possible. Funding was secured through industry partners (OAS Flight Centre and B-Con Engineering); grants from the Ontario Centres of Excellence and Mitacs Accelerate funding programs; and supplemented by funds from the Applied Dynamics Lab. The total cost of the KatanaSim development can be broken down into salary, hardware and manufacturing costs, and in-kind contributions from industry partners. The total cost of the project was $\$ 119800$ with salary accounting for $\$ 50600$, hardware costs accounting for $\$ 49900$, and in-kind contributions valued at $\$ 19300$. The cost of the motion platform $(\$ 67000)$ was not included in this budget as it is not intended to be a part of the final design. The KatanaSim was mounted on the platform for research purposes, but the long term goal of certification by Transport Canada as a Flight Training 
Device precludes the use of the motion platform.

This budget is considerably lower than the seven figure price tags associated with commercial airline flight simulators, and just under a quarter of the price of the King Air FTD currently used for training ab-initio students at OAS Flight Centre. It can be noted that the salary portion of the budget paid for the work done primarily by two graduate students over the course of two years. If a second KatanaSim were to be built, the salary cost would perhaps be increased if student wages were not in effect, but could be balanced by the much faster manufacturing time as the design phase would not be repeated and long lead times on purchases could be properly anticipated.

\subsection{Discussion}

Based on an analysis of the factors affecting training effectiveness discussed in Section 1.3.2, the KatanaSim has great potential to offer highly effective training to ab-initio student pilots. Of the five factors discussed, the "Difficulty of Task" is not discussed here as the difficulty of the flight manoeuvre is not affected by the simulator design.

Factors affecting the training effectiveness ratio of a simulator:

1. Visual fidelity: The quality of graphics and a well-developed virtual landscape affect training effectiveness. X-Plane's virtual environment includes basic landmarks such as highways, bodies of water, and basic representations of city infrastructure where appropriate. The virtual environment is customizable in case a specific landmark is desired, for example a building used as a reference point during circuit training.

The visual system hardware does leave room for improvement; a more immersive visual system, particularly one with side window views for the pilot, would be a good improvement, and one which would be necessary in order to achieve certification through Transport Canada. The current system is not believed to pose a risk of 
negative training transfer and is considered only to be an interim solution.

2. Procedural similarity: The high level of physical fidelity of the KatanaSim allows for excellent procedural similarity so students can gain familiarity with the aircraft layout and develop the muscle memory for tasks performed in the aircraft. X-Plane also has the ability to generate random local air traffic so students may practice their situational awareness and become accustomed to flying in busy airspace. X-Plane does provide the option of limited air traffic control interactions so that the student can listen to the Automatic Terminal Information Service station for information on weather and active runways, and contact Clearance, Ground, and Tower control frequencies to request and receive clearance for taxi and take-off. As radio communications are considered by many students as one of the more stressful aspects of flight training, even these basic provisions in X-Plane can provide considerable training.

3. Visual scanning and response: The high level of physical fidelity of the KatanaSim enables students to focus on increasing their instrument scanning aptitudes leading to increased performance during slow flight and stall recoveries.

4. Air mass modelling: X-Plane has a detailed weather system which allows an instructor to manipulate all aspects of the weather system from the IOS. Turbulence, wind shear, precipitation, visibility, temperature, and wind speed and direction at low, medium, and high altitudes are just some of the parameters which can be manipulated in order to create highly realistic flight scenarios.

The development of the KatanaSim hardware proved the feasibility of assembling a costeffective, high-fidelity, type-specific simulator which could be used as part of an effective ab-initio flight training program. There were some issues encountered during development, such as unreliable flight and engine instruments from Flight Illusion and SimKits. At various points in time, most of the simulated gauges were prone to some type of failure which 
required frequent re-calibrations, power resets, or consultations with the manufacturers. At present, issues with the gauges have progressed to a point where relatively few of them require additional troubleshooting. Ideally, higher quality instruments would be obtained or designed. The possibility of installing an LCD screen behind the instrument panel in order to display digital instrument faces was discussed as a way of circumventing these issues. If this design change was put into effect, it would be difficult to implement adjustable instrument knobs on the instrument panel such as those located on the altimeter, gyrocompass, and navigation instruments.

Despite these reliability issues, the KatanaSim has the ability to provide very effective training for ab-initio flight students. Future improvements to increase the training effectiveness of the KatanaSim could include a more immersive visual system and the implementation of an active force feedback system for the flight controls. These improvements would be necessary to achieve certification with Transport Canada. 


\section{Chapter 3}

\section{Flight Testing}

The performance of an aircraft can be estimated analytically throughout the design process, but it can only truly be established through flight testing. Flight testing is often performed in the final stages of aircraft development as a way of validating analytical and wind tunnel data and ensuring performance requirements are satisfied. Flight testing is also a critical part of flight simulator development. If there exists published flight performance data for a given aircraft, it is typically only that which is required for piloting the aircraft, such as take-off and landing distances, climb rates, and power requirements. Information regarding flight dynamics, such as velocities and accelerations of the aircraft in flight or pilot inputs required for various manoeuvres, is crucial for flight model and simulator tuning and validation but is typically only available through the aircraft manufacturer at extremely high costs.

Flight test data can be used to validate the accuracy of a flight model, determine overall simulator fidelity, and evaluate training effectiveness. Test data and its role in system identification and flight model validation will be discussed in detail in Chapter 5. 


\subsection{Motivation for Flight Testing}

An accurate flight model is of the utmost importance in achieving a high-fidelity flight simulator, particularly for those like the KatanaSim which are intended for eventual certification. One typical technique for evaluating flight simulators is comparing the handling quality ratings (HQRs) of a simulator with the corresponding aircraft for a given set of manoeuvres. This type of evaluation uses the Cooper-Harper scale to qualitatively assess the vehicle handling characteristics and the demands on a pilot for selected tasks. The Cooper-Harper HQR scale is a subjective rating system used by pilots and flight test engineers to evaluate the ability of the aircraft to achieve certain performance criteria or handle a given manoeuvre, and also accounts for the mental and/or physical demand on a pilot during the tasks.

While this method does give a general impression of the overall simulator fidelity, it does not directly compare the performance of the aircraft with the simulator model. In fact, testing performed at the NASA Ames Research Center proved that changes to the simulator that did not include changes to the flight model, could cause the pilots to assign better or worse handling quality ratings [29]. These changes include reducing the field-ofview and visual resolution, and the motion gains and washout parameters used with the motion cueing system. These results were proven once again in [30] when it was shown that degradations of the visuals in a simulator resulted in pilots assigning lower HQRs to the simulator aircraft. While it is not surprising that changes in the cueing systems of a simulator could cause changes in the perceived HQRs of the aircraft being simulated, it does highlight the need for a method of evaluating the flight model independently of the other simulator systems.

In one simulator fidelity assessment by Beard and Reardon [31], an improvement in simulator fidelity, relative to that achieved by HQR matching, was obtained by matching 
the simulator system response to the response of an aircraft in the same conditions. This method was also implemented successfully in the acceptance testing and commissioning of a rotorcraft flight simulator for the University of Liverpool [4]. In a performance matching analysis, the control inputs of a pilot in the aircraft and in the simulator are compared, along with the response of the aircraft to those inputs. While handling quality ratings can be obtained by interviewing a pilot post-flight, the control inputs and aircraft response required for performance matching must be obtained by instrumenting the aircraft and recording flight control movements and flight dynamics. As performance matching was determined to be the most effective manner for obtaining a high-fidelity flight model but published performance data for the Katana is quite limited, flight testing was deemed necessary.

The development of a general test methodology ensued with the aim of producing a methodology that could be used in small airplane flight testing, particularly for, but not limited to, the Diamond Katana. The test plan was written and the hardware for data collection was designed, assembled, and tested. The data collected from this flight test plan can be used to fully evaluate and validate a flight model.

In addition to flight model validation, the test data has potential to be useful for other research groups in the Mechanical and Aerospace Engineering Department at Carleton University, namely those lead by Professors Craig Merrett and Jeremy Laliberté. As such, these professors and their students also contributed to the development of the test plan and acquisition and installation of equipment.

The KatanaSim is intended for use as part of an ab-initio pilot training program, so it stands to reason that the flight model should accurately model all manoeuvres taught in the course of a PPL training program. As such, the flight test plan was written to include all PPL training program manoeuvres as well as several others for stability analysis purposes. 


\subsection{Test Plan Development}

The flight test plan was developed with help from Adam Vandeven, the Chief Flying Instructor at OAS. The majority of the manoeuvres were chosen based on the student pilot training curriculum at OAS and the remaining manoeuvres, such as the flight stability and phugoid* tests, were chosen to test the stability and control characteristics of the aircraft. The During the flight testing, data would be recorded as per standard flight testing methods described in [32]. Due to the number of manoeuvres and the time it would take to complete all of them, it was decided there would be three test flights, with the manoeuvres ordered as shown in Table 3.1.

Table 3.1: Flight test manoeuvres including flight time [17].

\begin{tabular}{|c|c||c|c||c|c|}
\hline \multicolumn{2}{|c||}{ Flight 1 } & \multicolumn{2}{c||}{ Flight 2 } & \multicolumn{2}{c|}{ Flight 3 } \\
\hline $\begin{array}{c}\text { Time } \\
(\mathrm{mins})\end{array}$ & Task & $\begin{array}{c}\text { Time } \\
(\mathrm{mins})\end{array}$ & Task & $\begin{array}{c}\text { Time } \\
(\mathrm{mins})\end{array}$ & Task \\
\hline \hline 10 & Start \& T/O & 10 & Start \& T/O & 10 & Start \& T/O \\
\hline 10 & Transit & 10 & Transit & 10 & Transit \\
\hline 5 & High, level & 5 & Low, level & 3 & Flight stability \\
\hline 12 & Phugoid & 10 & Climb & 12 & Phugoid \\
\hline 3 & Flight stability & 3 & Flight stability & 6 & Short period \\
\hline 6 & Short period & 12 & Phugoid & 5 & Slips \\
\hline 10 & Stall & 6 & Short period & 25 & Circuits \\
\hline 5 & Spin & 5 & Turns & 10 & Transit \\
\hline 5 & Slow flight & 5 & Slips & 5 & Landing \\
\hline 7 & Spiral dive & 10 & Transit & & \\
\hline 5 & Slips & 5 & Landing & & \\
\hline 10 & Transit & & & & \\
\hline 5 & Landing & & & & \\
\hline \hline 93 & Total time & 81 & Total time & 86 & Total time \\
\hline
\end{tabular}

*A phugoid is a long period oscillation in which the aircraft follows a sinusoidal flight path. 


\subsection{Equipment and Installation}

Data would be gathered throughout the test flights using an assortment of equipment installed in the aircraft as well as a clipboard to manually record prevailing operating conditions and parameters.

A detailed explanation of the test plan and the entire equipment setup can be found in [17]. The following sections detail certain aspects of the equipment as well as the process by which the equipment was tested and certified. Work that was completed by other research assistants is not detailed here.

The parameters of interest and the requisite equipment are listed in Table 3.2.

Table 3.2: Equipment required for recording flight test data.

\begin{tabular}{ll}
\hline Equipment & Function \\
\hline XSens MTi Motion Tracker (IMU) & Linear and angular accelerations \\
ArduPilot Mega (redundant IMU) & Linear and angular accelerations \\
Freescale MPXV7002 air pressure sensor & Airspeed \\
Mediatek GPS & Location and altitude \\
Intertechnology SP3-12 string potentiometers & Control inputs \\
Custom circuit board & Required for string potentiometers \\
Arduino UNO microcontroller board & Data gathering (string potentiometers) \\
GoPro "Hero 2" & Audio-visual record of flight \\
Laptop & Data recording \\
Data marker button & Create data markers during flight \\
Batteries & Power for components \\
Clipboard, sortie cards & Manual data recording \\
\hline
\end{tabular}

\subsubsection{Equipment Mount}

A $0.48 \mathrm{~m} \mathrm{x} 0.61 \mathrm{~m}$ (19 inch x $24 \mathrm{inch})$ instrumentation interface board made of plywood was installed in the baggage compartment of the Katana. The board was mounted on four 5 inch lag bolts to increase height and facilitate securing; and the corners of the 


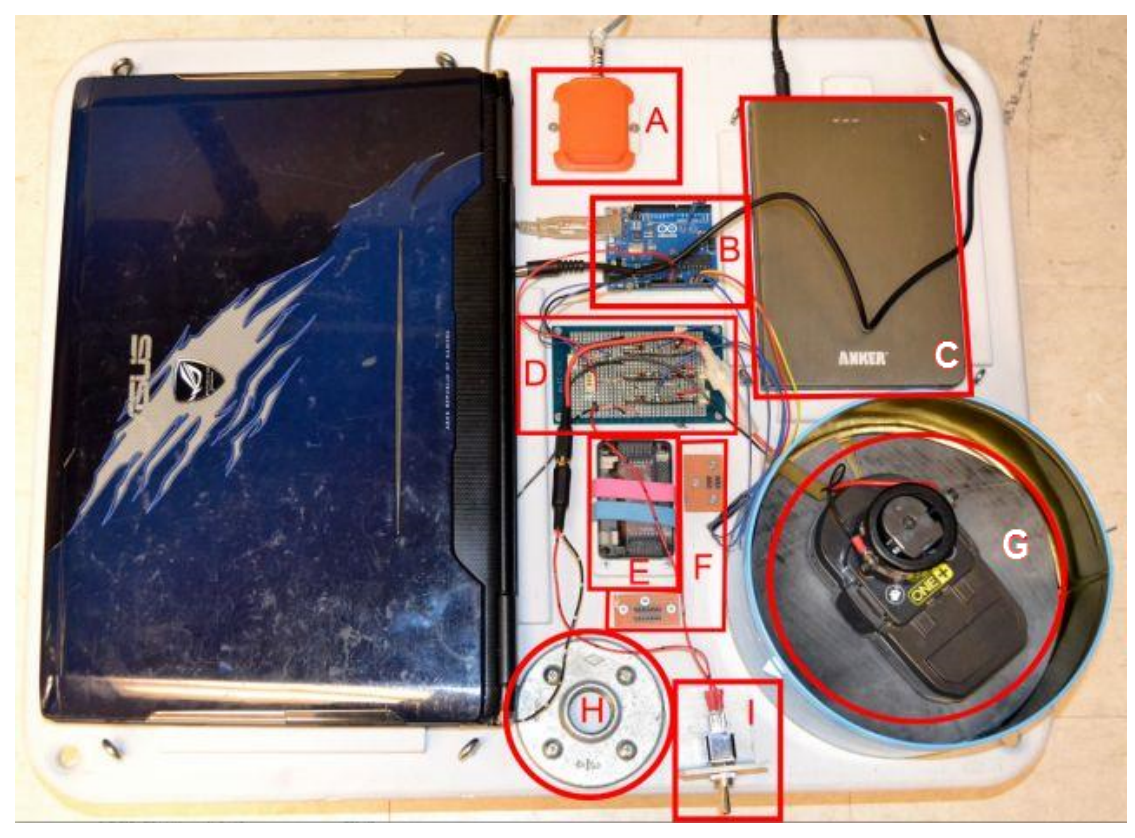

Figure 3.1: Equipment mount to be installed in the baggage compartment during flight test with laptop and other equipment. Labels: A - XSens MTi sensor, B - Arduino UNO C - External laptop battery, D - Custom circuit board, E - ArduPilot Mega, F - Connectors for GPS and air pressure sensor wiring, G - Battery in rubber lined metal enclosure, $\mathrm{H}$ Base of GoPro mount, I - Power switch for string potentiometers.

board were securely tied down using parachute cord attached to brackets installed in the baggage compartment. Zip ties were used as a redundant securing system. This board acted as the mounting point for the XSens IMU, custom-built circuit boards, batteries, and recording equipment required for the flight test. The layout of the equipment can be seen in Figure 3.1 and the installation of the board in the Katana can be seen in Figure 3.2.

\subsubsection{Data Recording Devices}

Throughout the flight, data were recorded on a laptop which was tied securely to the equipment mount installed in the baggage compartment of the Katana. All recording software was acquired, modified, and tested by Jonathan Plumpton [22]. Additionally, a video and audio recording of the cockpit during flight test was made using a GoPro Hero 2 camera with a fish eye lens. The camera was mounted on a rigid support cantilevered 

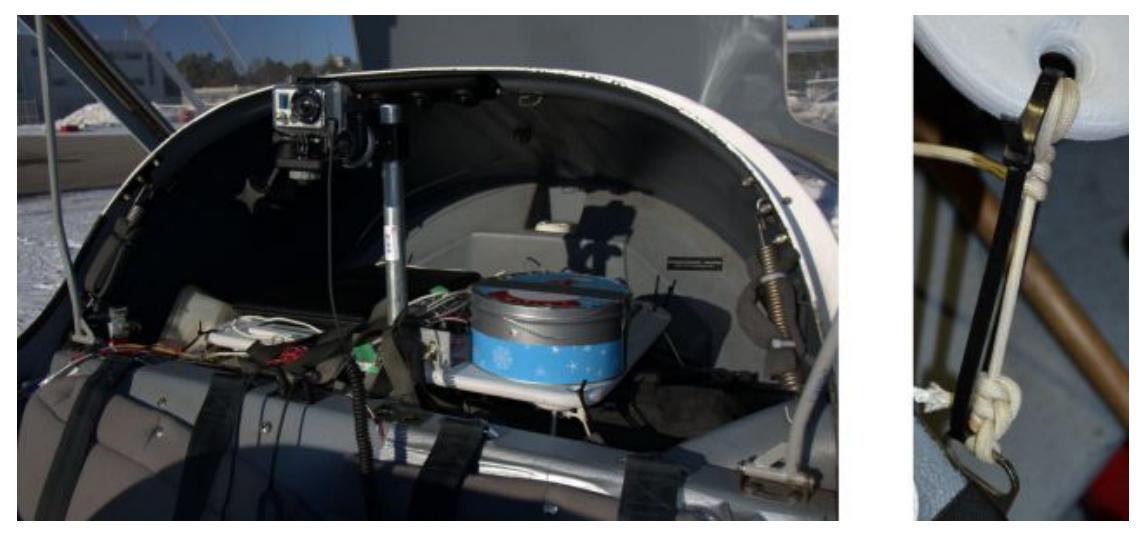

Figure 3.2: Left: Equipment mount secured in baggage compartment just prior to take-off. Right: One corner of the equipment mount, secured with both a zip tie and parachute cord tied with a turn and three half hitches.

to the equipment board in the cargo compartment, from which the camera line of sight was over the shoulders of the crew members to the instrument panel. As the ambient noise in the cockpit during flight is very loud, an audio cable was used to connect the GoPro directly to the headset and radio system allowing a recording to be made of all conversations and radio transmissions throughout the flight.

As the laptop was closed and installed in the baggage compartment, there was no way of using it directly to confirm that the system was still running throughout the flight or to add data markers periodically to the recorded data throughout the flight. To remedy the first of these issues, a small LED was connected to the system which would flash periodically as long as the system was functioning. This LED was installed in a tethered film canister along with a small push button that could be used to create data markers to mark the beginning and end of each manoeuvre in the data file. This film canister was connected to the board by long wires running forward in the cockpit so that the button and LED were easily accessible to the flight crew member in the right seat. During the flight test, the cannister was secured to the right leg of the crew member. 


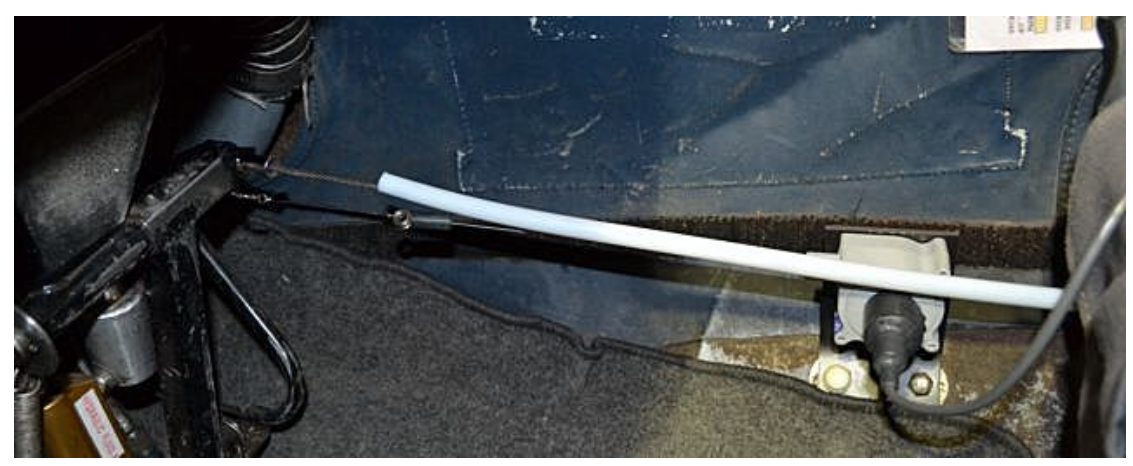

Figure 3.3: String potentiometer attached to right co-pilot rudder pedal to record rudder movements during flight test.

\subsubsection{Control Inputs}

In order to record the position of the control columns and rudder pedals at all times during the flight test, string potentiometers were installed in the cockpit. The certification aspects of this set-up are discussed in Section 3.4.

One string potentiometer was installed near the floor on the right side cockpit wall. The string from this potentiometer extended nearly parallel to the rudder cable and was zip tied to the pedal in order to directly measure rudder input. This set up can be seen in Figure 3.3.

The control column, which has two degrees of freedom, required two string potentiometers to accurately capture the roll and pitch inputs of the pilot. The two potentiometers were clamped to the underside of the instrument panel using custom built clamps, similar to C-clamps, as pictured in Figure 3.4.

The strings from the potentiometers extended aft to the control column where they were zip tied tightly just beneath the handle of the column, as shown in Figure 3.5. These two potentiometers were installed carefully to minimize interference with the legs of the crew member in the right seat of the cockpit.

The string potentiometers required a minimum of $12 \mathrm{~V}$ to operate, which could not be provided by the ArduPilot microcontroller. A separate system was designed in which the 


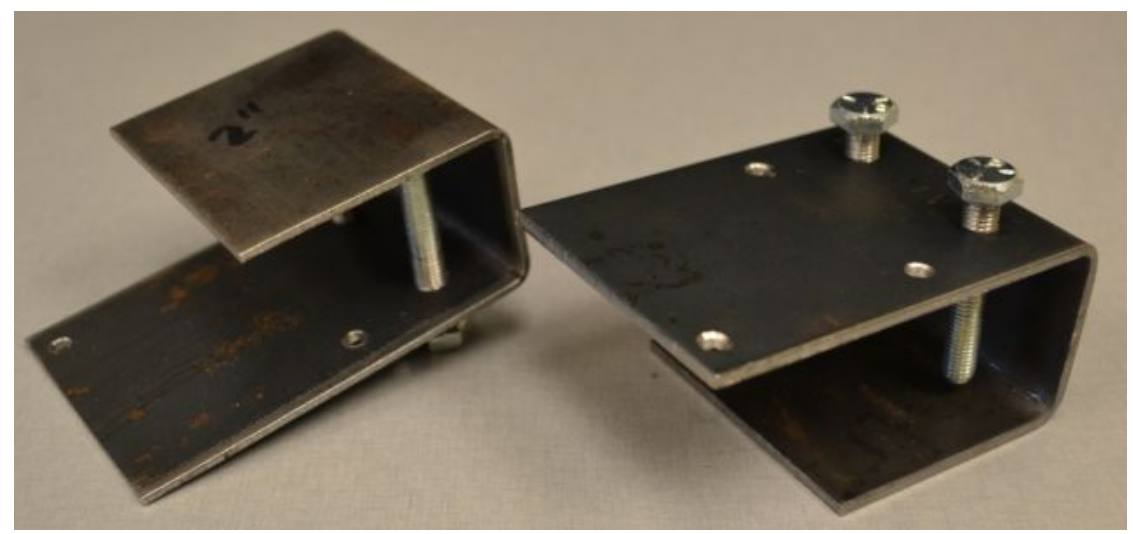

Figure 3.4: Custom-built clamps used for string potentiometer installation on underside of instrument panel.

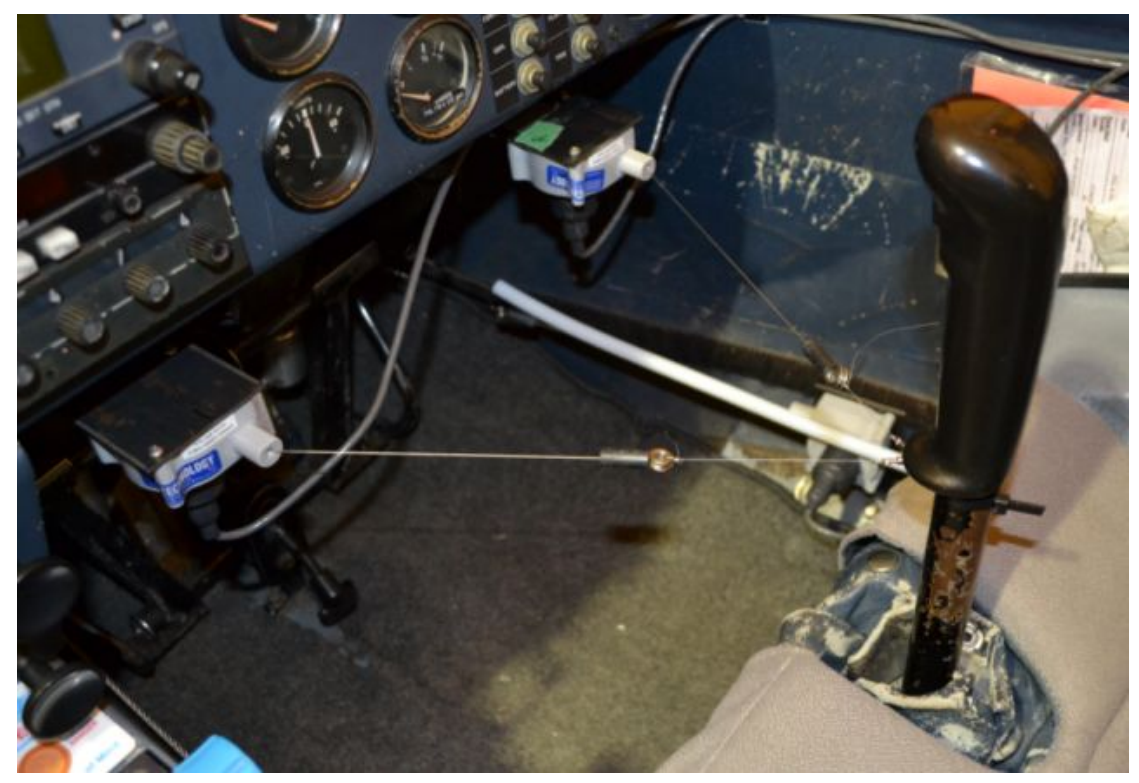

Figure 3.5: String potentiometers installed under the instrument panel in front of the co-pilot to record control column movements during flight test. 
potentiometers drew power from an external battery. A custom circuit board (Figure 3.6) was created that provided ground and power connections for the string potentiometers. The board also hosted three voltage dividers, one for each string potentiometer, which were required in order for the potentiometer data to be readable by the Arduino UNO microcontroller board to which it was connected.

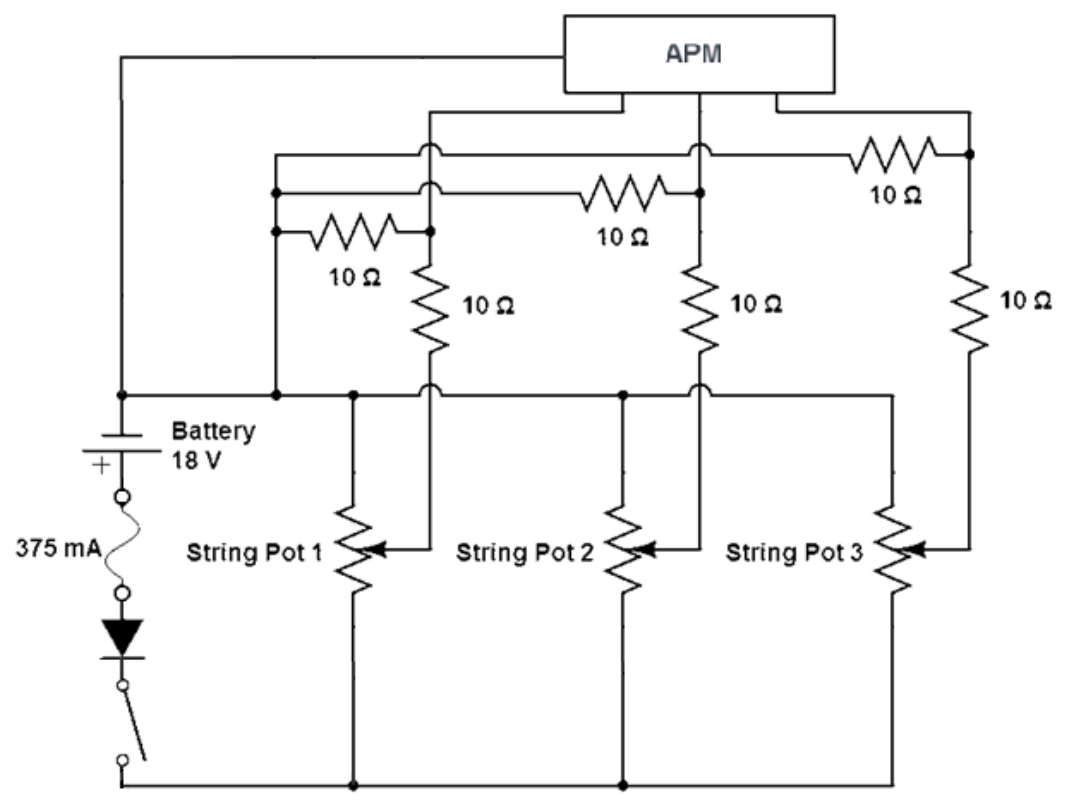

Figure 3.6: Circuit diagram for the three string potentiometers, the power source, and the voltage dividers.

The data from the string potentiometers were fed through the circuit board to the ArduPilot Mega (APM) and then recorded on the laptop at $60 \mathrm{~Hz}$. The data files were translated to roll, pitch, and yaw deflection fractions in post-processing using the method described in Section 3.7.1.

\subsubsection{Sensors}

Before the flight test, the airspeed sensor was installed in the aircraft and integrated into the pitot-static system by means of tees installed in the pressure lines. This installation was done by a certified aircraft maintenance engineer (AME) and, as is required after any 
work on the pitot-static system is completed, the entire system was tested to ensure no air leaks were present in the system. The airspeed sensor, along with the the Mediatek GPS which was secured to the top of the instrument panel, interfaced with the APM to record location and airspeed data. Aircraft accelerations and orientations were recorded by both the APM and the XSens MTi Motion Tracker. An in-depth description of these four components (the APM, airspeed sensor, Mediatek GPS, and XSens MTi Motion Tracker) can be found in [17].

\subsection{Safety and Certification}

Flight testing can be a dangerous endeavor due to edge-of-envelope manoeuvres, the equipment modifications necessary to install test equipment, and the experimental nature of the aircraft being flown. Flight testing in Canada is closely regulated by Transport Canada to ensure safety is paramount in all instances of flight testing.

As the flight testing in question was to be performed on an in-service certified aircraft, the risks of flying an experimental aircraft were eliminated. Risks were further reduced by ensuring all manoeuvres performed were within the regular performance envelope of the aircraft. Proper checks were written into the flight plan, extra emergency procedures made known, and Adam Vandeven, the Chief Flying Instructor at OAS, was heavily consulted during the design of the three flight plans. The remaining risks which were of most concern to Transport Canada and all members of the flight test team were those pertaining to the equipment being installed in the aircraft, most notably the batteries and the string potentiometer connections with the flight controls. 


\subsubsection{Equipment Design}

\section{String Potentiometer Safety Features}

The string potentiometers which were used to record the movements of the rudder pedals and control column interfaced directly with the flight controls. Precautions were taken to ensure the string potentiometers did not interfere with the ability of the pilot to control the aircraft during regular flight, as well as to minimize the risk that a jammed potentiometer would affect the mobility of the flight controls.

The two string potentiometers used to measure the motion of the control column were installed on the co-pilot's side of the aircraft in order to eliminate any interference with the pilot's movements, and in such an area as to minimize any risk of interaction between the strings and the legs of the co-pilot, though this type of interaction would not affect the control of the aircraft in any way. The two strings were secured to the base of the right-side control column handle with a zip tie. Short lengths of fishing line were included between the ends of each of the strings and the zip tie to act as weak links in the connection. The weak links ensured that if the string potentiometers were to jam, the pilot could give the control column a quick tug and the strings would break with less than $89.3 \mathrm{~N}$ (20 lbs) of applied force.

The third string potentiometer, used to record rudder inputs, was installed between the rudder cable and the interior fuselage wall near the right foot of the crew member in the right seat of the cockpit. This location effectively eliminated any chance of the string potentiometer interfering with the pilot's or co-pilot's movements. As with the other two string potentiometers, a small length of fishing line was used as a weak link between the potentiometer string and the corner of the right rudder pedal to which it was zip tied. 


\section{Batteries and Emergency Procedures}

The longest of the flights (including run-up and taxi time) was estimated to be 93 minutes, and with a buffer to account for equipment setup, calibration times, and any possible small delays, it was decided that the batteries needed to provide enough power for a minimum of 120 minutes. Transport Canada regulates the use of certain types of batteries under the Transportation of Dangerous Goods Act and Regulations and enforces a requirement that Li-ion batteries have ratings of no more than $100 \mathrm{Wh}$ and $7800 \mathrm{mAh}$ [33]. These considerations were taken into account during battery selection and testing.

Three batteries were required to provide sufficient power to the setup for the required time. The first battery was the Li-ion laptop battery, which met all regulations for use in an aircraft cabin during flight and would be protected from short-circuiting by its location inside the laptop. For the laptop, an additional power source was required to meet the time requirements. An Anker Astro Pro2 multi-voltage external battery was acquired to provide the additional power. The casing of the external battery was deemed sufficiently safe for use in the aircraft during flight and a telephone call to Anker confirmed that the $3800 \mathrm{mAh}$ rating for the external battery was well below the Transport Canada limit of $7800 \mathrm{mAh}$.

The third battery was used as a power source for the string potentiometers, LED status light, and data marker push button. As the string potentiometers required a minimum of $12 \mathrm{~V}$, an $18 \mathrm{~V} \mathrm{Li}$-ion battery was found to be an acceptable power source. The $24 \mathrm{Wh}$ capacity and $1333 \mathrm{mAh}$ rating were both well within acceptable ranges outlined by TC. This battery was found to be acceptable for use in the aircraft during flight, but certain safety measures first had to be implemented to mitigate the risk of a battery fire as the casing alone was deemed insufficient protection in case of fire. The battery was installed in a rubber-lined sheet metal enclosure and an emergency procedure, found in Appendix B was developed and documented for use in the case of a battery fire during flight. 


\subsubsection{Flight Permit Acquisition}

The majority of the flight testing equipment systems were self-contained and would not have required a TC Flight Permit had it not been for the air pressure sensor and the string potentiometers. The definition of a major modification, according to Transport Canada, is an "alteration to Type Design of an aeronautical product in respect of which a Type Certificate was issued that has other than negligible effect on weight, centre of gravity limits, structural strength, performance, powerplant operation, flight characteristics, or other qualities affecting its airworthiness or environmental characteristics" [33]. While the string potentiometers were not believed to affect any of these characteristics, it was clear that interfacing them directly with the flight controls would likely require TC approval. The airspeed sensor was of even greater concern as it was integrated into the pitot-static system of the aircraft. As the integrity of the pitot-static system is absolutely imperative for flight safety, as are unimpeded flight controls, a flight permit application was filed.

Assistance from Ashraf Othman, a Transport Canada Design Approval Representative and certification consultant, was key in obtaining approval for the flight permit application. He provided significant guidance in the creation of a test plan report and handled the direct communications with representatives at Transport Canada. Chris Adams, the OAS Director of Maintenance, also helped in liaising with Transport Canada to obtain the final flight permit approval.

Transport Canada representatives raised the various batteries as a potential issue, but the issue was satisfactorily resolved when safety precautions, such as an insulated battery enclosure, had been implemented and the emergency procedure was written for an in-cabin battery fire. The flight permit was successfully acquired and can be found in Appendix C. 


\subsection{Equipment Testing}

Pre-flight testing of the equipment was carried out in several stages. Initially, all of the equipment and sensors were tested separately to ensure functionality. This included testing the airspeed sensor with a manometer to ensure correct readings could be obtained up to its maximum rating of 120 knots. The airspeed of the Katana was not expected to exceed 120 knots during flight as 118 knots is the maximum structural cruising speed allowed; any faster airspeeds introduce a risk of structural damage. Once the individual equipment functions had been confirmed, all of the equipment was connected at once to the laptop to ensure the software could handle the inputs coming from all of the sensors and that data was being recorded at a frequency of $60 \mathrm{~Hz}$.

The second stage of testing was performed outside of the laboratory so that additional testing could be performed on the airspeed sensor and GPS, as well as the battery life. The full equipment board was installed in the back seat of a car with the GPS and GoPro secured to the dashboard of the car, and a pitot tube was secured to the roof on the car exterior. The pitot tube lines ran through a narrow opening in the car window and were connected to the airspeed sensor. The string potentiometers were also present and connected to the system, though the strings were not attached to anything. The car was then driven at a variety of speeds. It was confirmed that the airspeed, GPS, and indicated ground speed of the vehicle were in agreement. The strings on the string potentiometer were also periodically stretched to various lengths to ensure proper function. This test took place over the course of two hours with no battery life issues observed.

A third stage of testing was performed to investigate the effect of cold temperatures on the system. Katanas are limited in cold weather due to their composite structures and are not considered airworthy in temperatures of $-20^{\circ} \mathrm{C}$ or less. Even so, it was decided that the equipment should be tested in the cold to see how the various batteries were affected 
and what charging times would be sufficient. As the charging would be indoors, there was also some concern about whether the temperature changes would negatively affect the equipment. A January day was chosen with a temperature of $-18^{\circ} \mathrm{C}$. The equipment was placed outside in a stationary box and successfully ran for two and a half hours. The laptop and external batteries were then charged for 65 minutes, an attempt to emulate an abbreviated charging period between flights. After this, the equipment was hooked up once again. The laptop ran for 100 minutes on the external battery before switching to the laptop battery. Based on this, the minimum battery charging time between flights was set at 75 minutes.

The GoPro was also tested for cold weather performance. In the same $-18^{\circ} \mathrm{C}$ weather and with a fully charged battery, the GoPro was able to record 135 minutes of video. As this exceeded the 120 minute minimum, this was considered acceptable. However, after the battery was charged for 65 minutes, the GoPro shut down after only 87 minutes of recording. During that time, two video files were created with time stamps indicating that the GoPro had stopped recording for a 75 minute period in between the other two recordings. As this was not acceptable, a new extended life battery was obtained for the GoPro which provided ample battery life for the flight tests.

\subsection{Flight Test}

The day before flight testing was scheduled to occur, the equipment was tested for proper fit in the Katana cockpit and the airspeed sensor was given to the AME so that installation of the sensor and a pitot-static system test could be performed in the evening. As the aircraft was scheduled for a possible flight later that day, no equipment could actually be installed.

On the morning of flight testing, March 8th 2014, equipment installation began at 


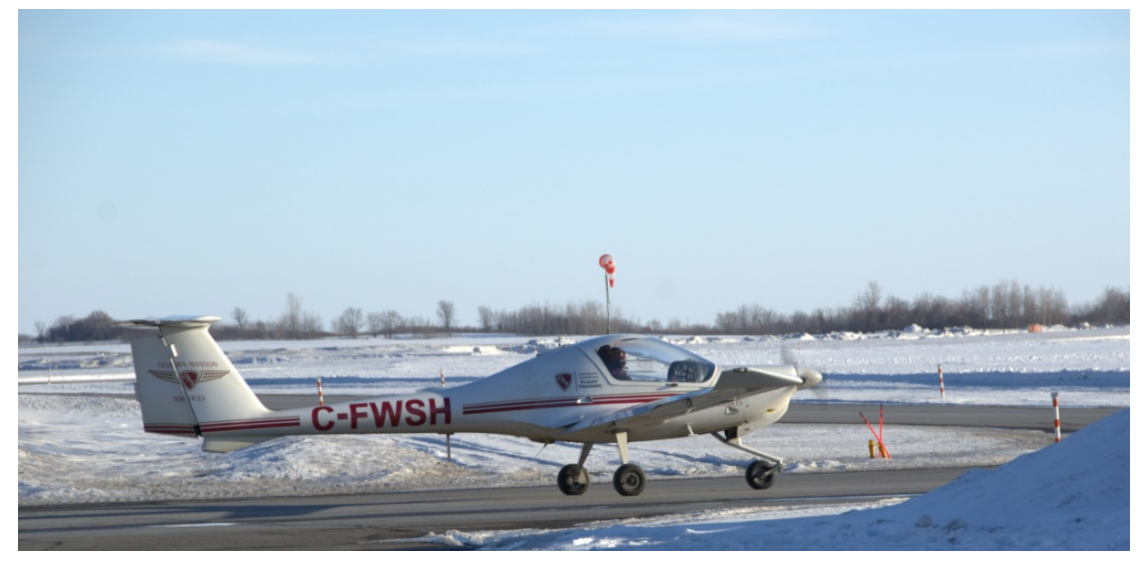

Figure 3.7: The fully-equipped Katana taxiing out for the first flight test.

7 AM. Due to both installation time and poor morning weather, the first flight took off at noon (Figure 3.7). By this time, the cloud ceiling had lifted, precipitation had stopped, and the weather had become nearly perfect; there was no ceiling at all, few clouds, and only mild turbulence. The first flight was 2.5 hours long and was performed precisely as described in the flight plan. No issues were encountered during the flight.

After the first flight landed, all of the batteries were charged and the data were downloaded. A brief check showed that all of the sensors had been recording data and the GoPro had a clear audio and visual recording (Figure 3.8). The only error found at this point was the lack of data markers; the connectors for the data marker had come loose. This connection was repaired with electrical tape for the second flight.

As the first flight had been flown at a later time of day than anticipated, concerns were expressed about the ability to complete two more test flights as the battery charging time between flights would push the third flight well into the evening after dark. Both the aircraft and the pilot were rated to fly in the dark, and the flight test permit did not specify day or night limitations, but everyone involved agreed it would not be wise to be performing the test manoeuvres at night. The third flight had been intended to gather additional data on manoeuvres that were all scheduled to be performed in the first and second flights, with the exception of 5 circuits which had been added to provide ample 


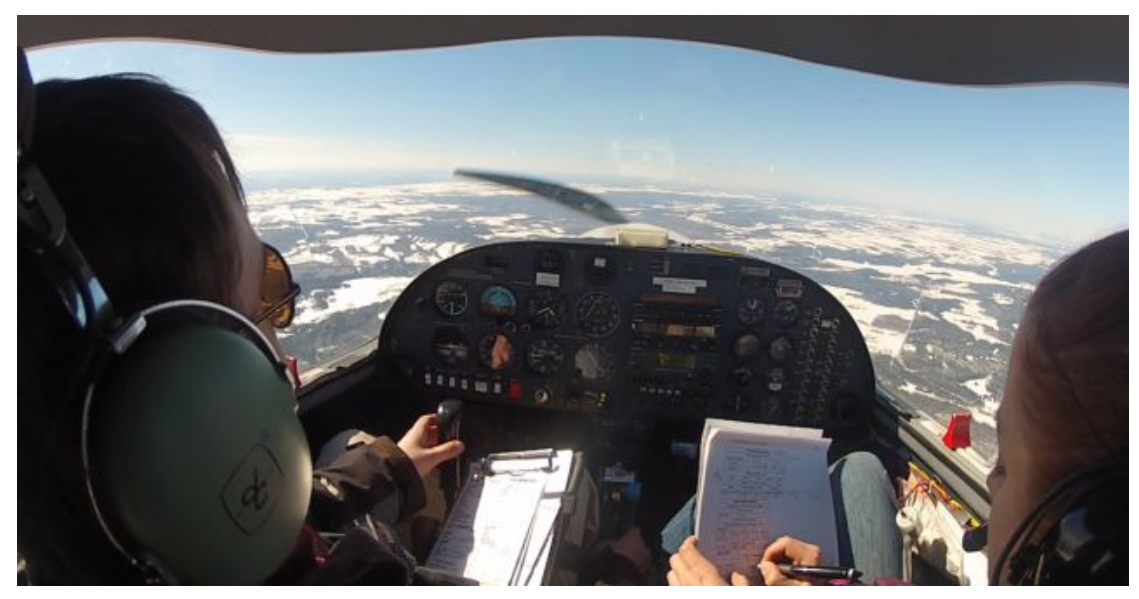

Figure 3.8: Image from the GoPro video recording of the flight test.

take-off and landing data sets. It was concluded that the charging time of the batteries would be extended by an hour, and the second flight would be flown with the additional circuits added to the list of manoeuvres. As a result, it was possible to remove the third flight from the test plan.

The second flight also went very well. The weather continued to be favourable and even less turbulence was experienced than during the first flight. One modification was made to the flight plan after take-off. A change in the order of manoeuvres was made to more easily accommodate altitude requirements. The only small issue during the flight was a 5 second period of interference between the crew member's leg and the left string potentiometer. As this interference was during a period in which the control column was being held steady, the corrupted data was easily repaired during post-processing. The raw data sheets from the two flights can be found in Appendix D.

\subsection{Data Processing and Analysis}

In the days following the flight test, the flight test data were carefully examined and post-processing began for certain data sets. 


\subsubsection{Control Column and Pedal Inputs}

The data from the string potentiometers attached to the flight controls were recorded at $60 \mathrm{~Hz}$. Each data point consisted of a number between 0 and 1024, indicative of the outstretched length of the string at that instant. The positions of the controls were defined as fractions of deflection with the neutral positions of the stick and pedals chosen as zero. Full right rudder, full right roll, and full pitch down were all defined as 1.0 while full left rudder, full left roll, and full pitch up were all defined as -1.0. These coordinates were chosen in order to match X-Plane's system of defining control column and rudder pedal position.

The position of the rudder was easy to obtain from the string potentiometer data. Initial readings were taken to establish the maximum string length $L_{\max }$ corresponding to full right rudder, and minimum string length $L_{\min }$ corresponding to full left rudder. The length of the string at any moment $L$ in time could then be used to calculate the deflection fraction $\Delta$ of the rudder, using

$$
\Delta=\frac{L-L_{\min }}{L_{\max }-L_{\min }} * 2-1
$$

where 1.0 and -1.0 indicated full right and full left rudder respectively.

The position of the control column required a more complex set of equations. A diagram of the control column structure is shown in Figure 3.9. The control column has a handle at the top for the pilot, and a pin joint (Point $B$ ) on a frame near the base of the column about which the stick can pivot left and right as the pilot rolls the aircraft. The small frame which supports this pin joint is in turn connected to an inner aircraft wall by another pin joint (Point $A$ ). This pin allows the frame and the control column it contains to pivot fore and aft as the pilot controls the pitch of the aircraft. Point $C$ was located directly beneath the control column grip and was the point where the two strings from the 
potentiometers were attached. Point $D$ was defined as the point on the right-most string potentiometer where the string left the housing.

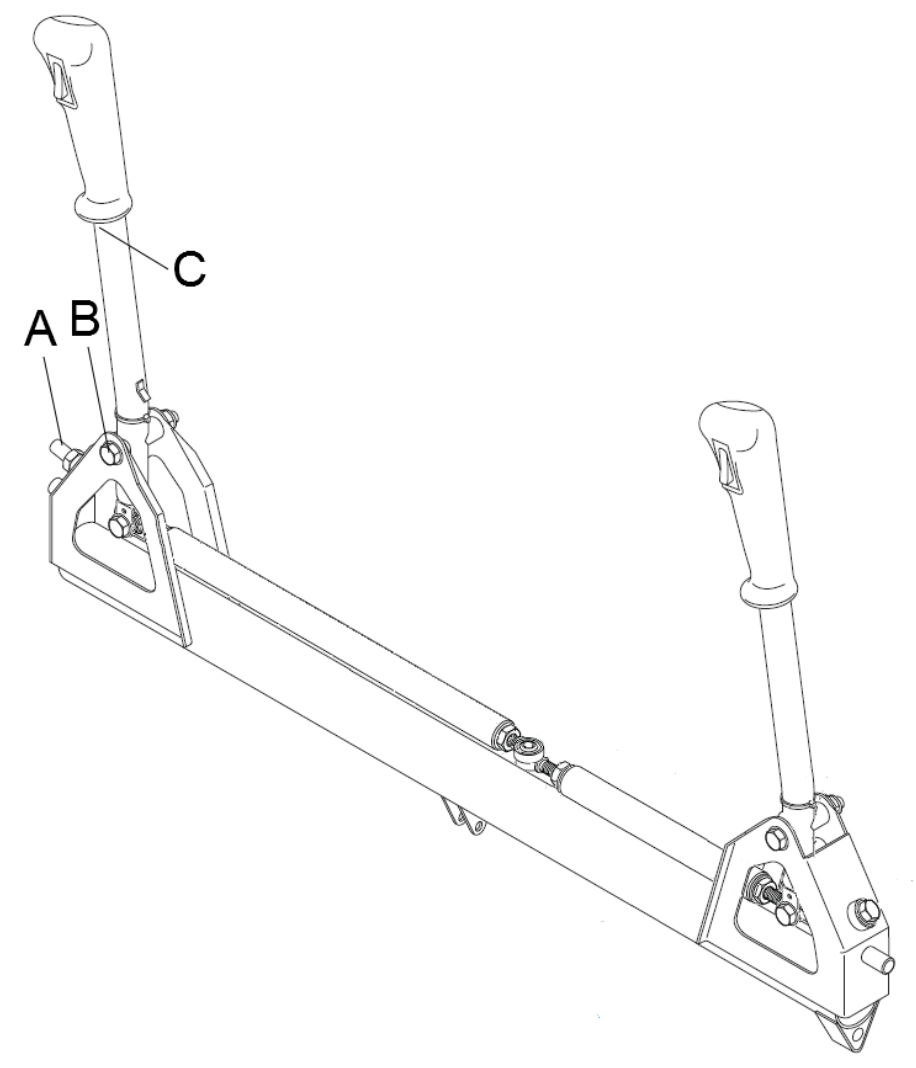

Figure 3.9: Control column design, modified from the Diamond Katana DA20-A1 Illustrated Parts Catalogue [34]. Point A - Pin joint about which the control column rotates during pitch inputs, Point B - Pin joint about which the control column rotates during roll inputs, Point $\mathrm{C}$ - Connection point of the strings from the potentiometers to the control column.

It should be noted that the control column, when in the neutral position, is not vertical; the upper portion of the control column angles slightly forward. The method used for modelling the control column is shown in Figure 3.10. The control column was modelled as two links, joined by pin joints. Link 1 (L1) extended from Point A to Point B, and Link 2 (L2) extended from Point B to Point C.

In order to write the required kinematic equations, the system was defined using three right-handed coordinate systems. The first of these, AC, was fixed to the aircraft. The 


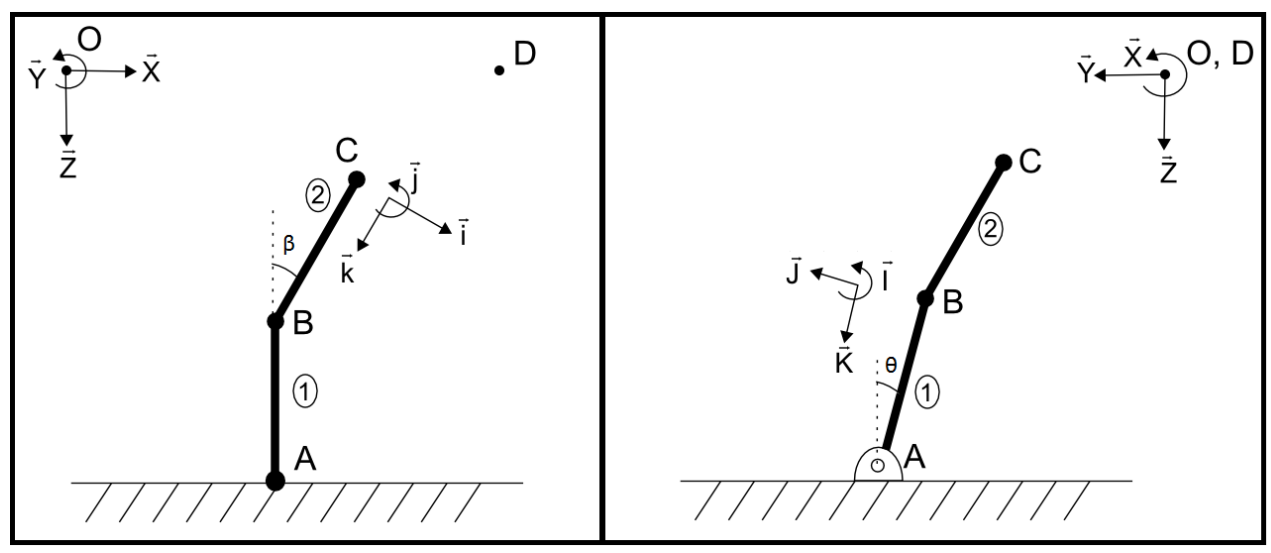

Figure 3.10: Front and side view of control column model used for post-processing of data to acquire control column position during flight test.

origin of the system, Point $O$, was at the left string potentiometer, at the point where the string exited the potentiometer housing. The system is defined by the $\vec{X} \cdot \vec{Y} \cdot \vec{Z}$ unit vector triad where $\vec{X}$ and $\vec{Y}$ lie in a horizontal plane, $\vec{X}$ is positive to the right, and $\vec{Z}$ is positive down.

Point $A$ was located at the bottom pivot of the control column model, and served as the origin for the L1 coordinate system, defined by $\vec{I} \cdot \vec{J} \cdot \vec{K}$. The positive $\vec{K}$ axis ran along Link 1 from Point $B$ to Point $A$. The positive $\vec{I}$ axis ran parallel to the $\vec{X}$ axis in the AC system.

The third coordinate system, L2, was fixed at Point $B$ and was defined by the $\vec{i} \cdot \vec{j} \cdot \vec{k}$ triad. Unit vector $\vec{k}$ was positive along Link 2 from Point $\mathrm{C}$ to Point B and $\vec{j}$ ran parallel to the $\vec{Y}$ axis in the AC system.

Angle $\theta$ was defined as the angle of rotation of L1 from vertical due to pitch input, around the $\vec{X}$ axis of the AC coordinate system. Angle $\beta$ was defined as the angle of rotation of L2 from the neutral position due to roll input around the $\vec{j}$ axis of the L2 system.

In tracking the position of the control column, the first step was to describe the position vector of Point $C$ with respect to Point $O$, 


$$
\vec{R}_{C / O}^{A C}=\vec{R}_{B / A}^{L_{1}}+\vec{R}_{C / B}^{L_{2}}+\vec{R}_{A / O}^{A C}
$$

where the superscript of position vector "R" denotes the coordinate system in which it is defined.

Rotational transformation matrices were then added to change all position vectors to the AC coordinate system

$$
\vec{R}_{C / O}^{A C}=\left[T_{L 1 A C}\right] \vec{R}_{B / A}^{L_{1}}+\left[T_{L 1 A C}\right]\left[T_{L 2 L 1}\right] \vec{R}_{C / B}^{L_{2}}+\vec{R}_{A / O}^{A C}
$$

where the subscripts of the transformation matrices describe the resulting transformation, i.e. $\left[T_{L 1 A C}\right]$ denotes a transformation from the $\mathrm{L} 1$ coordinate system to the $\mathrm{AC}$ coordinate system. This equation was then expanded, resulting in

$$
\vec{R}_{C / O}^{A C}=\left[\begin{array}{c}
L_{A O_{x}}+L_{B C_{k}} \sin \beta \\
L_{A O_{y}}-L_{A B_{K}} \sin \theta+L_{B C_{j}} \cos \theta-L_{B C_{k}} \cos \beta \sin \theta \\
L_{A O_{z}}+L_{A B_{K}} \cos \theta+L_{B C_{j}} \sin \theta-L_{B C_{k}} \cos \beta \cos \theta
\end{array}\right]=\left[\begin{array}{c}
x_{i n t} \\
y_{i n t} \\
z_{i n t}
\end{array}\right]
$$

where $x_{i n t}, y_{\text {int }}$, and $z_{\text {int }}$ represent the coordinates of Point $\mathrm{C}$, and the $\mathrm{x}, \mathrm{y}, \mathrm{z}$ distances between points $\mathrm{O}, \mathrm{A}, \mathrm{B}$, and $\mathrm{C}$ are denoted by $\mathrm{L}$ variables and their appropriate subscripts.

A second equation for $x_{i n t}$ was written using the equation for the intersection plane of two spheres. These spheres represent the possible locations of the ends of the strings from the potentiometers given the lengths of the strings. As the ends of the two strings were tied together, their intersection point had to lie somewhere on a given y-z plane, located at $x=x_{i n t}$. The resulting equation for $x_{i n t}$ is

$$
x_{i n t}=\frac{R_{D / O}^{2}+r_{O}^{2}-r_{D / O}^{2}}{2 R_{D}}
$$


where $R_{D / O}$ is the distance between the string potentiometers, $r_{D}$ is the radius of the sphere of possible points given by the length of the string potentiometer 2 located at Point $D$, and $r_{O}$ is the radius of the sphere of possible points given by the length of string potentiometer 1 (located at the origin). The string potentiometer data gave $r_{D}$ and $r_{O} ; R_{D / O}$ was known from measurements taken during installation of the string potentiometers.

Knowing $x_{\text {int }}$, angle $\beta$ was then found using the first row of Equation 3.4,

$$
\beta=\arcsin \left(\frac{x_{i n t}-L_{A O_{x}}}{L_{B C_{k}}}\right)
$$

The only remaining unknown variable of interest, angle $\theta$, could then be solved. An equation was written corresponding all possible locations for Point $C$, based on a sphere with centre at point $\mathrm{O}$,

$$
r_{O}^{2}=x_{i n t}^{2}+y_{i n t}^{2}+z_{i n t}^{2}
$$

which was rearranged to create the function

$$
f(\theta)=r_{O}^{2}-x_{i n t}^{2}-y_{i n t}^{2}-z_{i n t}^{2}
$$

This equation was expanded using the definitions of $\beta, x_{i n t}, y_{i n t}$, and $z_{i n t}$ from Equations 3.4, 3.5, and 3.6. The function $f(\theta)$ was then differentiated to find $f^{\prime}(\theta)$. The lengthy final forms of the functions $f(\theta)$ and $f^{\prime}(\theta)$ can be found in Appendix E.

Once $f(\theta)$ and $f^{\prime}(\theta)$ were known, the Newton-Raphson method was used to determine the root of the equation which corresponded to the $\theta$ angle of the control column. The Newton-Raphson method is an iterative process which finds progressively closer approximations to the roots of a given function, in this case $f(\theta)$. A first guess for the root, $\theta_{0}$, was selected. An approximation of the root is then calculated as: 


$$
\theta_{n+1}=\theta_{n}-\frac{f\left(\theta_{n}\right)}{f^{\prime}\left(\theta_{n}\right)}
$$

This process was repeated until the root of the function was found. As there are two roots to this equation, the initial $\theta_{0}$ was chosen to be the converged $\theta$ value from the previous set of data points to avoid convergence to the incorrect root on subsequent solutions.

In the initial phase of this program, angles $\theta$ and $\beta$ were used to calculate the $\mathrm{X}$ and $\mathrm{Y}$ coordinates of Point $\mathrm{C}$. The $\mathrm{X}$ and $\mathrm{Y}$ coordinates were then compared to the maximum and minimum $\mathrm{X}$ and $\mathrm{Y}$ values in order to determine the pitch and roll deflection fractions. It was decided after validation testing to revert to using angles $\theta$ and $\beta$ directly to find the deflection fractions. This was decided based on the ease of calculating the deflection fraction of the control surfaces based on the input angles; the previous calculation method did not properly take into account the slight changes in vertical position $Z$.

\section{Validation}

Calibration trials were run in advance of the flight test to ensure the above method produced accurate results. A trial was performed in which the perimeter of the range of the control column was mapped, in effect tracing a rectangle. After the perimeter was traced, the control column was moved in a spiral and returned to an approximately neutral position. The data points recorded were filtered using a 10-point moving average filter to remove the noise in the data, with each filtered point being an average of the five previous and five following points. The data points from the validation test were plotted and can be seen in Figure 3.11 where the rectangle represents the actual perimeter of the range of the control column.

The calculated data points had a definite skew, with discrepancies as much as a $1.25 \mathrm{~cm}$ between the calculated data point and actual physical location. It was determined that the discrepancies were the result of inaccurate measurements of the system geometry. The 


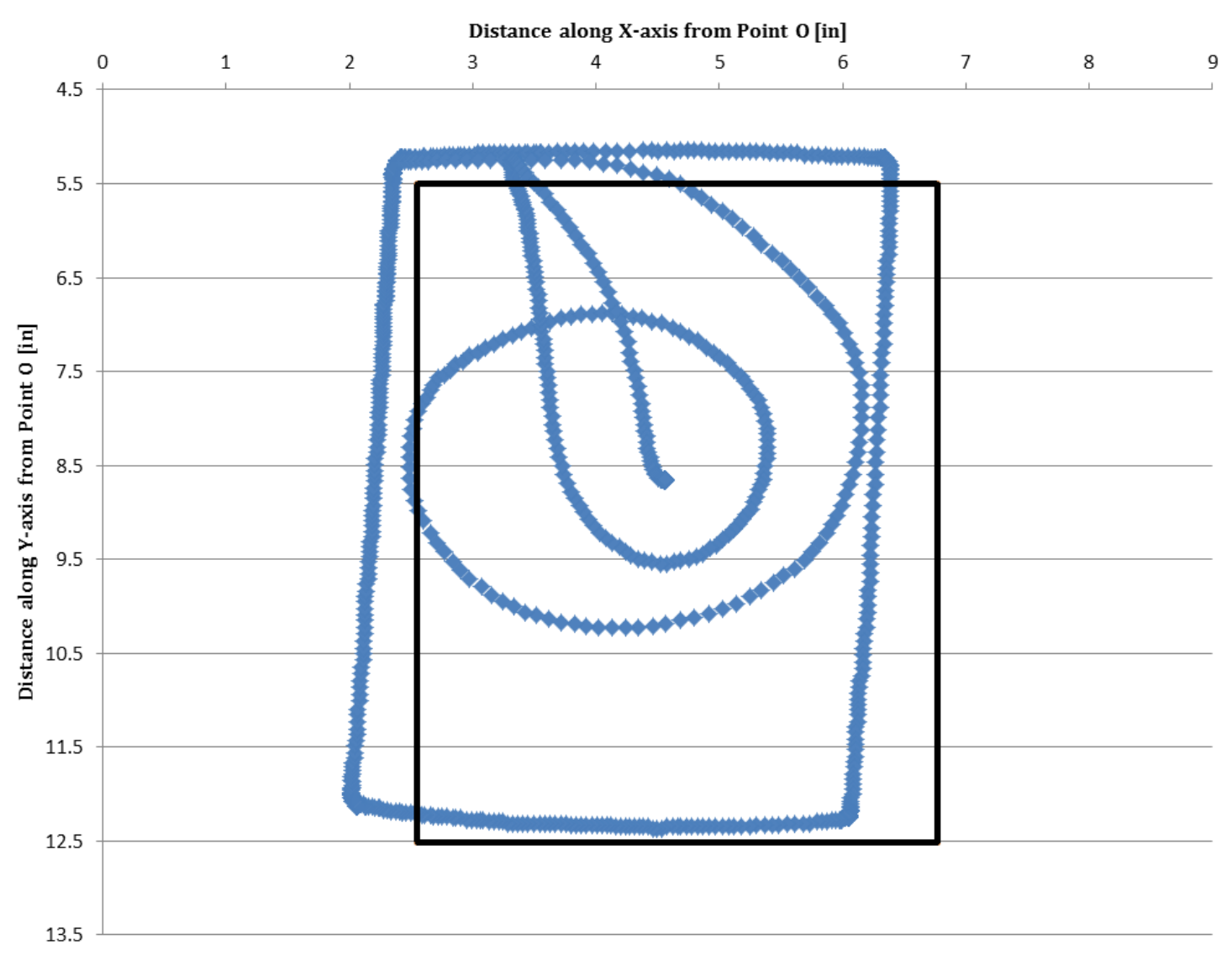

Figure 3.11: Validation test results showing the discrepancy between the calculated control positions (locations of Point $\mathrm{C}$ ) and the actual location of the rectangular perimeter of the range of the control column. 
system geometry had proven to be quite difficult to survey due to the location of the control column in a confined space between the bulkheads and thus these measurements were tuned by iteratively implementing small changes (one the scale of $1 \mathrm{~cm}$ or less) to the input geometry until the discrepancies were resolved. The results of the tuned validation data can be seen in Figure 3.12 .

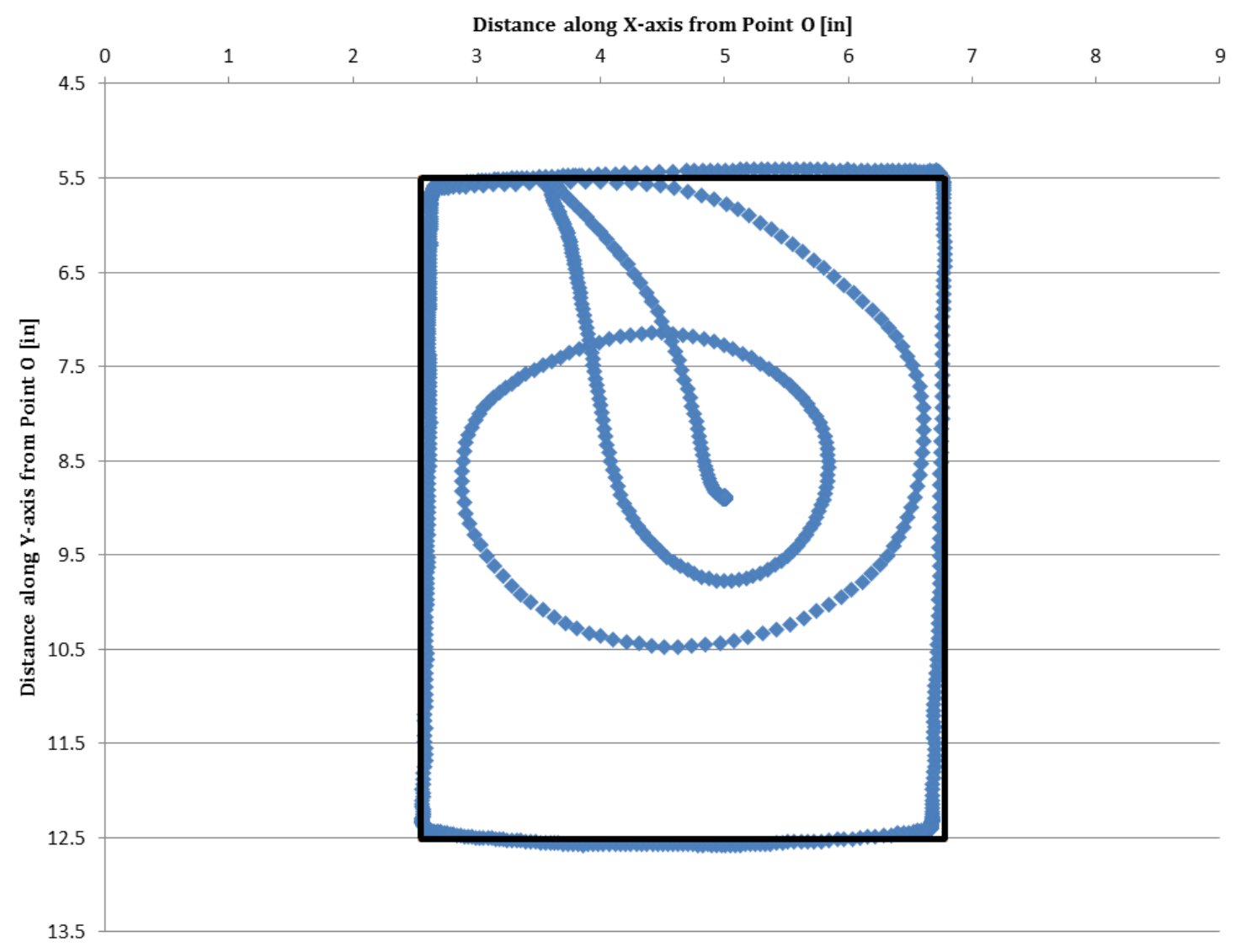

Figure 3.12: Validation test results using tuned geometry data showing the alignment of the calculated data points (locations of Point $\mathrm{C}$ ) with the rectangular perimeter of the range of the control column.

\subsubsection{Issues Encountered During Post-processing}

Following the flight tests, the data were scrutinized closely and two data collection issues were discovered. 


\section{GPS Data}

GPS data was not correctly measured and/or recorded during the first flight. Pre-flight testing had shown that the GPS had achieved a satellite fix and was recording what appeared to be sensible data; however, throughout the flight, those coordinates did not change as the location of the aircraft did. While the GPS data provides a convenient record of the absolute measurement of aircraft position, the two IMUs collected sufficient data to provide an additional means to generate trajectories of specific manoeuvres using well-established integration methods. As the GPS recorded proper position data during the second flight, the integration methods could be validated by comparing the calculated trajectories with those from the GPS data.

\section{Airspeed Data}

The second issue was the discovery that the airspeed sensor had not recorded any sensible data for either flight. The sensor had been checked pre-flight with the aircraft sitting still and it had been recording the expected near-zero numbers with very small fluctuations. During flight it continued to read near zero. This was unfortunately not noticed in the inspection of data between flights, particularly because the first few minutes of data showed an airspeed of up to 12 knots, which could have been expected during taxi and run-up. For the remainder of the flight, the airspeed sensor recorded numbers of the order $10^{-10}$ to $10^{-45}$. Similar values were recorded throughout the second flight.

It was hypothesized that the leak check and pressure test performed by the AME after integrating the sensor into the pitot-static system had surpassed the capabilities of the sensor and rendered it incapable of providing correct readings. Testing the sensor with a manometer after flight testing showed this was not the issue as the airspeed sensor output accurate readings during the manometer test. Some issues were found with the wiring; when the airspeed sensor was connected to the APM for testing, several failed 
readings were taken but quickly fixed by unplugging and plugging in the system once again, demonstrating potential reliability issues with the sensor wiring.

It was this type of issue which proved the merit of the visual recording of the flight. The GoPro video has a clear recording of the instrument panel. While the resolution of the video is not high, the airspeed indicator has a white needle and a black faceplate which makes it possible to use image processing software to track the needle orientation and calculate the corresponding airspeed. This solution does introduce error into the system due to instrument error (airspeed indicators are calibrated for standard atmospheric conditions at sea level), and instrument lag. There is also potential for precision errors to be introduced by image processing software. It is, however, a reasonable approach and the best possible approach as performing additional flight testing is not feasible at this time.

\section{Control Input Calibration}

The data from the string potentiometers was found to have very short, periodic errors in the data where all three string potentiometer readings dropped to impossibly small values. These errors occurred regularly, once every 4000 data points (approximately once every minute and a half) and lasted approximately $1 / 10$ th of a second each time. These missing points were interpolated with no anticipated adverse effect on the accuracy of the data.

\subsection{Discussion}

The flight testing methodology was devised as a minimally-intrusive way of acquiring performance data for a small aircraft like the Katana. The test equipment consisted mostly of self-contained systems which recorded flight control inputs and the aircraft response to these inputs. The equipment set-up could easily be transfered to other small aircraft to perform similar testing. 
The flight testing can be considered a success but for the lack of airspeed data, though the video record of the flight does provide an acceptable solution. Despite the issues encountered in post-processing, the data acquired is more than adequate to evaluate selected data points as discussed in Chapter 4. More robust and reliable GPS and airspeed measurement systems should be investigated and incorporated into the system for any future testing initiatives. Additionally, a method for monitoring the recorded data in flight, such as a small tablet connected to the laptop, would greatly reduce the risk of these errors going unnoticed during future flights. 


\section{Chapter 4}

\section{Flight Model}

\subsection{Plane Maker}

The flight model for the KatanaSim was created using Plane Maker, part of the X-Plane software package from Laminar Research in Columbia, South Carolina [27]. Plane Maker allows users to design or modify aircraft models for use in X-Plane. Physical specifications of the desired aircraft are entered into the program and assembled into an aircraft (.acf) file. This file is opened in X-Plane which uses these specifications to determine the appearance of the aircraft, and more importantly, predict how the aircraft will perform in flight. Much of the information that can be input to Plane Maker has no effect on the final flight model as it pertains to information such as cabin lighting, electrical wiring, cockpit appearance, and special add-ons such as parachutes, weapons, or cargo drop capabilities. The parameters that do affect the flight model are geometric descriptions of the aircraft and engine specifications. It should be noted that no explicit performance parameters can be specified; the user is completely reliant on X-Plane for the determination of the flight model. A screenshot of the Plane Maker user interface is shown in Figure 4.1. 


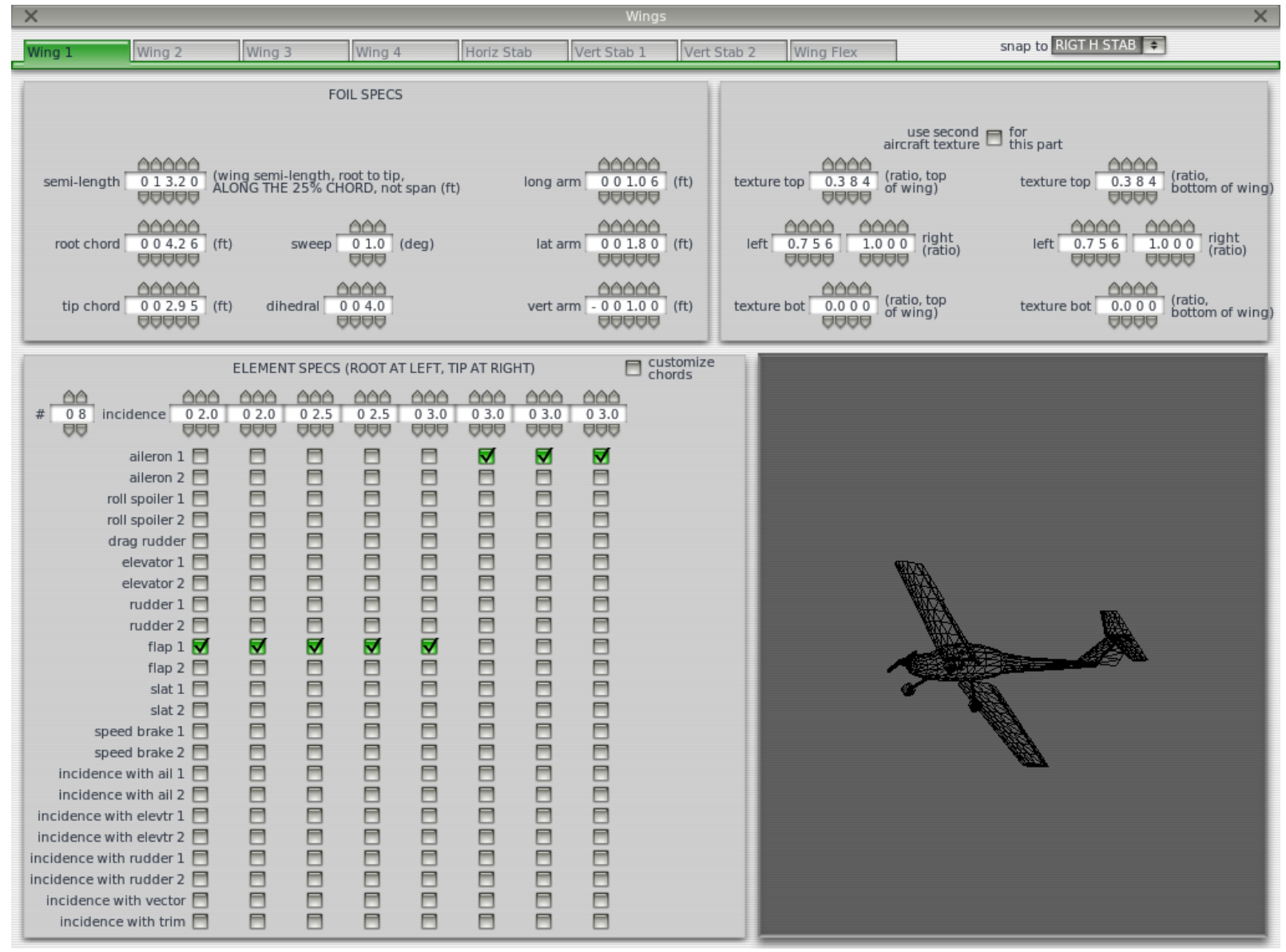

Figure 4.1: A screenshot of Plane Maker showing the user interface for defining the lifting surfaces of an aircraft. 


\subsubsection{Blade Element Theory}

The flight dynamics of the aircraft are calculated in X-Plane using information from Plane Maker and employing blade element theory (BET). The basic steps of blade element theory entail discretizing of the lifting surfaces of the aircraft into small pieces, called elements. The forces acting on each of these elements are determined and summed up, and then used to find the aircraft translational and angular accelerations. These accelerations are then numerically integrated to find the aircraft velocity and position. These calculations are performed at least fifteen times per second in X-Plane [28]. BET is summarized below; a more detailed explanation can be found in [28].

The element breakdown is user defined in Plane Maker and applies to the wings, horizontal and vertical stabilizers, and propeller. A larger number of elements will increase the accuracy of the roll rates and accelerations being calculated, but also the required computation time. The makers of X-Plane determined that ten elements per side per lifting surface would be the maximum allowable number of elements as ten would generate results sufficiently close to those results obtained using much higher numbers of elements.

In determining the velocity vector of each element, X-Plane takes many factors into consideration. Prop wash is calculated by applying the principles of conservation of momentum to the propeller disc and resulting thrust. Downwash is calculated by looking at the aspect ratio, taper ratio, sweep angle, and location of the horizontal stabilizer with respect to the wing. X-Plane has an empirical look-up table which is used to find the degree of downwash generated per lift coefficient. The velocity vectors are found using the linear and angular velocities and locations of each element and considering the effects of downwash, prop wash, and induced angle of attack (if flaps have been deployed). The velocity vectors are then used to calculate the dynamic pressures for each element.

A two-dimensional airfoil profile is entered into Plane Maker to which X-Plane applies finite wing property reductions to the lift slope, maximum lift coefficient, induced drag, 
and moment for the specified aspect ratio, taper ratio, and sweep of the particular lifting surface. At this stage, any compressible flow effects in X-Plane would be considered using the Prandtl-Glauert method; however, even at the maximum allowable airspeed of $298 \mathrm{kph}$ (161 knots) at the service ceiling of $4000 \mathrm{~m}$ (13,121 feet) above mean sea level [16], the Katana only reaches Mach 0.25. Compressible flow effects can be ignored for this particular flight model as they are typically considered negligible under Mach 0.3 [35].

Using the previously-determined element areas, dynamic pressures, and aerodynamic coefficients, the forces on each element are found and summed for all surfaces. The total force is then used to determine linear accelerations and angular accelerations of the aircraft. These accelerations are then integrated to find the velocity, position, and orientation of the aircraft.

This method of calculating the flight model has the potential to be extremely accurate; as mentioned in Section 2.12, multiple flight simulators using X-Plane and Plane Makerdeveloped flight models have been certified in North America.

\section{Advantages and Disadvantages}

Most flight simulator software packages on the market, most notably Microsoft Flight Simulator (MSFS), use stability derivatives (SDs) to define their flight models, unlike the use of BET with X-Plane. The greatest difference between BET and SDs is that the BET is able to predict the behaviour of an aircraft, while SDs can only be used to define the behaviour of the aircraft. While X-Plane can be used to test the behaviour of any sort of aircraft the user could imagine and create in Plane Maker, MSFS aircraft must be given predefined flight models which can be particularly hard to obtain for military or experimental aircraft.

Stability derivatives, discussed in detail in Chapter 5, approximate the aircraft as a whole rather than calculate the effect of each lifting surface as does BET. Because of 
this, SDs cannot adequately model asymmetric engine failures on multi-engine aircraft or dynamic effects generated by propellers and rotors such as spiraling slipstreams, P-factor, and translational lift. The dynamics of the aircraft are also calculated with the assumption that the body of the aircraft is symmetric and that the centre of gravity and moment of inertia are constant throughout the flight. With respect to the KatanaSim, BET offers much more realistic performance for irregular and edge-of-envelope manoeuvres such as stalls, spins, and periods of significant turbulence.

Overall, BET is a more robust and accurate flight modelling tool for a wider variety of flight conditions. The particular disadvantage of X-Plane's use of BET is the lack of the ability to easily tune the flight model for the KatanaSim. Flight model tuning with software like MSFS would consist of simply changing performance parameters in an input file to match the desired performance characteristics of the aircraft. By contrast, model tuning in Plane Maker is limited to making strategic changes to the geometry and power specifications targeted at effecting the desired performance changes.

\subsection{A Katana Flight Model}

While X-Plane releases their software with a few dozen aircraft models included, the Katana is not one of them. Additional aircraft models, including Katanas, are available through the extensive X-Plane online community, but with no guarantees available as to the pedigrees of the models as they are created by hobbyists with varying levels of experience in aviation. The most popular online Katana models were built by hobbyists who had completed, or were in the process of completing, PPL training on Katanas at their local flying clubs. They used information available from the Katana Pilot Operating Handbook (POH), and then simply used their best judgment as to whether or not their flight models performed accurately and adjusted parameters until they were satisfied. 
The KatanaSim required a flight model accurate enough to avoid students experiencing any negative training transfer. Because the publicly-available Katana models lacked a full pedigree, a flight model was built from scratch and each data point was documented in order to ensure the greatest accuracy and traceability possible. The information used to build the KatanaSim flight model was sourced wherever possible from published sources with the remaining data found through empirical measurements and observations.

\subsubsection{Katana Specification Research}

Published data was gathered from multiple sources in an attempt to define as many dimensions and performance specifications for the Katana as possible. Sources from Diamond Aircraft Industries included the POH, and aircraft maintenance manual (AMM), and illustrated parts catalog (IPC). Additional information was found in the Transport Canada (TC) and Federal Aviation Authority (FAA) type certification data sheets (TCDS). Information regarding aircraft geometry that could not be gleaned from the published data was found by physical inspection of the aircraft components.

The Wortmann FX63-137/20 airfoil used on the Katana does not have any published information as it is a proprietary airfoil of Diamond Aircraft Industries. In order to acquire the geometric data required to create an airfoil file for use with Plane Maker, the wing of a Katana was measured using the Optotrak Coordinate Measurement Machine shown in Figure 4.2. Ninety points were measured along the upper and lower surfaces of a wing cross section, with a higher density of points being measured along the leading and trailing edges. The resulting airfoil profile appears in Figure 4.3.

These coordinates were then analysed using JavaFoil [36], an airfoil analysis program capable of creating airfoil files compatible with Plane Maker. These files contain information on the geometry and aerodynamic behaviour of the aircraft at a given Reynolds number. As JavaFoil is a notoriously complex program, advice was sought from the on-line 

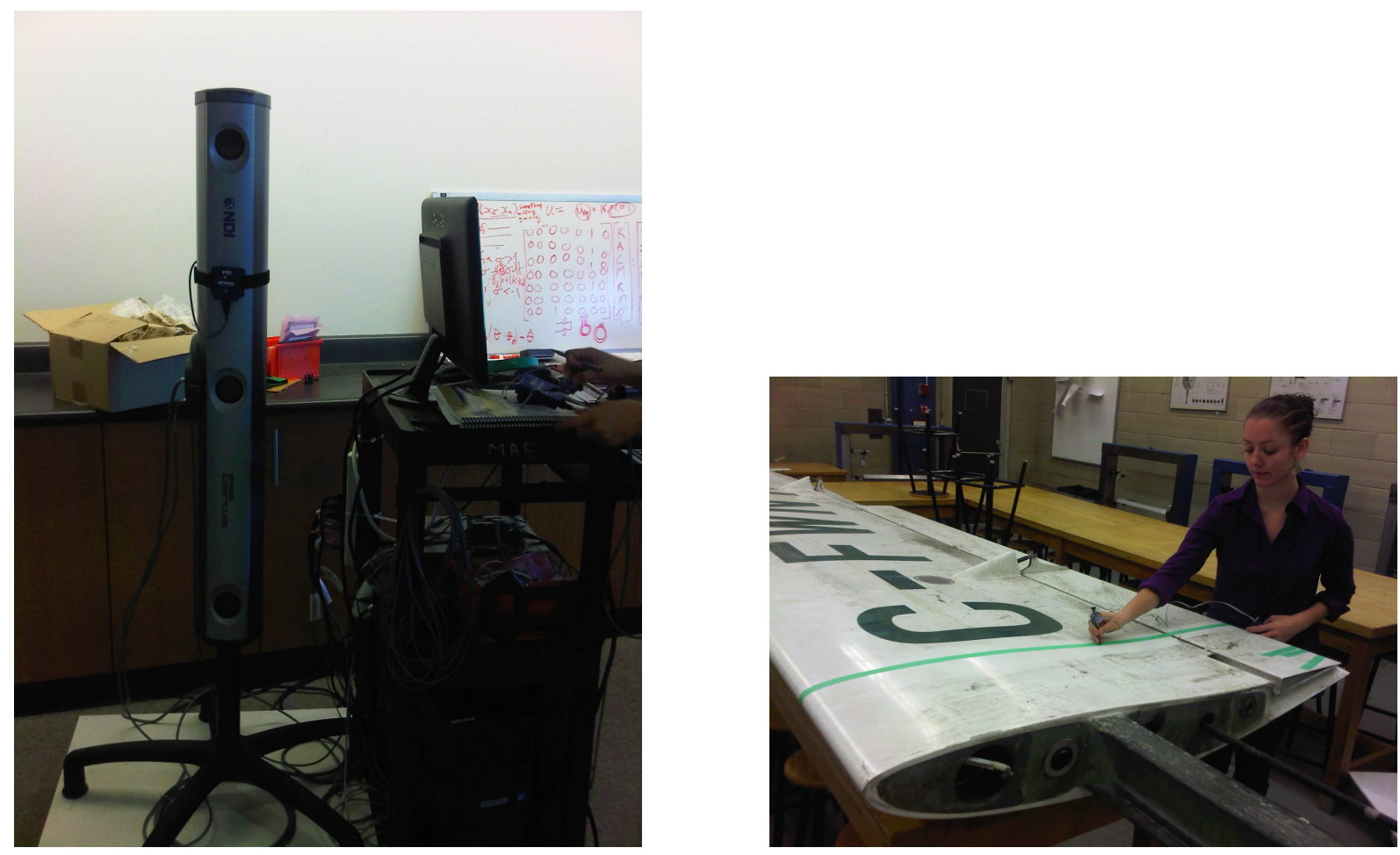

Figure 4.2: Optotrak sensor tower (left) and stylus being used to measure the airfoil coordinates of a Katana wing (right).

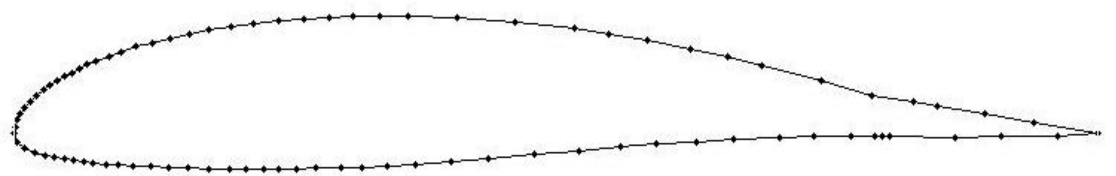

Figure 4.3: Wortmann FX63-137/20 airfoil profile as measured using the Optotrak Coordinate Measurement Machine.

X-Plane community. Dr. Bernd Sieker of the Faculty of Technology at the University of Bielefeld in Germany offered to help. Dr. Sieker is an aviation incident analyst, and private pilot with extensive experience in X-Plane and Plane maker. He offered to create and share airfoil files using the FX63-137/20 coordinates provided to him. As Plane Maker allows for two airfoil files of different Reynolds number to be uploaded, Dr. Sieker suggested using Reynolds numbers of 4.5 million and 1 million for the Katana and provided files for each. Airfoil Maker was used to view the resulting polars for the airfoil; these can be seen in Figure 4.4. The curves for the coefficients of aerodynamic lift, drag, and pitching 


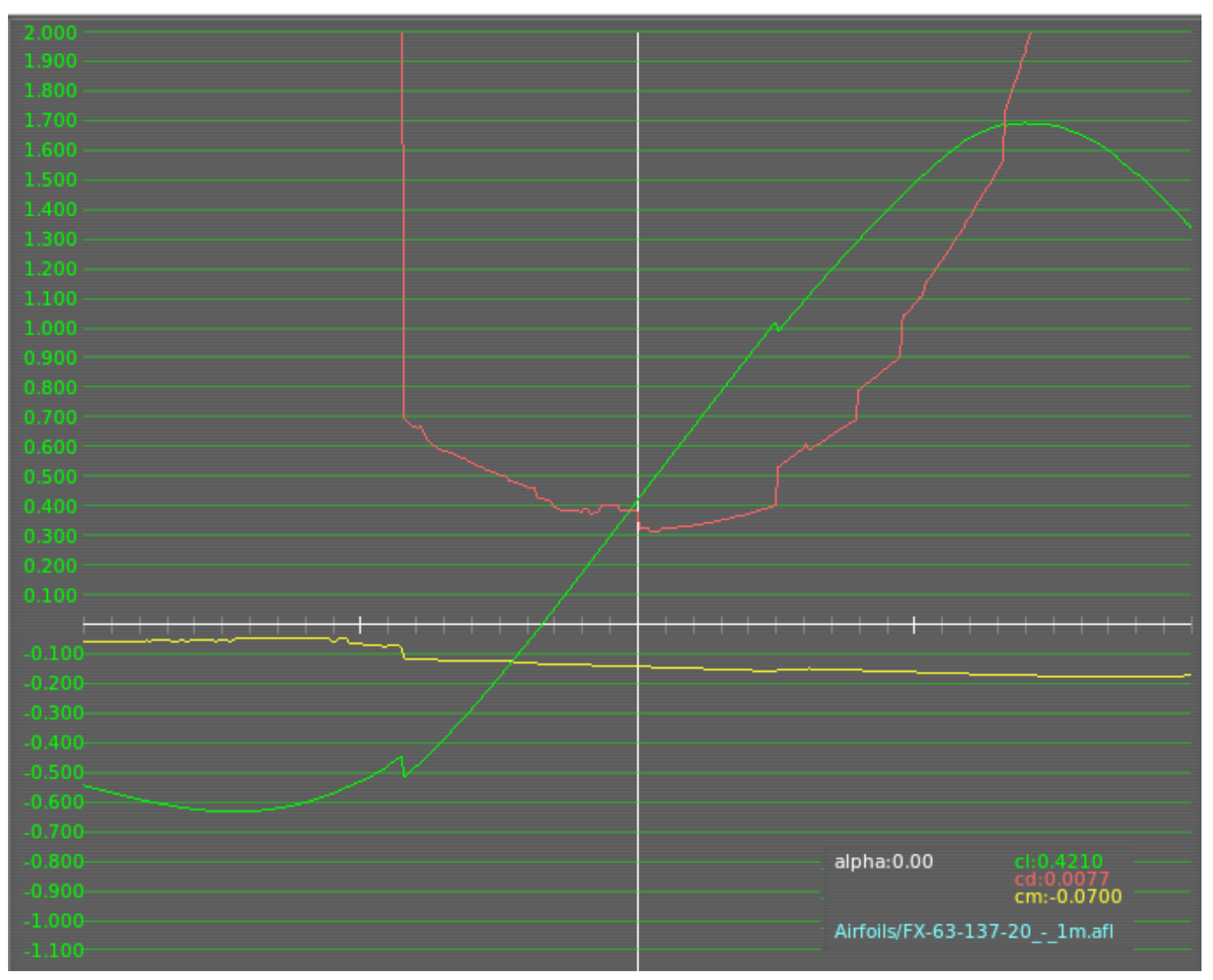

Figure 4.4: Airfoil Maker polars for the Wortmann FX63-137/20 airfoil showing the coefficients of aerodynamic lift, drag, and pitching moment for angles of attack from $-20^{\circ}$ to $20^{\circ}$, where each tick represents $1^{\circ}$ and the vertical axis indicates a zero angle of attack.

moment are shown for angles of attack from $-20^{\circ}$ to $20^{\circ}$. A distinct drag bucket can be seen between $0^{\circ}$ and $5^{\circ}$, indicating increased laminar flow and the subsequent decrease in skin friction drag for this range.

\subsubsection{Building the Flight Model}

The published data and empirical measurements were used to create the flight model; however, several challenges were encountered as inconsistencies were found in the published data when compared to the measurements taken. In Phase One of the flight model creation, the published geometry data was entered into Plane Maker, and the 3-view drawings provided in the AMM and IPC were used to model the fuselage, wings, landing gear, and 
control surfaces. Immediately some issues were encountered as the 3-view drawings were not consistent in dimensioning the wing span of the aircraft.

Empirical measurements were used to fill in any required dimensions in Plane Maker which were not provided on the 3-view diagrams, and to reconcile the inconsistencies in published data. The resulting model was not unlike the Katana, but it was immediately obvious that there were differences in the geometry of the model from that of the actual aircraft.

When the model was compared with photographs showing the front and side profiles of the Katana, it became clear that the 3-view drawings were incorrect in depicting the shape of the fuselage, the location and size of the empennage, and the orientation of the landing gear. Plane maker allows for photographs to be loaded as a background image to aid in modelling the fuselage contour. Rather than use the 3-view drawings as had been done in the first phase, the photographs of the aircraft were used in the second phase to fine tune the geometry. The contours of the aircraft changed significantly with the fuselage being made wider and shorter, and being given a flatter base. The wings were given a lower attachment point on the fuselage and the landing gear was lengthened. The sweep angle of the vertical stabilizer was lessened and the horizontal stabilizer was lowered. While the parameters required to achieve the new model differed somewhat from the published data, the appearance of the resulting model was markedly more accurate.

In the third phase of the model creation, the airfoil was loaded and the performance information for the powerplant (found in the POH and on the TCDSs) was entered. The weight and balance of the aircraft was also specified. As X-Plane requires an aircraft model to have an instrument panel, despite the fact that the panel would never be used in the KatanaSim due to the availability of a functional physical instrument panel, an instrument panel file from the Cessna 172S (created by Laminar Research) was added to the model. Similarly, other parameters such as electrical systems, aircraft paint scheme, and cockpit 
lighting were neglected as they would have no effect on the flight model and would be of no use in the KatanaSim.

The maiden flight of the resulting Katana model was successful in that it achieved flight and was easy to control; however, it was clear that it was not an accurate flight model. During training, students are taught to perform instrument scans and to ensure that certain performance criteria such as engine RPM, aircraft attitude, airspeed, and vertical speed are obtained. These performance points could also be found from analysis of the flight test data. Although some variations in performance can be expected, due to the effects of weather and aircraft eccentricities, the initial flight model deviated from the expected and desired performance. The engine power was much greater than ever achieved in a real Katana; the aircraft model idled with a higher RPM, flew faster, climbed faster, and was unable to achieve a power-off stall unless the elevator was abruptly deflected.

\subsection{Model Evaluation}

As X-plane calculates the flight model based on the input parameters, model tuning would require either an in-depth understanding of the blade element modelling methods of XPlane, or an exploration of Plane Maker parameters and their effect on the flight dynamics of the model in an attempt to reverse engineer the system. As neither the X-Plane manual nor the Plane Maker manual provided any insight into model tuning, the latter option for tuning was chosen. The feasibility of manually identifying the effect of Plane Maker parameters on the model performance was investigated using a sensitivity study. The study was deliberately not all-encompassing as it served only to determine if the method in question was a feasible option for tuning the KatanaSim.

Three flight scenarios were investigated: climb with take-off configuration, straight and level flight, and stall. For each scenario, four performance points were recorded: 
propeller speed, air speed, climb rate, and pitch angle. A sensitivity study was performed to investigate the effects of variations in the centre of gravity $(\mathrm{CG})$ location, the wing span, and the maximum available power settings with respect to the chosen performance points. Each parameter was varied eight times for each flight situation.

The performance graphs can be found in Appendix F. Plane Maker parameters are defined using Imperial units, and aircraft flight gauges (actual and X-Plane simulated gauges) also use Imperial units with certain standard aviation conventions such as feet per minute for climb rates and inches of mercury for manifold pressure measurements. For the remainder of this section, Plane Maker parameters and performance points will be discussed using the conventions used in X-Plane, Plane Maker and in general aviation.

\subsubsection{Climb with Take-off Configuration}

After take-off, a proper climb-out requires full throttle and maximum propeller RPM with take-off flaps deployed, and an attitude chosen to achieve an airspeed of 65 knots. From the second flight test, the average recorded pitch angle during climb-out was calculated to be $6.3^{\circ}$ and the average climb rate to be $560 \mathrm{ft} / \mathrm{min}$. However, mild turbulence during the flight test made a steady airspeed impossible to achieve; the airspeed often wavered between 60 and 65 knots which suggested the pitch angle was slightly lower than the ideal. It can also be noted that the cold weather would have resulted in climb rates higher than those attainable in warmer seasons due to the increased density of the cold air and the resulting increase in lift.

The flight model, before any changes were made to the model for the sensitivity study, achieved surprisingly high climb rates of $700 \mathrm{ft} / \mathrm{min}$ or more with high pitch angles of $8-10^{\circ}$. 


\section{Variations in Longitudinal Placement of the CG}

For the sensitivity study, the CG was varied 1 inch at a time from 4 inches forward of the neutral point, to 3 inches aft of it. The resulting pitch angles and climb rates showed no significant correlation with the CG location, although it was noted that the control inputs required to achieve the climb varied due to the change in pitching moment of the aircraft. Recorded performance data can be seen in Table 4.1.

Table 4.1: Effect of CG location on take-off climb performance.

\begin{tabular}{ccccc}
\hline $\begin{array}{c}\text { CG location } \\
\text { [in] }\end{array}$ & $\begin{array}{c}\text { Airspeed } \\
{[\mathrm{knots}]}\end{array}$ & $\begin{array}{c}\text { Pitch } \\
{[\mathrm{deg}]}\end{array}$ & $\begin{array}{c}\text { Climb } \\
{[\mathrm{ft} / \mathrm{min}]}\end{array}$ & $\begin{array}{c}\text { Prop. speed } \\
{[\mathrm{RPM}]}\end{array}$ \\
\hline-4 & 65 & 9.3 & 683 & 2391 \\
-3 & 65 & 9.8 & 725 & 2383 \\
-2 & 65 & 9.7 & 720 & 2383 \\
-1 & 65 & 9.8 & 740 & 2383 \\
0 & 65 & 9.7 & 738 & 2383 \\
1 & 65 & 9.7 & 735 & 2383 \\
2 & 65 & 9.3 & 700 & 2383 \\
3 & 65 & 9.6 & 749 & 2383 \\
\hline
\end{tabular}

\section{Variations in Wing Span}

The semi-length of the wing span was tested in 0.5 foot increments from 11.7 to 15.2 feet. The increase in wing span was directly correlated with a decrease in pitch angle but also an increase in climb rate. The lowest pitch angle observed was $7.4^{\circ}$ with a 15.2 foot wing semi-length and a subsequent climb rate of $701 \mathrm{ft} / \mathrm{min}$. This pitch angle was much closer to the flight test value suggesting an increase in the wing span of the model might be beneficial. Unfortunately, because of the inverse relationship with the climb rate, a tradeoff would be required between the accuracy of the pitch angle and that of the climb rate. Recorded performance data can be seen in Table 4.2. 
Table 4.2: Effect of wing span on take-off climb performance.

\begin{tabular}{ccccc}
\hline $\begin{array}{c}\text { Wing semi length } \\
{[\mathrm{deg}]}\end{array}$ & $\begin{array}{c}\text { Airspeed } \\
{[\mathrm{knots}]}\end{array}$ & $\begin{array}{c}\text { Pitch } \\
{[\mathrm{deg}]}\end{array}$ & $\begin{array}{c}\text { Climb } \\
{[\mathrm{ft} / \mathrm{min}]}\end{array}$ & $\begin{array}{c}\text { Prop. speed } \\
{[\mathrm{RPM}]}\end{array}$ \\
\hline 11.7 & 65 & 10 & 631 & 2118 \\
12.2 & 65 & 9.3 & 645 & 2375 \\
12.7 & 65 & 9.2 & 688 & 2383 \\
13.2 & 65 & 8.8 & 675 & 2384 \\
13.7 & 65 & 8.7 & 666 & 2390 \\
14.2 & 65 & 8.6 & 745 & 2383 \\
14.7 & 65 & 8.4 & 723 & 2384 \\
15.2 & 65 & 7.4 & 701 & 2376 \\
\hline
\end{tabular}

\section{Variations in Maximum Available Power}

In every Katana aircraft there is an 80 HP Rotax 912 engine. The performance of the flight model was tested with maximum available power ratings set between 60 and 88 HP. Both pitch and climb rate were, as expected, directly correlated with the power rating. On the lowest end of the test spectrum, a $60 \mathrm{HP}$ engine had a pitch angle of just $6.4^{\circ}$ and a climb rate of $367 \mathrm{ft} / \mathrm{min}$. This pitch angle was the best approximation of the average pitch angle in the take-off climb during flight testing. The climb rate was also the lowest achieved and was in fact lower than the flight test data, though perhaps this could be considered even more accurate as the X-Plane simulated weather during testing was not as cold as the actual weather during flight testing, which would lead to a lower climb rate. Recorded performance data can be seen in Table 4.3.

\subsubsection{Straight and Level Flight}

The propeller speed and manifold pressure in the flight model were set to approximately $2400 \mathrm{RPM}$ and 24 in $\mathrm{Hg}$ respectively, as these are the manufacturer-recommended cruise settings for the Katana aircraft; these settings typically result in a 90 knot trimmed cruising 
Table 4.3: Effect of maximum available engine power on take-off climb flight performance.

\begin{tabular}{ccccc}
\hline $\begin{array}{c}\text { Max. power } \\
{[\mathrm{HP}]}\end{array}$ & $\begin{array}{c}\text { Airspeed } \\
{[\mathrm{knots}]}\end{array}$ & $\begin{array}{c}\text { Pitch } \\
{[\mathrm{deg}]}\end{array}$ & $\begin{array}{c}\text { Climb } \\
{[\mathrm{ft} / \mathrm{min}]}\end{array}$ & $\begin{array}{c}\text { Prop. speed } \\
{[\mathrm{RPM}]}\end{array}$ \\
\hline 60 & 65 & 6.4 & 367 & 2178 \\
64 & 65 & 6.6 & 389 & 2231 \\
68 & 65 & 7.9 & 537 & 2270 \\
72 & 65 & 8.1 & 563 & 2311 \\
76 & 65 & 8.4 & 626 & 2348 \\
80 & 65 & 8.8 & 664 & 2386 \\
84 & 65 & 9.8 & 746 & 2420 \\
15.2 & 65 & 9.5 & 747 & 2435 \\
\hline
\end{tabular}

speed. From the fight test data, it was determined that the average pitch angle during straight and level flight was $-1.19^{\circ}$. The autopilot in the KatanaSim was instructed to maintain straight and level flight for each flight model given a throttle setting of 2400 RPM and a manifold pressure of 24 in $\mathrm{Hg}$.

\section{Variations in Longitudinal Placement of the CG}

As was observed with the take-off climb portion of the sensitivity study, the variation in longitudinal placement of the CG had no observable effect on the airspeed of the flight model which was consistent at 115 knots, significantly higher than the desired 90 knot speed. The incremental changes in CG location did have an extremely small effect $\left(<-0.05^{\circ}\right.$ per 1 inch of CG location change) on the pitch angle. The pitch angle of the CG in the centre of the allowed envelope was $-1.05^{\circ}$ which was very close to the measured flight test value. Recorded performance data can be seen in Table 4.4.

\section{Variations in Wing Span}

An increase in wing span corresponded to a decrease in cruising airspeed though scatter in the data made it difficult to determine the exact relationship. The small decreases in 
Table 4.4: Effect of CG location on straight and level flight performance.

\begin{tabular}{ccccc}
\hline $\begin{array}{c}\text { CG location } \\
\text { [in] }\end{array}$ & $\begin{array}{c}\text { Airspeed } \\
{[\mathrm{knots}]}\end{array}$ & $\begin{array}{c}\text { Pitch } \\
{[\mathrm{deg}]}\end{array}$ & $\begin{array}{c}\text { Climb } \\
{[\mathrm{ft} / \mathrm{min}]}\end{array}$ & $\begin{array}{c}\text { Prop. speed } \\
{[\mathrm{RPM}]}\end{array}$ \\
\hline-4 & 115.6 & -0.995 & 0 & 2334 \\
-3 & 114.9 & -0.984 & 0 & 2397 \\
-2 & 115.2 & -1.035 & 0 & 2366 \\
-1 & 115.0 & -1.034 & 0 & 2428 \\
0 & 115.0 & -1.051 & 0 & 2397 \\
1 & 115.2 & -1.081 & 0 & 2397 \\
2 & 115.2 & -1.102 & 0 & 2397 \\
3 & 115.0 & -1.119 & 0 & 2428 \\
\hline
\end{tabular}

cruising airspeed with each wing span increase of 1 foot would not be sufficient to enable the cruise speed of the flight model to be reduced by the required 20-30 knots. Since both the cruise airspeed and pitch angle trended towards values that more closely matched the flight test data, it suggests that a slight increase in wing span of the model could be one of several changes that would be required to fully tune the model. Recorded performance data can be seen in Table 4.5.

Table 4.5: Effect of wing span on straight and level flight performance.

\begin{tabular}{ccccc}
\hline $\begin{array}{c}\text { Wing semi length } \\
{[\mathrm{deg}]}\end{array}$ & $\begin{array}{c}\text { Airspeed } \\
{[\mathrm{knots}]}\end{array}$ & $\begin{array}{c}\text { Pitch } \\
{[\mathrm{deg}]}\end{array}$ & $\begin{array}{c}\text { Climb } \\
{[\mathrm{ft} / \mathrm{min}]}\end{array}$ & $\begin{array}{c}\text { Prop. speed } \\
{[\mathrm{RPM}]}\end{array}$ \\
\hline 11.7 & 119.4 & -0.805 & 0 & 2397 \\
12.2 & 113.0 & -0.568 & 0 & 2366 \\
12.7 & 116.6 & -0.900 & 0 & 2366 \\
13.2 & 112.9 & -0.900 & 0 & 2334 \\
13.7 & 114.1 & -1.131 & 0 & 2366 \\
14.2 & 111.4 & -1.100 & 0 & 2397 \\
14.7 & 112.5 & -1.313 & 0 & 2366 \\
15.2 & 111.5 & -1.249 & 0 & 2366 \\
\hline
\end{tabular}




\section{Variations in Maximum Available Power}

The change in maximum available power from $64 \mathrm{HP}$ to $88 \mathrm{HP}$ produced significant changes in the pitch angle and cruise speed of the aircraft in straight and level flight. As the power was increased, the cruise speed increased from 98 knots (64 HP) to 121 knots ( $88 \mathrm{HP}$ ). The pitch angle decreased from $0.299^{\circ}(64 \mathrm{HP})$ to $-1.399^{\circ}$ ( $88 \mathrm{HP}$ ). Of the tested parameters, available power had the greatest effect on the airspeed. As with the take-off climb portion of the sensitivity study, it was concluded that a decrease in power would increase the accuracy of the flight model. Recorded performance data can be seen in Table 4.6.

Table 4.6: Effect of maximum available engine power on straight and level flight performance.

\begin{tabular}{ccccc}
\hline $\begin{array}{c}\text { Max. power } \\
{[\mathrm{HP}]}\end{array}$ & $\begin{array}{c}\text { Airspeed } \\
{[\mathrm{knots}]}\end{array}$ & $\begin{array}{c}\text { Pitch } \\
{[\mathrm{deg}]}\end{array}$ & $\begin{array}{c}\text { Climb } \\
{[\mathrm{ft} / \mathrm{min}]}\end{array}$ & $\begin{array}{c}\text { Prop. speed } \\
{[\mathrm{RPM}]}\end{array}$ \\
\hline 60 & 98.1 & 0.299 & 0 & 2376 \\
64 & 102.3 & -0.092 & 0 & 2441 \\
68 & 106.6 & -0.447 & 0 & 2532 \\
72 & 111.9 & -0.834 & 0 & 2552 \\
76 & 114.4 & -1.002 & 0 & 2366 \\
80 & 115.0 & -1.039 & 0 & 2397 \\
84 & 118.2 & -1.220 & 0 & 2366 \\
88 & 121.3 & -1.399 & 0 & 2397 \\
\hline
\end{tabular}

\subsubsection{Stalls}

When the original flight model was tested in the KatanaSim, it was discovered that the aircraft would not stall when the throttle was brought to idle and the control column was gently brought to the full pitch up position. During the stalls initiated during the flight tests, the warning horn would sound when the airspeed was between 50 and 55 knots, and the nose of the aircraft would drop abruptly when the airspeed was between 35 and 40 knots. These conditions and control inputs were mimicked in the KatanaSim but the 
response of the aircraft could not be reproduced and all of the KatanaSim flight models that were tested produced the same results. In the KatanaSim, the aircraft model would slow to an airspeed between 50 and 55 knots, achieved with full pitch up control column inputs and throttle at idle. The stall warning would not sound and no further airspeed would be lost. The nose would drop slightly, though not as abruptly as during a stall, and the climb rate would decrease sharply until the throttle was advanced and pitch inputs were added.

No improvements could be made to the stalling characteristics of the KatanaSim flight model based on the implemented sensitivity study.

\subsection{Discussion}

The creation of a Katana flight model using only published documentation would be impossible due to inconsistencies in the existing data, and the lack of comprehensive performance data. Obtaining the rest of the required information was possible due to empirical measurements taken by members of the ADL and due to the design and implementation of a minimally-intrusive flight test method. The empirically-gathered data did not always agree with the published data; however, the end result of integrating the gathered data in the flight model produced noticeably more accurate results.

It has become abundantly clear that the X-Plane software package has both advantages and disadvantages when it is used for the development of a cost-effective small aircraft simulator. The software is commercially available and comes complete with a flight model engine, advanced weather-simulating capabilities, modifiable scenery, and the ability to build and edit aircraft models. The use of blade element theory for predicting aircraft performance produces better results than would stability derivatives, but at the cost of being able to easily and efficiently tune the performance of a given model. 
The sensitivity study detailed one possible method of tuning the flight model by identifying the effects of each Plane Maker parameter on a given set of performance points. While this method may eventually yield sufficient results to tune the flight model, it is extremely time-consuming, can produce conflicting results (e.g., an increased wingspan improves pitch angle accuracy but decreases climb rate accuracy), and there is no guarantee that the desired performance points will be achieved. These challenges emphasized the need for a better tuning system that would not require manual iterations to be performed and would be able to tune multiple parameters simultaneously. No further tuning was attempted on the flight model and the project focus shifted on to the development of an automated tuning method instead. 


\section{Chapter 5}

\section{Automated Flight Model Tuning}

This chapter will review several of the most commonly used flight modelling methods and flight parameter estimation techniques. Ideally, one of these methods would be applied to the Plane Maker flight model for the KatanaSim in order to tune the geometric parameters and achieve realistic flight performance. Unfortunately, the lack of access to the bladeelement-theory-based flight dynamics model restricts the ability to do so at the present time. Instead, a focus is placed on a stability-derivative-based math model as this is a commonly used flight dynamics model for commercially-available flight simulation software. The chapter will then move on to discuss the development of a genetic algorithm and the evolutionary theory behind the algorithm. The genetic algorithm uses the derived math model to tune stability derivatives based on simulated flight test data. Results of this curve fitting optimization using the genetic algorithm will be presented.

\subsection{Proven Flight Modelling Methods}

There are two main approaches to flight modelling: model prediction and parameter estimation.

Model prediction, unlike parameter estimation, does not require the equations of mo- 
tion of the system to be known. Model prediction methods, like neural networks, use complex algorithms to predict model behaviour based on given sets of input and output data. Rather than optimizing parameters used to define the system which are then entered into the mathematical model of the system, model prediction replaces the mathematical model completely.

Parameter estimation usually requires the equations of motion of the system to be known and the parameters contributing to the dynamics of the system to be identified. Flight test information or the desired performance data is used to optimize the flight parameters in order to produce the desired system output. Methods vary in level of complexity and can be used to solve for parameters such as aerodynamic coefficients or stability derivatives. These parameters are then entered into the flight modelling mathematical model to produce the desired behaviour in the flight model. Some examples of parameter estimation techniques include the output error method, Kalman filters, and genetic algorithms.

\subsubsection{Output Error Method}

The output error method is a maximum likelihood estimation technique based on minimizing the quadratic error between simulated and actual system outputs. Nonlinear programming algorithms are used to solve a nonlinear least-squares problem in order to converge on the optimal system parameters [37].

In Lee and Yoon [38], flight test data for a small single-engine aircraft was processed using the output error parameter estimation method. The results of the estimation were validated through comparisons of flight test performance with the performance of the simulated aircraft; while they did not meet the requirements for a Level 7 FAA certified flight training device (the highest level attainable), they did meet the requirements for Level 5 certification. 
While the output error method has proven its value, the method does have weaknesses. The output error method will converge well on a local maximum but global convergence does not occur. More accurate results can be produced using prediction error methods or other algorithms which are better suited for global convergence, e.g., evolutionary algorithms [39].

\subsubsection{Kalman Filters}

Extended Kalman filters (EKFs) and unscented Kalman filters (UKFs) have both been used for recursive flight parameter estimation. Using filters for parameter estimation requires modelling the problem as a state estimation problem and then artificially defining the unknown parameters as state variables before solving. This inherently creates a nonlinear system, even if the original system was linear (though this is not the case for aircraft dynamics). EKFs work on the principle of applying instantaneous linearizations to each time step in order to approximate the nonlinearities. Strong nonlinearities in a system can cause difficulties with the EKF, leading to incorrect results or divergence. Despite this, EKFs are one of the most popular filtering techniques in the aerospace industry and have been implemented successfully in a variety of parameter estimation problems. UKFs overcome the inability of EKFs to handle nonlinear systems by avoiding the approximations introduced by the linearizations in the EKFs. [40]

One study of these filtering methods by Chowdhary [40] determined that both methods produced similar results when applied to flight parameter estimation using flight test data from three different aircraft. Parameters identified by the UKFs were found to converge fastest but also required the most processing power. No great difference between the parameters estimated using either of the Kalman filters was observed, and those parameters also matched parameters produced using the output error method. 


\subsubsection{Neural Networks}

Neural networks (NNs) belong to the class of evolutionary computing algorithms. They are a mathematical metaphor for a biological brain, mimicking the natural cluster of small units with simple functions which are interconnected to process large amounts of complex data. There are many different types of neural networks, but this section will discuss the type used most frequently in modelling and simulation, the feed-forward network with back-propagation learning [41].

An NN is made up of neurons, or nodes, which exist in a hidden layer, or "black box". This black box accepts inputs and produces outputs and is capable of being trained and of learning from specified input-output pairs. Each node contained in the black box is actually a multiple-input multiple-output mathematical function, called an activation function. The number of nodes depends on the complexity of the problem [42]. There are a variety of activation function types such as scaled sigmoid, Gaussian, sine, and hyperbolic tangent [43]. The nodes are connected and the output of each node is fed forward to at least one other node; the arrangement of these connections is called the network topology. As each output value is passed on, it is assigned a weighting function. The weighting functions can be positive or negative values, called excitatory or inhibitory, respectively. As a node receives input from other nodes, the weighted values are summed accordingly. Both the weighting functions and the topology can be randomly assigned during the creation of the $\mathrm{NN}$, though the connections only feed forward to the next layer of the network and do not create loops [43].

The NNs learn and evolve through a process called back-propagation. The network is given input-output pair examples and the back-propagation algorithm works to reduce the error of the program along the gradient of layers. It is possible to "overfit" the NNs if too many pair examples are given, as the $\mathrm{NN}$ will begin to converge on very specific examples and become intolerant of new input patterns or pairs [43]. Learning processes 
in the past did not allow for NNs to efficiently train for nonlinear problems, but the introduction of back propagation in 1986 by Rumelhart and McClelland made nonlinear NNs workable [41].

Neural networks have a broad range of uses but have been applied specifically to parameter estimation problems like aerodynamic coefficient prediction for wind tunnel data [44] and flight vehicle parameter estimations in which the aerodynamic coefficients of a flight vehicle were calculated given the initial vehicle orientation, velocities, accelerations, and control inputs [42].

One of the most unique aspects of NNs is that they do not require actual mathematical modelling of a system in order to predict the response of that system, making them ideal for predicting the behaviour of complex systems. In some cases where the design of a flight simulator includes the development of the flight model engine (rather than purchasing COTS software) NNs are used as a method of predicting aircraft behaviour [45].

\subsubsection{Genetic Algorithms}

Genetic algorithms (GAs), like NNs, belong to the class of evolutionary computing algorithms that attempt to mimic natural processes to predict behaviour and solve optimization problems ranging far beyond flight modelling. The widely adaptable nature of these algorithms allow them to solve problems ranging in fields from economics to medicine. In industry, GAs are used in production planning, scheduling, sequencing, and line balancing. In finance, GAs are used for risk assessment, modelling trading behaviour, portfolio optimization, and even stock market prediction [41].

GAs solve these problems using curve fitting, a process by which a closed form function is approximated in order to provide the line of best fit to a given data set. There are many ways to approximate a function; however, given a complex function with a nonlinear form or without information regarding derivatives, there are few available methods that work 
well. Genetic algorithms (GAs) are robust parallel heuristic search methods that often use least squares curve fitting methods to solve complex problems. With even the weakest correlations between proposed solutions and function values, a GA will almost always be able to produce a nearly optimal solution [46].

GAs have been used in flight modelling and simulation in a variety of ways such as tuning PD or PID controllers in flight simulator control systems [47, 48]. GAs are commonly paired with other optimization techniques such as particle swarm optimizations, or used to optimize the network topology and weighting functions of a neural network [44].

\subsection{Use of Stability Derivatives in Flight Dynamics Modelling}

As discussed in Chapter 4, flight simulator software generally uses either stability derivatives or blade element theory to drive the simulator flight model and the advantages and disadvantages of both were discussed. The required flight model parameters in each system differ greatly, with stability-derivative-based software requiring performance parameters (either aerodynamic coefficients or the stability derivatives themselves), and bladeelement-theory-based systems requiring physical parameters (geometry and engine power specifications). Had the flight dynamics model for X-Plane been readily available for implementation with an automated model tuning algorithm, the geometric parameters of the aircraft would have been tuned to produce the most accurate flight model possible. As this was not the case, a different math model was selected in order to aid in the development of an automated flight model tuning method with the understanding that in a future phase of development, the compatibility issue between the model tuning algorithm and the KatanaSim software would be resolved. This is discussed further in Section 5.5.

This section will focus on the derivation of the longitudinal rigid body equations of 


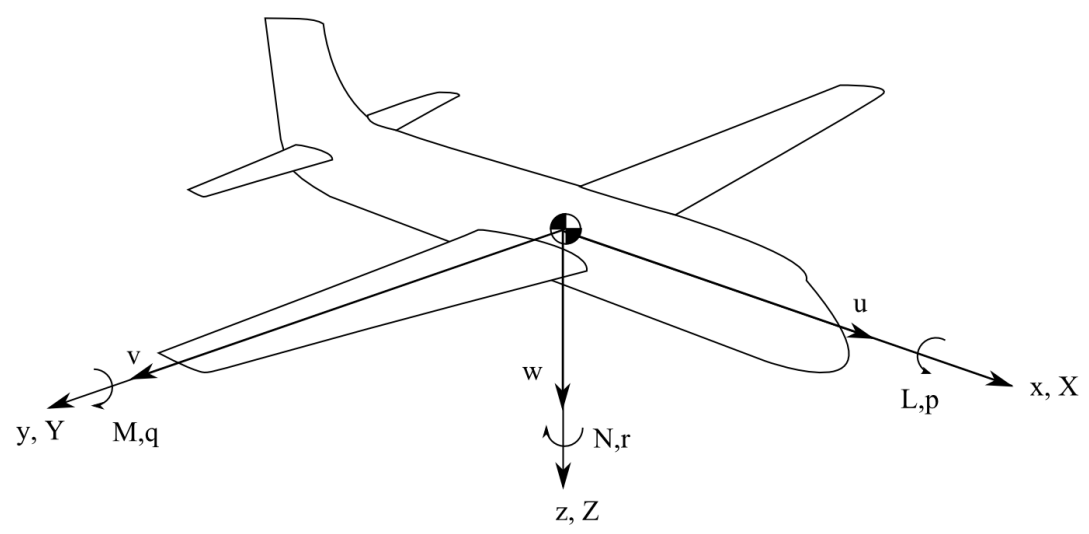

Figure 5.1: Three-dimensional definition of angular and linear velocities, forces, and axes in a body-fixed coordinate system.

motion for an aircraft for use in Section 5.4 as part of a genetic algorithm developed for model tuning. The nomenclature for the derivation is summarized in Figure 5.1 and Table 5.1.

Table 5.1: Nomenclature for 3-dimensional aircraft analysis.

\begin{tabular}{lccc}
\hline & Roll & Pitch & Yaw \\
\hline Axis & $\mathrm{x}$ & $\mathrm{y}$ & $\mathrm{Z}$ \\
Angle & $\Phi$ & $\theta$ & $\varphi$ \\
Linear velocity & $\mathrm{u}$ & $\mathrm{v}$ & $\mathrm{w}$ \\
Angular velocity & $\mathrm{p}$ & $\mathrm{q}$ & $\mathrm{r}$ \\
Aerodynamic Force & $\mathrm{X}$ & $\mathrm{Y}$ & $\mathrm{Z}$ \\
Aerodynamic Moment & $\mathrm{L}$ & $\mathrm{M}$ & $\mathrm{N}$ \\
\hline
\end{tabular}

\subsubsection{Derivation of Longitudinal Rigid Body Equations of Mo- tion}

In this section, the longitudinal equations of motion are derived, encompassing the forward and vertical motion as well as the pitch changes of the aircraft. A two-dimensional analysis was chosen as the resulting equations would be adequate for initial testing of a genetic 
algorithm before implementing the full set of equations required to define the system in three dimensions.

Starting with Newton's second law, the summations of moments and forces on the aircraft body result in

$$
\begin{aligned}
\Sigma \mathbf{F} & =m \frac{\mathrm{d} \mathbf{V}}{\mathrm{dt}} \\
\Sigma \mathbf{M} & =\frac{\mathrm{d} \mathbf{H}}{\mathrm{dt}}
\end{aligned}
$$

where $\mathrm{V}$ is the total velocity of the aircraft in an inertial reference frame and $\mathrm{H}$ is the total angular momentum in an inertial frame. For convenience, it is customary to derive vehicle equations of motion in a body-fixed local $\vec{x} \cdot \vec{y} \cdot \vec{z}$ coordinate system. Since this is a non-inertial coordinate system, the general expression for differentiation of an arbitrary vector $\vec{A}$ must be used such that

$$
\frac{\mathrm{d} \mathbf{A}}{\mathrm{dt}} \text { textinertial }_{\mathrm{dt}}=\frac{\mathrm{d} \mathbf{A}}{\text { body }}+\omega \times \mathbf{A}
$$

where $\vec{w}$ is the angular velocity of the $\vec{x} \cdot \vec{y} \cdot \vec{z}$ frame. The results in the $\mathrm{x}$ and $\mathrm{z}$ translational equations of motion being

$$
\begin{aligned}
& F_{x}=m(\dot{u}+q w) \\
& F_{z}=m(\dot{w}-q u)
\end{aligned}
$$

The vertical and horizontal components of the aircraft velocity were then defined as follows, 
where $\theta$ is the pitch angle of the aircraft:

$$
\begin{aligned}
& \frac{\mathrm{d} x}{\mathrm{dt}}=u \cos \theta+w \sin \theta \\
& \frac{\mathrm{d} z}{\mathrm{dt}}=-u \sin \theta+w \cos \theta
\end{aligned}
$$

and the gravitation forces acting along the body axes were defined as

$$
\begin{aligned}
& F_{x, g}=-m g \sin \theta \\
& F_{z, g}=m g \cos \theta
\end{aligned}
$$

From the above definitions, the rigid body equations governing aircraft motion in response to the $X$ and $Z$ aerodynamic forces, and the aerodynamic moment $M$, are written as:

$$
\begin{aligned}
X-m g \sin \theta & =m(\dot{u}+q w) \\
Z+m g \cos \theta & =m(\dot{w}-q u) \\
M & =I_{y} \dot{q}
\end{aligned}
$$

The equations above are nonlinear, coupled, and lend no direct insight as to the handling characteristics of the aircraft [49]. The linearized approximations of these equations are much more useful in analysing the stability and control characteristics of the aircraft. These approximations can be found using the small-disturbance theory in which the mo- 
tion of the aircraft is described as small perturbations about a reference condition [50]. Because of these approximations, the resulting equations of motion cannot be used to produce accurate results for manoeuvres such as spins or stalls where large-amplitude perturbations occur.

To apply the small-disturbance theory, a reference condition of straight and level unaccelerated flight was chosen and a small perturbation was considered applied to each variable found in the equations of motion, such that

$$
\begin{aligned}
u & =u_{0}+\Delta u \\
w & =w_{0}+\Delta w \\
q & =q_{0}+\Delta q \\
X & =X_{0}+\Delta X \\
Z & =Z_{0}+\Delta Z \\
\theta & =\theta_{0}-\Delta \theta
\end{aligned}
$$

Adding the perturbations to Equations 5.10, 5.11, and 5.12, the equations become

$$
\begin{aligned}
X_{0}+\Delta X-m g \sin \left(\theta_{0}+\Delta \theta\right) & =m\left[\frac{\mathrm{d}}{\mathrm{dt}}\left(u_{0}+\Delta u\right)+\left(q_{0}+\Delta q\right)\left(w_{0}+\Delta w\right)\right] \\
Z_{0}+\Delta Z+m g \cos \left(\theta_{0}+\Delta \theta\right) & =m\left[\frac{\mathrm{d}}{\mathrm{dt}}\left(w_{0}+\Delta w\right)-\left(q_{0}+\Delta q\right)\left(u_{0}+\Delta u\right)\right] \\
M_{0}+\Delta M & =I_{y}\left[\frac{\mathrm{d}}{\mathrm{dt}}\left(q_{0}+\Delta q\right)\right]
\end{aligned}
$$

Since all perturbations are assumed to be small, these equations simplify by neglecting the 
second-order products of the disturbances. It can also be noted that since the reference flight condition is assumed to be symmetric and aligned with the $x$ axis, then $q_{0}=0$ and $w_{0}=0$. Also, as the reference state was defined as being unaccelerated, then $\frac{\mathrm{d}}{\mathrm{dt}} u=0$, resulting in

$$
\begin{aligned}
X_{0}+\Delta X-m g \sin \left(\theta_{0}+\Delta \theta\right) & =m \frac{\mathrm{d}}{\mathrm{dt}} \Delta u \\
Z_{0}+\Delta Z+m g \cos \left(\theta_{0}+\Delta \theta\right) & =m\left(\frac{\mathrm{d}}{\mathrm{dt}} \Delta w-\Delta q u_{0}\right) \\
M_{0}+\Delta M & =I_{y}\left(\frac{\mathrm{d}}{\mathrm{dt}} \Delta q\right)
\end{aligned}
$$

The equations were reduced further by recognizing the reference flight conditions as

$$
\begin{array}{r}
X_{0}-m g \sin \theta_{0}=0 \\
Z_{0}+m g \cos \theta_{0}=0 \\
M_{0}=0
\end{array}
$$

Also, by approximating $\sin \Delta \theta$ as $\Delta \theta$ and $\cos \Delta \theta$ as 1 , the following trigonometric identities were applied

$$
\begin{aligned}
& \sin \left(\theta_{0}+\Delta \theta\right)=\sin \theta_{0} \cos \Delta \theta+\cos \theta_{0} \sin \Delta \theta \approx \sin \theta_{0}+\Delta \theta \cos \theta_{0} \\
& \cos \left(\theta_{0}+\Delta \theta\right)=\cos \theta_{0} \cos \Delta \theta-\sin \theta_{0} \sin \Delta \theta \approx \cos \theta_{0}-\Delta \theta \sin \theta_{0}
\end{aligned}
$$


The reduced equation set can be written as

$$
\begin{aligned}
\Delta X-m g \Delta \theta \cos \theta_{0} & =m \frac{\mathrm{d}}{\mathrm{dt}} \Delta u \\
\Delta Z-m g \Delta \theta \sin \theta_{0} & =m\left(\frac{\mathrm{d}}{\mathrm{dt}} \Delta w-\Delta q u_{0}\right) \\
\Delta M & =I_{y} \frac{\mathrm{d}}{\mathrm{dt}} \Delta q
\end{aligned}
$$

In the above equations, $\Delta X, \Delta Y$, and $\Delta M$ represent the change in aerodynamic and propulsive forces and moments, and are functions of the motion variables. The stability derivatives considered to have a non-negligible effect on the aircraft performance, as defined by Nelson [50], are given by

$$
\begin{aligned}
\Delta X & =\frac{\partial X}{\partial u} \Delta u+\frac{\partial X}{\partial w} \Delta w+\frac{\partial X}{\partial \delta_{e}} \Delta \delta_{e}+\frac{\partial X}{\partial \delta_{T}} \Delta \delta_{T} \\
\Delta Z & =\frac{\partial Z}{\partial u} \Delta u+\frac{\partial Z}{\partial w} \Delta w+\frac{\partial Z}{\partial \dot{w}} \Delta \dot{w}+\frac{\partial Z}{\partial q} \Delta q+\frac{\partial Z}{\partial \delta_{e}} \Delta \delta_{e}+\frac{\partial Z}{\partial \delta_{T}} \Delta \delta_{T} \\
\Delta M & =\frac{\partial M}{\partial u} \Delta u+\frac{\partial M}{\partial w} \Delta w+\frac{\partial M}{\partial \dot{w}} \Delta \dot{w}+\frac{\partial M}{\partial q} \Delta q+\frac{\partial M}{\partial \delta_{e}} \Delta \delta_{e}+\frac{\partial M}{\partial \delta_{T}} \Delta \delta_{T}
\end{aligned}
$$

where $\delta_{T}$ and $\delta_{e}$ are the change in thrust and change in elevator input, respectively. Substituting these expressions into the force and moment equations yields: 


$$
\begin{aligned}
m \frac{\mathrm{d}}{\mathrm{dt}} \Delta u= & \frac{\partial X}{\partial u} \Delta u+\frac{\partial X}{\partial w} \Delta w+\frac{\partial X}{\partial \delta_{e}} \Delta \delta_{e}+\frac{\partial X}{\partial \delta_{T}} \Delta \delta_{T} \\
& -m g \Delta \theta \cos \theta_{0} \\
m\left(\frac{\mathrm{d}}{\mathrm{dt}} \Delta w-\Delta q u_{0}\right)= & \frac{\partial Z}{\partial u} \Delta u+\frac{\partial Z}{\partial w} \Delta w+\frac{\partial Z}{\partial \dot{w}} \Delta \dot{w}+\frac{\partial Z}{\partial q} \Delta q+\frac{\partial Z}{\partial \delta_{e}} \Delta \delta_{e} \\
& +\frac{\partial Z}{\partial \delta_{T}} \Delta \delta_{T}-m g \Delta \theta \sin \theta_{0} \\
I_{y} \frac{\mathrm{d}}{\mathrm{dt}} \Delta q= & \frac{\partial M}{\partial u} \Delta u+\frac{\partial M}{\partial w} \Delta w+\frac{\partial M}{\partial \dot{w}} \Delta \dot{w}+\frac{\partial M}{\partial q} \Delta q+\frac{\partial M}{\partial \delta_{e}} \Delta \delta_{e} \\
& +\frac{\partial M}{\partial \delta_{T}} \Delta \delta_{T}
\end{aligned}
$$

The force equations are then divided through by mass, at which point a new notation is implemented for the stability derivatives.

$$
X_{u}=\frac{\partial X / \partial u}{m}, Z_{u}=\frac{\partial Z / \partial u}{m}, M_{u}=\frac{\partial X / \partial u}{I_{y}}, \text { etc... }
$$

for all 14 longitudinal stability derivatives. Rearranging the equations to group like terms results in the final form of the linearized small-disturbance longitudinal rigid body equations of motion: 


$$
\begin{aligned}
X_{\delta_{e}} \Delta \delta_{e}+X_{\delta_{T}} \Delta \delta_{T}= & \left(\frac{\mathrm{d}}{\mathrm{dt}}-X_{u}\right) \Delta u-X_{w} \Delta w+g \cos \theta_{0} \Delta \theta \\
Z_{\delta_{e}} \Delta \delta_{e}+Z_{\delta_{T}} \Delta \delta_{T}= & -Z_{u} \Delta u+\left[\left(1-Z_{\dot{w}}\right) \frac{\mathrm{d}}{\mathrm{dt}}-Z_{w}\right] \Delta w \\
& -\left[\left(u_{0}+Z_{q}\right) \frac{\mathrm{d}}{\mathrm{dt}}-g \sin \theta_{0}\right] \Delta \theta \\
M_{\delta_{e}} \Delta \delta_{e}+M_{\delta_{T}} \Delta \delta_{T}= & -M_{u} \Delta u-\left(M_{\dot{w}} \frac{\mathrm{d}}{\mathrm{dt}}+M_{w}\right) \Delta w+\left(\frac{\mathrm{d}^{2}}{\mathrm{dt}^{2}}-M_{q} \frac{\mathrm{d}}{\mathrm{dt}}\right) \Delta \theta(5
\end{aligned}
$$

To simplify the use of these equations, they were rewritten in state-space form as a set of first-order differential equations of the form

$$
I_{\eta} \dot{x}=A x+B \eta
$$

The longitudinal state variable vector and control vector were, respectively, defined as

$$
\begin{aligned}
& \mathbf{x}=\left[\begin{array}{lll}
u & w & q \\
\theta
\end{array}\right]^{T} \\
& \boldsymbol{\eta}=\left[\begin{array}{ll}
\delta_{e} & \delta_{T}
\end{array}\right]^{T}
\end{aligned}
$$

Matrices $I_{\eta}, A$, and $B_{\eta}$ were defined as written below, although $Z_{\dot{w}}$ and $Z_{q}$ were neglected in the final form as they are known to contribute very little to the aircraft response [49] 


$$
\begin{aligned}
& \boldsymbol{I}_{\boldsymbol{\eta}}=\left[\begin{array}{cccc}
1 & 0 & 0 & 0 \\
0 & 1-Z_{\dot{w}} & 0 & 0 \\
0 & -M_{\dot{w}} & 1 & 0 \\
0 & 0 & 0 & 0
\end{array}\right] \\
& \boldsymbol{A}=\left[\begin{array}{cccc}
X_{u} & X_{w} & 0 & -g \cos \theta_{0} \\
Z_{u} & Z_{w} & u_{0} & -g \sin \theta_{0} \\
M_{u}+M_{\dot{w}} & M_{w}+M_{\dot{w}} Z_{w} & M_{q}+M_{\dot{w}} u_{0} & -M_{\dot{w}} g \sin \theta_{0} \\
0 & 0 & 1 & 0
\end{array}\right] \\
& \boldsymbol{B}_{\boldsymbol{\eta}}=\left[\begin{array}{cc}
X_{\delta_{e}} & X_{\delta_{T}} \\
Z_{\delta_{e}} & Z_{\delta_{T}} \\
M_{\delta_{e}}+M_{\dot{w}} Z_{\delta_{e}} & M_{\delta_{T}}+M_{\dot{w}} Z_{\delta_{T}} \\
0 & 0
\end{array}\right]
\end{aligned}
$$

The final state-space form appears as follows 


$$
\begin{aligned}
\left\{\begin{array}{c}
\dot{u} \\
\dot{w} \\
\dot{q} \\
\dot{\theta}
\end{array}\right\}= & {\left[\begin{array}{cccc}
X_{u} & X_{w} & 0 & -g \cos \theta_{0} \\
Z_{u} & Z_{w} & u_{0} & -g \sin \theta_{0} \\
M_{u}+M_{\dot{w}} & M_{w}+M_{\dot{w}} Z_{w} & M_{q}+M_{\dot{w}} u_{0} & -M_{\dot{w}} g \sin \theta_{0} \\
0 & 0 & 1 & 0
\end{array}\right]\left\{\begin{array}{l}
u \\
w \\
q \\
+
\end{array}\right.} \\
& +\left[\begin{array}{ccc}
X_{\delta_{e}} & X_{\delta_{T}} \\
Z_{\delta_{e}} & & Z_{\delta_{T}} \\
M_{\delta_{e}}+M_{\dot{w}} Z_{\delta_{e}} & M_{\delta_{T}}+M_{\dot{w}} Z_{\delta_{T}} \\
0 & & 0
\end{array}\right]\left\{\begin{array}{l}
\delta_{e} \\
\delta_{T}
\end{array}\right\}
\end{aligned}
$$

These equations are used, along with the lateral-directional equations of motion, in commercially-available flight simulator software to define the performance of an aircraft. When flight test data is available, these equations can be readily used with a genetic algorithm to tune the stability derivatives of an aircraft. This idea will be expanded upon and demonstrated in Section 5.4.

\subsection{Development of a Genetic Algorithm for Auto- mated Flight Model Tuning}

In this section, an explanation of how the theory of evolution is applied to GAs and the subsequent development of a GA for parameter estimation in a variety of dynamic models is presented. 


\subsubsection{Modelling Evolution}

The genotype of an organism is the information stored in the genetic code of that organism. The genotype holds the blueprints for that organism's appearance and behaviour, which in turn is called its phenotype [41]. Given an initial population and an environmental pressure which favours a particular phenotype, the genetic code of the population will trend towards those genotypes which can produce the most suitable phenotypes for the environment. New genotypes are produced with each generation due to the sharing of chromosomes through reproduction and the introduction of new chromosomes through random mutations. As the weaker genotypes, less suited to their environment, do not reproduce, beneficial chromosomes and mutations are passed on to future generations through a process known as natural selection [51].

The principle of natural selection of genotypes and phenotypes can be mimicked in modelling. The coefficients in an equation or set of equations are assembled into a bit string, analogous to the genotype of an organism. The behaviour of the system with those coefficients is the phenotype. A population is created with a number of different genotypes. An environmental pressure is then applied by designating the desired behaviour of the system and culling those genotypes which do not closely adhere to that behaviour.

The population, or solution set, can be as small or as large as desired, with some considerations. A large population size will require more computation power and/or time for evaluation, while a small population would likely take longer to evolve and find the correct solution set. While one of the greatest strengths of a GA is the ability it has to avoid convergence on local optima by completing a thorough search of the possible solution space, it is also a weakness in that the time it can take to converge on a solution may be increased. 


\section{Survival of the Fittest}

The first generation of genotypes is chosen randomly; the solution set is filled with coefficients assigned by a random number generator but constrained to feasible parameter ranges. The phenotype of each member of the population is evaluated and compared to the desired phenotype. A fitness function such as the sum of squares error (SSE) is used to compare the two phenotypes, and the population member is assigned a fitness value. This fitness value determines whether or not that particular solution will be culled from the population, or whether it will be part of the next generation. The solutions are ranked from highest to lowest fitness, and the highest $50 \%$ are marked as the survivors and carried over to the next generation. These population members will be used as the "parents" to create offspring.

\section{Children}

After the least fit population members are culled, it is desirable to repopulate and keep the population size constant from one generation to the next. A portion of the population will be filled by "breeding" children from the remaining parents. The child genotypes can be created in two ways, by crossover of genetic material from both parents, or by averaging the genetic information [41]. In a crossover, certain bits from the first parent's genotype are exchanged with the second parent's genotype. In a 1-point crossover, a single random point is selected, the parents are split at this point (denoted by a colon in the example below), and the sections are exchanged between parents. For example, if two parent genotypes were

$$
\begin{aligned}
& X=1010: 10 \\
& Y=2020: 20
\end{aligned}
$$


with each number representing a chromosome in the genotype, then one potential 1-point crossover would result in children

$$
\begin{aligned}
& Z 1=1010: 20 \\
& Z 2=2020: 10
\end{aligned}
$$

In the bit strings representing the genotype of the parents, up to half of the chromosomes in the bit string can be exchanged during breeding; the number of chromosome crossover points can be increased or decreased in order to speed or slow the rate at which the space of all possible solutions is explored.

The second type of breeding which can occur is an arithmetic crossover which creates children "between" the parents [52]. For example, if two parent genotypes were

$$
\begin{aligned}
& X=10,10,10 \\
& Y=20,20,20
\end{aligned}
$$

then the child's chromosomes would be calculated as

$$
\begin{gathered}
Z_{i}=\alpha X_{i}+(1-\alpha) Y_{i}, \\
\text { where } \alpha: 0 \leq \alpha \leq 1
\end{gathered}
$$

and one potential resulting child, using $\alpha=0.5$, would be

$$
Y_{1}=15,15,15
$$

Any number of chromosomes can be selected for modification using this method, while $\alpha$ can be kept constant, vary from chromosome to chromosome, or be chosen at random for each use. There are infinite possibilities for breeding children by varying parameter $\alpha$, the number of chromosomes affected in each combination, how many children are produced from each parent set, and the selection of parents for breeding. 


\section{Mutations}

After the children have been bred, the remainder of the population can be filled with mutations. Mutations are a way of increasing the number of paths being explored in the solution space and decreasing the likelihood of convergence on a local optimum [41].

Mutations are created by selecting one or more chromosomes in a given genotype (parent or child) to be replaced by a randomly generated value. A higher frequency of chromosome mutation will result in greater exploration of the possible solution space, but too high a frequency could slow solution convergence.

\section{Creation of the Next Generation and Solution Convergence}

After the first generation of randomly created genotypes has been evaluated for fitness and then culled, the population is repopulated with children and mutations. This new generation is then evaluated for fitness and once again, $50 \%$ of the population is culled to eliminate the genotypes with the least desirable phenotypes. The process of breeding, mutating, and culling is repeated, with more advantageous genetic traits being discovered and preserved in each generation, until convergence on an acceptable solution is achieved.

A GA can be set to terminate once the desired behaviour of a genotype has been achieved and the fitness function is within specified limits. Termination can also be initiated by an improvement metric that flags the end of the algorithm once new generations have stopped showing improvements in behaviour.

\section{Sequential Evolution Mechanisms}

One particular consideration for some curve-fit-optimization problems is how the contribution of each element to the overall fitness of a genotype can vary significantly, e.g., the higher-order coefficients in a polynomial will have a greater effect on the phenotype than the lower-order coefficients. If breeding and mutations affect each chromosome equally, the 
evolution of the genotype will focus on the more influential chromosomes at the expense of the less influential chromosomes.

One solution for this phenomenon is the implementation of sequential evolution mechanisms (SEMs) as developed by Gulsen, Smith, and Tate [46]. In this procedure, chromosomes are grouped by the magnitude of their effect on the phenotype, and only one group at a time will be activated during the breeding and mutation sequences used to create the next generation. In the most extreme form of SEM, each chromosome could evolve separately.

In most SEMs, each chromosome group is activated sequentially so that each group is given an equal number of evolutions before convergence. In complex problems where certain chromosome groups are particularly slow to evolve, it is possible to assign multiple evolutionary iterations [46].

\subsubsection{Development of the Genetic Algorithm}

In order to develop a curve fitting GA which could be used for flight model tuning, small dynamics problems were first tackled in order to refine the selection, breeding, and mutation mechanisms. These problems were first prototyped using MATLAB, and then in Fortran as it greatly reduced the required computation time.

\section{Development Example 1: Cart with Spring and Damper}

The first problem was based on a basic cart, spring, and damper system as shown in Figure 5.2 and was programmed in MATLAB. A reference data set was created by assigning values for the mass of the cart, the spring constant, and the damping constant. The dynamics of the cart were then calculated, given an initial force, cart position, and cart velocity. The position of the cart was recorded at intervals of 0.05 seconds for a total of 30 seconds. This array became the reference data against which future possible solution 


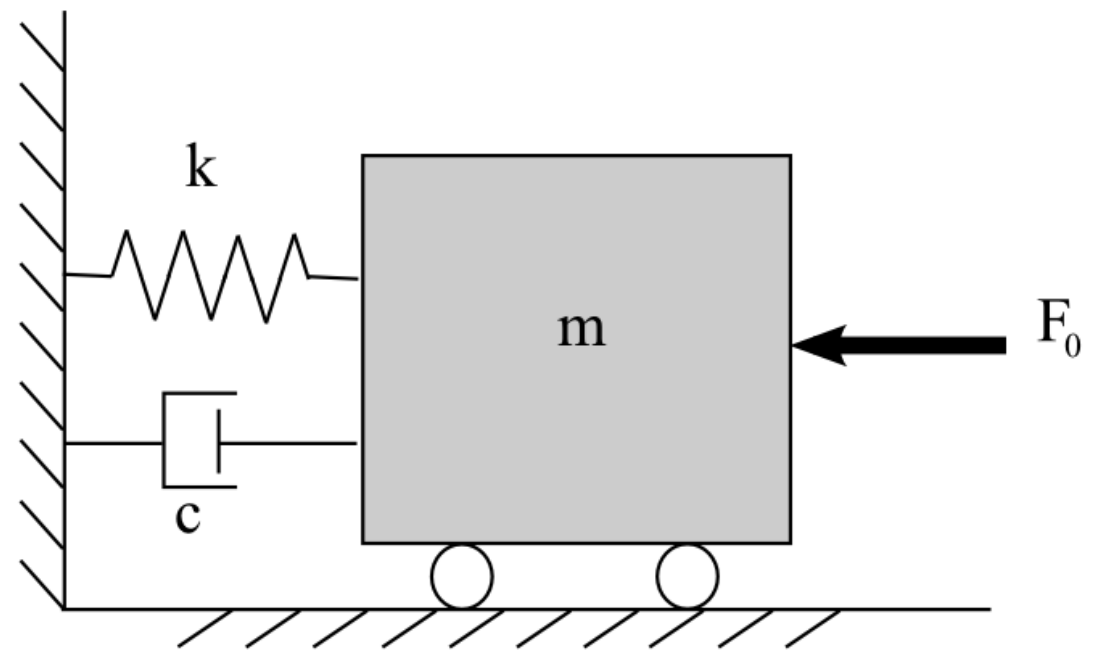

Figure 5.2: System consisting of a cart with spring and damper with an initial applied force, used for the creation of a simple curve-fitting GA.

sets would be compared.

The first GA was designed to determine the mass of the cart $m$, linear spring constant $k$, and viscous damping constant $c$, given the initial conditions of the system and the behaviour of the cart. This example problem was used to extensively test the performance of the genetic algorithm with a variety of population sizes and child and mutation creation methods.

Due to the simplicity of the problem, an initial population size of forty was chosen. The initial twenty coefficient sets, which would act as the first possible parent genotypes, were randomly generated given certain assumptions on the upper limits of the possible values of $m, c$, and $k$. The response of the system was calculated for each combination of $m, c$, and $k$ and then compared to the reference behaviour array. The SSE was calculated for each. These twenty genotypes and their errors were stored in a matrix $Q$ with the form

$$
\mathbf{Q}=[\mathrm{m} \mathrm{c} \mathrm{k} \mathrm{SSE}] \text {. }
$$

$Q$ was then sorted from smallest to largest SSE, and the ten genotypes with the smallest SSE were designated as parents. Parents 1 and 2 were paired together to create two 
children, Parents 3 and 4 were paired together to create two children, and so on, so that the ten designated parent genotypes produced ten new genotypes. The $i^{\text {th }}$ chromosomes of the children were found with

$$
\begin{aligned}
& {\text { Child } 1_{i}}=\alpha * \text { Parent }_{i}+(1-\alpha) * \text { Parent } 2_{i} \\
& \text { Child } 2_{i}=\alpha * \text { Parent } 2_{i}+(1-\alpha) * \text { Parent } 1_{i}
\end{aligned}
$$

with the best tuning performance of the algorithm occurring when $\alpha$ was equal to 0.35 .

The mutation driver in this GA was applied to the children. One coefficient from each solution was chosen for mutation, and a value $Z$ was added to it. The value $Z$ was defined as a random number between -2 and 2 multiplied by the range of the values of that chromosome in the population. This mutation method was taken from Gulsen [46]. It was discovered in the course of testing that as the algorithm approached convergence, the range of the chromosome values also diminished as the optimized parameter took on the behaviour of a dominant chromosome and propagated quickly through the population. This had the effect of lessening the effect of mutations on the values and occasionally leading to convergence at a local, rather than global, minima. To avoid this effect, a second mutation method was established. Once convergence of the SSE had reached a certain threshold, the mutation value $Z$ would be defined as a random number between -2 and 2 multiplied by $10 \%$ of the value of that chromosome.

Once the ten child genotypes and the ten mutation genotypes had been created, they were added to the original twenty randomly generated genotypes, to complete the population set. The behaviour of the genotypes (the dynamics of the cart system) were then evaluated and the entire population was reordered from smallest to largest SSE. The twenty genotypes with the highest SSEs were culled and the next generation of children and mutations were created. This continued until the lowest SSE was less than 0.001.

After confirmation that this GA worked well in determining the mass, spring constant, and damping constant of the system, the size of the population was increased to 1000 . 


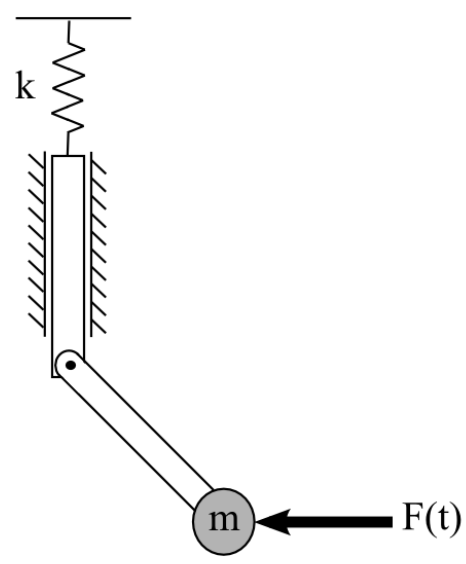

Figure 5.3: Sliding pendulum system used as a test case in the development of the curve fitting genetic algorithm.

This increase in size greatly decreased the number of algorithm iterations, or generations, required to reach convergence.

The final version of the genetic algorithm was used to solve for the unknown system coefficients 550 times to establish the repeatability of the genetic algorithm. The average error between an actual coefficient and a coefficient tuned by the algorithm was $0.33 \%$ and the largest error was $0.92 \%$.

\section{Development Example 2: Sliding Pendulum}

In the second phase of development, the GA was used to find the unknown mass and spring constant in a sliding pendulum system, shown in Figure 5.3, given a time-dependent forcing function. While the GA was used to only find two, rather than three unknown coefficients, this system provided an exercise in programming the slightly more complex dynamics including multiple coupled degrees of freedom and nonlinear behaviour associated with the sliding pendulum. The GA was successfully programmed both in MATLAB and Fortran, the latter of which had a much faster solution time and allowed for increased control over subroutines required in the algorithm, such as sorting functions and random number 
generators.

This phase of algorithm development was less focused on the output results of the algorithm, as the tuning ability of the algorithm had been thoroughly tested with the previous example problem. This second example problem was simply to confirm the algorithm could perform equally well when tuning systems with multiple coupled degrees of freedom, as well as switching programming languages.

\section{Development Example 3: Planes and Polynomials}

In the third phase of development, the GA was used to solve for the coefficients of a variety of polynomials describing curves and planes. The objective of this phase was to evaluate the ability of the algorithm to handle a larger number of unknown coefficients than had previously been tested. Populations of up to 5000 were tested with polynomials with as many as 15 unknown coefficients. The polynomial equations were chosen to represent a wide variety of curve shapes, sizes, and complexities. No unforeseen problems with the algorithm were encountered and model convergence was achieved for all cases.

\subsection{Tuning Stability Derivatives Using the Developed Genetic Algorithm}

As described, the GA has successfully been used to solve a variety of dynamic and mathematical problems with relative ease. As a tuning method, it did not require manual iterations, and the only information required for a system was the equations of motion and a set of reference test data for comparison. The final step in successfully applying the GA to flight model tuning was to use the linearized small-disturbance rigid body equations of motion to determine the stability derivatives of an aircraft based on recorded flight dynamics data. 
Research into industry-standard methods for model tuning appeared to show that, while research has been conducted on the use of GAs in flight parameter identification, the GAs are almost exclusively used in tandem with other parameter estimation techniques. The GAs are used to optimize other algorithms and are popular in their ability to improve neural network performance; however, the GAs are rarely if ever applied as a parameter estimation method on their own.

This investigation into the use of the GA for flight parameter identification was spurred on by the simplicity, and the proven curve-fitting ability, of GAs. GAs, unlike the output error method, are capable of avoiding local minimums in favour of the global minima. They also require less computational power than the Kalman filters. A GA was chosen over a neural network because of the presence of a known mathematical model. While both methods can be used for parameter estimation, the design and implementation of a GA would be much simpler than that of a neural network. 


\subsubsection{Tuning of Two-dimensional Stability Derivatives}

As was established in Section 5.2.1, Equation 5.49, the longitudinal equations of motion for an aircraft can be written in state-space form as

$$
\begin{aligned}
\left\{\begin{array}{c}
\dot{u} \\
\dot{w} \\
\dot{q} \\
\dot{\theta}
\end{array}\right\}= & {\left[\begin{array}{cccc}
X_{u} & X_{w} & 0 & -g \cos \theta_{0} \\
Z_{u} & Z_{w} & u_{0} & -g \sin \theta_{0} \\
M_{u}+M_{\dot{w}} & M_{w}+M_{\dot{w}} Z_{w} & M_{q}+M_{\dot{w}} u_{0} & -M_{\dot{w}} g \sin \theta_{0} \\
0 & 0 & 1 & 0
\end{array}\right]\left\{\begin{array}{l}
u \\
w \\
q \\
\theta
\end{array}\right\} } \\
& +\left[\begin{array}{ccc}
X_{\delta_{e}} & X_{\delta_{T}} \\
Z_{\delta_{e}} & & Z_{\delta_{T}} \\
M_{\delta_{e}}+M_{\dot{w}} Z_{\delta_{e}} & M_{\delta_{T}}+M_{\dot{w}} Z_{\delta_{T}} \\
0 & 0
\end{array}\right.
\end{aligned}
$$

The state variable vector and the control vector defined the required inputs to the algorithm. These inputs could be acquired either through flight testing or through X-Plane output files which log the performance data of a simulated aircraft flown in X-Plane. Due to a lack of aircraft availability at OAS Flight Centre and poor winter weather conditions, flight testing was delayed several months and performed late in the project. To avoid delays in the validation of the genetic algorithm, X-Plane output data were used instead of flight test data. A Cessna 172 flight model from Laminar Research was used as it was a fully-validated flight model built by Laminar Research, the makers of X-Plane. The XPlane output file contained the instantaneous roll and pitch angles, angular rates, control surface deflection angles, angle of attack, angle of sideslip, and thrust of the aircraft. Unfortunately, the velocities were output using an earth-fixed reference system which required the calculation of $u$ and $w$ to be added to the GA program between reading the input files and creating the reference files to be used for curve fitting. These calculations 
were performed by simply finding the total velocity of the aircraft using $V_{x}, V_{y}$, and $V_{z}$ given by X-Plane and using the given pitch angle, $\theta$, to resolve the velocity into $u$ and $v$ components. Velocity components $u, w$, and $q$ were then differentiated with respect to time to create the reference performance curves: $\dot{u}, \dot{w}$, and $\dot{q}$.

Once the inputs and reference performance curves had been defined, the tuning parameters were set as the 14 stability derivatives used in defining the longitudinal motion of the aircraft. The pseudo-random number generator used to create the initial population was set to generate numbers between -1.0 and 1.0 but no limitations were put on how high or low the mutations could push the coefficients during the evolutionary process.

The X-Plane output files used for the two-dimensional analysis came from a flight flown without any roll or yaw inputs. Only changes in pitch and power were made. The algorithm was initiated using the X-Plane output as the input file for the program. The algorithm converged with an SSE of 79.2 for the 1500 performance points being matched. Figures 5.4, 5.5, and 5.6 show the agreement between the reference curves and the curves produced using the tuned stability derivatives.

\subsubsection{Tuning of Three-dimensional Stability Derivatives}

Once tuning the longitudinal stability derivatives using the GA had been proven possible using the two-dimensional formulation, it was a natural next step to expand the algorithm to perform a three-dimensional analysis which would include the lateral and directional stability derivatives as well. The state-space form of the lateral and directional equations [50] is 


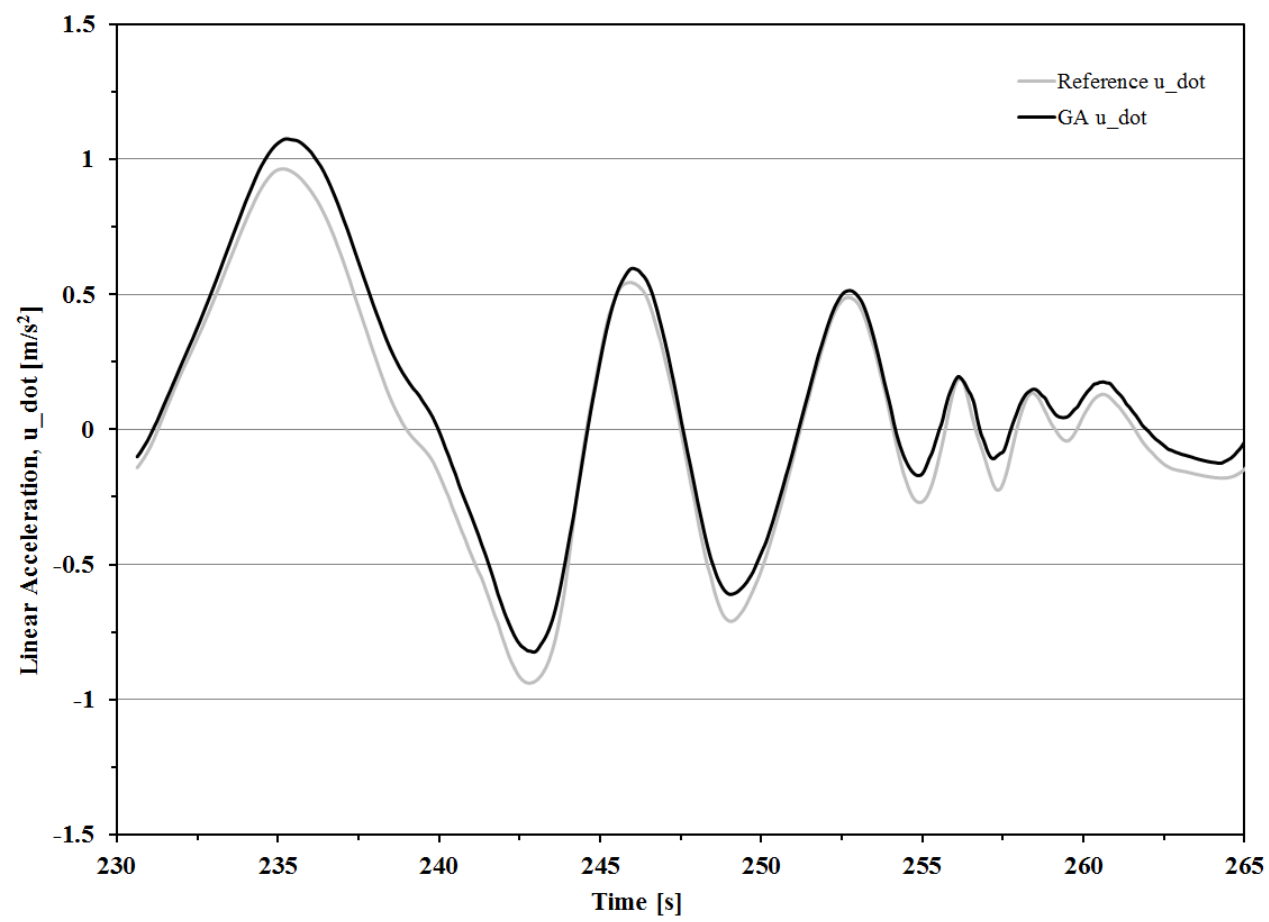

Figure 5.4: Plot of linear acceleration $\dot{u}$ versus time, for calculated data from the GA (black line) and reference data from X-Plane (gray line) in two dimensions.

$$
\left\{\begin{array}{c}
\dot{v} \\
\dot{p} \\
\dot{r} \\
\dot{\Phi}
\end{array}\right\}=\left[\begin{array}{cccc}
Y_{v} & Y_{p} & -\left(u_{0}-Y_{r}\right) & g \cos \theta_{0} \\
L_{v} & L_{p} & L_{r} & 0 \\
N_{v} & N_{p} & N_{r} & 0 \\
0 & 1 & 0 & 0
\end{array}\right]\left\{\begin{array}{l}
v \\
p \\
r \\
\Phi
\end{array}\right\}+\left[\begin{array}{cc}
0 & Y_{\delta_{r}} \\
L_{\delta_{a}} & L_{\delta_{r}} \\
N_{\delta_{a}} & N_{\delta_{r}} \\
0 & 0
\end{array}\right]\left\{\begin{array}{l}
\delta_{a} \\
\delta_{r}
\end{array}\right\}
$$

where $\delta_{a}$ and $\delta_{r}$ are the change in aileron and rudder deflection respectively. The longitudinal stability derivatives, remain the same despite the derivations of the two-dimensional and three-dimensional equations of motion differing slightly. The equations of motion in the GA were expanded to include the equations for accelerations $\dot{v}, \dot{p}$, and $\dot{r}$ and the additional 14 stability derivatives present in Equation 5.50 were added to the tuning parameters resulting in a total of 28 . 


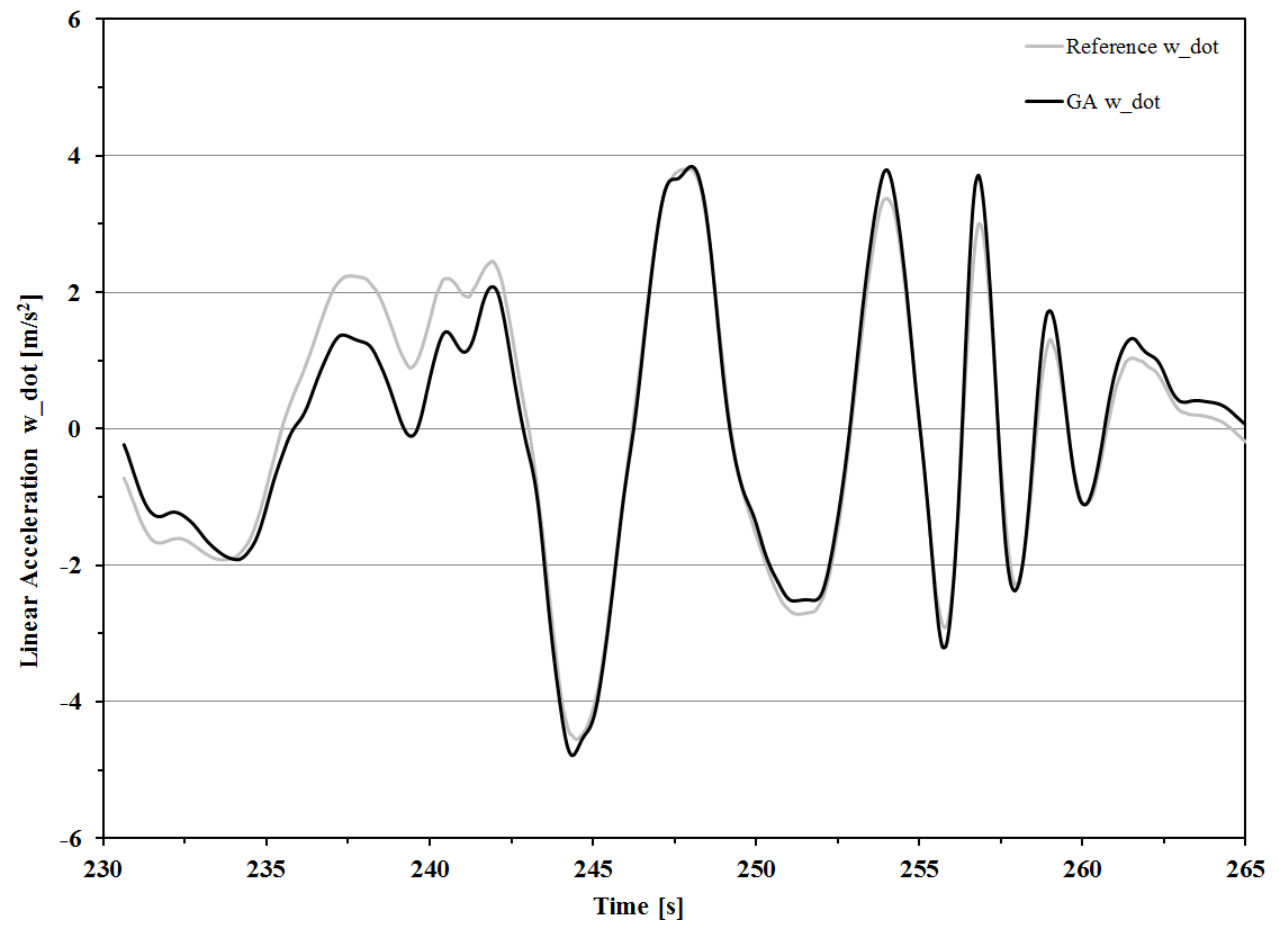

Figure 5.5: Plot of linear acceleration $\dot{w}$ versus time, for calculated data from the GA (black line) and reference data from X-Plane (gray line) in two dimensions.

As with the two-dimensional calculations, $u, v$, and $w$ had to be calculated from the velocities given by X-Plane. To do so required not just the three velocity components, $V_{x}$, $V_{y}$, and $V_{z}$, but also the sideslip angle, $\beta$, and angle of attack, $\alpha$, which were included in the output file created by X-Plane. The following three equations were used to determine $u, v$, and $w[50]$ :

$$
\begin{aligned}
V & =\sqrt{\left(V_{x}^{2}+V_{y}^{2}+V_{z}^{2}\right)}=\sqrt{u^{2}+v^{2}+w^{2}} \\
\tan \alpha & =\frac{w}{u} \\
\sin \beta & =\frac{v}{V}
\end{aligned}
$$

A new input file was created by flying a flight in X-Plane which included pitch, roll, 


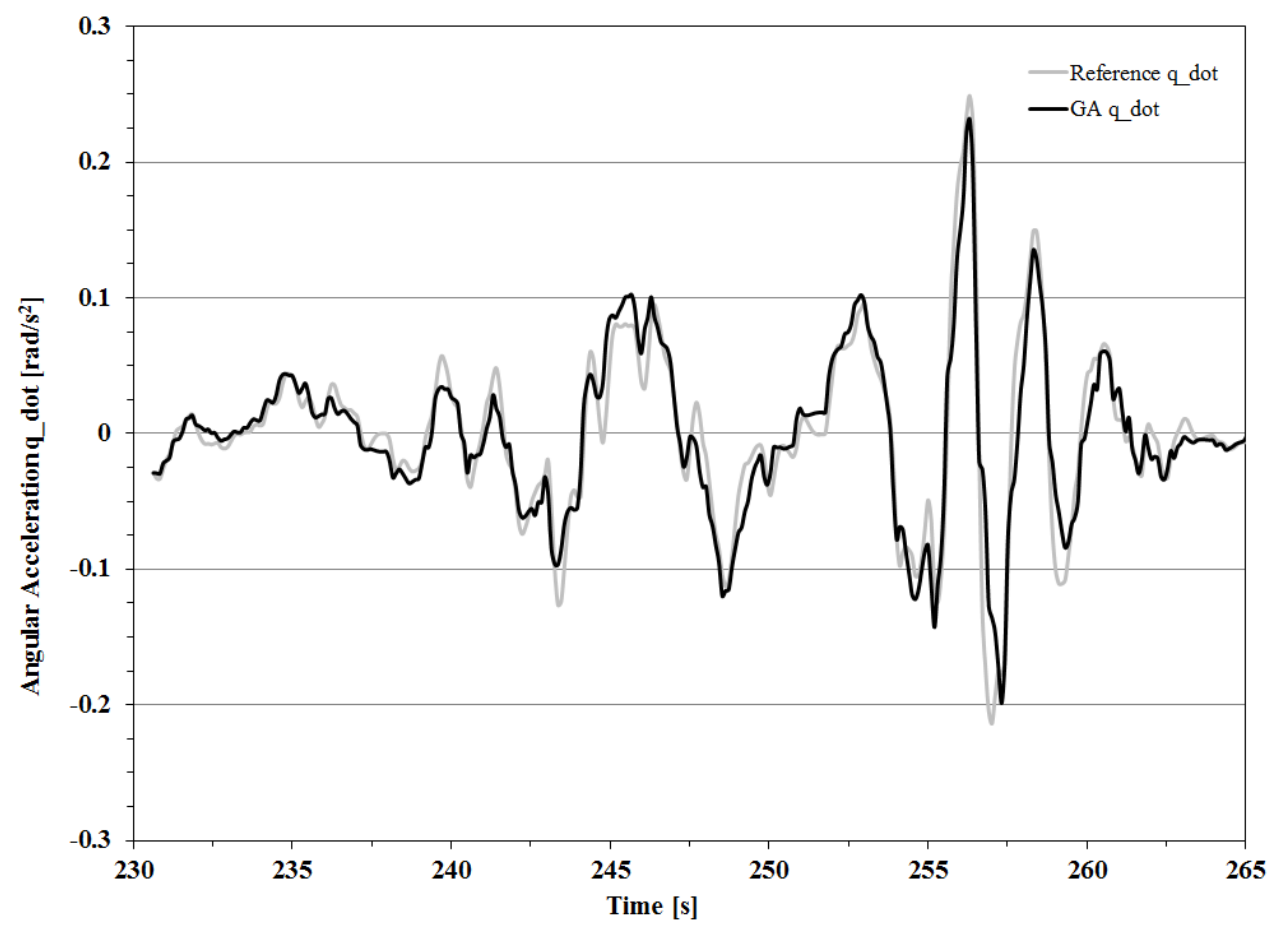

Figure 5.6: Plot of angular acceleration $\dot{q}$ versus time, for calculated data from the GA (black line) and reference data from X-Plane (gray line) in two dimensions.

yaw, and throttle inputs. The GA converged with an SSE of 74.5 for the 2280 performance points being matched. The reference accelerations and those produced by the 28 tuned stability derivatives were plotted and the results are shown in Figures 5.7 through 5.12.

\subsection{Discussion}

The genetic algorithm that was developed proved to be a very capable and adaptable parameter estimation method. The ability of the GA to tune the stability derivatives defining the equations of motion of an aircraft was tested using simulated flight test data from XPlane. The GA was able to optimize the coefficients to produce performance curves that closely match the reference curves. Some variations in the curves were seen but these were not unexpected. Because the reference curves originate from the blade-element-theorybased math model in X-Plane, while the algorithm calculates the performance curves 


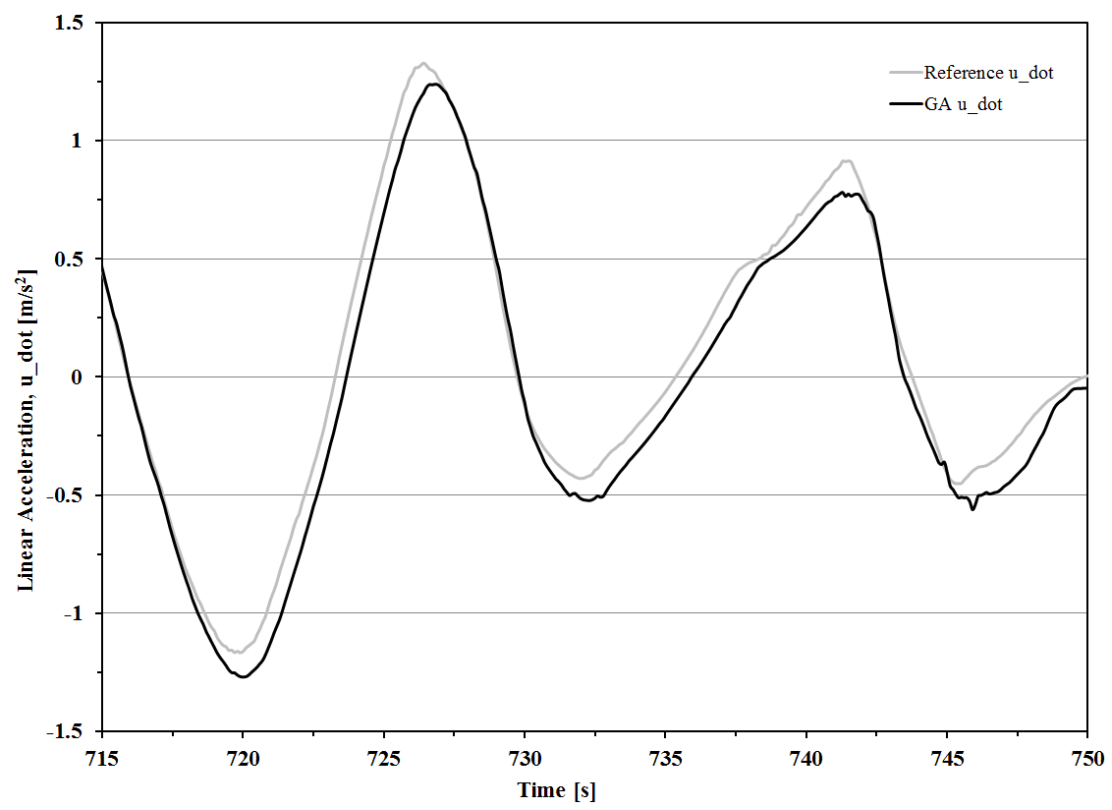

Figure 5.7: Plot of linear acceleration $\dot{u}$ versus time, for calculated data from the GA (black line) and reference data from X-Plane (gray line) in three dimensions.

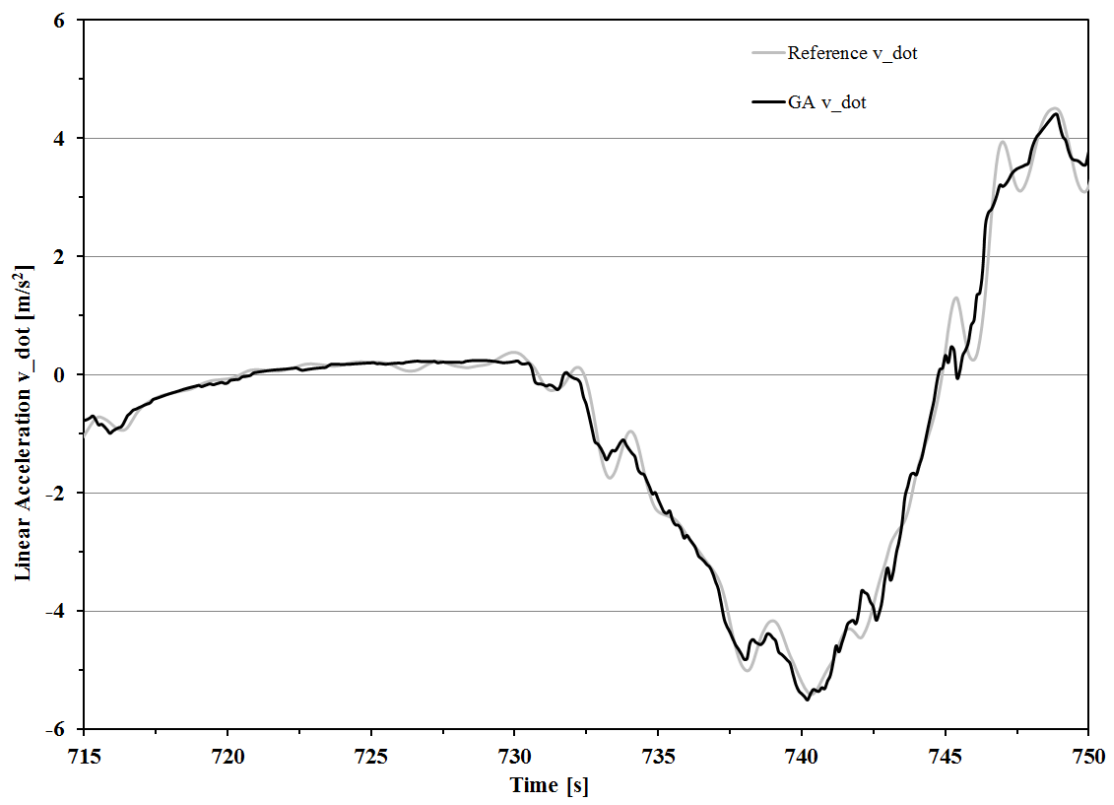

Figure 5.8: Plot of linear acceleration $\dot{v}$ versus time, for calculated data from the GA (black line) and reference data from X-Plane (gray line) in three dimensions. 


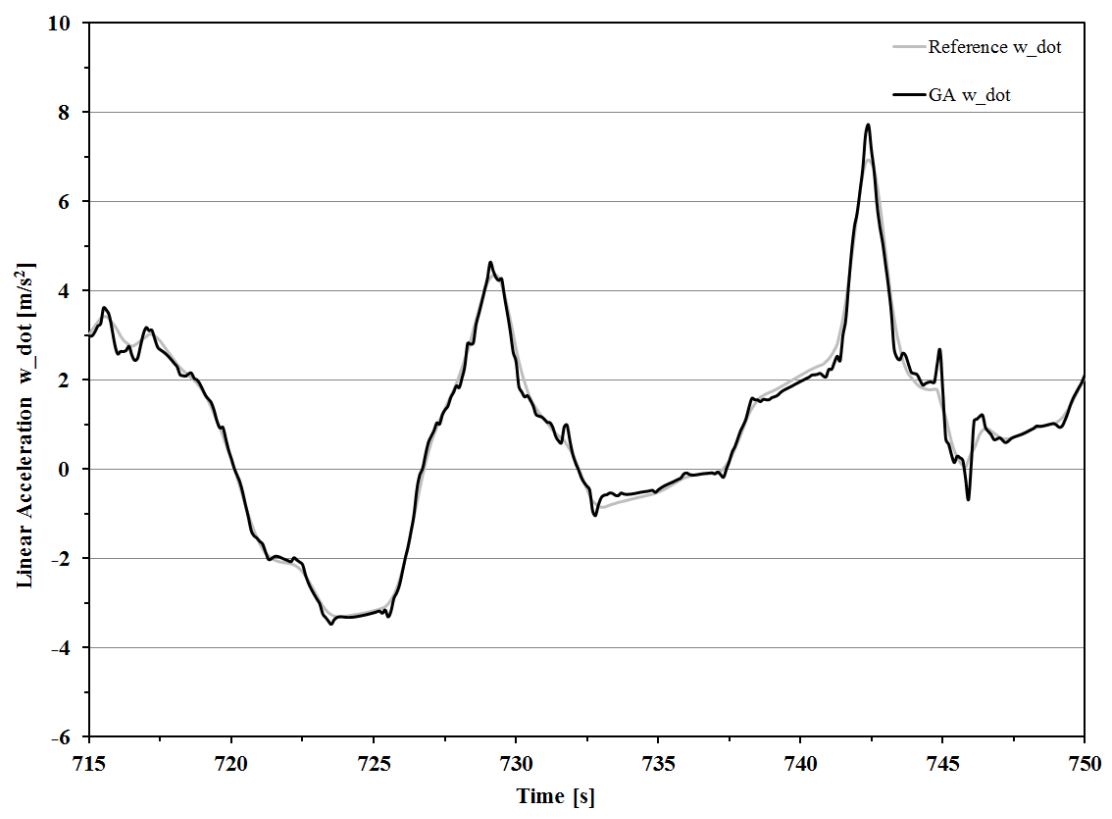

Figure 5.9: Plot of linear acceleration $\dot{w}$ versus time, for calculated data from the GA (black line) and reference data from X-Plane (gray line) in three dimensions.

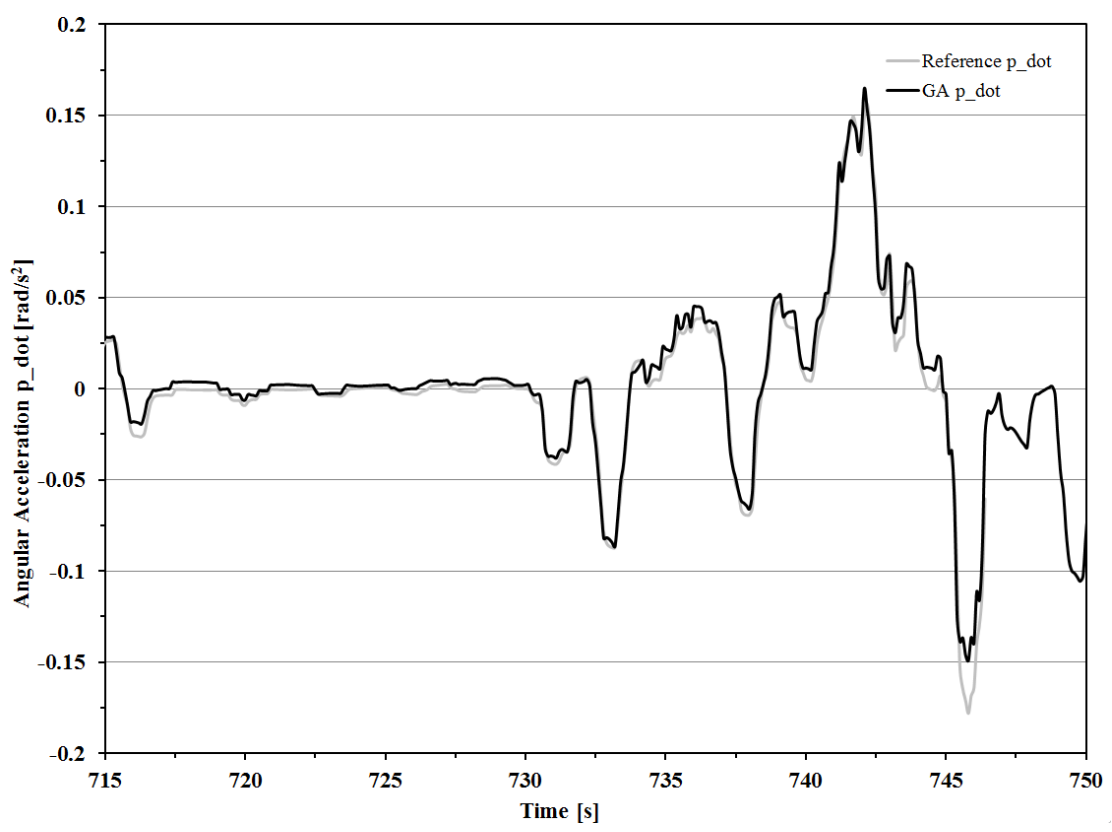

Figure 5.10: Plot of angular acceleration $\dot{p}$ versus time, for calculated data from the GA (black line) and reference data from X-Plane (gray line) in three dimensions. 


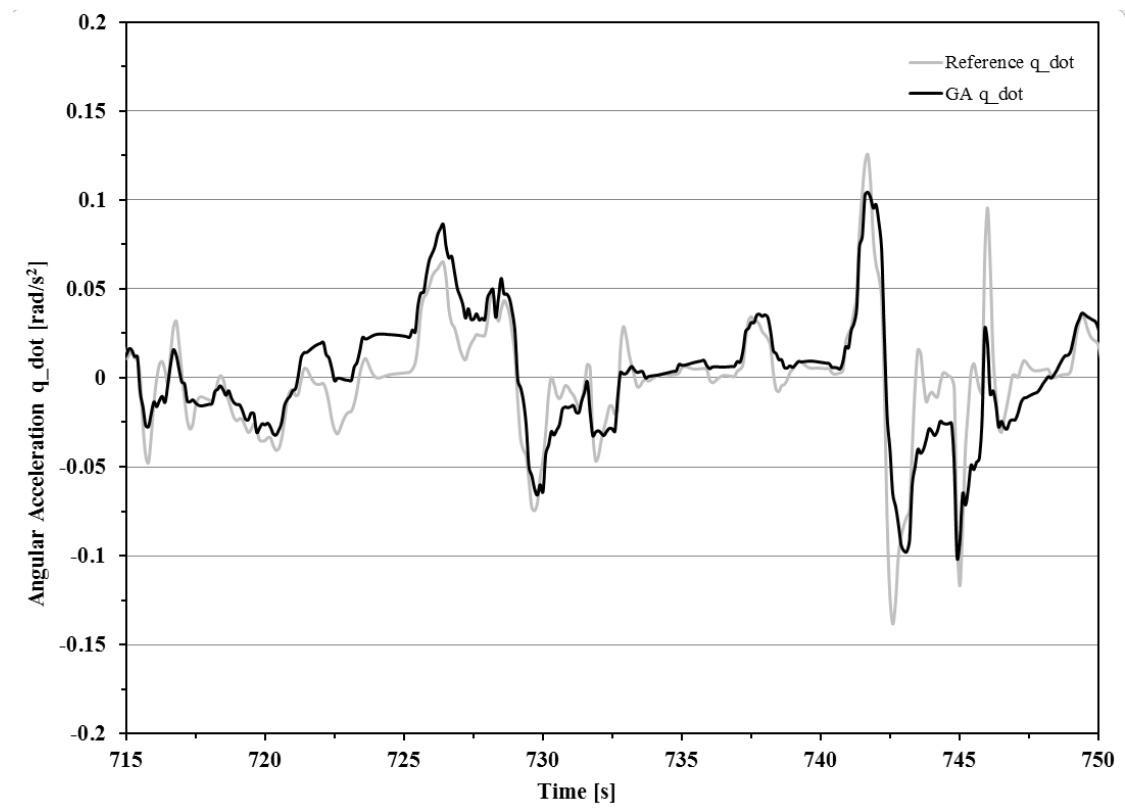

Figure 5.11: Plot of angular acceleration $\dot{q}$ versus time, for calculated data from the GA (black line) and reference data from X-Plane (gray line) in three dimensions.

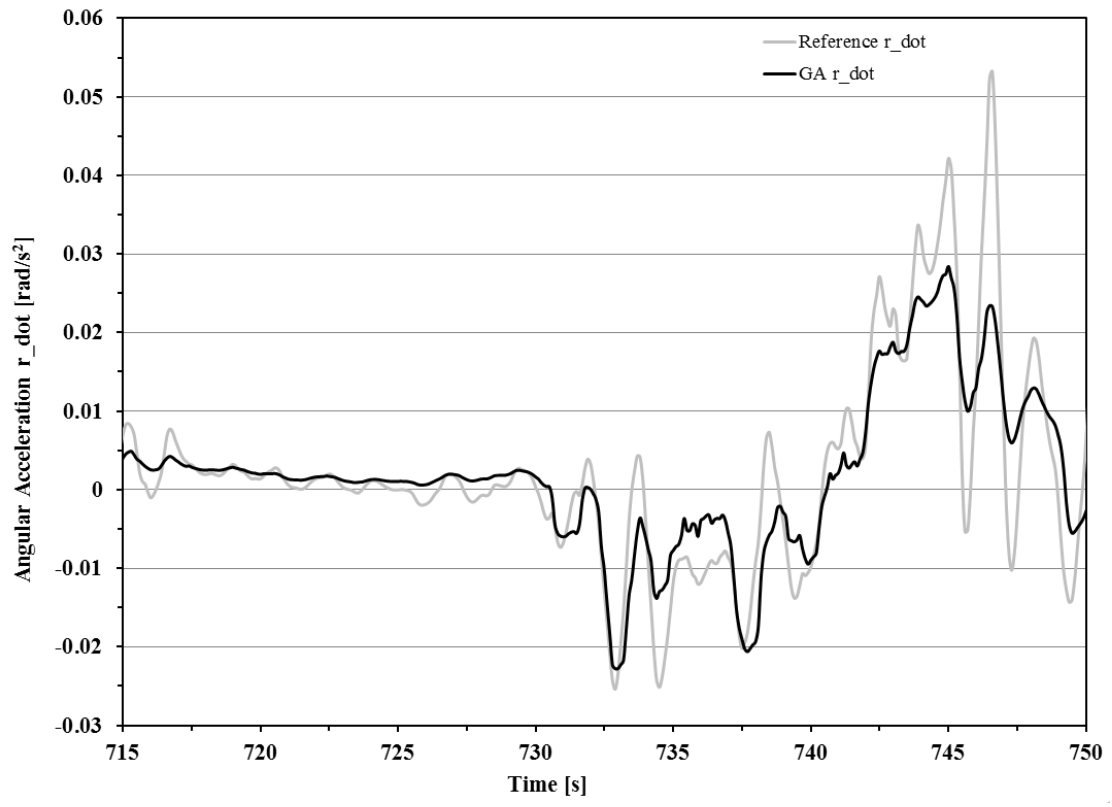

Figure 5.12: Plot of angular acceleration $\dot{r}$ versus time, for calculated data from the GA (black line) and reference data from X-Plane (gray line) in three dimensions. 
using a stability-derivative-based math model, differences in the data were inevitable due to the assumptions and omissions that are inherent in the stability derivative derivations.

Had Microsoft Flight Simulator software been a better fit for the KatanaSim project, the tuned stability derivatives could have been directly written into a performance file dictating aircraft behaviour. Unfortunately, Microsoft Flight Simulator is not compatible with most of the KatanaSim hardware. Because X-Plane calculates flight models based solely on geometry and power specifications, it was not possible to apply the tuned stability derivatives to the KatanaSim flight model. While the GA was proven to be an excellent tool for tuning stability derivatives, tuning a flight model for X-Plane would require a custom software plug-in in order to interface with the equations of motions used in the system in order to tune the geometric and power parameters of the flight model. This type of software plug-in, or the flight dynamics math model itself, would likely have to be provided by Laminar Research. This possibility can be investigated in the next phase of the KatanaSim project. Future work should include researching the possibility of implementing a different software package compatible with the KatanaSim hardware that would enable more control over the flight dynamics model.

In the absence of software enabling the application of a GA directly to X-Plane, the benefits of using a NN could need to be re-evaluated. The GA is known to be reliable, adaptable, and simple to implement but only when the mathematical model for the system is known. The potential ability of a neural network to identify the Plane Maker parameters without requiring knowledge of the model could allow for the precise tuning of a suitable KatanaSim flight model using the acquired flight test data. 


\section{Chapter 6}

\section{Conclusion}

\subsection{Contributions}

During the course of conducting the work described in this thesis, the following significant research and development contributions were made.

\section{KatanaSim Hardware}

The development of a cost-effective, high-fidelity, type-specific flight simulator began with the integration of COTS components and original aircraft parts into the KatanaSim fuselage. Specifically, the instrument panel was reverse engineered and modified in order to accommodate the installation of carefully selected COTS flight simulator gauges and avionics. The centre console assembly as well as the throttle and propeller speed levers were installed and a rotary-potentiometer-based system was integrated to detect pilot inputs to the levers and console controls. A simpleto-tune passive feedback system was implemented for the control column using a simple and cost-effective design using surgical tubing and turnbuckles. A section of the original brake system was installed in the cockpit and pressure sensors were connected to the lines in order to detect and transmit pilot inputs. 


\section{Flight Test Methodology}

Publicly-available Katana performance data is quite limited, though imperative for successful flight model tuning. This lack of information inspired the design of a minimally-intrusive test methodology which could be widely applied to any small aircraft but which was implemented using a Katana. An instrumentation interface board was prepared and mounted in the baggage compartment of the Katana. A flight control tracking method, using string potentiometers, was devised and installed in the cockpit and the required circuit board was constructed to facilitate power and data collection for the system. A Transport Canada approved battery and battery enclosure were selected for the system and an emergency procedure was written in case of a battery fire. An audio-visual recording system was installed by mounting a GoPro Hero 2 camera in the cockpit and tapping into the radio communications system of the aircraft.

Appropriate flight test manoeuvres were chosen as a group, but specifically as part of a contribution to this thesis, standard flight testing procedures for each specific manoeuvre were researched and implemented in the flight plan. The flight tests were carried out with step-by-step instructions being provided to the pilot during the flight, while manual data was recorded simultaneously.

3. Development and Analysis of a Flight Model

Another contribution to the project was the development of a flight model for use in the KatanaSim. The model was developed using information from a variety of sources including manuals from Diamond Aircraft, Type Certificate Data Sheets from Transport Canada and the FAA, and empirical measurements and observations. Modifications to the published data were made only to account for errors and contradictions in the published data. Of particular interest was the acquisition of 
the previously unpublished Wortmann FX63-137/20 airfoil profile coordinates using the Optotrak Coordinate Measurement Machine.

The directly resulting flight model did not match the performance data obtained from the flight test or from published sources, and so a sensitivity study was performed in order to assess the feasibility of iteratively tuning the model by identifying the effect of Plane Maker parameters on the performance of the X-Plane model. The sensitivity study showed the difficulties inherent with such an approach, and so a new tuning method for the flight model was designed.

\section{A Genetic Algorithm for Automated Flight Model Tuning}

A simple, yet adaptable genetic algorithm for parameter estimation was designed and and validated using a variety of dynamic problems including coupled and uncoupled systems. The linearized longitudinal rigid body equations of motion for an aircraft were developed using a stability-derivative-based approach. These equations were then used with the genetic algorithm to successfully identify 14 stability derivatives required for an aircraft to match the simulated flight performance data obtained from X-Plane. Having proved the ability of the algorithm to successfully identify the longitudinal stability derivatives, the algorithm was expanded and used to obtain 28 longitudinal, lateral, and directional stability derivatives in a fully-three-dimensional mathematical model. The algorithm once again successfully proved its ability to facilitate flight model tuning.

\subsection{KatanaSim Assessment}

This project was an investigation into the feasibility of developing a cost-effective, highfidelity, type-specific, small aircraft simulator for use as part of an ab-initio flight training program. The development of the KatanaSim proved the feasibility of the endeavour. 
Particularly successful was the high level of physical fidelity achieved by the use of original aircraft parts, most notably the Katana fuselage.

Not all of the integrated hardware systems reach the high-level of fidelity desired for the KatanaSim. The visual system, which is recognized as an interim solution only, provides an adequate display for training only the most basic manoeuvres. A more immersive visual system, such as the originally-proposed design using the aircraft canopy as the projection surface, could greatly increase the training effectiveness of the KatanaSim. The haptic cueing system, like the visual system, is an interim solution designed to provide basic cueing until dynamic feedback systems can be implemented.

One remaining hardware challenge stems from the reliability issues encountered with the simulated gauges and avionics installed in the instrument panel. While troubleshooting has been successful and only minor issues remain with gauge functionality, the quality of the gauges, and the high frequency with which customer support is required from the manufacturer, highlights the need for higher-quality gauges.

The use of X-Plane as the simulator software was, for the most part, an excellent choice. The open-source nature of the software, and the ease with which the majority of the hardware could be integrated into the system, enabled the use of many COTS and original aircraft components which could have otherwise been difficult. The bladeelement-theory-based flight model engine was initially considered as an advantage as it was capable of providing more accurate flight dynamics in edge-of-envelope manoeuvres and had a history of certification with the FAA. Unfortunately this lead indirectly to the greatest challenge encountered with the flight model: the inability to efficiently tune the flight model. Tuning is possible through a work-intensive approach of parametersensitivity investigations. Given the availability of the flight test data though, the model tuning process could be greatly improved by the availability of a method allowing the developed genetic algorithm to interface with the blade element modelling method used 
by X-Plane.

The cost of the simulator, as-built but not including the motion platform, is just $\$ 119$ 800, which is less than a quarter of the cost of the King Air Mechtronix flight training device currently used at OAS Flight Centre for ab-initio training. While the KatanaSim has not yet reached the level of fidelity as the Mechtronix flight training device, it still has immense potential for training ab-initio students.

\subsection{Future Work}

The reliability of the instruments should be improved to minimize the likelihood of new issues arising in the future. As COTS components are still the most favourable solution, additional research should be done to determine whether there exists, outside of Flight Illusion and SimKits, other well-reputed flight simulator instrument manufacturers. Inhouse design and manufacture of physical gauges should also be investigated, as should the feasibility of implementing a flat screen display behind the instrument panel and the implementation of instrument adjustment knobs with this system.

On a larger scale, the visual system of the KatanaSim should be taken to the next phase and a new, more immersive design should be implemented. Control loaders for active force feedback should be implemented, either using an in-house design or COTS components.

Work on the flight model should continue, with a particular focus on acquiring access to the X-Plane math model or else investigating other software packages that would be suitable for integration with the KatanaSim hardware while providing more user-access to the flight dynamics modelling aspect of the software. Further performance assessment of the genetic algorithm would be beneficial. The capability of the algorithm was proven, but additional testing can be done to prove the results are consistent over multiple runs and that convergence will still occur when using large amounts of actual flight test data. 
All of these system upgrades should be undertaken with eventual Transport Canada certification as an Flight Training Device in mind. Once the hardware and software have been upgraded, extensive testing of the KatanaSim systems should be performed to prove that the level of system fidelity required by Transport Canada has been achieved.

The progress so far on the admittedly ambitious KatanaSim project has been quite astounding and the feasibility of such a project has been proven. The continuation of this work in future will undoubtedly lead to a certified flight training device that could markedly improve the ab-initio flight training received by student pilots. 


\section{References}

[1] R. Page. Brief History of Flight Simulation. In Proceedings of SimTecT 2000, pages $1-11,2000$.

[2] M. Lansdaal, L. Lewis, and W. Bezdek. The History of Commercial Simulators and the Boeing 777 Systems Integration Lab. In Collection of Technical Papers - AIAA Modeling and Simulation Technologies Conference, volume 2, pages 594-607, 2004.

[3] J. M. Rolfe. Flight simulator research at the Royal Air Force Institute of Aviation Medicine. Applied Ergonomics, 4(2):84-90, June 1973.

[4] M. D. White, P. Perfect, G. D. Padfield, A. W. Gubbels, and A. C. Berryman. Acceptance Testing and Commissioning of a Flight Simulator for Rotorcraft Simulation Fidelity Research. Proceedings of the Institution of Mechanical Engineers, Part G: Journal of Aerospace Engineering, 227(4):663-686, March 2012.

[5] N. D. Macchiarella, T. Brady, and P. K. Arban. High Fidelity Flight Training Devices in the Training of Ab Initio Flight Students. 24th Digital Avionics Systems Conference, 1, 2005.

[6] PanAM International Flight Academy. www.flightexperience.com/training.

[7] H. Swaine. Personal communication with retired airline captain.

[8] CAE. www.cae.com, Accessed March 2014. 
REFERENCES

[9] N. D. Macchiarella, P. K. Arban, and S.M. Doherty. Transfer of Training from Flight Training Devices to Flight for Ab-Initio Pilots. International Journal of Applied Aviation Studies, 6(2):299 - 314, 2006.

[10] S. Swaine, J. Plumpton, M. J. Leveille, and R. Langlois. Feasibility Assessment of a Cost-Effective Type-specific Small Aircraft Simulator. In Canadian Society for Mechanical Engineering International Congress, 2012.

[11] Aeroplane and Rotorcraft Simulator Manual: TP 9685E. Transport Canada, 2nd edition, January 1998.

[12] T. M. Kirton. Integrating Level 6 Flight Training Devices into Flight Training Programs at the Beginning Pilot Level: Challenges and Opportunities. Proceedings. The 21st Digital Avionics Systems Conference, 2, 2002.

[13] N. D. Macchiarella. Advancements in Flight Training Devices for Ab Initio Pilot Use. In AIAA Modeling and Simulation Technologies Conference and Exhibit, number August, pages 1-9, 2008.

[14] Z. Shutao, H. Jingfeng, H. Qitao, and H. Junwei. A Low-Cost PC-Based Flight Simulator Development. 2008 2nd International Symposium on Systems and Control in Aerospace and Astronautics, 2008.

[15] Z. Lei, J. Hongzhou, and L. Hongren. PC Based High Quality and Low Cost Flight Simulator. 2007 IEEE International Conference on Automation and Logistics, 2007.

[16] Diamond Aircraft Industries. DA20-A1 Aircraft Flight Manual Rev. 18. 2012.

[17] J. Plumpton, S. Swaine, S. Abraham-Doman, R. Langlois, C. Merrett, and J. Laliberte. Carleton University Katana Flight Test Plan, Report No. ADL/13/JP/1.

[18] Diamond Aircraft Industries. DA20-A1 Aircraft Maintenance Manual. 
REFERENCES

[19] From The Ground Up. Aviation Publishers Co., 28th edition, 2000.

[20] Flight Illusion. www.flightillusion.com, Accessed March 2014.

[21] TRC Simulators. Simkits. www.simkits.com, Accessed March 2014.

[22] J. Plumpton. Development of a Full Flight Simulator for Ab-Initio Flight Training with Emphasis on Hardware Interfacing and Motion Integration. Master's thesis, Department of Mechanical and Aerospace Engineering, Carleton University, 2014.

[23] Futureshop. www.futureshop.ca, Accessed March 2014.

[24] Omega. www.omega.ca, Accessed March 2014.

[25] Arduino. www.arduino.cc, Accessed March 2014.

[26] Microsoft Game Studios. www.microsoft.com/games/fsinder, Accessed March 2014.

[27] Laminar Research. X-plane 10. www.x-plane.com, Accessed March 2014.

[28] Laminar Research. Plane Maker Manual. http://www.xplane.com/files/manuals/Plane_Maker_manual.pdf, 2013.

[29] D. G. Mitchell, R.H. Hoh, A Atencio J. R., and D. L. Key. Ground Based Simulation Evaluation of the Effects of Time Delays and Motion on Rotorcraft Handling Qualities. Technical report, US Army Aviation Systems Command, Ames Research Centre, 1992.

[30] R. A. Hess. Modeling the Effects of Display Quality upon Human Pilot Dynamics and Perceived Vehicle Handling Qualities. IEEE Transactions on Systems, Man, and Cybernetics, 25(2), 1995. 
REFERENCES

[31] S.D. Beard, S. E. Reardon, E. L. Tobias, and B. L. Aponso. Simulation System Fidelity Assessment at the Vertical Motion Simulator. In American Helicopter Society (AHS) Annual Forum and Technology Display; Phoenix, AZ; 21-23 May 2013; United States, pages 1-8, 2013.

[32] Ralph D. Kimberlin. Flight Testing of Fixed Wing Aircraft. AIAA Education Series. Smerican Institute of Aeronautics and Astronautics, Inc.

[33] Transport Canada. Canadian Aviation Regulations, Part V, Standard 571: Appendix A.

[34] Diamond Aircraft Industries. DA20-A1 Illustrated Parts Catalogue.

[35] J. Auld and K. Srinivas. Aerodynamics for Students: Compressible Flow. www.aeromech.usyd.edu.au/aero/gasdyn/node1.html, Accessed March 2014.

[36] MH-AeroTools. Javafoil. www.mh-aerotools.de/airfoils/javafoil.htm, Accessed March 2014.

[37] M. Gautier, A. Janot, and P. Vandanjon. A New Closed-loop Output Error Method for Parameter Identification of Robot Dynamics. Control Systems Technology, IEEE Transactions on, 21(2):428-444, 2013.

[38] J. Lee and S. Yoon. Application of Parameter Estimation to FTD for a Light Airplane via Flight Tests. Simulation Modelling Practice and Theory, 15(6):690-702, July 2007.

[39] T. Söderström and P. Stoica. Some Properties of the Output Error Method. Automatica, 18(1):93-99, 1982.

[40] G. Chowdhary and R. Jategaonkar. Aerospace Science and Technology, March.

[41] R. E. Marks and Hermann Schnabl. Genetic Algorithms and Neural Networks: A Comparison Based on the Repeated Prisoners Dilemma. 1998. 
REFERENCES

[42] V. Lekshmisree, D. Karthikeyan, S. Ushakumari, and V. Lalithambika. A Neural Network-based Approach for Parameter Estimation of Flight Vehicle. 2011.

[43] P. Koehn. Combining Genetic Algorithms and Neural Networks: The Encoding Problem. Master's thesis, University of Tennessee, Knoxville, 1994.

[44] T. Rajkumar and J. Bardina. Prediction of Aerodynamic Coefficient Using Genetic Algorithm Optimized Neural Network for Sparse Data. 2002.

[45] G. Valmorbida, W. Lu, and F. Mora-Camino. A Neural Approach for Fast Simulation of Flight Mechanics. 38th Annual Simulation Symposium, 2005.

[46] M. Gulsen, A. E. Smith, and D. M. Tate. A Genetic Algorithm Approach to Curve Fitting, 1995.

[47] H. Xu and L. Jin. Design of three-axis flight simulator controller based on genetic algorithm. In Intelligent Control and Automation, 2004. WCICA 2004. Fifth World Congress on, volume 6, pages 5430-5434. IEEE, 2004.

[48] S. Yurt, I. Özkol, M. Kaya, and C. Haciyev. Optimization of the PD Coefficient in a Flight Simulator Control via Genetic Algorithms. Aircraft Engineering and Aerospace Technology, 74(2):147-153, 2002.

[49] D. A. Caughey. Introduction to Aircraft Stability and Control Course Notes.

[50] R. C. Nelson. Flight Stability and Automatic Control. 2nd edition.

[51] Darwin's Theory of Evolution - The Premise. http://www.darwins-theory-ofevolution, Accessed March 2014.

[52] A. Eiben and J Smith. Introduction to Evolutionary Computing: Genetic Algorithms. 
Appendix A

Quotation from Servos and

Simulation, Inc. for a Haptic

Feedback System 


\section{Seryos \\ Simulation, Inc. \\ HIGH TECHNOLOGY ENGINEERING}

January 10, 2013

Suzanne Swaine

Master of Applied Science Candidate

Mechanical and Aerospace Engineering

Carleton University

Phone: 6135202600 x 4590

\section{Proposal for a 400-X High Force EDCL System:}

1. Three Axis System

$\$ 65,000.00$ USD

2. Computer System and Software $\$ 10,000.00$ USD

System Total $\$ 75,000.00$ USD

\section{Integration:}

The price above does not include on-site integration. If you require integration on site, we typically do this on a separate contract and bid it out.

\section{Payment Terms:}

Typically, we accept $50 \%$ down payment, $50 \%$ upon shipment. Shipping is FOB our dock in Longwood, Florida. We do provide the shipping container in the price of the system. We cannot start on the contract until a purchase order is in place and monies have arrived please.

\section{Timing and Delivery:}

We will endeavor to get the system delivered as soon as possible - 45 to 60 days is typically required for a single system.

If there are any questions or concerns that need to be addressed, please call or email me as soon as possible. We look forward to your business and hope that you have a wonderful day.

Sincerely,

Rachel Baker rbaker@servos.com Engineering Dept 


\section{Appendix B}

\section{Battery Fire Procedure}

1. Master Switch : OFF

2. Cabin Air: Open

3. Cabin Heat: Closed

4. Fire Extinguisher: Deploy as required

5. Douse battery with water to cool

6. Land as soon as possible 
Appendix C

Flight Permit 
To. A :

Ottawa Aviation Services

\section{Hunt Club Rd. Ottawa, ON, K1V 1 C1}

\begin{tabular}{|c|c|c|c|c|}
\hline $\begin{array}{l}\text { Nationality and } F \\
\text { Marques de national }\end{array}$ & $\begin{array}{l}\text { gistration Marks } \\
\text { et d'immatriculation }\end{array}$ & $\begin{array}{l}\text { Aircraft Manufa } \\
\text { Constructeur et } m\end{array}$ & $\begin{array}{l}\text { cturer and Model } \\
\text { nodèle de l'aéronef }\end{array}$ & $\begin{array}{c}\text { Aircraft Serial Number } \\
\text { Numéro de série de l'aéronef }\end{array}$ \\
\hline Canadian & C-EWSH & DIAMONI & DA2OA1 & 10079 \\
\hline
\end{tabular}

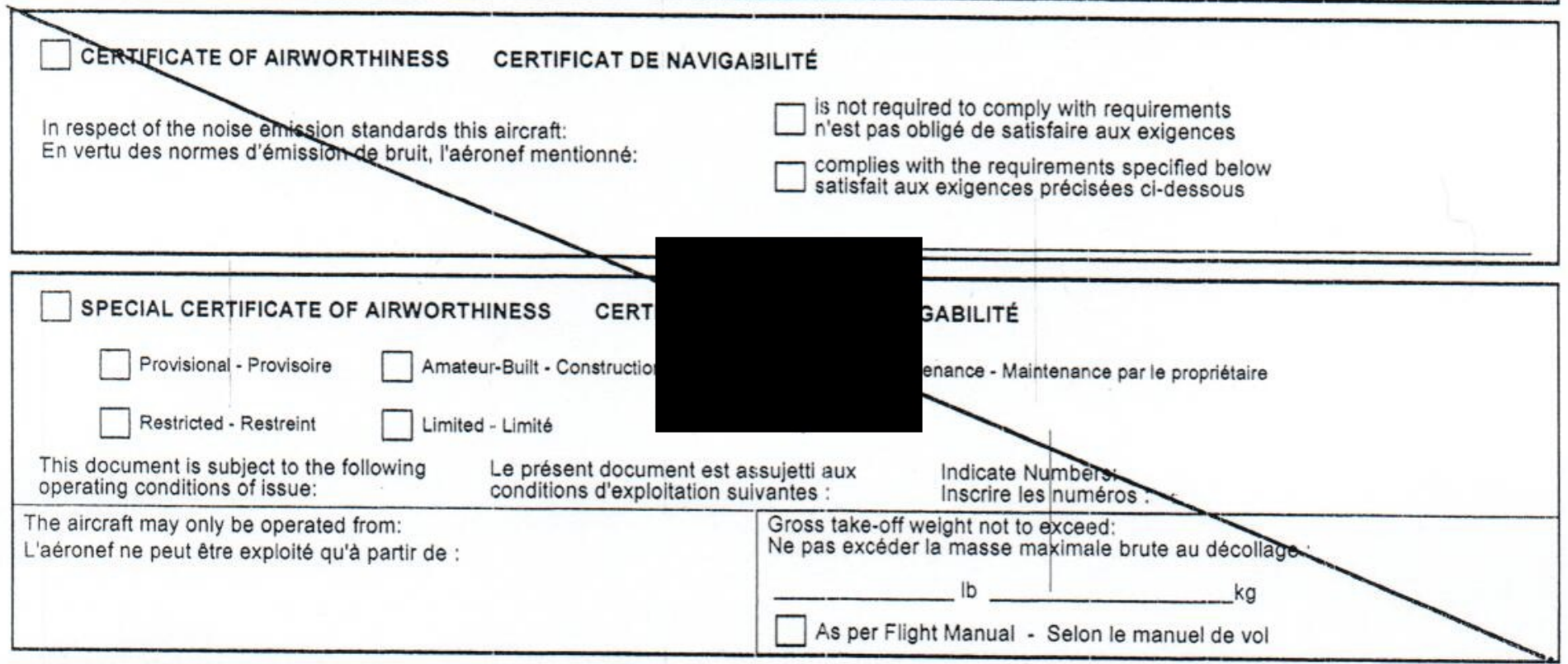

Flight Permit - Specific Purpose

Permis de vol . Fin Spécifique

$\square$ Ferry Flight

Vol de convoyage

Importation or exportation flight

Vol pour fin d'importation ou d'exportation

Other temporary purposes (Specify)

Pour d'autres fins temporaires (Préciser)
Flight Permit - Experimental

Permis de vol - Expérimental

Demonstration, market survey or crew training

Vol de démonstration, étude de marché ou formation d'équipage

$$
\text { Flight from - Vol de }
$$

Ottawa International

CYOW

Test Flight IAW Data Package $\mathrm{ADL} / 13 / \mathrm{JP} / 1$

\section{Test Flight}

50 Nautical Miles

Le present document est assujetti aux conditions d'exploitation suivantes
Indicate Numbers:

Inscrire les numeros: $1,2,4,7,10$ operating conditions of issue

The aircraft may only be operated from:

L'aéronef ne peut être exploité qu'à partir de :

As Indicated above

Gross take-off weight not to exceed:

Ne pas excéder la masse maximale brute au décollage :

$$
1609 \mathrm{lb}
$$

As per Flight Manual - Selon le manuel de

This document is valid for the number of days indicated on the Le present document reste valide pendant le nombre de jours right, following the date of issue. Where pertinent, a replacement indiqués à droite qui suivent la date de délivrance. S'il y a flight authority will he issued to vou_ TCR-198 lieu, ühe autorite de vol de remplacement vous sera délivré.

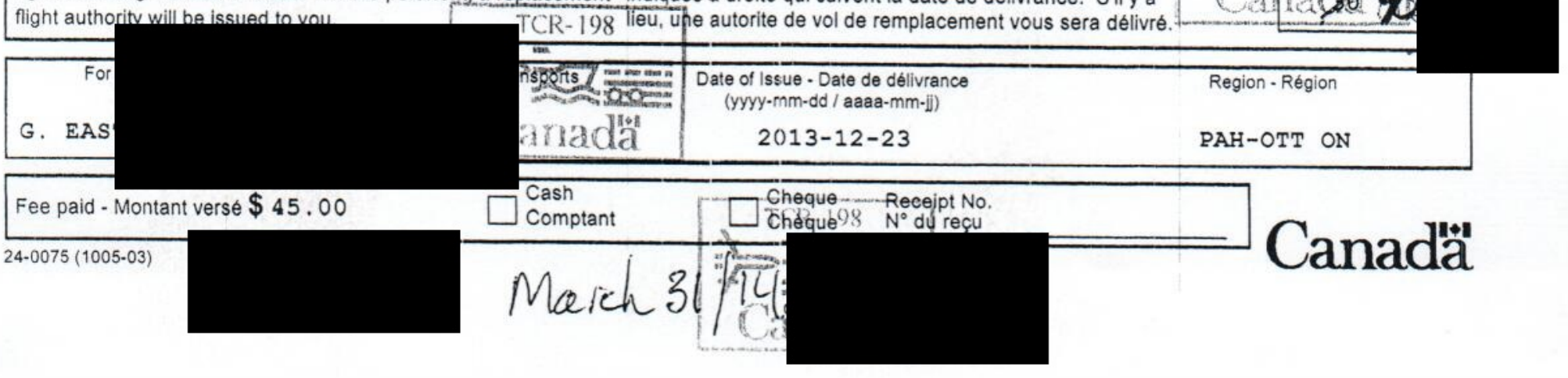




\section{Operating Conditions}

1. Valid for the purpose of (specify purpose);

2. Use as a commercial aircraft prohibited;

3. Crew members only, no passengers:

4. Crew members only - no passengers, except those persons whom the pilot-in-command determines as having a bona fide interest in the demonstration;

5. Crew members shall be the holders of valid and subsisting pilot licences issued or endorsed by Canada or the (state of registry to be specified) and which are appropriate to their duties;

6. Gross take-off weight not to exceed (specific weight to be listed on the flight permit);

7. Flight into known or predicted icing conditions prohibited;

8. VNE to be established by flight test:

9. Day VFR only:

10. VFR only:

11 Flight over built-up areas prohibited;

12. Flight over built-up areas prohibited, and flight in congested airspace to be avoided:

13. Flight over built-up areas prohibited except during take-offs and landings:

14. Flight authority issued by (specify authority) shall be valid and shall be carried on board the aircraft together with this validation;

15. Controlling Air Traffic Control unit to be informed of the experimental nature of the aircraft and the evaluation program prior to flight;

16. The aircraft shall be formally or provisionally registered in (specify state);

17. Compliance required with the conditions on the (specify type of permit and authority);

18. Controlling Agency at airport of take-off shall be informed of overload conditions prior to take-off;

19. Permission of the foreign aviation authority required prior to flight in their airspace;

20. The aircraft can only operate from a base indicated by Transport Canada in order to provide the highest degree of safety for the operation of the aircraft;

21. The aircraft shall not be operated (flown) more than 25 nautical miles from the base mentioned in (20) except with written authority of the Regional Director Aviation Licensing, (specify region) Region, which will be provided, taking into account the safety of the flight;

22. The aircraft shall not be flown over any built-up area, or open air assembly of persons;

23. Carriage of persons other than for dual instruction is prohibited (not to be used for single seat aircraft);

24. Aerobatic flight is prohibited (not to be used for balloons);

25. During the first 5 hours of flight, the aircraft can only be flown by pilots who have acquired not less than 100 hours of pilot-in-command flight time in powered aircraft (not to be used for gliders, gyroplanes, or balloons);

26. Aircraft is to be registered for "Private Purposes" only;

27. Aircraft to be placarded in the cockpit "Restricted - Agricultural Purposes Only":

28. Validity period;

29. Flight testing to be conducted away from built-up areas, airways and air routes:

30. Ferry-flight (specify from) to (specify to) to be via (specify routing) with technical landings as required;

31. The side of the aircraft fuselage is to be placarded, in a place that is readily visible to persons entering the aircraft, in letters at least $3 / 8$ inch in height and of a colour that contrasts sharply with the background on which they are shown, in both official languages, as follows:

NOTICE: THIS AIRCRAFT IS OPERATING WITHOUT A CERTIFICATE OF: AIRWORTHINESS.

AVIS : CET AÉRONEF VOLE SANS CERTIFICAT DE NAVIGABILITÉ.

\section{Conditions d'exploitation}

1. Valide aux fins de (préciser les fins);

2. L'exploitation à titre d'aéronef commercial est interdite

3. Membres d'equipage seulement - pas de passagers;

4. Membres d'équipage seulement - pas de passagers, sauf les personnes qui de l'avis du commandant de bord ont un intérêt réel dans la démonstration;

5. Les membres d'équipage doivent être titulaires de licences de pilote valides et en vigueur délivrées ou annotées par le Canada ou (préciser l'État d'immatriculation) et correspondant à leurs fonctions.

6. Ne pas excéder la masse maximale brute au décollage (qui doit être indiquée sur le permis de vol);

7. Vol interdit dans des conditions de givrage existantes ou prévues;

8. La VNE doit être établie par essai en vol;

9. VFR de jour seulement;

10. VFR seulement;

11. Le survol des zones bâties est interdit;

12. Le survol des zones báties est interdit, et le vol dans un espace aérien à forte densité de circulation est à éviter;

13. Le survol des zones bâties est interdit, sauf au décollage et à l'atterrissage;

14. L'autorité de vol délivrée par (préciser l'autorité) doit être en vigueur et se trouver à bord de l'aéronef avec la présente validation;

15. L'unité de contrôle de la circulation aérienne qui exerce le contrôle doit être informée avant le vol de la nature expérimentale de l'aéronef et du programme d'évaluation;

16. L'aéronef doit être officiellement ou provisoirement immatriculé dans (préciser l'État):

17. La conformité avec les conditions figurant sur le (préciser le type de permis et l'autorité) est obligatoire;

18. L'organisme qui exerce le contrôle à l'aéroport de décollage doit être informé avant le décollage des conditions de surcharge;

19. Le vol dans l'espace aérien étranger est interdit, sauf avec l'autorisation préalable de l'autorité de l'aviation civile étrangère en cause;

20. L'aéronef ne peut être exploité qu'à partir de la base précisée par Transports Canada de façon à garantir le degré optimal de sécurité d'exploitation de l'aéronef;

21. L'aéronef ne peut être exploité que dans une zone d'un rayon maximum de $25 \mathrm{NM}$ de la base mentionnée a l'alinéa 20 , sauf avec l'autorisation écrite du directeur régional de la navigabilité, région (préciser la région), qui sera fournie compte tenu de la sécurité du vol:

22. Il est interdit de survoler des zones bâties ou des rassemblements en plein air;

23. II est interdit de transporter des personnes sauf pour l'instruction en double commande (ne pas utiliser dans le cas des aéronefs monoplaces);

24. Le vol d'acrobatie aérienne est interdit (ne pas utiliser dans le cas de ballons);

25. Seul un pilote ayant accumulé au moins 100 heures de vol à titre de commandant de bord d'aéronefs propulsés par un organe moteur est autorisé piloter cet aéronef au cours des cinq premières heures de vol (ne pas utiliser dans le cas des planeurs, des autogires ou des ballons):

26. L'aéronef doit être immatriculé " à des fins privées " seulement;

27. Une affichette "Restreint - fins agricoles seulement " doit être apposée dans le poste de pilotage;

28. Période de validité;

29. Les essais en vol doivent être effectués hors des zones báties, des voies aériennes et des routes aériennes;

30. Le vol de convoyage doit être effectue de (préciser la partance) à (préciser la destination) via (préciser la route) avec escales techniques au besoin;

31. Une affichette doit être apposée au côté du fuselage de l'aéronef, en un endroit facilement visible pour les personnes qui montent dans l'aéronef, en lettres d'au moins $3 / 8$ pouce de hauteur et d'une couleur contrastant clairement avec le fond sur lequel elles sont apposées, dans les deux langues officielles, portant les mots : 
Appendix D

Raw Data Sheets from Flight Tests 


\section{A Flight Test Plan - Flight 112 OS P $\mu$}

Pre-flight checks

\begin{tabular}{|c|c|}
\hline \multicolumn{2}{|c|}{ Take-off Holding Brakes } \\
\hline Wind Speed (kts) & 360 \\
\hline Wind Direction \\
\hline \multicolumn{2}{|c|}{ Pre-Takeoff Check } \\
\hline Bring aircraft to a halt at start of runway \\
\hline \multicolumn{2}{|c|}{ Apply full brakes } \\
\hline Apply full power and allow it to stabilize \\
\hline \multicolumn{2}{|c|}{ Event \# } \\
\hline \multicolumn{2}{|c|}{2} \\
\hline Engine (RPM) & 29 \\
\hline MP (in. Hg) & 24 SO \\
\hline Release brakes and use normal climb out \\
\hline Event \# (Wheels Up) & 3 \\
\hline Climb Speed (kts) & 65 \\
\hline
\end{tabular}

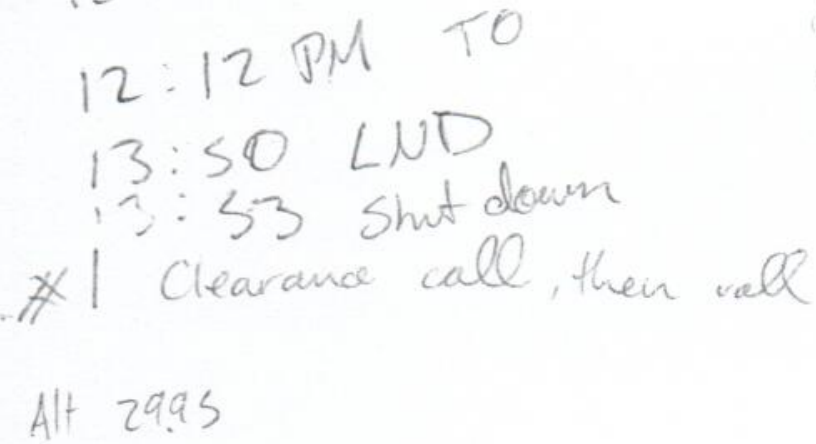

Climb out, then transit to Practice Area

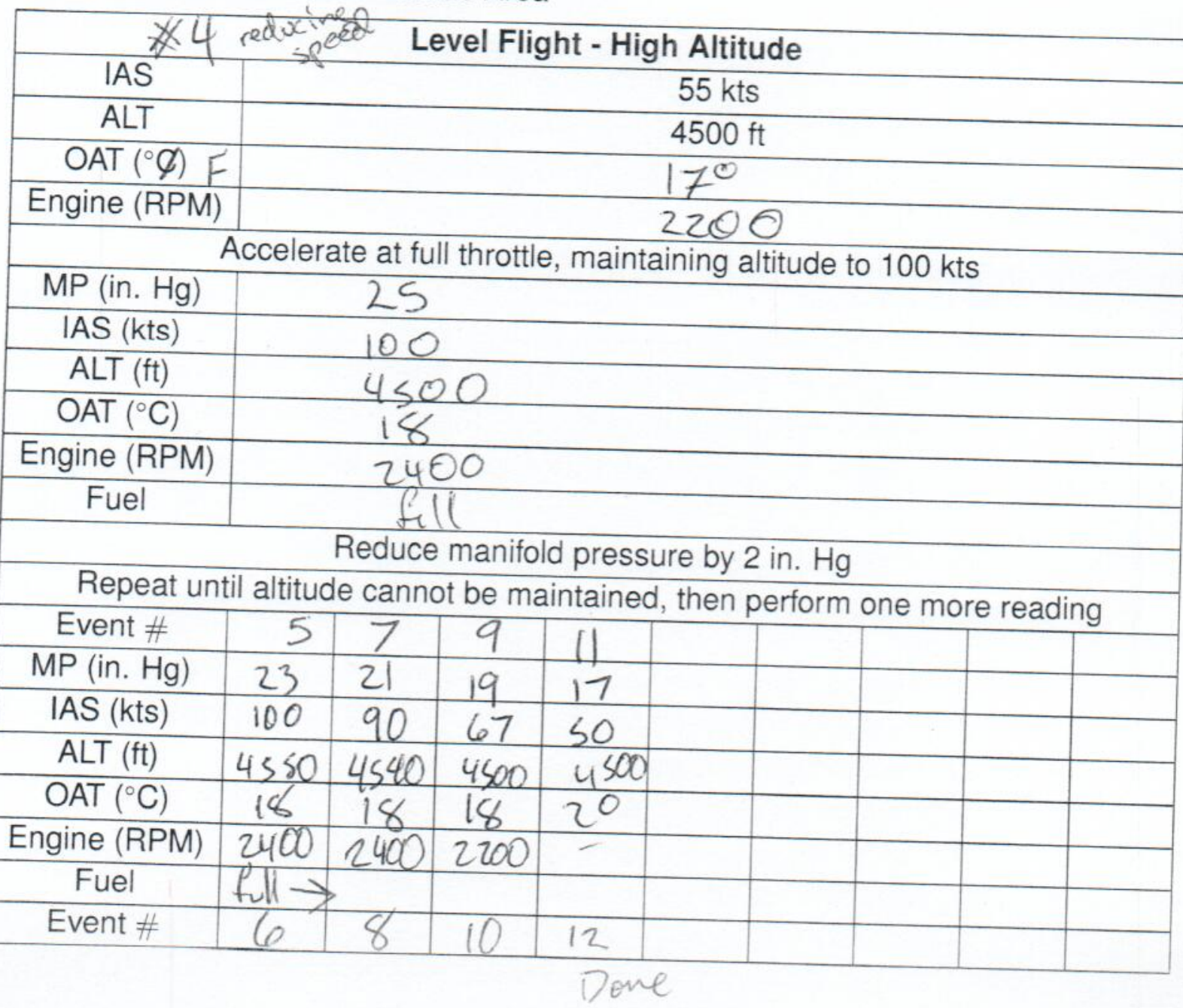




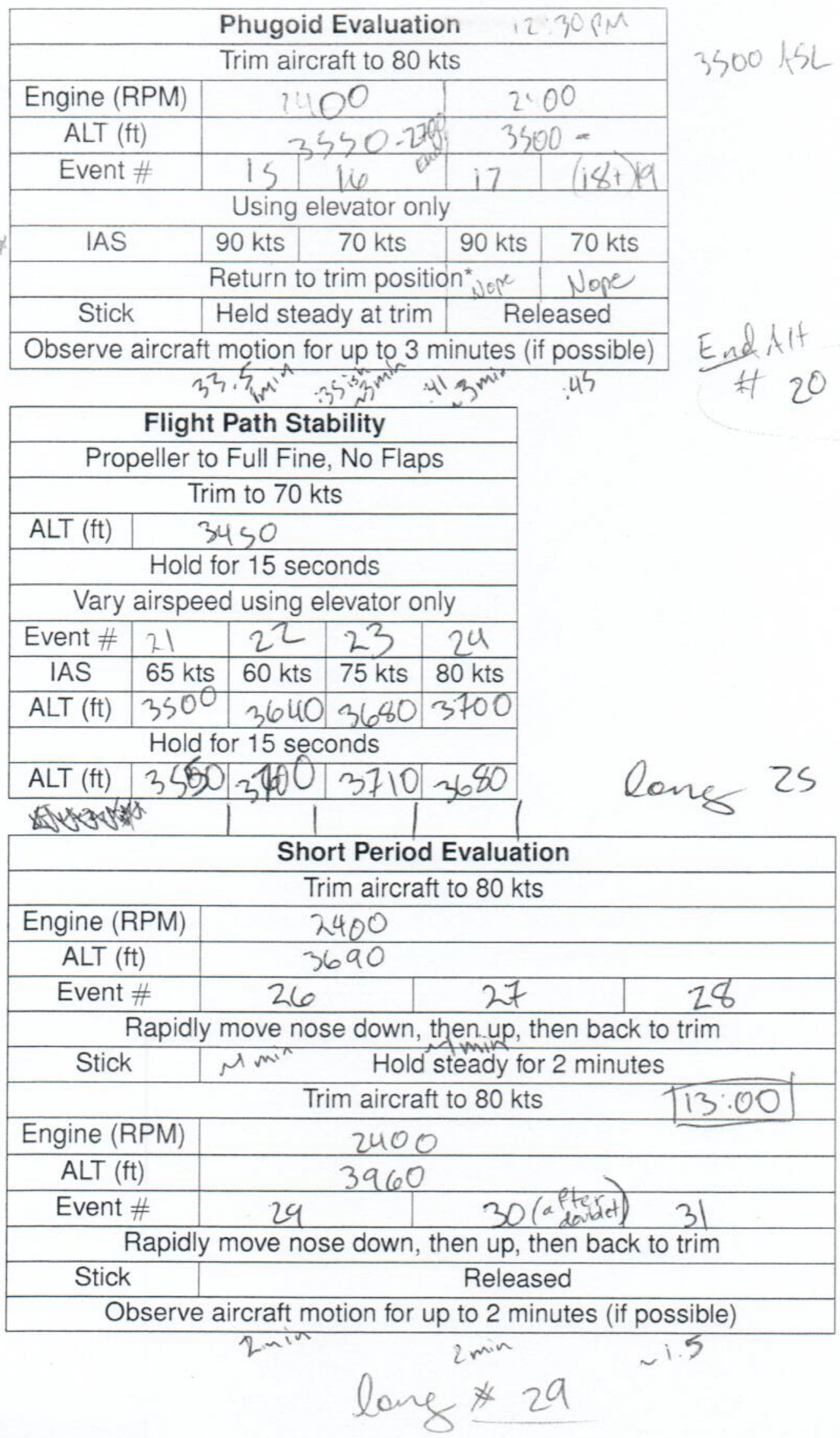

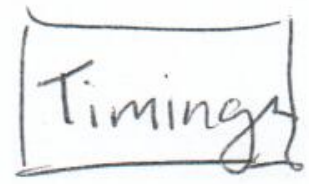




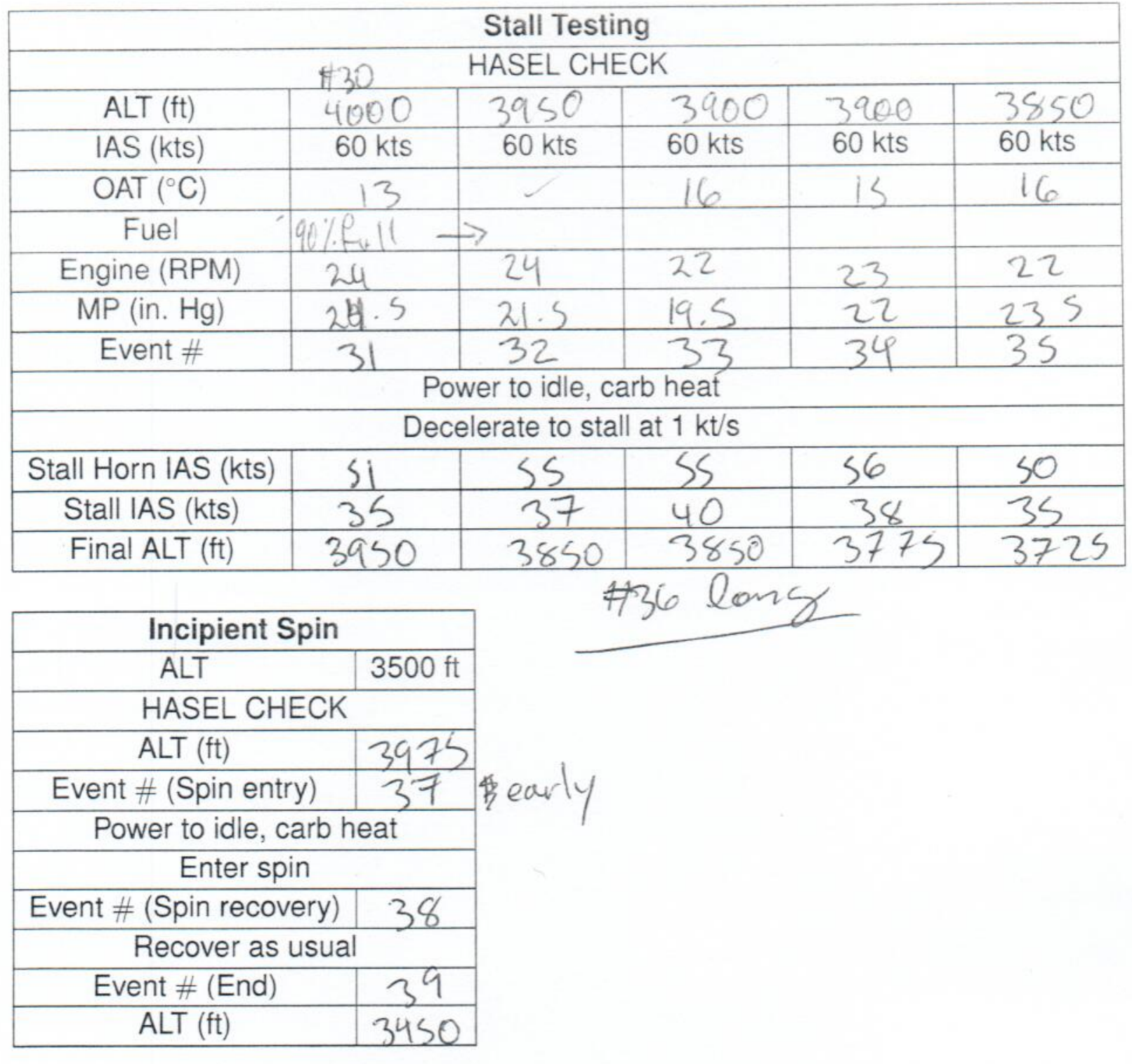

\begin{tabular}{|c|c|}
\hline \multicolumn{2}{|c|}{ Fully Developed Spin } \\
\hline ALT & $-4500 \mathrm{Tt}$ \\
\hline \multicolumn{2}{|c|}{ HASEL CHECK } \\
\hline $\mathrm{ALT}(\mathrm{ft})$ & 4000 \\
\hline Event \# (Spin entry) & 40 \\
\hline \multicolumn{2}{|c|}{ Power to idle, carb heat } \\
\hline \multicolumn{2}{|c|}{ Enter spin } \\
\hline Event \# (Spin recovery) & 41 \\
\hline \multicolumn{2}{|l|}{ Recover as usual } \\
\hline Event \# (End) & 3450 \\
\hline $\mathrm{ALT}(\mathrm{ft})$ & 42 \\
\hline
\end{tabular}




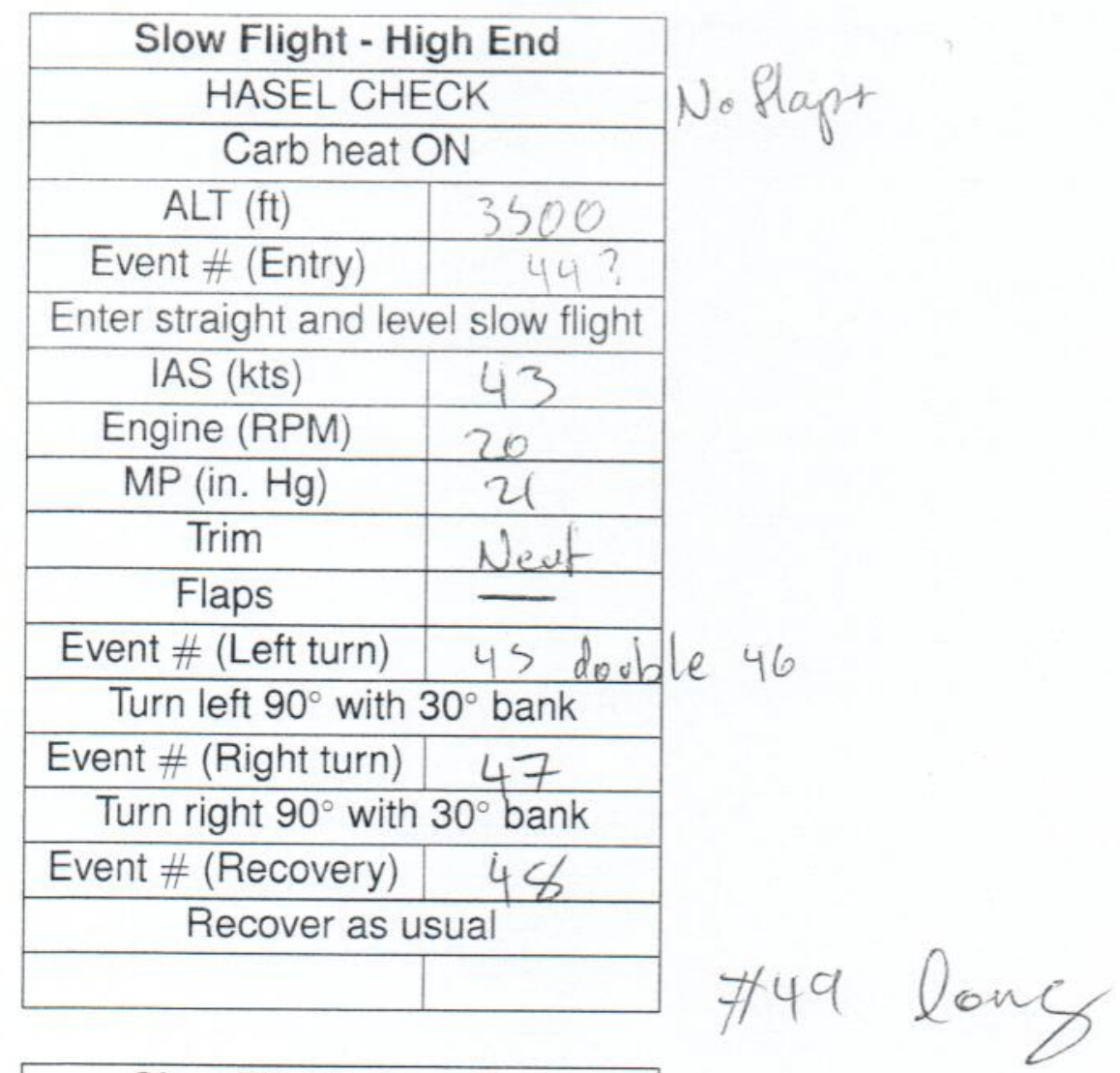

\begin{tabular}{|c|c|}
\hline \multicolumn{2}{|c|}{ Slow Flight - Low End } \\
\hline $\mathrm{ALT}(\mathrm{ft})$ & 3500 \\
\hline Event \# (Entry) & 50 \\
\hline \multicolumn{2}{|c|}{ Enter straight and level slow flight } \\
\hline IAS $(k t s)$ & 50 \\
\hline Engine (RPM) & 2050 \\
\hline MP (in. $\mathrm{Hg}$ ) & 22 \\
\hline Trim & New \\
\hline Flaps & - \\
\hline Event \# (Left turn) & 51 \\
\hline \multicolumn{2}{|c|}{ Turn left $90^{\circ}$ with $30^{\circ}$ bank } \\
\hline Event \# (Right turn) & 32 \\
\hline \multicolumn{2}{|c|}{ Turn right $90^{\circ}$ with $30^{\circ}$ bank } \\
\hline Event \# (Recovery) & 53 \\
\hline \multicolumn{2}{|c|}{ Recover as usual } \\
\hline & \\
\hline
\end{tabular}


A FLIGHT TEST PLAN - FLIGHT 1

20

\begin{tabular}{|c|c|}
\hline \multicolumn{2}{|c|}{ Spirals } \\
\hline ALT & $3500 \mathrm{ft}$ \\
\hline Engine (RPM) & 2400 \\
\hline MP (in. Hg) & 25 \\
\hline \multicolumn{2}{|c|}{ HASEL CHECK } \\
\hline ALT (ft) & 3500 \\
\hline Event \# (Spiral entry) & 55 \\
\hline \multicolumn{2}{|c|}{ Enter spiral } \\
\hline Event \# (Spiral recovery) & 56 \\
\hline Recover as usual (power idle, arb heat ON) \\
\hline Event \# (End) & 57 \\
\hline ALT (ft) & 3050 \\
\hline
\end{tabular}

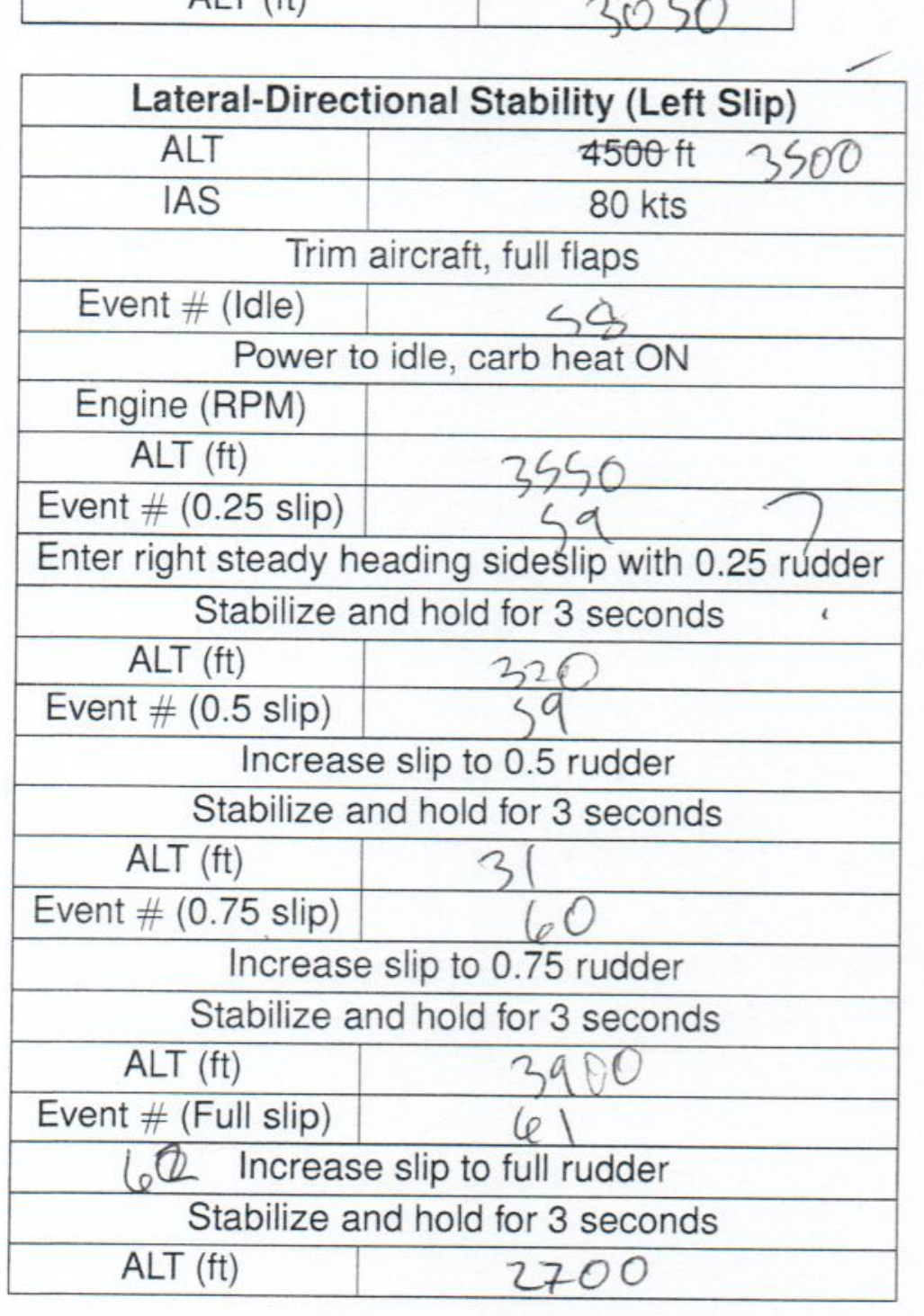

63

lang N/A 


\begin{tabular}{|c|c|c|}
\hline \multicolumn{3}{|c|}{ Lateral-Directional Stability (Right Slip) } \\
\hline ALT & $4500 \mathrm{ft}$ & 35 \\
\hline IAS & $80 \mathrm{kts}$ & \\
\hline \multicolumn{3}{|c|}{ Trim aircraft, full flaps } \\
\hline Event \# (Idle) & 3 & \\
\hline \multicolumn{3}{|c|}{ Power to idle, carb heat ON } \\
\hline Engine (RPM) & \multicolumn{2}{|l|}{1250} \\
\hline $\mathrm{ALT}(\mathrm{ft})$ & \multicolumn{2}{|l|}{3550} \\
\hline Event \# (0.25 slip) & \multirow{2}{*}{\multicolumn{2}{|c|}{$\frac{2}{2 g}$ sideslip with 0.25 rudder }} \\
\hline \multirow{2}{*}{\multicolumn{2}{|c|}{ Enter right steady heading sideslip with 0.25 rudder }} & \\
\hline \multirow{2}{*}{\multicolumn{3}{|c|}{$\begin{array}{l}\text { Stabilize and hold for } 3 \text { seconds } \\
\text { ALT (ft) }\end{array}$}} \\
\hline & & \\
\hline \multicolumn{3}{|c|}{ Event \# (0.5 slip) } \\
\hline \multicolumn{2}{|c|}{ Increase slip to 0.5 rudder } & 3 \\
\hline \multicolumn{3}{|c|}{ Stabilize and hold for 3 seconds } \\
\hline $\mathrm{ALT}(\mathrm{ft})$ & \multicolumn{2}{|c|}{3300} \\
\hline Event \# (0.75 slip) & & 4 \\
\hline \multicolumn{3}{|c|}{ Increase slip to 0.75 rudder } \\
\hline \multicolumn{3}{|c|}{ Stabilize and hold for 3 seconds } \\
\hline $\mathrm{ALT}(\mathrm{ft})$ & 3000 & \\
\hline Event \# (Full slip) & & 5 \\
\hline \multicolumn{3}{|c|}{ Increase slip to full rudder } \\
\hline \multicolumn{3}{|c|}{ Stabilize and hold for 3 seconds } \\
\hline ALT $(\mathrm{ft})$ & 2800 & \\
\hline
\end{tabular}

Transit to YOW

$$
70 \operatorname{lon} y
$$




\begin{tabular}{|c|c|}
\hline bit of aX-wind Landing \\
\hline Event \# (Downwind Check) \\
\hline MP & 71 (early) \\
\hline IAS & $70 \mathrm{kts}$ \\
\hline Flaps & Take-off \\
\hline Event \#(Base) \\
\hline \multicolumn{2}{|c|}{ Turn base } \\
\hline Event \# (Final) \\
\hline \multicolumn{2}{|c|}{ Turn final } \\
\hline \multicolumn{2}{|c|}{ Carb Heat ON } \\
\hline IAS (kts) & 60 \\
\hline Flaps & Landing \\
\hline Prop & 74 \\
\hline Event \# (Wheels Down) & 75 \\
\hline Brake to a stop as soon as possible without skidding tires \\
\hline Event \# (Stop)
\end{tabular}

$\frac{1}{4} x_{a^{k}} y^{a r}$ 


\section{B Flight Test Plan - Flight 2}

Pre-flight checks

\begin{tabular}{|c|c|}
\hline \multicolumn{2}{|c|}{ Take-off Holding Brakes } \\
\hline Wind Speed (kts) \\
\hline Wind Direction \\
\hline \multicolumn{2}{|c|}{ Pre-Take-off Check } \\
\hline \multicolumn{2}{|c|}{ Bring aircraft to a halt at start of runway } \\
\hline \multicolumn{2}{|c|}{ Apply full brakes } \\
\hline Apply full power and allow it to stabilize \\
\hline Event \# \\
\hline Engine (RPM) \\
\hline MP (in. Hg) & 25 \\
\hline Release brakes and use normal climb out \\
\hline Event \# (Wheels Up) & 2 \\
\hline Climb Speed (kts) & $(-0-65$ \\
\hline
\end{tabular}

Climb out, then transit to Practice Area

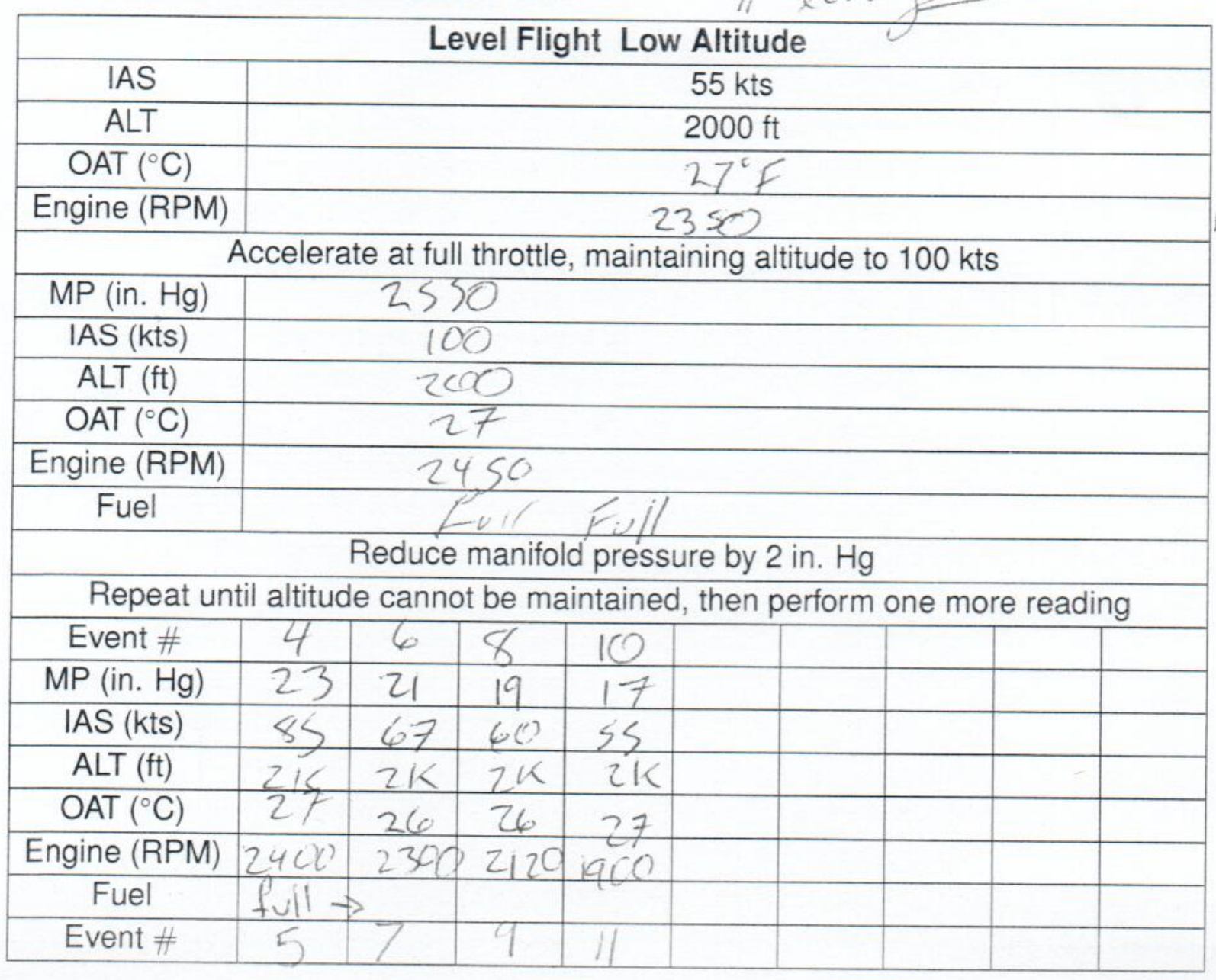




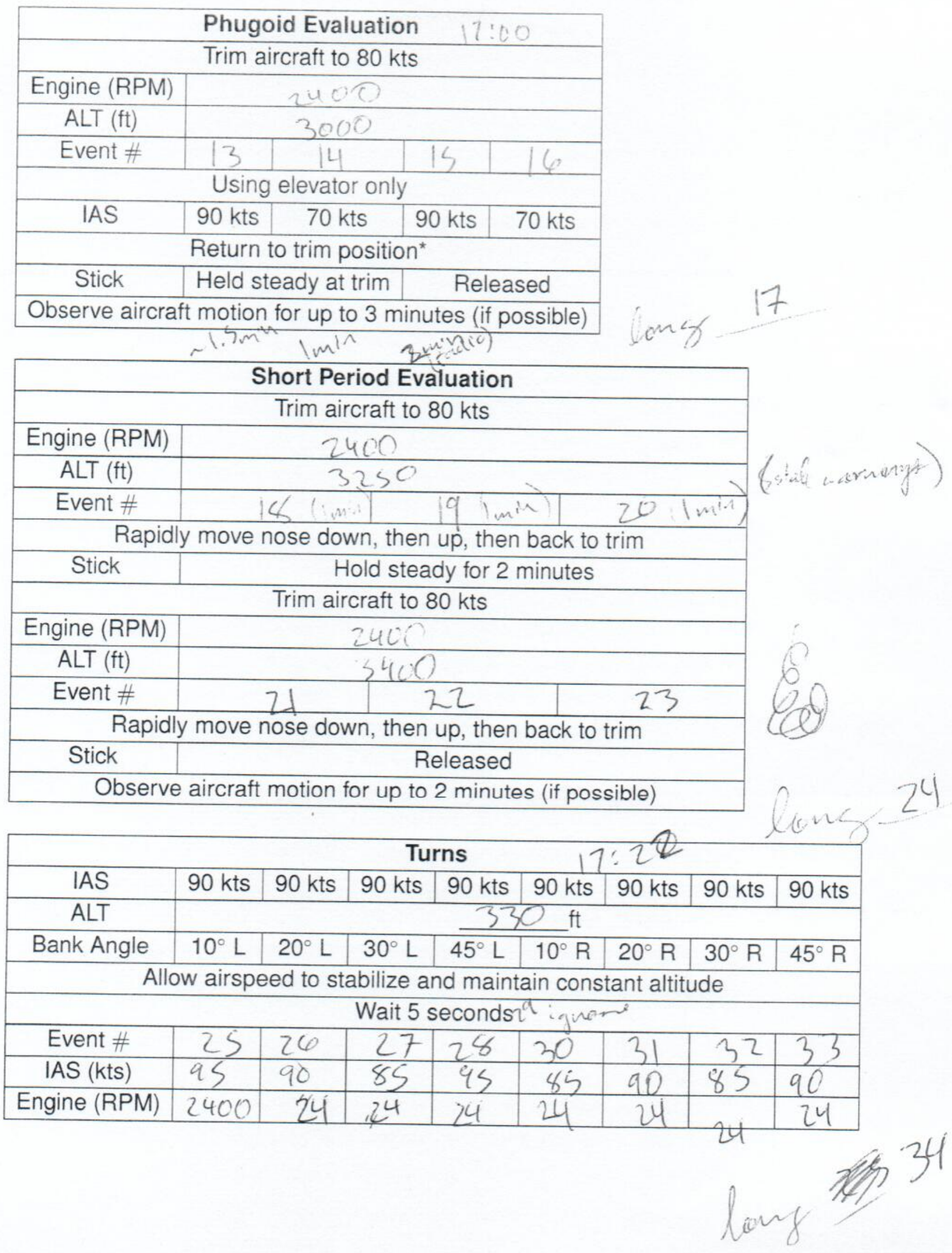




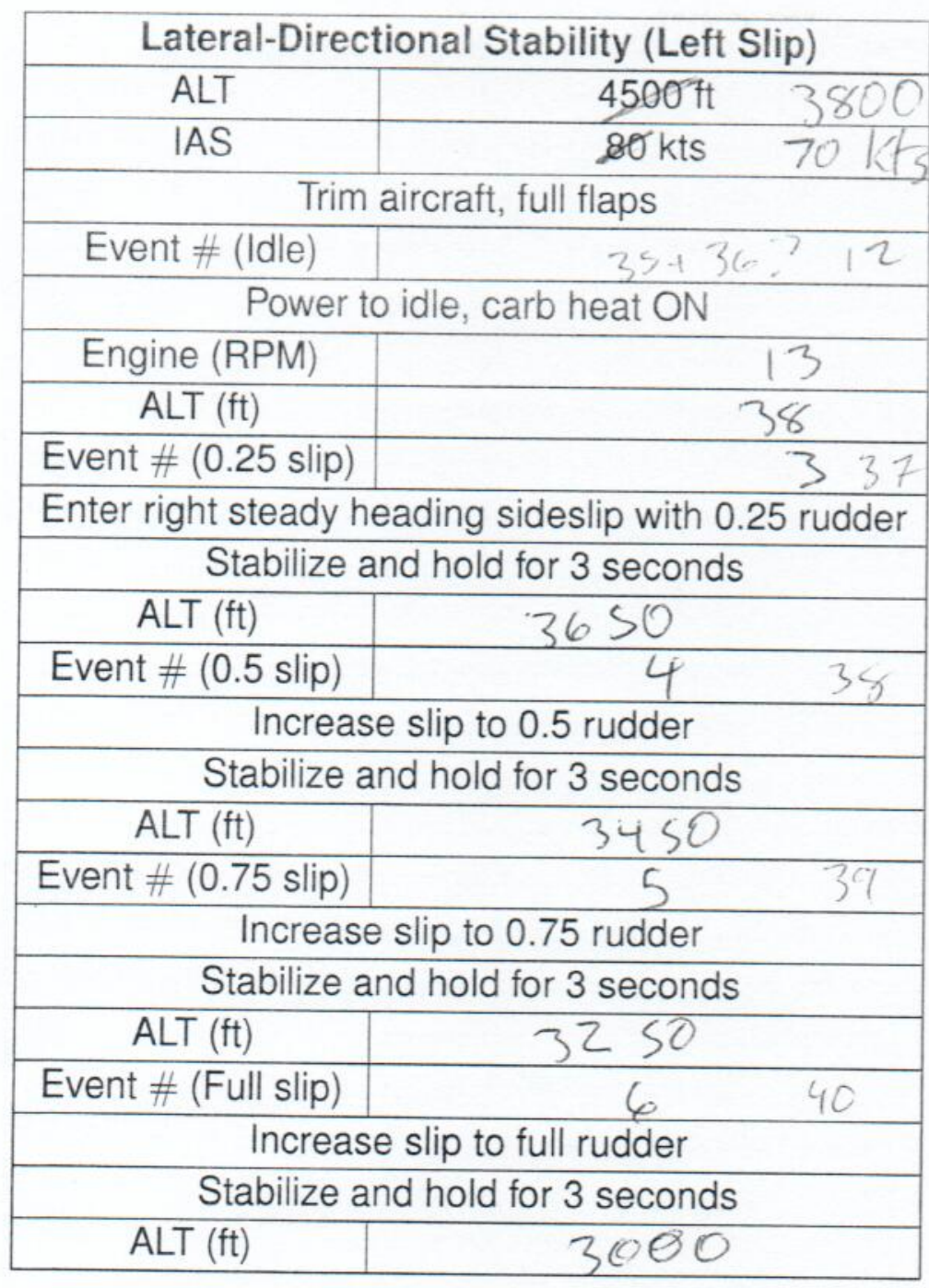

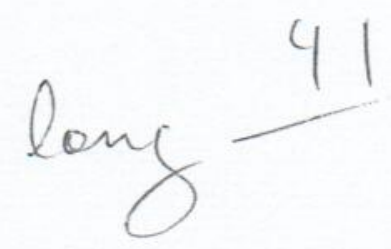




\begin{tabular}{|c|c|c|}
\hline \multicolumn{3}{|c|}{ Lateral-Directional Stability (Right Slip) } \\
\hline ALT & $4500 \mathrm{ft}$ & 4000 \\
\hline IAS & $-80 \mathrm{kts}$ & \\
\hline \multicolumn{3}{|c|}{ Trim aircraft, full flaps } \\
\hline Event \# (Idle) & 45 & \\
\hline \multicolumn{3}{|c|}{ Power to idle, carb heat ON } \\
\hline Engine (RPM) & 14 & \\
\hline $\mathrm{ALT}(\mathrm{ft})$ & 39 & \\
\hline Event \# (0.25 slip) & 46 & \\
\hline \multicolumn{3}{|c|}{ Enter right steady heading sideslip with 0.25 rudder } \\
\hline \multicolumn{3}{|c|}{ Stabilize and hold for 3 seconds } \\
\hline $\mathrm{ALT}(\mathrm{ft})$ & 3650 & \\
\hline Event \# (0.5 slip) & 47 & \\
\hline \multicolumn{3}{|c|}{ Increase slip to 0.5 rudder } \\
\hline \multicolumn{3}{|c|}{ Stabilize and hold for 3 seconds } \\
\hline $\mathrm{ALT}(\mathrm{ft})$ & 3450 & \\
\hline Event \# (0.75 slip) & 48 & \\
\hline \multicolumn{3}{|c|}{ Increase slip to 0.75 rudder } \\
\hline \multicolumn{3}{|c|}{ Stabilize and hold for 3 seconds } \\
\hline $\mathrm{ALT}(\mathrm{ft})$ & 3200 & \\
\hline Event \# (Full slip) & 49 & \\
\hline \multicolumn{3}{|c|}{ Increase slip to full rudder } \\
\hline \multicolumn{3}{|c|}{ Stabilize and hold for 3 seconds } \\
\hline $\operatorname{ALT}(\mathrm{ft})$ & 2950 & \\
\hline
\end{tabular}

Transit to YOW<smiles></smiles> 
B FLIGHT TEST PLAN - FLIGHT 2

24

\begin{tabular}{|c|c|}
\hline \multicolumn{2}{|c|}{ A Cl a Climb Evaluation } \\
\hline Heading & W \\
\hline \multicolumn{2}{|c|}{ Maintain heading (no flaps) } \\
\hline ALT (ft) & 95 \\
\hline IAS (ks) & $8 \mathrm{~K}$ \\
\hline Event \# (Climb) & $\$ 42$ \\
\hline \multicolumn{2}{|c|}{ Climb at $69 \mathrm{kts}$} \\
\hline \multicolumn{2}{|c|}{ Climb 2000 ft } \\
\hline Event \# (Level) \\
\hline \multicolumn{2}{|c|}{43} \\
\hline \multicolumn{2}{|c|}{ Level out } \\
\hline ALT (ft) & $4 / \mathrm{C}$ \\
\hline
\end{tabular}




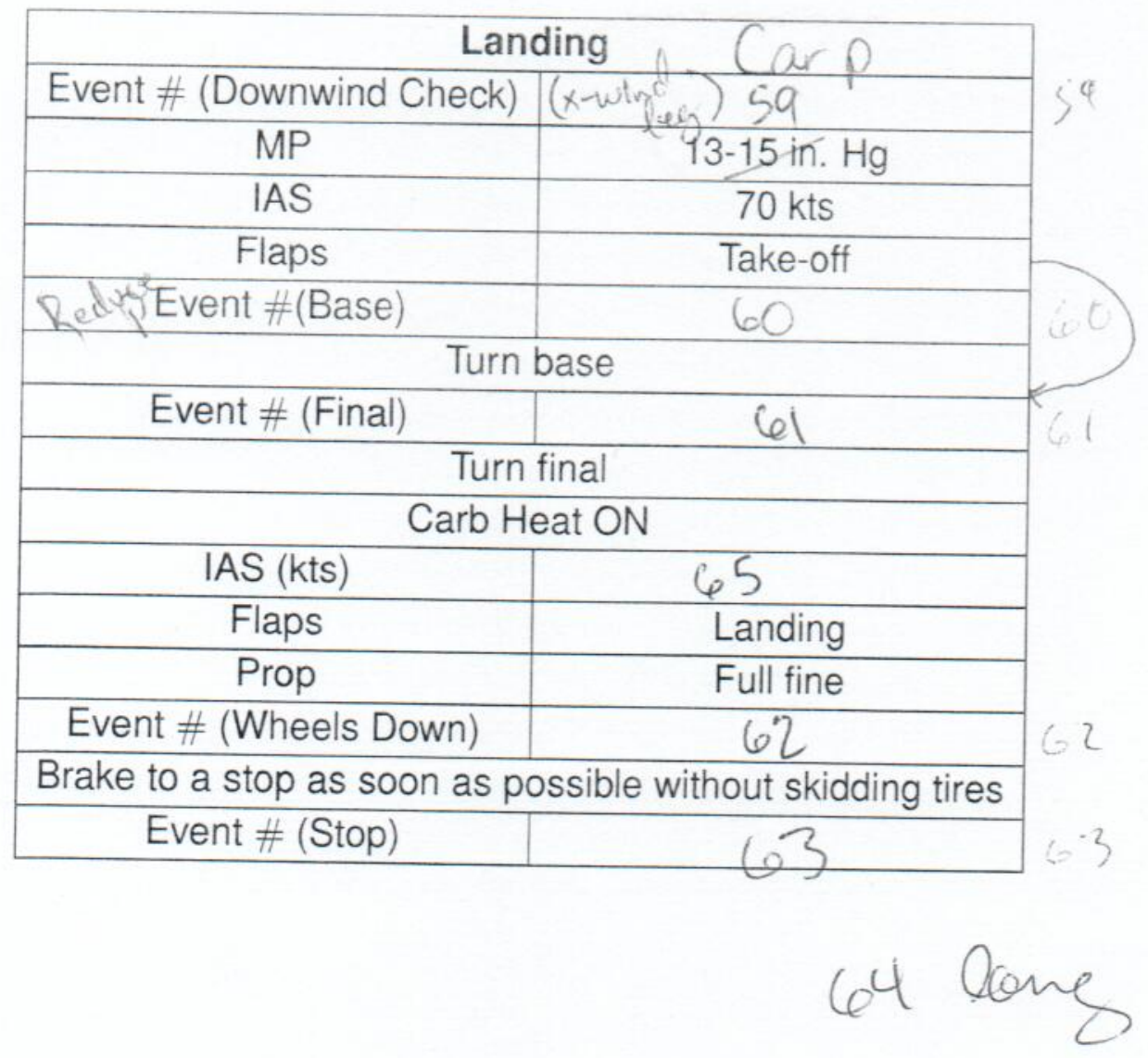




\section{Flight Test Plan - Flight 3}

Pre-flight checks

\begin{tabular}{|c|c|}
\hline \multicolumn{2}{|c|}{ Take-off Holding Brakes $\mathrm{C}$} \\
\hline Wind Speed (kts) & $310 @ 10$ \\
\hline Wind Direction & $29^{\circ}$ \\
\hline \multicolumn{2}{|c|}{ Pre-Takeoff Check } \\
\hline \multicolumn{2}{|c|}{ Bring aircraft to a halt at start of runway } \\
\hline \multicolumn{2}{|c|}{ Apply full brakes } \\
\hline \multicolumn{2}{|c|}{ Apply full power and allow it to stabilize } \\
\hline Event \# & 65 \\
\hline Engine (RPM) & 2450 \\
\hline MP (in. $\mathrm{Hg}$ ) & 29 \\
\hline \multicolumn{2}{|c|}{ Release brakes and use normal climb out } \\
\hline Event \# (Wheels Up) & 66 \\
\hline Climb Speed (kts) & 65 \\
\hline
\end{tabular}

Climb out, then transit to Practice Area

\begin{tabular}{|c|c|c|c|c|}
\hline \multicolumn{5}{|c|}{ Flight Path Stability } \\
\hline \multicolumn{5}{|c|}{ Propeller to Full Fine, No Flaps } \\
\hline \multicolumn{5}{|c|}{ Trim to $70 \mathrm{kts}$} \\
\hline ALT $(\mathrm{ft})$ & & & & \\
\hline \multicolumn{5}{|c|}{ Hold for 15 seconds } \\
\hline \multicolumn{5}{|c|}{ Vary airspeed using elevator only } \\
\hline \multicolumn{5}{|c|}{\begin{tabular}{|l|l|l|l} 
Event \# & & & \\
\end{tabular}} \\
\hline IAS & $65 \mathrm{kts}$ & $60 \mathrm{kts}$ & $75 \mathrm{kts}$ & $80 \mathrm{kts}$ \\
\hline $\operatorname{ALT}(\mathrm{ft})$ & 7 & & & \\
\hline \multicolumn{5}{|c|}{ Hold for 15 seconds } \\
\hline ALT $(\mathrm{ft})$ & & & & \\
\hline
\end{tabular}

\begin{tabular}{|c|c|c|c|c|}
\hline \multicolumn{5}{|c|}{ Phugoid Evaluation } \\
\hline \multicolumn{5}{|c|}{ Trim aircraft to $80 \mathrm{kts}$} \\
\hline \multicolumn{5}{|l|}{ Engine (RPM) } \\
\hline \multicolumn{5}{|l|}{$\mathrm{ALT}(\mathrm{ft})$} \\
\hline Event \# & & & $\ldots$ & \\
\hline \multicolumn{5}{|c|}{ Using elevator only } \\
\hline IAS & $90 \mathrm{kts}$ & $70 \mathrm{kts}$ & $90 \mathrm{kts}$ & $70 \mathrm{kts}$ \\
\hline \multicolumn{5}{|c|}{ Return to trim position* } \\
\hline Stick & \multicolumn{2}{|c|}{ Held steady at trim } & \multicolumn{2}{|c|}{ Released } \\
\hline Observe aircra & motion & r up to $3 \mathrm{~m}$ & nutes (if & ossible) \\
\hline
\end{tabular}


C FLIGHT TEST PLAN - FLIGHT 3

32

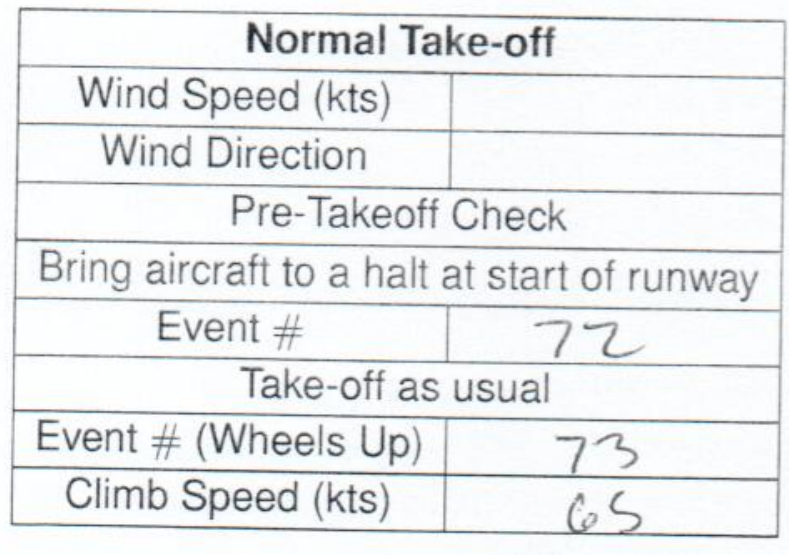

late by a second or 50 )

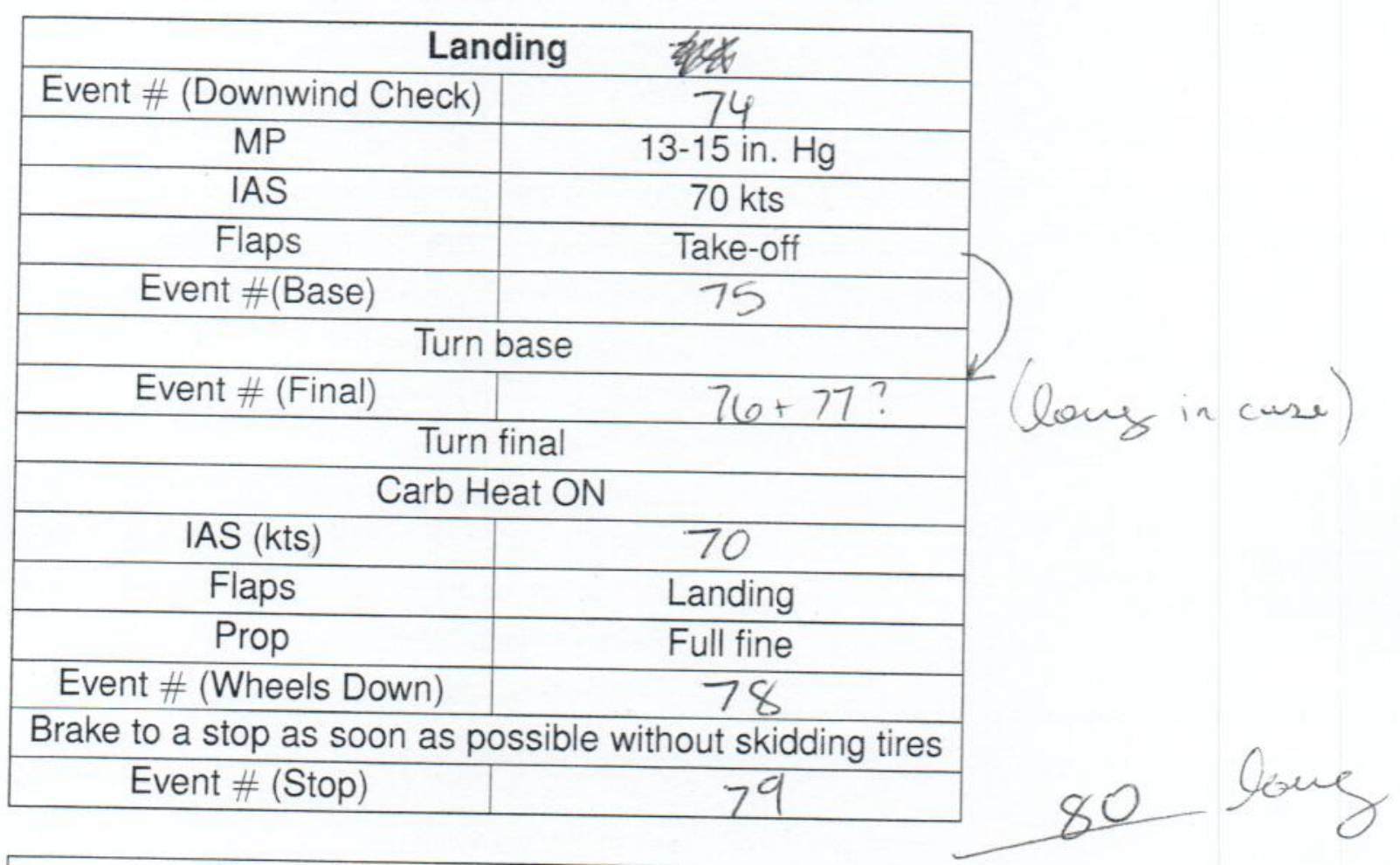

\begin{tabular}{|c|}
\hline \multicolumn{2}{|c|}{ Normal Take-off } \\
\hline Wind Speed (ks) \\
\hline Wind Direction \\
\hline \multicolumn{2}{|c|}{ Pre-Takeoff Check } \\
\hline Bring aircraft to a halt at start of runway \\
\hline \multicolumn{2}{|c|}{ Event \# } \\
\hline \multicolumn{2}{|c|}{ Take-off as usual } \\
\hline Event \# (Wheels Up) \\
\hline Climb Speed (ts) \\
\hline
\end{tabular}

82 long turn onto $x$. wind 
C FLIGHT TEST PLAN - FLIGHT 3

33

\begin{tabular}{|c|c|}
\hline \multicolumn{2}{|c|}{ Landing } \\
\hline Event \# (Downwind Check) & 83 \\
\hline MP & $13-15$ in. Hg \\
\hline IAS & $70 \mathrm{kts}$ \\
\hline Flaps & Takeoff \\
\hline Event \#(Base) & 84 \\
\hline \multicolumn{2}{|c|}{ Turn base } \\
\hline Event \# (Final) \\
\hline \multicolumn{2}{|c|}{ Turn final } \\
\hline \multicolumn{2}{|c|}{ Garb Heat ON } \\
\hline IAS (ks) & 65 \\
\hline Flaps & Landing \\
\hline Prop & Full fine \\
\hline Event \# (Wheels Down) & 66 \\
\hline Brake to a stop as soon as possible without skidding tires \\
\hline Event \# (Stop) & 67 \\
\hline
\end{tabular}

\begin{tabular}{|c|c|}
\hline \multicolumn{2}{|c|}{ Normal Take-off } \\
\hline Wind Speed (ks) & $300 @ 10$ \\
\hline Wind Direction \\
\hline \multicolumn{2}{|c|}{ Pre-Takeoff Check } \\
\hline Bring aircraft to a halt at start of runway \\
\hline \multicolumn{2}{|c|}{ Event \# } \\
\hline \multicolumn{2}{|c|}{69} \\
\hline Take-off as usual \\
\hline Event \# (Wheels Up) & 70 \\
\hline Climb Speed (ks) & $60-65$ \\
\hline
\end{tabular}

Transit to YOW

$18: 22$ 


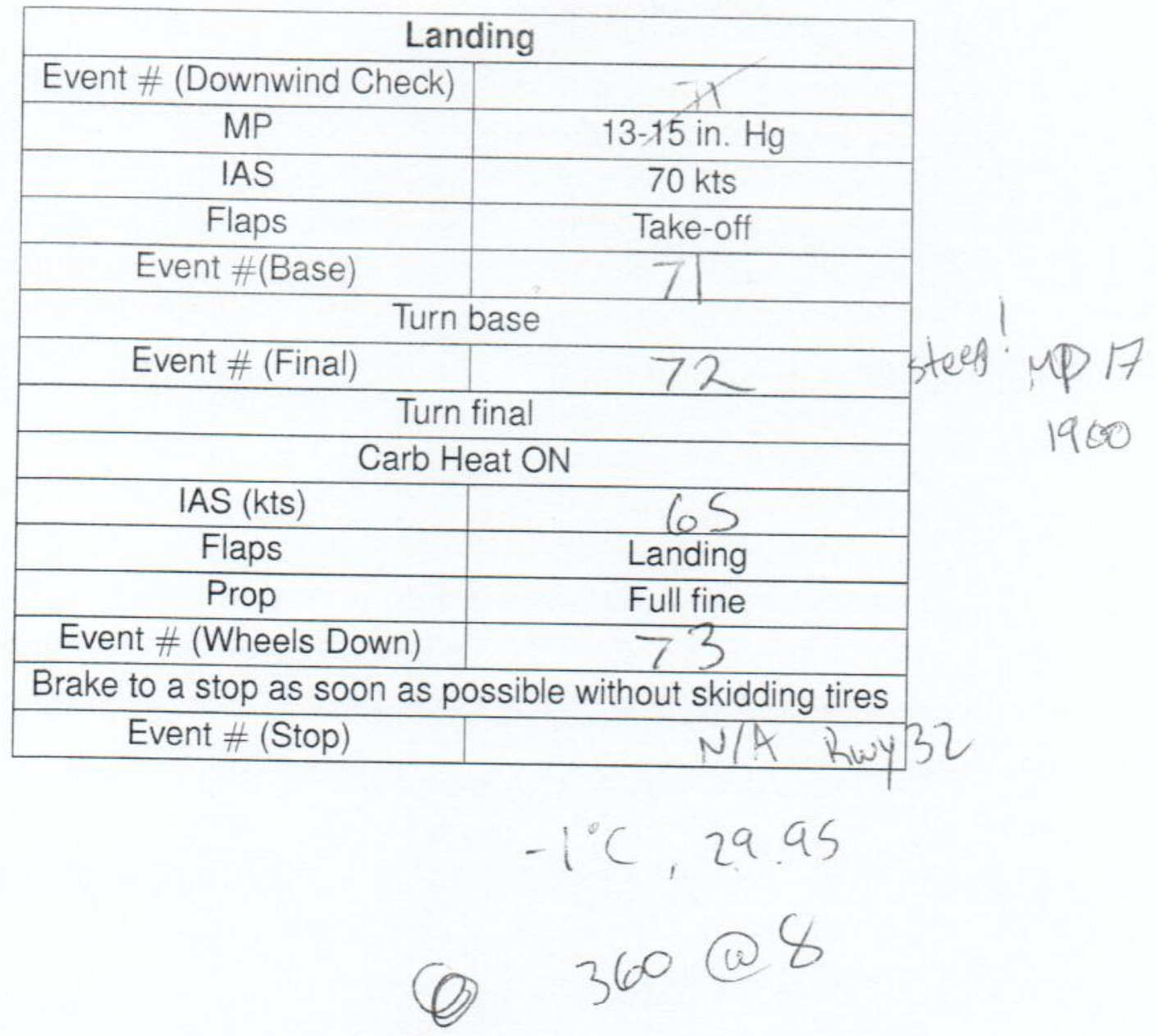




\section{Appendix E}

\section{Equations for Control Input Data Processing}

The expanded form of Equation 3.3 is

$$
\begin{aligned}
\vec{R}_{C / O}^{A C}= & {\left[\begin{array}{ccc}
1 & 0 & 0 \\
0 & \cos \theta & -\sin \theta \\
0 & \sin \theta & \cos \theta
\end{array}\right]\left[\begin{array}{l}
L_{A B_{I}} \\
L_{A B_{J}} \\
L_{A B_{K}}
\end{array}\right]+} \\
& {\left[\begin{array}{ccc}
1 & 0 & 0 \\
0 & \cos \theta & -\sin \theta \\
0 & \sin \theta & \cos \theta
\end{array}\right]\left[\begin{array}{ccc}
\cos \beta & 0 & \sin \beta \\
0 & 1 & 0 \\
\sin \beta & 0 & \cos \beta
\end{array}\right]\left[\begin{array}{l}
L_{B C_{i}} \\
L_{B C_{j}} \\
L_{B C_{k}}
\end{array}\right]+\left[\begin{array}{l}
L_{A O_{x}} \\
L_{A O_{y}} \\
L_{A O_{z}}
\end{array}\right] }
\end{aligned}
$$

where it is known that

$$
L_{A B_{I}}=L_{A B_{J}}=L_{B C_{i}}=0
$$


The expanded form of Equation 3.8 is

$$
\begin{aligned}
f(\theta)= & r_{O}^{2}-x_{i n t}^{2}-L_{A O_{y}}^{2}-L_{A O_{z}}^{2}-L_{A B_{K}}^{2}-L_{B C_{j}}^{2}-L_{B C_{k}}^{2} \cos ^{2} \beta+ \\
& 2 L_{A O_{y}} L_{A B_{K}} \sin \theta-2 L_{A O_{z}} L_{A B_{K}} \cos \theta-2 L_{A O_{y}} L_{B C_{j}} \cos \theta- \\
& 2 L_{A O_{z}} L_{B C_{j}} \sin \theta+2 L_{A O_{y}} L_{B C_{k}} \cos \beta \sin \theta-2 L_{A O_{z}} L_{B C_{k}} \cos \beta \cos \theta \\
& -2 L_{A B_{K}} L_{B C_{k}} \cos \beta
\end{aligned}
$$

and its derivative, required for the Newton-Raphson method of solving for the equation roots, is

$$
\begin{aligned}
f^{\prime}(\theta)= & 2 L_{A O_{y}} L_{A B_{K}} \cos \theta+2 L_{A O_{z}} L_{A B_{K}} \sin \theta+2 L_{A O_{y}} L_{B C_{j}} \sin \theta \\
& -2 L_{A O_{z}} L_{B C_{j}} \cos \theta+2 L_{A O_{y}} L_{B C_{k}} \cos \beta \cos \theta+2 L_{A O_{z}} L_{B C_{k}} \cos \beta \sin \theta
\end{aligned}
$$


Appendix F

Sensitivity Study Results 


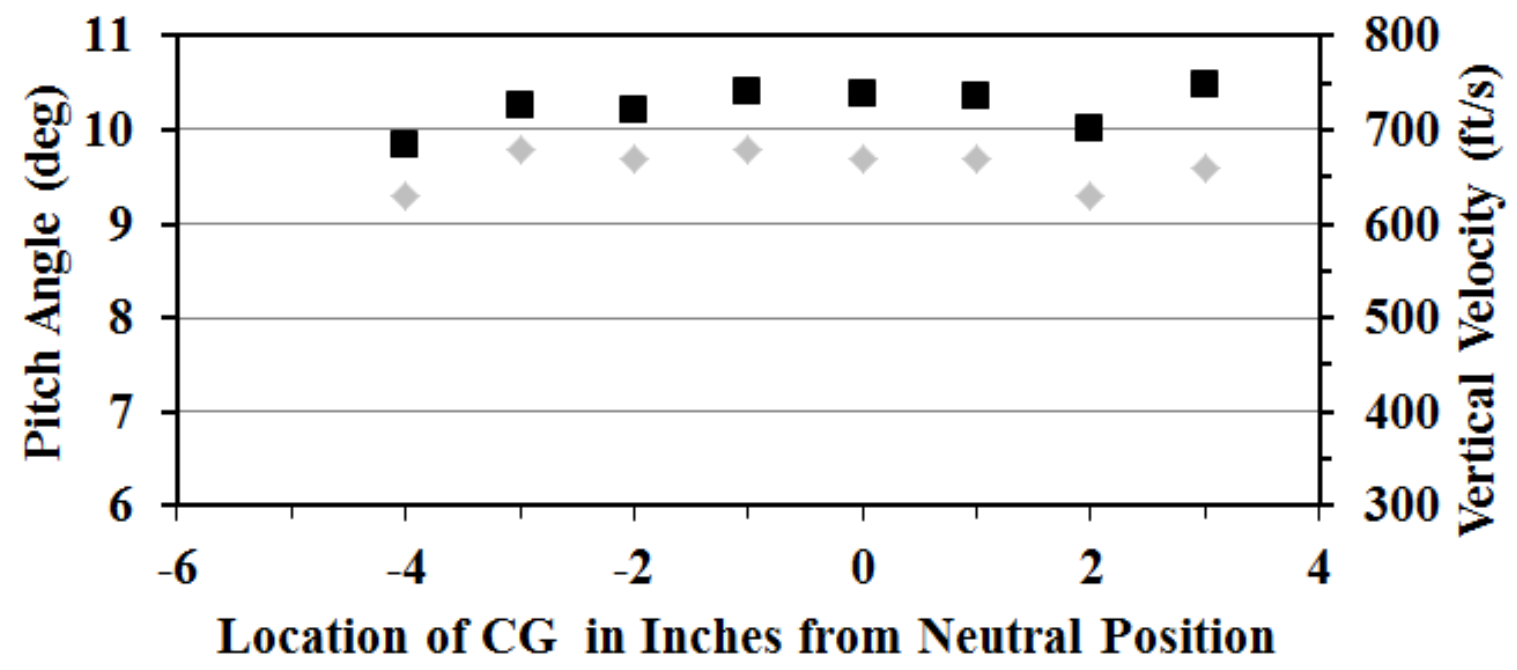

Figure F.1: Effect of CG location on take-off climb performance, specifically the pitch angle (gray) and climb rate (black).

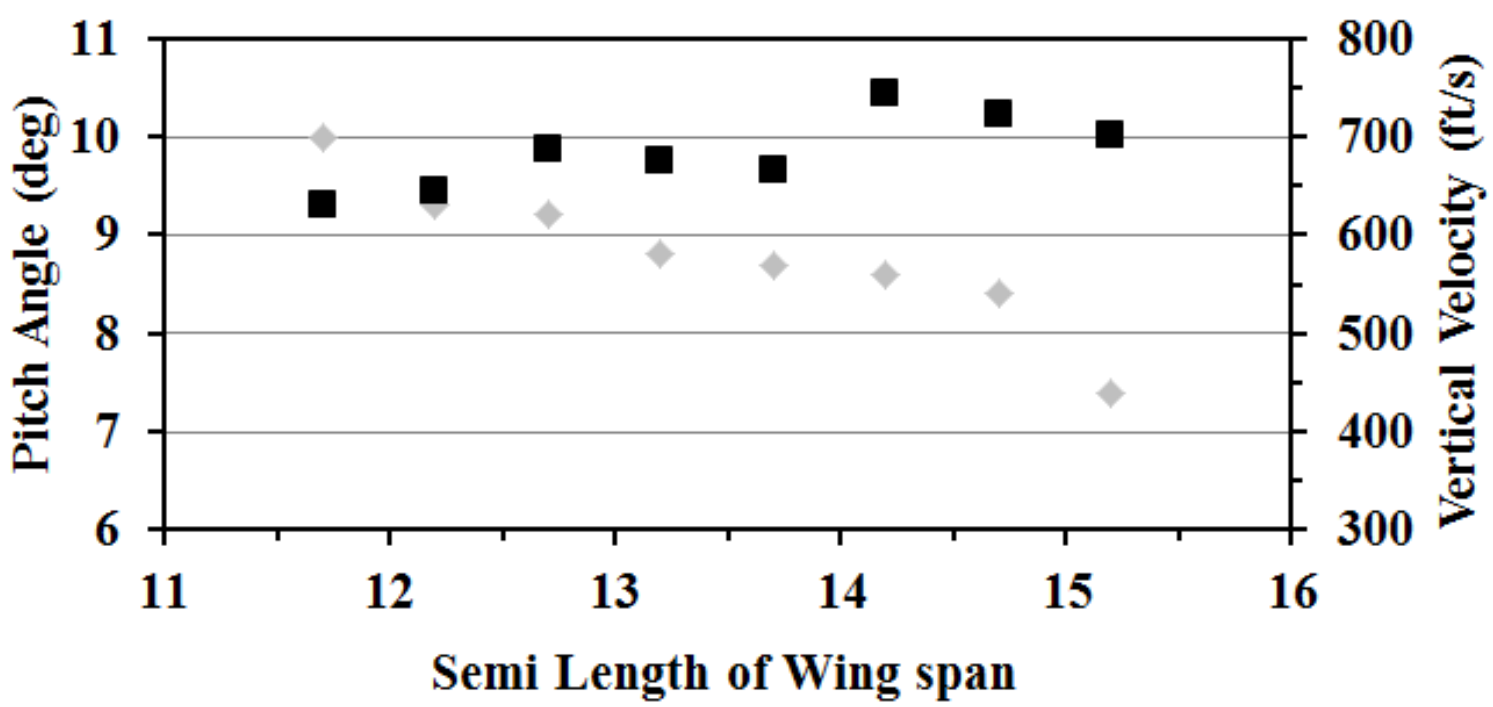

Figure F.2: Effect of wing span on take-off climb performance, specifically the pitch angle (gray) and climb rate (black). 


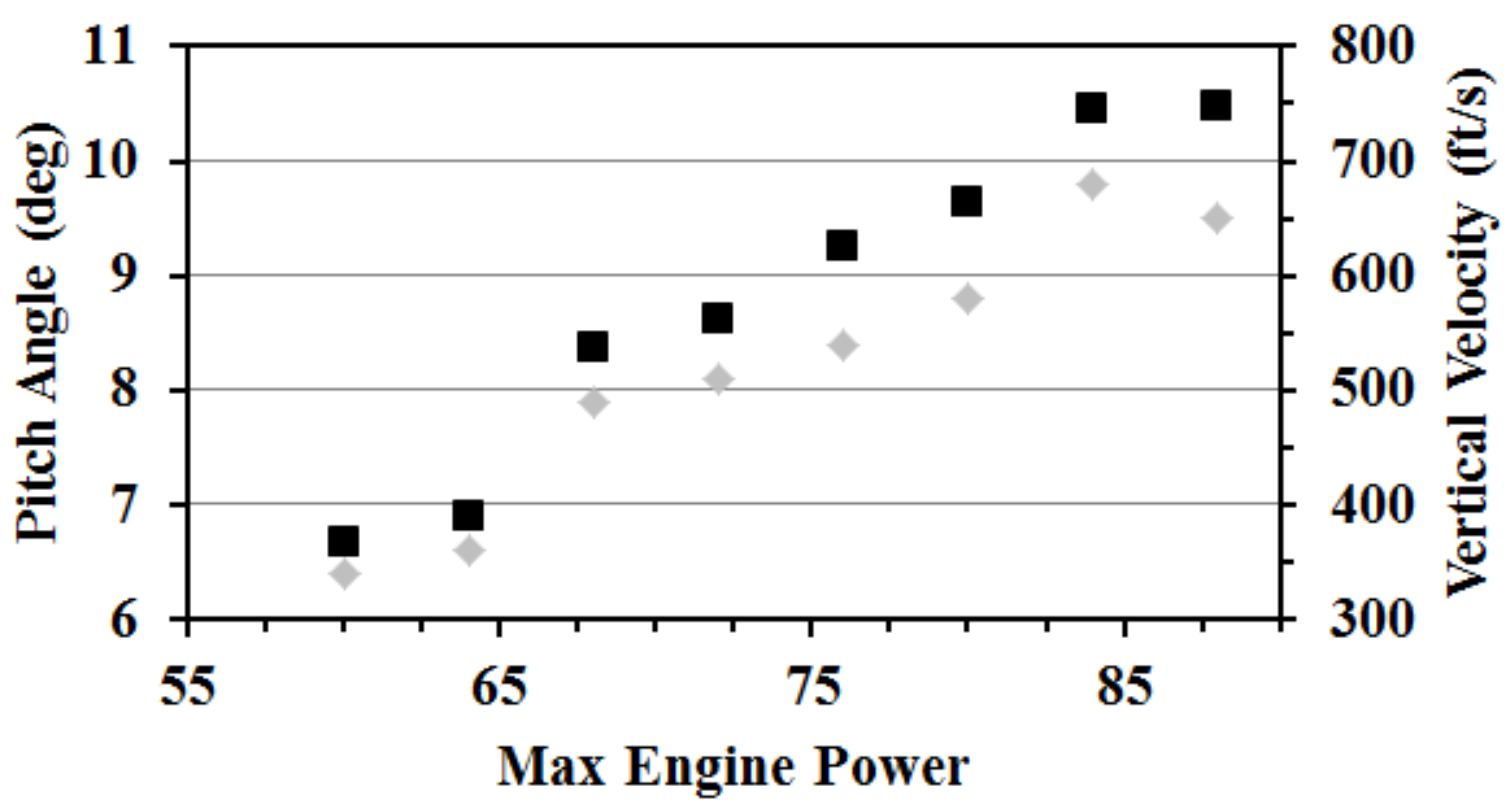

Figure F.3: Effect of maximum available engine power on take-off climb flight performance, specifically the pitch angle (gray) and climb rate (black).

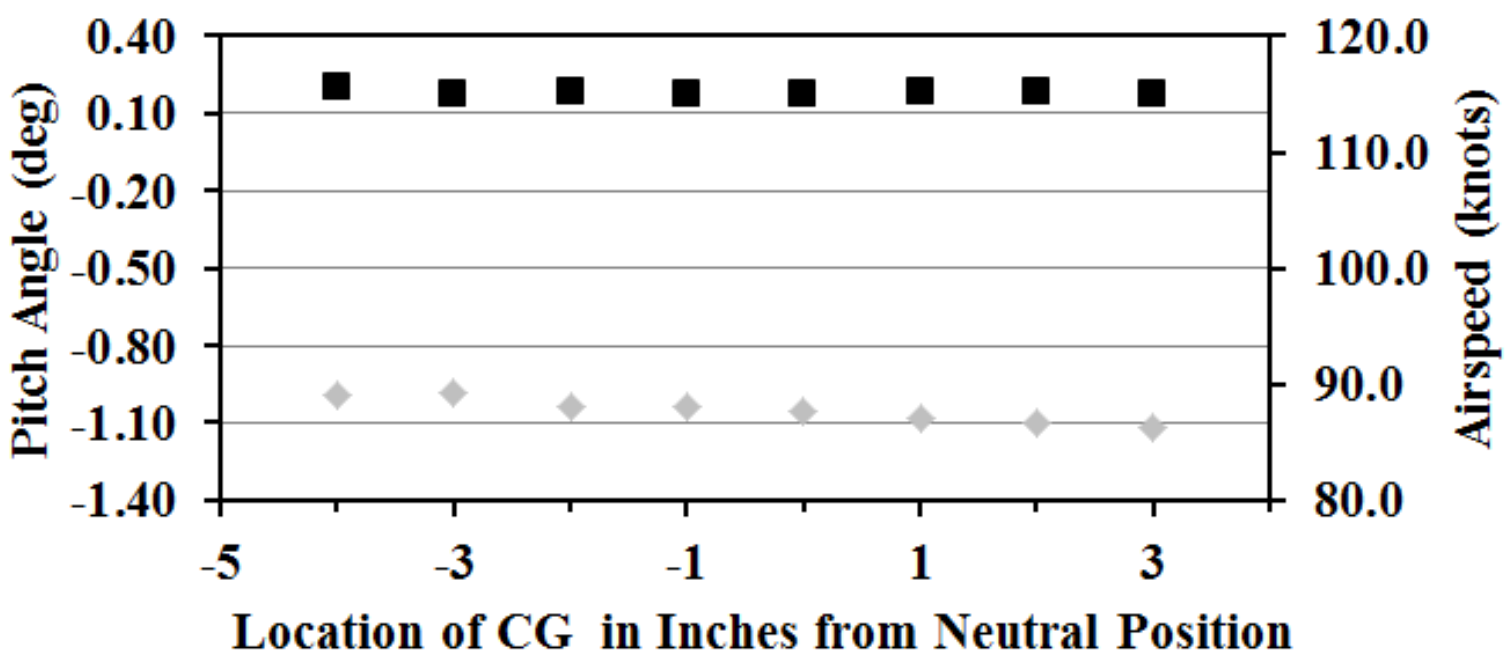

Figure F.4: Effect of CG location on straight and level flight performance, specifically the pitch angle (gray) and cruise speed (black). 


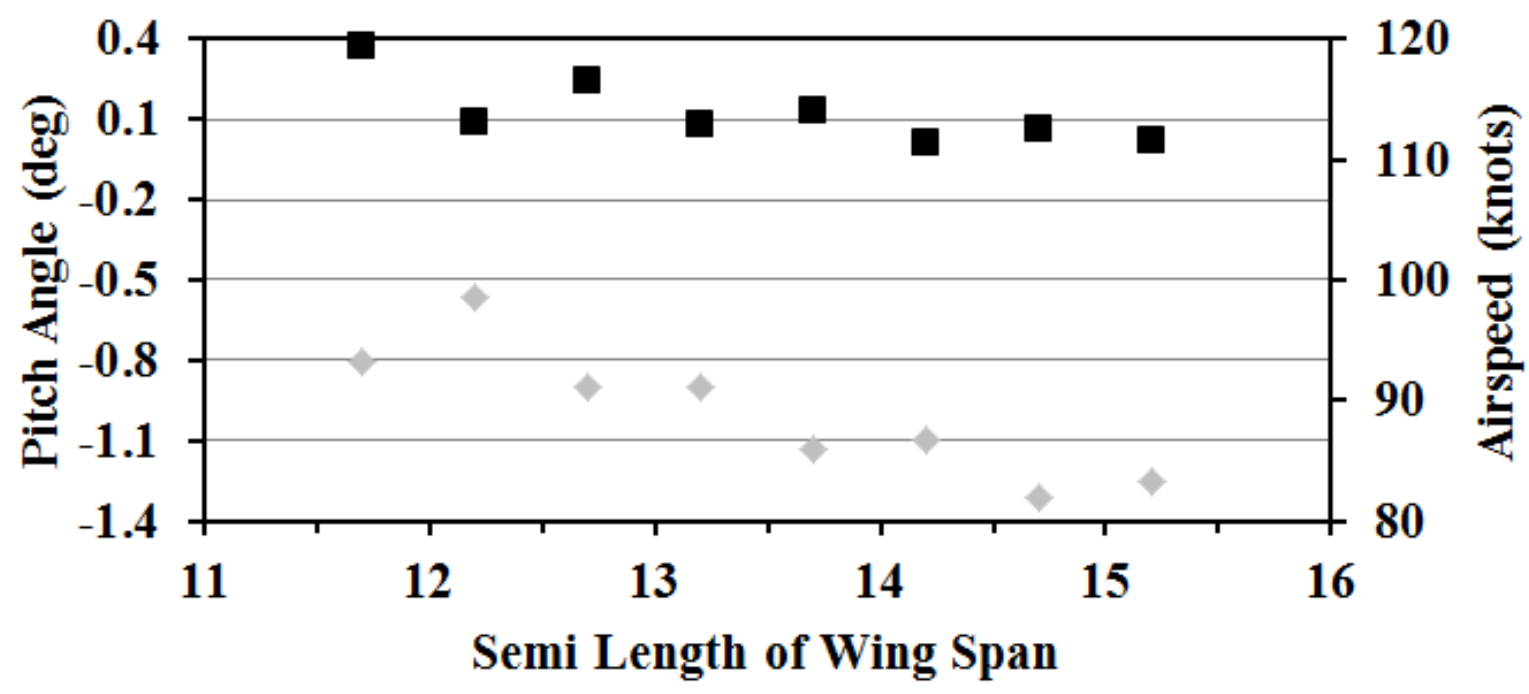

Figure F.5: Effect of wing span on straight and level flight performance, specifically the pitch angle (gray) and cruise speed (black).

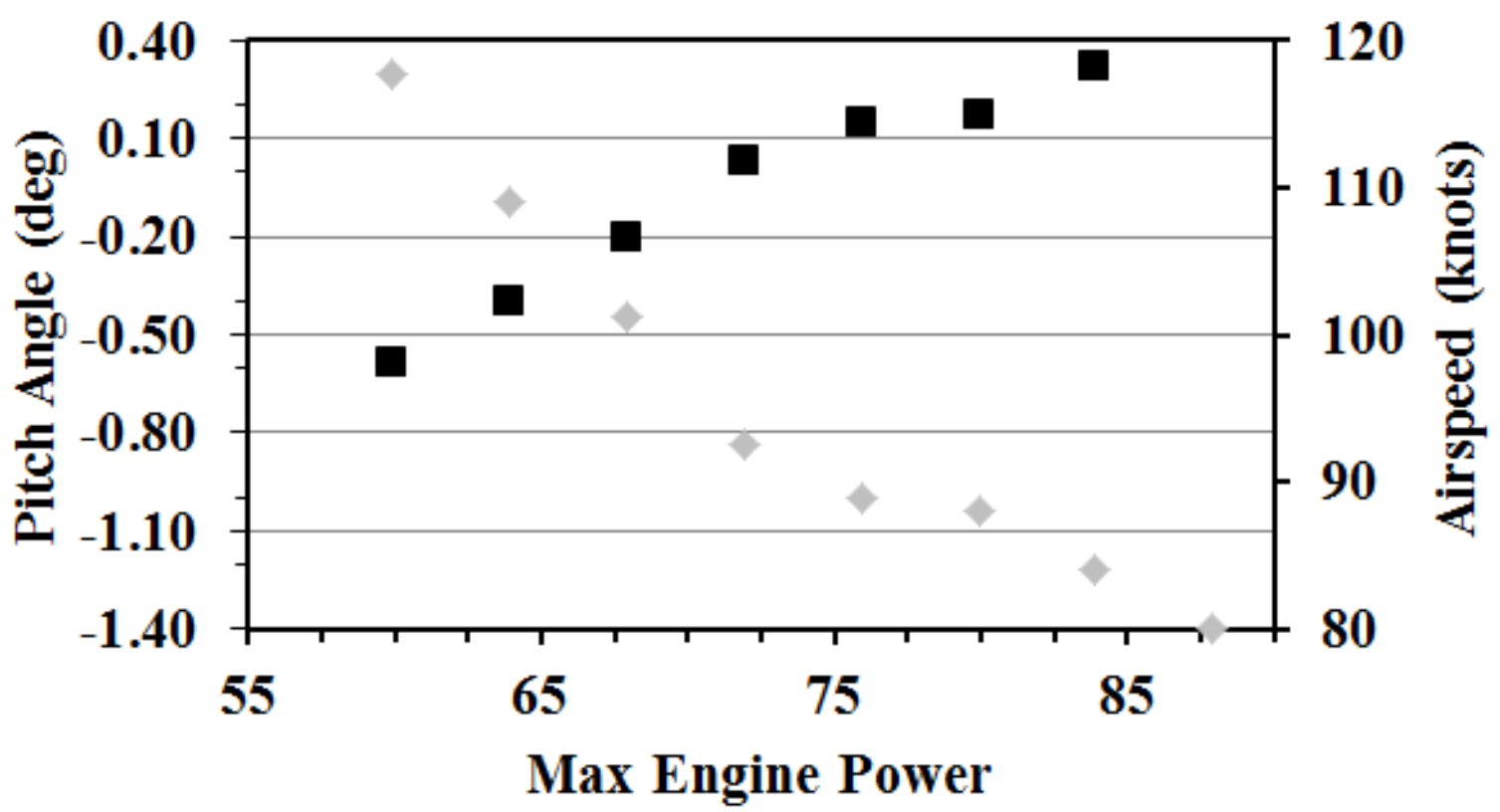

Figure F.6: Effect of maximum available engine power on straight and level flight performance, specifically the pitch angle (gray) and cruise speed (black). 